\title{
The Molecular Bases of Training Adaptation
}

A thesis submitted in fulfilment of the requirements for the Doctorate of Philosophy

\section{Vernon G. Coffey}

School of Medical Sciences

Science, Engineering and Technology Portfolio

RMIT University

August 2006 


\section{Publications arising from this thesis}

This thesis is based on the following publications:

1. Coffey, V.G., Reeder, D.W., Lancaster, G.I., Yeo, W.K., Febbraio, M.A., Yaspelkis III, B.B., Hawley, J.A. (2006). High-frequency training stimulus attenuates AKT and exacerbates $\mathrm{TNF} \alpha$ signaling responses in skeletal muscle. Journal of Applied Physiology (In Review).

2. Coffey V.G., Zhong Z., Shield A., Canny B.J., Chibalin A.V., Zierath J.R, Hawley J.A. (2006). Early signaling responses to divergent exercise stimuli in skeletal muscle from well-trained humans. The FASEB Journal 20(1): 190-2.

3. Coffey V.G., Shield A., Canny B.J., Carey K.A., Cameron-Smith D., Hawley J.A. (2006). Interaction of contractile activity and training history on mRNA abundance in skeletal muscle from trained humans. American Journal of Physiology Endocrinology and Metabolism 290(5): E849-855.

Additional publications arising from work undertaken for this thesis:

4. Deshmukh A., Coffey V.G., Zhong Z., Chibalin A.V., Zierath J.R, Hawley J.A. (2006). Exercise-induced phosphorylation of the novel Akt substrates AS160 and filamin in human skeletal muscle. Diabetes 55(6): 1776-1782.

5. Coffey V.G. and Hawley, J.A. (2006). Training for performance: Insights from molecular biology. Invited commentary. International Journal of Sports Physiology and Performance 1: 347-355.

6. Coffey, V.G. \& Hawley, J.A. (2005). The Specificity of Training: New Insights from Molecular Biology. Invited Review for Inaugural Issue. Malaysian Journal of Sport Science and Recreation 1 (1): 1-15. 


\section{Declaration}

I certify that except where due acknowledgement has been made, the work is that of the author alone; the work has not been submitted previously, in whole or in part, to qualify for any other academic award; the content of the thesis is the result of work which has been carried out since the official commencement date of the approved research programme; and, any editorial work, paid or unpaid, carried out by a third party is acknowledged.

Signed:

Vernon G. Coffey

Date: 


\section{Acknowledgements}

First, and foremost, I would like to acknowledge and thank my principle supervisor John Hawley for giving me the opportunity to study and learn from a world renowned exercise physiologist, and for guidance and support throughout my candidature. It is rare for a student to be allowed and equipped to express and develop research ideas and have such a substantial input into doctoral study, for which I am particularly grateful. I would also like to thank Anthony Shield for his help and input into this thesis, and for always being willing to provide valuable insight with regard to the projects outlined in this work.

Numerous individuals contributed to the studies incorporated in this thesis:

- Thank you to Professor Juleen Zierath at the Karolinska Institute, Stockholm, Sweden and her lab members Alexander Chibalin and Zhihui Zong for the protein analysis for my second study.

- Thank you to Dr. Ben Canny M.D. for performing all the muscle biopsies during human exercise trials.

- Thank you to Dr. David Cameron-Smith and lab members Dr. Kate Carey and Janelle Mollica from Deakin University, Melbourne for technical assistance during mRNA analysis.

- Thanks also to Dr. Ben Yaspelkis III and particularly Don Reeder from California State University, Northridge, U.S.A. who spent countless hours to facilitate the exercise model for the rodent resistance training study.

- I would also like to thank Dr. Graeme Lancaster for his help providing IKK analysis, and Wee Kian Yeo and Emmanuel Churchley for muscle glycogen data. 
I would want to say thanks to all the past and present members of the Exercise Metabolism Group who have been the principle reason for the enjoyment I have had during my $3 \frac{1}{2}$ years at RMIT University. The value of this experience is found in the people I have shared it with.

I would especially like to thank Sarah Lessard, with whom I have shared my entire $\mathrm{PhD}$ journey, for her friendship and help during this rollercoaster ride. Unfortunately, as time goes by our professional lives will probably take us down different paths but I hope our friendship will last a life time. Maybe we can, at the very least, catch up at EMG lab reunions when John is old and grey!

Thank you to my parents, family and friends who never seem to doubt me and support me in everything I do.

Special thanks to my wife Heather who believes in me more than I believe in myself, rest assured my heart is all yours.

Finally, should this thesis represent achievement, bring recognition or commendation of any kind, I would acknowledge my friend and Saviour Jesus Christ who makes me who I am and with whose gifts I have done my best.

"Wisdom may satisfy the needs of the mind but it will never satisfy the needs of the soul."

So... set your mind on things above, not earthly things... and whatever you do, whether in word or deed, do it all in the name of the Lord Jesus, giving thanks to the Father through Him Col 3:2, 17 . 


\section{Table of Contents}

PUBLICATIONS ARISING FROM THIS THESIS ......................................................................

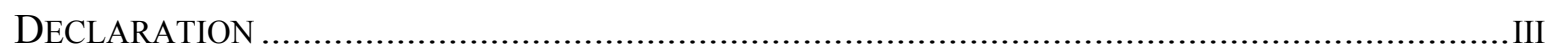

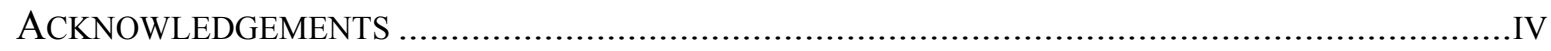

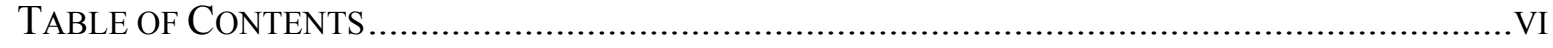

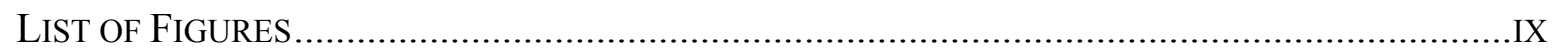

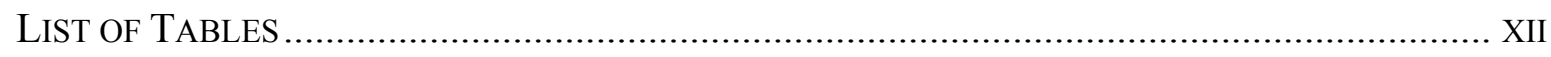

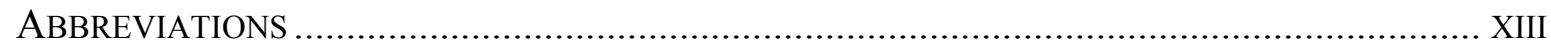

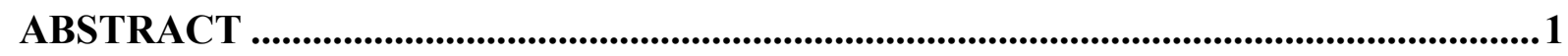

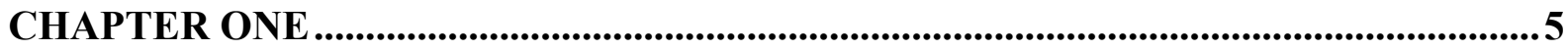

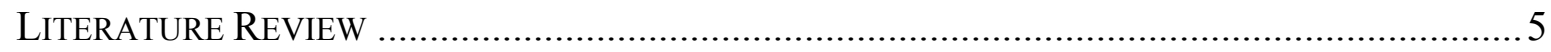

1.1 Overview: The Specificity of Exercise Responses .................................................... 6

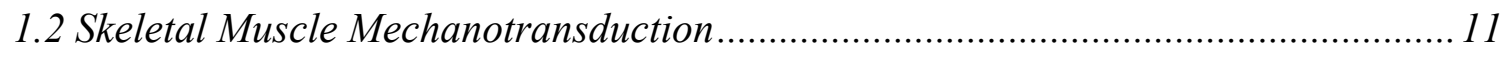

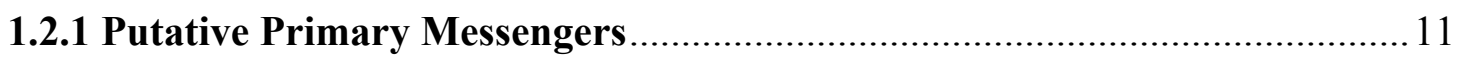

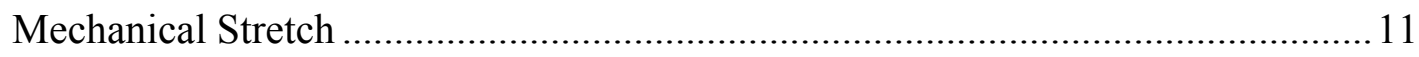

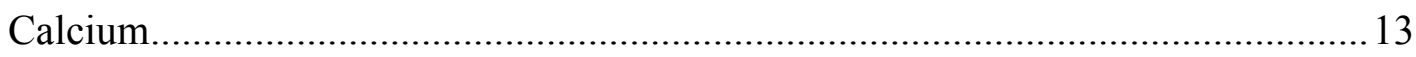

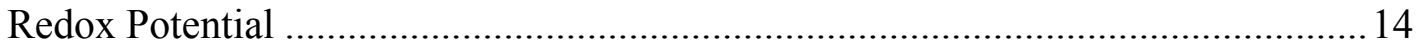

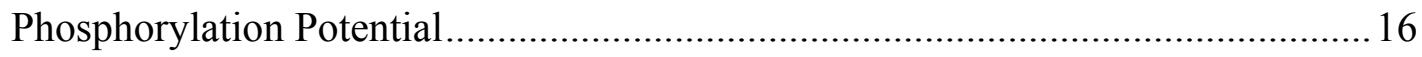

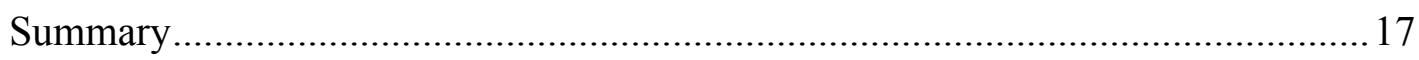

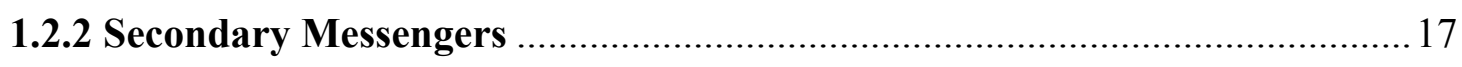

5'Adenosine Monophosphate Activated Protein Kinase (AMPK) Mediated

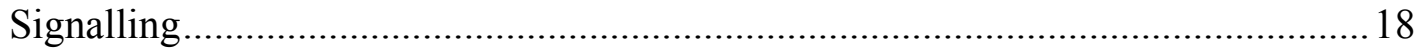

$\mathrm{Ca}^{2+}$ calmodulin-dependent kinase (CaMK) / Calcineurin Signalling .....................22

Insulin / Insulin-like Growth Factor (IGF) Signalling Pathway............................25

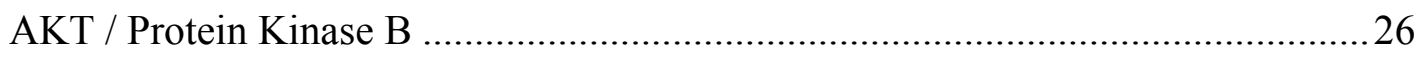




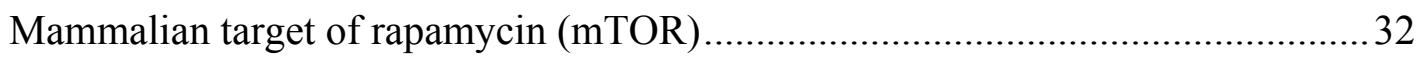

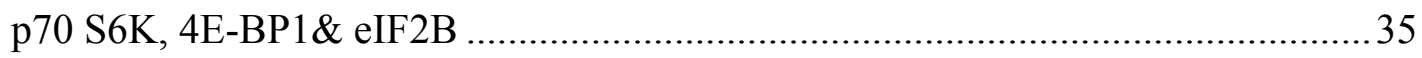

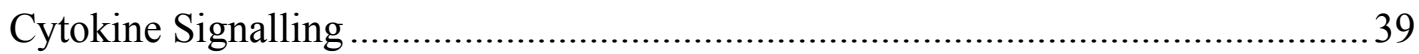

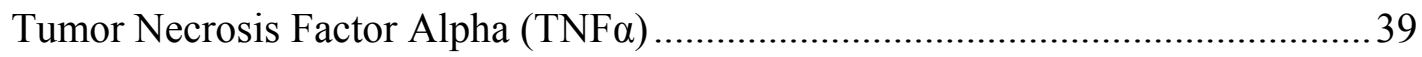

Inhibitor Kappa B Kinase (IKK) \& Nuclear Factor Kappa B (NFкB)................... 41

Mitogen Activated Protein Kinase (MAPK) Signalling Pathway ............................44

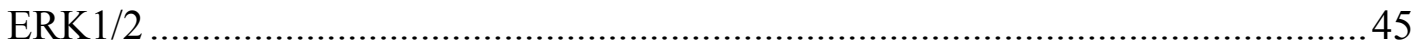

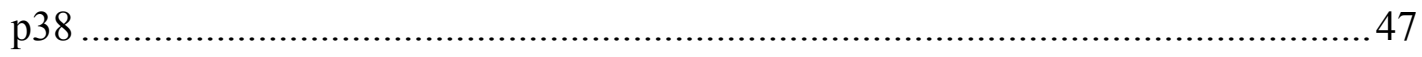

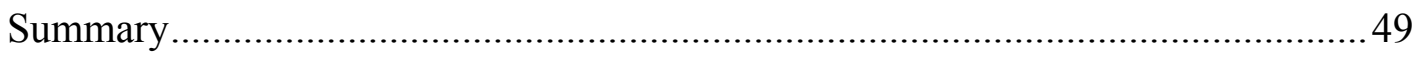

1.3 Genetic and Molecular Responses to Exercise........................................................ 49

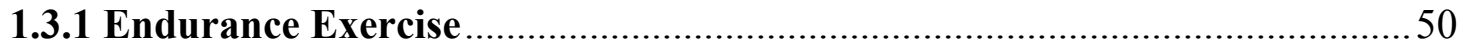

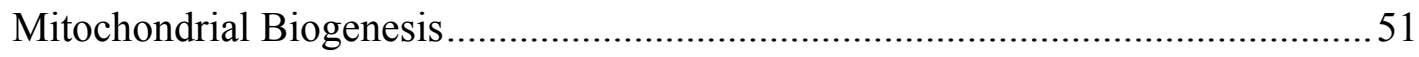

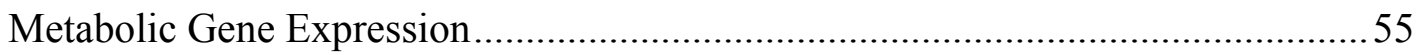

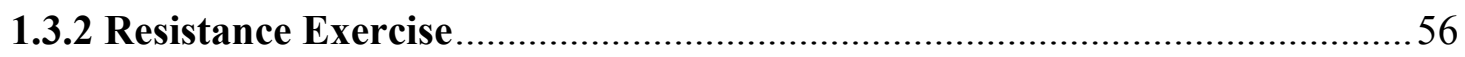

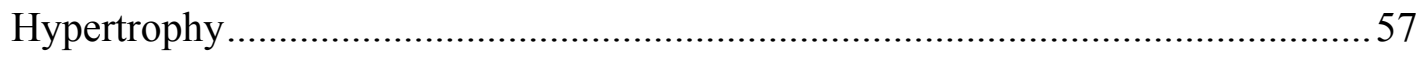

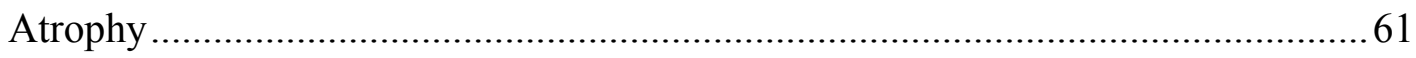

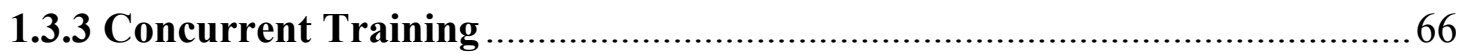

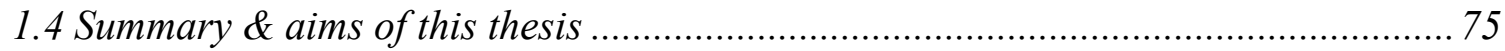

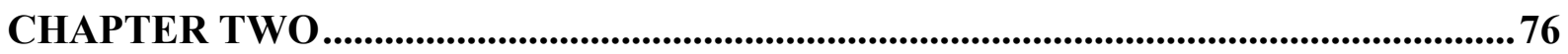

HIGH-FREQUENCY TRAINING STIMULUS ATTENUATES AKT AND EXACERBATES TNFA

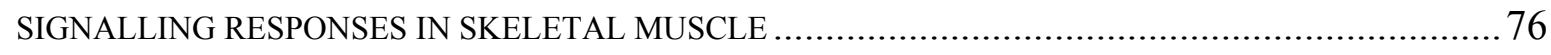

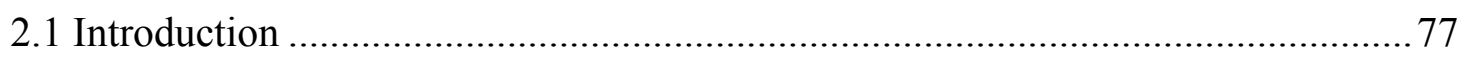

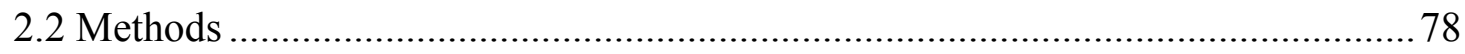

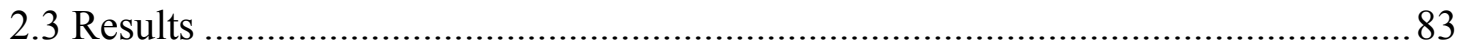

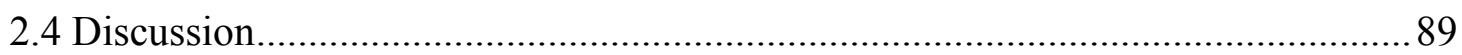


EARLY SIGNALLING RESPONSES TO DIVERGENT EXERCISE STIMULI IN SKELETAL MUSCLE

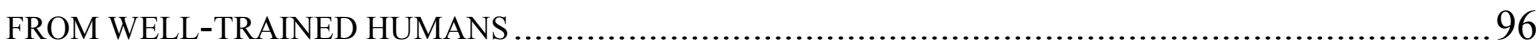

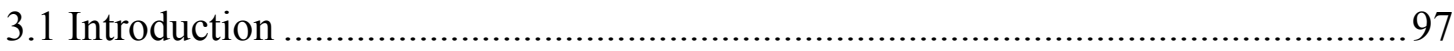

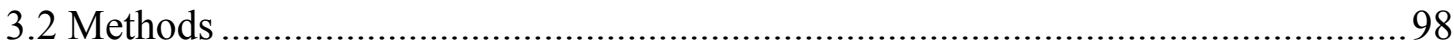

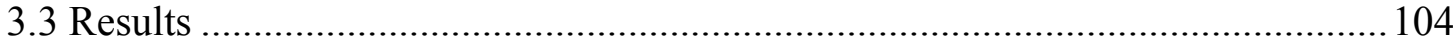

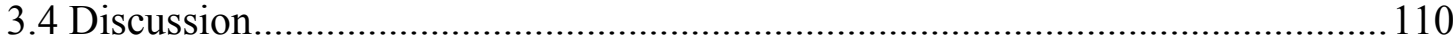

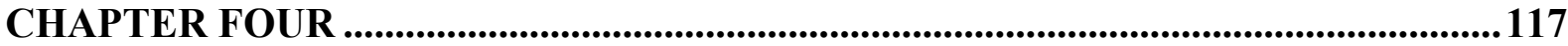

INTERACTION OF CONTRACTILE ACTIVITY AND TRAINING HISTORY ON MRNA ABUNDANCE IN

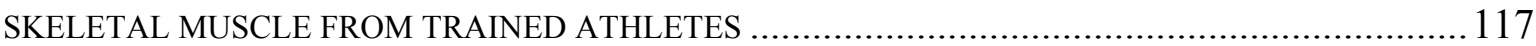

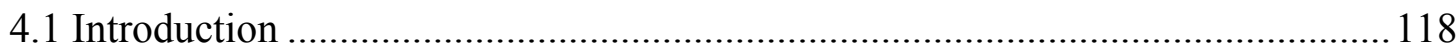

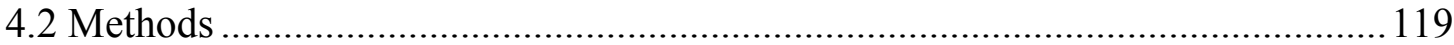

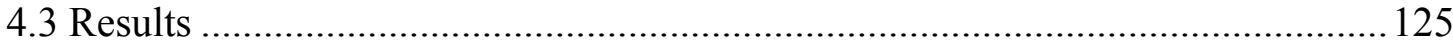

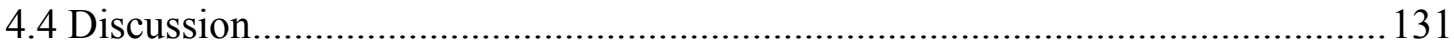

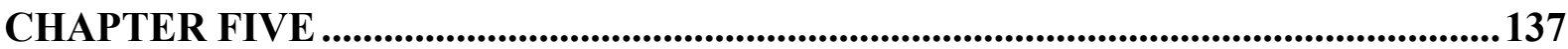

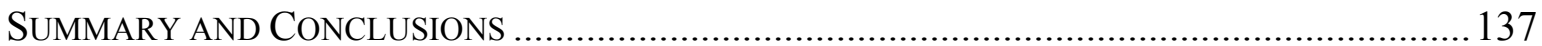

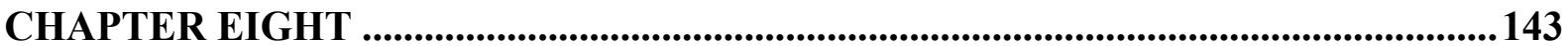

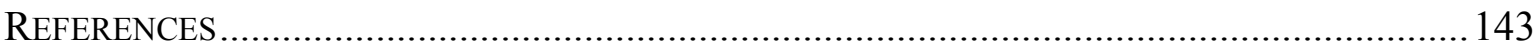




\section{List of Figures}

Figure 1.1 Putative steps through which contractile activity leads to alterations of skeletal muscle

Figure 1.2 Schematic of the putative (A) mRNA and (B) protein responses with adaptation to repetitive exercise stress. 8

Figure 1.3 Proposed effect of concurrent training on specific adaptation when compared to single mode training.

Figure 1.4 Metabolic and mechanical signals believed to play key roles as primary messengers in mechanotransduction. 14

Figure 1.5 Putative contraction-mediated adaptive events associated with 5'Adenosine Monophosphate Activated Protein Kinase (AMPK) activation in skeletal muscle.

Figure 1.6 Proposed mechanisms for calcium-dependent increases in gene expression with skeletal muscle contractile activity.

Figure 1.7 Simplified IGF-1 signalling pathway from receptor binding to protein synthesis. 26

Figure 1.8 Proposed adaptive events associated with activation of AKT in skeletal muscle..27

Figure 1.9 Model of the regulation and functions of the mammalian target of rapamycin (mTOR) complex.

Figure 1.10 Putative downstream targets and functions of ribosomal protein S6K.

Figure 1.11 Simplified TNF $\alpha$ pathway initiating nuclear transcription.

Figure 1.12 Proposed pathways of activation and molecular targets of MAPK signalling.....44

Figure 1.13 Five stages of gene expression and sites of regulation..... 50

Figure 1.14 Peroxisome proliferator receptor delta (PPAR $\delta)$ regulates exercise endurance. .54

Figure 1.15 Schematic representations of events regulating satellite cell activation. 59

Figure 1.16 Putative pathways regulating signalling to MAFBx / MuRF ubiquitin ligases. ..63 
Figure 1.17 Schematic providing an example of the integration of hypertrophy (IGF-1) and atrophy $(\mathrm{TNF} \alpha)$ pathways in skeletal muscle. 66

Figure 1.18 Putative divergent regulation of eukaryotic elongation factor 2 .....

Figure 1.19 Proposed effects of acute endurance and resistance training on forkhead box O1 (FoxO1) mediated transcription. 72

Figure 1.20 Putative adaptive pathways in response to endurance- and resistance-like stimuli. 73

Figure 2.1 Representative schematic of high-frequency "stacking" and conventionalfrequency resistance training protocols.

Figure 2.2 Muscle glycogen content (A, B) and 5' adenosine monophosphate activated protein kinase $^{\text {thr172 }}$ (AMPK) phosphorylation $(C, D)$ .84

Figure 2.3 Phosphorylated $\mathrm{AKT}^{\text {ser473 }}(\mathrm{A}, \mathrm{B})$ and mammalian target of rapamycin ${ }^{\operatorname{ser} 2448}$ (mTOR; C, D) relative to total, and FKHR ${ }^{\mathrm{ser} 256}$ (FoxO1) phosphorylation (E, F) .85

Figure 2.4 Eukaryotic initiation factor 4E-binding $\operatorname{protein}^{\text {thr70 }}$ (4E-BP1; A, B)

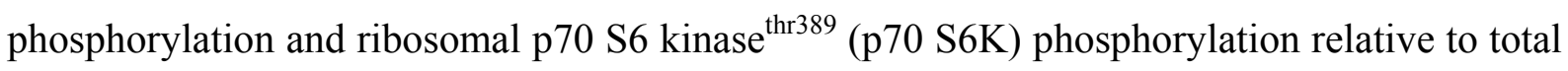
(C, D)

Figure 2.5 Total tumor necrosis factor alpha $(\mathrm{TNF} \alpha)$ content $(\mathrm{A}, \mathrm{B})$, phosphorylated $\mathrm{p} 38^{\text {thr180 }}$ mitogen activated protein kinase (MAPK) relative to total $(\mathrm{C}, \mathrm{D})$, and inhibitor kappa $\mathrm{B}$ kinase $^{\text {ser180/181 }}(\mathrm{IKK})$ phosphorylation (E, F) 88

Figure 3.1' 5 adenosine monophosphate kinase (AMPK) phosphorylation.... 105

Figure 3.2 AKT (A, B) and tuberous sclerosis complex-2 (TSC2; C, D) phosphorylation .. 106 Figure 3.3 Eukaryotic initiation factor 2B (eIF2B; A, B) and ribosomal p70 S6 kinase (p70 S6K; C, D) phosphorylation 107

Figure 3.4 Extracellular regulating kinase (ERK; A, B) and p38 (C, D) MAPK phosphorylation 109

Figure 4.1 Changes in mRNA abundance for genes associated with endurance responses .. 127 
Figure 4.2 Changes in mRNA abundance for genes associated with myogenic responses... 128

Figure 4.3 Changes in mRNA abundance for genes associated with endurance responses .. 129

Figure 4.4 Changes in mRNA abundance for genes associated with myogenic responses... 130 


\section{List of Tables}

Table 1.1 Summary of research investigating AKT responses to contractile overload in skeletal muscle.

Table 1.2 Summary of research investigating AKT responses to exercise in skeletal muscle. 30

Table 1.3 Summary of research investigating MAPK responses to exercise in vivo human skeletal muscle. 46

Table 1.4 Summary of research investigating the effect of concurrent strength and endurance training on skeletal muscle adaptation. 68

Table 3.1 Subject characteristics. 99

Table 4.2 Primer sequences and concentrations used for real-time PCR. 124 


\section{Abbreviations}

ACC acetyl-CoA carboxylase

AMP adenosine monophosphate

AMPK 5' adenosine monophosphate kinase

AP1 activator protein 1

AS160 AKT substrate of $160 \mathrm{kDa}$

ATP adenosine triphosphate

BCAA branch chain amino acid

$\mathrm{CaN} \quad$ calcineurin

CaMK calmodulin kinase

CAMKK calmodulin kinase kinase

CHO carbohydrate

COX cytochrome $\mathrm{C}$ oxidase

DNA deoxyribose nucleic acid

eEF eukaryotic elongation factor

Egr early growth response gene-1

eIF eukaryotic initiation factor

ERK extra-cellular regulating factor

ERR estrogen-related receptor alpha

FoxO Forkhead box $\mathrm{O}$

FT fast twitch

G $\beta \mathrm{L} \quad$ G beta $\mathrm{L}$ protein

Glut4 glucose transporter 4

GSK glycogen synthase kinase

HDAC histone deacteylase

HFES high-frequency electrical stimulation 


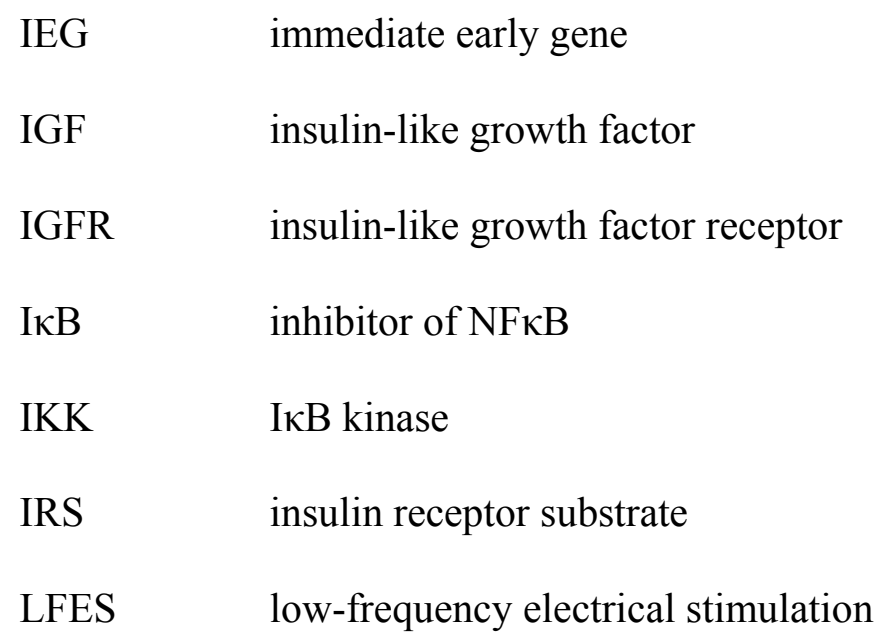

MAFBx muscle atrophy $\mathrm{F}$ box protein

MAPK mitogen activated protein kinase

MEF myocyte enhancer factor

MEK mitogen activated kinase

Mfn mitofusin

MK mitogen protein kinase

MNK MAPK-interacting kinase

MRF myogenic regulatory factor

mRNA messenger ribose nucleic acid

MSK mitogen and stress activated kinase

mTOR mammalian target of rapamycin

MuRF muscle ring finger protein

MyoG myogenin transcription factor

MyoD myogenic differentiation transcription factor

NAD/NADH nicotinamide adenine dinucleotide/hydroxide

$\mathrm{NF \kappa B} \quad$ nuclear factor kappa enhancer binding protein

NRF nuclear respiratory factor

PDK1 3' phosphoinositide-dependent protein kinase 1

PDK4 pyruvate dehydrogenase kinase 4 
PIP2 phosphatidylinositol-3-OH kinase phosphatidylinositol-4, 5 biphosphate

PIP3 phosphatidylinositol-3-OH kinase phosphatidylinositol-3, 4, 5 triphosphate

PI3K phosphatidylinositol-3-OH kinase

PPAR peroxisome proliferator activated receptor

PGC-1 $\alpha$ peroxisome proliferator activated receptor gamma co-activator-1 alpha

PHLPP PH domain leucine-rich repeat protein phosphatase

PTEN lipid-binding phosphatase and tensin homologue

p70 S6K ribosomal protein p70 S6 kinase

PWC peak work capacity

Rheb Ras homologue enriched in brain

RM repetition maximum

ROS reactive oxygen species

rpS6 ribosomal protein S6

RSK ribosomal S6 kinase

SHP2 SH2-containing inositol 5'-phosphatase-2

S6K ribosomal protein S6 kinase

ST slow twitch

TAK transforming growth factor beta activated kinase

TBS tris-buffered saline

TBST tris-buffered saline with tween

TFAM mitochondrial transcription factor $\mathrm{A}$

TG transgenic

$\mathrm{TNF} \alpha \quad$ tumor necrosis factor alpha

TNFR tumor necrosis factor receptor

TRAF TNF-receptor-associated factor

TSC tuberous sclerosis complex 
UCP uncoupling protein

VEGF vascular endothelial growth factor

$\mathrm{VO}_{2 \text { peak }} \quad$ maximal oxygen uptake

$\mathrm{VO}_{2 \text { peak }} \quad$ peak oxygen uptake

WT wild type

4E-BP1 eukaryotic initiation factor 4E-binding protein 


\begin{abstract}
The molecular events that promote or inhibit specific training adaptations (i.e. skeletal muscle hypertrophy or mitochondrial biogenesis) are not completely understood. Accordingly, there is a need to better define both the acute and chronic responses to divergent exercise stimuli in order to elucidate the specific molecular mechanisms that ultimately determine skeletal muscle phenotype. Therefore, the primary aims of the studies undertaken for this thesis were to examine the acute molecular adaptation responses in skeletal muscle following resistance and endurance training.
\end{abstract}

In order to determine the acute molecular events following repeated bouts of exercise, the study described in Chapter Two compared a high-frequency "stacked" training regimen designed to generate a summation of transient exercise-induced signalling responses with a conventional-frequency resistance training protocol. Groups $(n=6)$ of Sprague-Dawley rats performed either high-frequency training (four exercise bouts consisting of $3 \times 10$ repetitions separated by $3 \mathrm{~h}$ ) or conventional-frequency training (three exercise bouts consisting of $4 \times 10$ repetitions with $48 \mathrm{~h}$ between sessions). Protocols were matched for total work, and repetitions were performed at $75 \%$ one-repetition maximum with 3 min recovery between sets. White quadriceps muscle was extracted $3 \mathrm{~h}$ after every training bout, and 24 and $48 \mathrm{~h}$ following the final exercise session of each protocol. AKT phosphorylation was significantly decreased $3 \mathrm{~h}$ following the $2 \mathrm{nd}$ bout of high-frequency training, an effect that persisted until $48 \mathrm{~h}$ after the final exercise bout $(\mathrm{P}<0.05)$, while the phosphorylation state of this kinase was unchanged with conventional training. These results suggest that high-frequency training suppressed IGF-1 mediated signalling. Furthermore, high-frequency training generated sustained and coordinated increases in TNF $\alpha$ and IKK phosphorylation $(\mathrm{P}<0.05)$, indicating an extended response of inflammatory signalling pathways. Conversely, and irrespective of an initial increase after the first bout of exercise, TNF $\alpha$ signalling ultimately returned to control 
values by DAY 5 of conventional-frequency training, indicative of a rapid adaptation to the exercise stimulus. Notably, despite differential AKT activation there were similar increases in p70 S6K phosphorylation with both training protocols. These results indicate high-frequency resistance training extends the transient activation of inflammatory cytokine-mediated signalling and results in a persistent suppression of AKT phosphorylation, but these events do not appear to inhibit kinase activity proximal to translation initiation.

The aim of the study described in Chapter Three was to determine the effect of prior training history on selected signalling responses after an acute bout of resistance and endurance exercise. Following $24 \mathrm{~h}$ diet / exercise control 13 male subjects ( 7 strength-trained and 6 endurance-trained) performed a random order of either resistance $(8 \times 5$ maximal leg extensions) or endurance exercise ( $1 \mathrm{~h}$ cycling at $70 \%$ peak $\mathrm{O}_{2}$ uptake). Muscle biopsies were taken from the vastus lateralis at rest, immediately and $3 \mathrm{~h}$ post-exercise. AMPK phosphorylation increased after cycling in strength-trained, but not endurance-trained subjects $(\mathrm{P}<0.05)$. Conversely, AMPK was elevated following resistance exercise in endurance-, but not strength-trained subjects $(\mathrm{P}<0.05)$. Thus, AMPK was elevated only when subjects undertook a bout of exercise in a mode of training to which they were unaccustomed. Surprisingly, there was no change in AKT phosphorylation following resistance exercise regardless of the training background of the subjects. In the absence of increased AKT phosphorylation, resistance exercise induced an increase in p70 S6K and ribosomal S6 protein phosphorylation in endurance-trained but not strength-trained subjects $(\mathrm{P}<0.05)$. AKT phosphorylation was increased in endurance-trained, but not strength-trained subjects after cycling $(\mathrm{P}<0.05)$. These results show that a degree of signalling "response plasticity" capable of diverse adaptive compliance is conserved at opposite ends of the adaptation continuum. Furthermore, the adaptive phenotype associated with a prolonged training history alters the subsequent signalling responses with divergent exercise stimuli. 
The third study described in Chapter Four quantified the acute sub-cellular mRNA responses in muscle to habitual and unfamiliar exercise modes. This study employed the same subjects and protocols outlined in Chapter Three and analysis was performed on muscle biopsies taken at rest and $3 \mathrm{~h}$ after an exercise bout. Gene expression was analysed using Real-Time PCR with changes normalised relative to pre-exercise values. Following cycling exercise peroxisome proliferator activated receptor gamma co-activator-1 alpha, pyruvate dehydrogenase kinase 4 and vascular endothelial growth factor mRNA abundance was significantly increased in both endurance and strength-trained subjects $(\mathrm{P}<0.05)$. This finding indicates that the adaptive phenotype of these athletes did not produce a disparity in the mRNA response of these 'metabolic genes'. Similarly, muscle atrophy F box protein (MAFBx) mRNA increased in both groups $(\mathrm{P}<0.05)$ suggesting that a prolonged endurance training stimulus may increase the activity of pathways involved in the regulation of muscle atrophy. Unexpectedly, MyoD and Myogenin mRNA increased in endurance-trained subjects after cycling. Accordingly, it seems plausible to suggest that these regulators of satellite cell activity may have additional roles in skeletal muscle metabolism. Finally, high-intensity resistance exercise did not induce any change in mRNA abundance of selected 'myogenic' genes in either group of subjects, despite a decrease in MAFBx and Myostatin mRNA in endurance-trained subjects. It may be that in highly-trained athletes a greater volume or repeated bout effect is required to initiate anabolic gene expression. Taken collectively, these results indicate that prior training history can modify the acute mRNA changes in skeletal muscle in response to exercise. Indeed, the data indicate that independent of exercise mode, the gene responses in skeletal muscle from endurance-trained athletes are more sensitive to alterations in the cellular milieu than strength-trained subjects.

In summary, the work from the studies undertaken for this thesis provides novel information regarding the effects of the frequency of the training stimulus on signalling responses in skeletal muscle. Specifically, high-frequency resistance training does not 
enhance anabolic signal transduction and may exacerbate inflammation and atrophy. In regard to the effects of prior contractile history on early signalling and mRNA responses, divergent exercise induces a mode specific molecular response that is altered by the training-induced phenotype of the muscle, highlighting the need for extensive training overload in highlytrained athletes. 


\section{Chapter One}

Literature Review 


\subsection{Overview: The Specificity of Exercise Responses}

Skeletal muscle is a malleable tissue capable of altering the type and amount of protein in response to disruptions to cellular homeostasis. Contractile activity as a result of physical activity (i.e. exercise) represents a potent stimulus that can generate significant disturbance within the muscle milieu and elicit substantial changes and adjustment in the cellular environment. Indeed, repeated contractile activity produces a cascade of events that ultimately alters the adaptive machinery and tissue characteristics resulting in less interference to cell homeostasis during subsequent contractions (Figure 1.1).

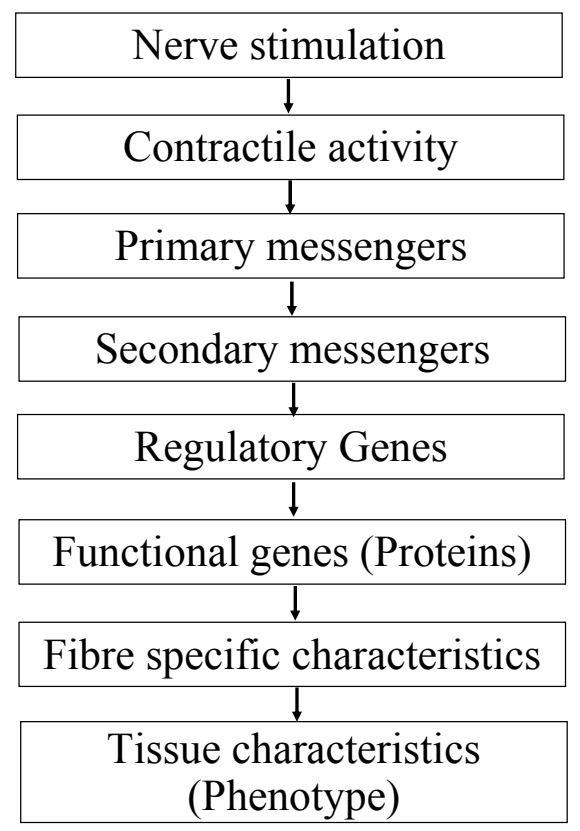

Figure 1.1 Putative steps through which contractile activity leads to alterations of skeletal muscle (Williams and Neufer 1996).

Current understanding of the complex process of exercise-induced adaptation in skeletal muscle was pioneered in the 1960's. Notably, Holloszy (1967) was the first exercise scientist to utilise conventional biochemistry techniques to investigate the effects of exercise on skeletal muscle. Holloszy (1967) established that regular prolonged exercise significantly increased skeletal muscle mitochondrial content and enzyme activity, thereby elucidating a molecular mechanism for the increase in aerobic work capacity with endurance training. 
Similarly, Goldberg (1968) pioneered work that identified the capacity of skeletal muscle to increase in size in response to physiological overload. He showed that overload-induced compensatory hypertrophy occurred via the incorporation of amino acid derived protein synthesis, the principal molecular event to increase muscle mass with resistance training. Since these early studies advances in biotechnology have enabled the elucidation of specific signalling mechanisms that initiate replication of DNA sequences and translation of the genetic message into a series of amino acids that create new protein (Bouchard et al. 1997). The continuous turnover of cell proteins and alterations in the equilibrium between protein synthesis and degradation permits muscle plasticity (Booth and Baldwin 1996). The functional consequences of these exercise-induced adaptation processes are determined by the volume, intensity and frequency of the stimulus and the half-life of the protein. Moreover, many features of the resultant adaptation after repeated bouts of contractile activity are specific to the type of stimulus, such as the mode of exercise (Williamson et al. 2001; Izquierdo et al. 2004; Mahoney et al. 2005).

The early signalling responses elicited by a single bout of exercise generate increases in transcription of nuclear / mitochondrial DNA resulting in greater mRNA abundance of specific exercise-induced genes. While gene expression and subsequent protein synthesis in response to exercise is regulated at multiple steps, the directional change in mRNA is generally the same as the protein during early adaptation to a new steady-state level (Booth and Baldwin 1996). Contraction generates transient increases in the quantity of mRNA for specific exercise-induced genes commonly peaking between 3-12 h post-exercise and returning to basal levels within 24 h (Figure 1.2a; Pilegaard et al. 2000; Psilander et al. 2003; Bickel et al. 2005; Yang et al. 2005). Therefore, frequent repeated bouts of exercise will result in increased transcriptional activity that subsequently amplifies protein synthesis. Consequently, chronic adaptation to training is likely due to the cumulative effects of each acute exercise bout leading to a change in the steady-state level of specific proteins and a new 
functional threshold (Figure 1.2b; Widegren et al. 2001; Mahoney et al. 2005; Pilegaard et al. 2005).
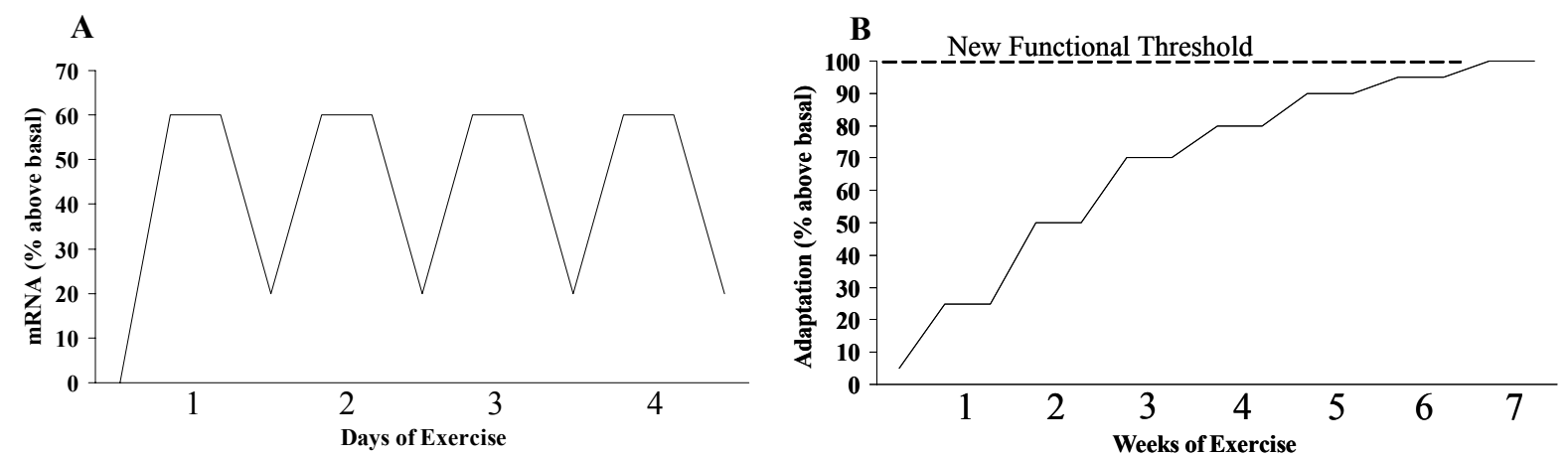

Figure 1.2 Schematic of the putative (A) mRNA and (B) protein responses with adaptation to repetitive exercise stress.

Diverse exercise stimuli induce specific early gene responses and protein synthesis in skeletal muscle (Atherton et al. 2005). Individuals have different genetic capacity to perform and adapt to aerobic or anaerobic exercise (Bouchard et al. 2000) and muscle fibre composition is an important contributing factor to this process. Nevertheless, while the mechanisms that transduce the fibre-type specific effect of contractile activity are incompletely understood, numerous signalling proteins and genes have been implicated in exercise-induced adaptation (Hawley 2002; Glass 2005; Koulmann and Bigard 2006). Prolonged endurance training elicits a variety of metabolic and morphological gene expression including those related to mitochondrial biogenesis (Holloszy 1967; Irrcher et al. 2003), fast-slow fibre-type transformation (Zierath and Hawley 2004) and substrate metabolism (Holloszy et al. 1977). In contrast, heavy resistance exercise stimulates adaptive machinery responsible for increasing muscle hypertrophy (Rennie et al. 2004) and maximal contractile force output (Häkkinen 1989; Ahtiainen et al. 2003).

Concomitant with the vastly different functional outcomes induced via these diverse exercise modes, the genetic and molecular mechanisms of adaptation are distinct and may be 
regarded as incompatible. Each mode of exercise results in activation and / or repression of specific pathways and subsets of genes. Performing multiple bouts of each training mode in isolation will ultimately generate a developmental history in the muscle fibres, producing a specific exercise-induced phenotype associated with chronic training (Ingalls 2004). Therefore, aerobic endurance and heavy resistance training represent opposite ends of an adaptation continuum. While the cellular and molecular events associated with endurance and resistance exercise adaptations are becoming evident, our current understanding of the adaptation process when performing concurrent endurance and resistance training is less clear. Previous work (Leveritt et al. 2003) suggests that concurrent endurance and resistance training reduces the adaptive response compared to when each exercise mode is undertaken alone (Figure 1.3).

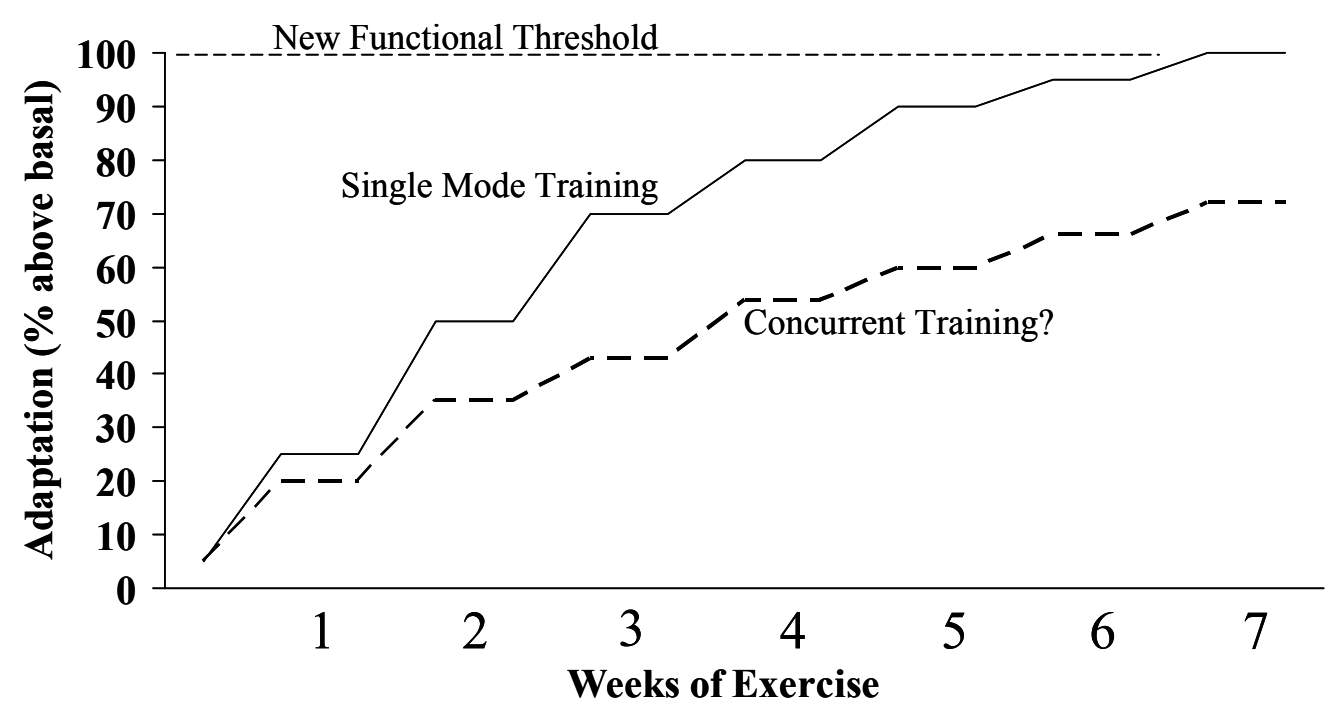

Figure 1.3 Proposed effect of concurrent training on specific adaptation when compared to single mode training.

Metabolic and mitochondrial adaptation to aerobic exercise is typically associated with resistance to lifestyle related diseases, while increased muscular strength and power may prevent musculo-skeletal injury and loss of function. Greater knowledge of the mechanisms 
and interaction of exercise-induced adaptive pathways in skeletal muscle is important for our understanding of the aetiology of disease, maintenance of metabolic and functional capacity with aging, and training for athletic performance. This chapter will provide a detailed review of adaptive pathways in skeletal muscle and highlight the effects of exercise on genetic and molecular mechanisms of adaptation. 


\subsection{Skeletal Muscle Mechanotransduction}

The process of converting a mechanical signal to a biochemical event in a muscle cell has been termed mechanotransduction (Hornberger and Esser 2004) and involves the upregulation of primary and secondary messengers that initiate a cascade of events that result in activation and / or repression of specific signalling pathways regulating exercise-induced gene expression and protein synthesis / degradation (Williams and Neufer 1996). One of the most potent stimuli for skeletal muscle mechanotransduction is contraction.

\subsubsection{Putative Primary Messengers}

Elucidation of the precise mechanisms that enable skeletal muscle cells to interpret and respond to contraction has proved elusive. Indeed, the complexity of this process is complicated by 1) the proposed mechanoreceptors that connect the neuronal, mechanical and biochemical events and 2) the numerous cellular candidates as potential primary messengers that transduce the mechanical signal. In addition, it is unlikely that these primary signalling messengers act in isolation and probably results in an intricate multifaceted signal with redundancy and cross-talk. Nevertheless, a number of putative messengers is emerging including, but not limited to, calcium, $\mathrm{Na}^{+} / \mathrm{K}^{+}$, mechanical stretch, $\mathrm{pH}$, hydrogen ions, redox state, hypoxia, and phosphorylation state. This review will focus on several important primary messengers that have been identified to be altered with exercise-induced contractile activity (Williams and Neufer 1996; Sakamoto and Goodyear 2002; Hawley and Zierath 2004).

\section{Mechanical Stretch}

Mechanical stimuli modulate cell function via membrane distortion and mechanochemical conversion, and directly affect tissue form and function (Alenghat and Ingber 2002). Putative mechanoreceptors for signal transduction in skeletal muscle include the phospholipid bilayer 
and surface adhesion receptors (Hornberger and Esser 2004). Movement of intra- and extracellular components via disruption to the lipid membrane and activation of focal adhesion kinases through integrin receptors as a result of muscle contraction represent likely candidates that activate secondary events (Hornberger and Esser 2004; Rennie et al. 2004). Technical limitations currently prevent accurate measurement of the effect of mechanical stimuli on mechanotransduction in vivo. However, the use of passive stretch on muscle in vitro and in situ demonstrates that mechanical stimuli induce numerous adaptive processes. Passive stretch has been shown to alter myogenic regulatory factors (MRF), myosin heavy chain and fast-to-slow phenotype gene expression in both innervated and denervated skeletal muscle (Loughna et al. 1990; Loughna and Morgan 1999; Rauch and Loughna 2005). Furthermore, stretch is capable of generating significant increases in skeletal muscle insulin-like growth factor (IGF) mRNA and hypertrophy (Czerwinski et al. 1994).

Evidence is emerging that mechanical perturbation of skeletal muscle mediates activation of the calcineurin, mitogen activated protein kinase (MAPK) and IGF signalling cascades (Kumar et al. 2002; Hornberger et al. 2005a; Rauch and Loughna 2005). Moreover, it has become apparent that muscle cells can distinguish between mechanical forces acting on the cell. Work by Kumar and colleagues (2002) revealed that axial versus transverse stress produced activation of distinct signalling intermediates despite the same magnitude of mechanical stress applied to the muscle fibre. Similarly, Hornberger and co-workers (2005a) observed unique activation of signalling proteins when comparing cyclic uni- and multi-axial stretch. The rapid and differentiated mechanochemical conversion induced by distinct models of mechanical stress strongly suggests the existence of mechanotransduction specificity. Therefore, the signalling events initiated by mechanical load with exercise (i.e. frequency and intensity of contraction) are likely to contribute to the specificity of exercise-induced adaptation and implicates mechanical stress / tension as a significant primary messenger. 


\section{Calcium}

Neural activation of skeletal muscle generates an action potential that results in $\mathrm{Ca}^{2+}$ release from the sarcoplasmic reticulum (SR) via $\mathrm{Ca}^{2+}$ release channels triggering a rise in cellular $\left[\mathrm{Ca}^{2+}\right]$ for excitation-contraction coupling (Carroll et al. 1995). Cessation of the action potential initiates the transport of $\mathrm{Ca}^{2+}$ out of the cytosol back to the sarcoplasmic reticulum. The rate and capacity of $\mathrm{Ca}^{2+}$ release and uptake is altered by contractile activity (Figure 1.4).

Prolonged moderate intensity $\left(\sim 60-70 \% \mathrm{VO}_{2 \max }\right)$ exercise has been shown to increase $\mathrm{SR} \mathrm{Ca}^{2+}$ uptake and may increase the number of active pumps resequestering $\mathrm{Ca}^{2+}$ (Schertzer et al. 2003; Schertzer et al. 2004). In contrast, a single bout of high-intensity $\left(>100 \% \mathrm{VO}_{2 \max }\right)$ or fatiguing exercise generates a $20-50 \%$ transient decrease in $\mathrm{Ca}^{2+}$ uptake and release, returning to basal levels following 60 min recovery (Byrd et al. 1989; Matsunaga et al. 2002). These acute alterations in cytosolic $\left[\mathrm{Ca}^{2+}\right]$ may elicit secondary events following a return to resting / basal values. Interestingly, repeated bouts of exercise have been shown to induce an adaptive response resulting in less perturbation in $\mathrm{Ca}^{2+}$ release and uptake, and subsequently improved $\mathrm{Ca}^{2+}$ cycling and resistance to fatigue (Holloway et al. 2005). Taken together, these findings provide evidence to suggest the amplitude and duration of $\mathrm{Ca}^{2+}$ flux is regulated by the duration and frequency of contractile stimulus. For example, endurance exercise likely results in extended periods of moderately elevated $\left[\mathrm{Ca}^{2+}\right]$, while resistance exercise would generate short cycles of significantly higher intra-cellular $\left[\mathrm{Ca}^{2+}\right]$ (Baar et al. 1999). Indeed, it is plausible to suggest that the specific cytosolic $\mathrm{Ca}^{2+}$ transient will also determine subsequent downstream events such as gene expression and protein synthesis (Chin 2005; Tidball 2005).

$\mathrm{Ca}^{2+}$ cycling has been implicated in the activation of multiple potential secondary messenger complexes including calcineurin, nuclear factor of activated T-cells (NFAT), calmodulin, MAPK and protein kinase C (Williams and Neufer 1996; Baar et al. 1999; Sakamoto and Goodyear 2002). Elevation of intranuclear $\left[\mathrm{Ca}^{2+}\right]$ may also result in activation of histone deacetylase (HDAC) and myocyte enhancer factor 2 (MEF2) (Liu et al. 2005). 
However, the precise relationship between alterations in $\left[\mathrm{Ca}^{2+}\right]$ and initiation of the adaptive processes culminating in gene expression and protein synthesis remains elusive. Nevertheless, $\mathrm{Ca}^{2+}$ appears to be a compelling regulator in the specificity of exercise-induced adaptive events and its effects are likely to be altered by the mode, intensity and volume of exercise.

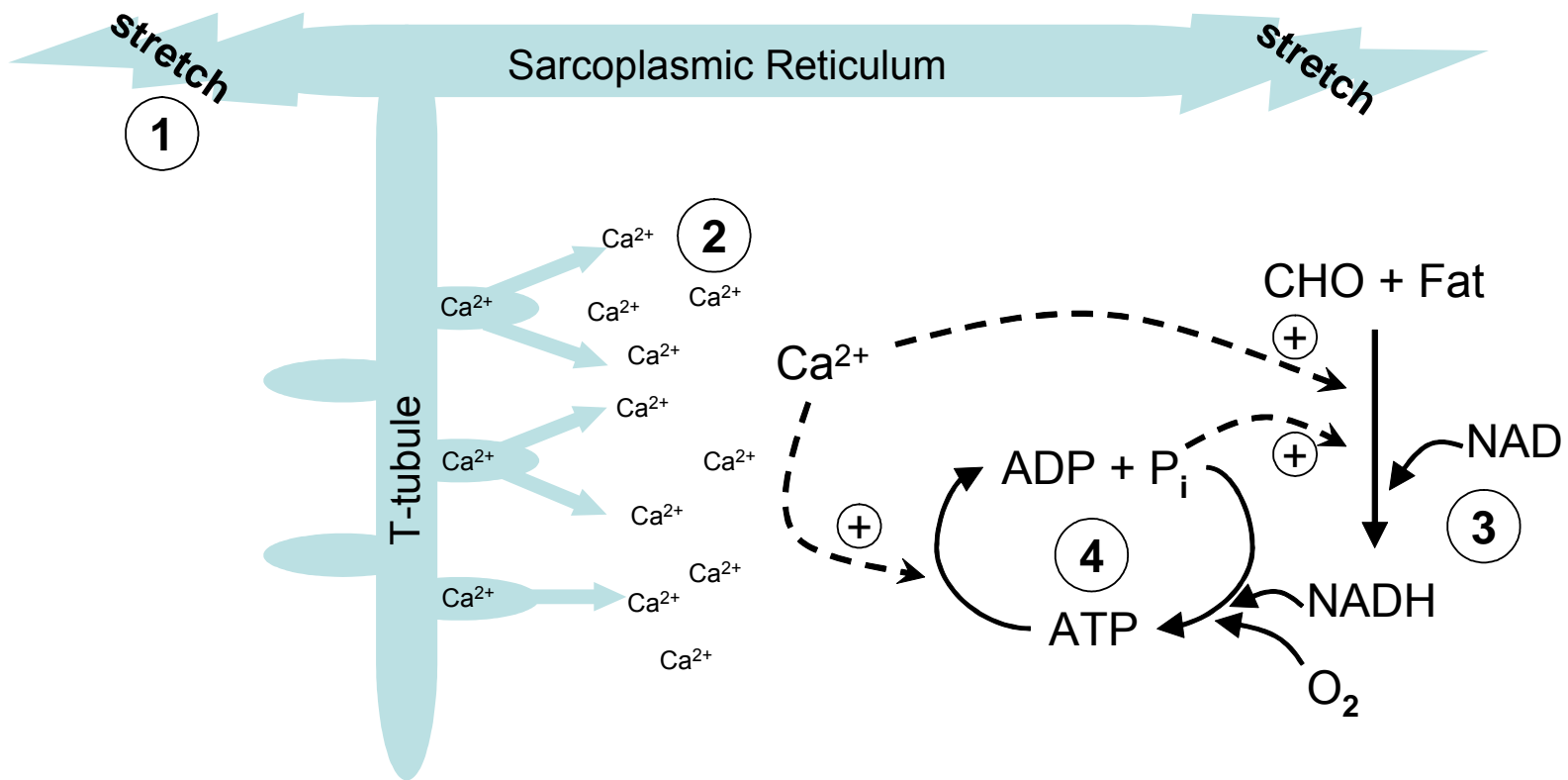

Figure 1.4 Metabolic and mechanical signals believed to play key roles as primary messengers in mechanotransduction. 1) Mechanical stretch 2) $\mathrm{Ca}^{2+}$ flux 3) redox potential and 4) phosphorylation potential. $\mathrm{Ca}^{2+}$, calcium; $\mathrm{CHO}$, carbohydrate (Spriet 2002; Hawley and Zierath 2004).

\section{Redox Potential}

The process of maintaining redox potential occurs when nicotinamide adenine dinucleotide (NAD) is converted to NADH, and to maintain redox homeostasis NADH must be constantly re-oxidised via several different pathways (Lin and Guarente 2003). This redox mechanism is predominantly a result of the catabolic reactions occurring with glycolytic and lipolytic metabolism (Figure 1.4). Therefore, the NAD/NADH ratio regulates intra-cellular redox state 
and may be considered a marker of skeletal muscle metabolic state (Lin and Guarente 2003). Indeed, contractile function in the absence of fatigue and during strenuous exercise to exhaustion is modulated moment-to-moment by cellular redox state (Smith and Reid 2006).

The maintenance of redox potential produces volatile free oxygen molecules (e.g. reactive oxygen species [ROS]) and this oxidant activity is buffered by multiple anti-oxidant systems in skeletal muscle (Stofan et al. 2000; Arbogast and Reid 2004). Due to the increase in demand for oxygen and activity of metabolic pathways, exercise represents a stimulus capable of generating high levels of ROS. While a direct cause and effect relationship has not been established, indirect evidence suggests that oxidative stress limits exercise capacity and may modulate exercise-induced adaptive signalling. ROS-selective antioxidants have been utilised to significantly increase sub-maximal exercise time to exhaustion but do not appear to promote any delay in fatigue at high-intensity workloads in human skeletal muscle in vivo (Medved et al. 2003; Medved et al. 2004; Matuszczak et al. 2005). Therefore, prolonged endurance exercise appears to be a stimulus more likely to promote oxidative stress and ROS activity than heavy resistance training. This may characterise a point of diversion in the specificity of exercise-induced signalling.

Our present understanding indicates that redox potential and resultant free radical synthesis with exercise and during recovery may regulate adaptive pathways in two ways. In the first instance, redox state may act as a primary messenger through a direct affect on transcriptional regulation and DNA binding specificity of immediate early genes (IEG's) such as Fos and Jun, and transcription factors nuclear factor kappa $\mathrm{B}(\mathrm{NF \kappa \textrm {B }})$ and activator protein 1 (AP1; Xanthoudakis et al. 1992; Carrero et al. 2000; Jindra et al. 2004). In addition, due to the apparent effect of ROS on numerous elements of cellular function redox state may act indirectly on signalling machinery via its effect on intra-cellular $\mathrm{Ca}^{2+}$ regulation and mitochondrial metabolism (Smith and Reid 2006). 


\section{Phosphorylation Potential}

Muscle contraction is an energy consuming process that requires the continual hydrolysis of adenosine triphosphate (ATP) to sustain force production. Resynthesis of ATP via restoration of its high energy phosphate bonds is generated by oxidative phosphorylation and / or glycolysis (Houston 2001). Subsequently, concentrations of metabolites related to the maintenance of muscle phosphorylation potential (i.e. [ATP]/[ADP][Pi]) provide feedback signals to balance ATP production with ATP consumption (Hawley and Zierath 2004). Indeed, intra-cellular concentrations of free adenosine monophosphate (AMP) are an important regulator of energy consuming and regenerating pathways (Sakamoto and Goodyear 2002).

Contraction has been clearly shown to alter metabolite concentration in both type I and type II muscle fibres (Hintz et al. 1982; Ivy et al. 1987). Strong evidence also exists for the relationship between metabolite concentrations and contractile intensity and duration during both continuous and intermittent models of electrical stimulation and in vivo exercise (Hintz et al. 1982; Ivy et al. 1987; Bangsbo et al. 2001; Ferguson et al. 2001; Krustrup et al. 2003). Moreover, the maintenance of phosphorylation potential appears to be inversely related to exercise intensity. Work by Krustrup and colleagues (2003) revealed significant differences in ATP and creatine phosphate (CP) degradation in response to either low $\left(50 \% \mathrm{VO}_{2 \text { peak }}\right)$ or high $\left(110 \% \mathrm{VO}_{2 \text { peak }}\right)$ intensity exercise, in which the more intense exercise generated a $20 \%$ greater degradation in muscle ATP concentration. Likewise, Ivy and co-workers (1987) have shown a concomitant decrease in $\mathrm{CP}$ concentration as exercise intensity increases during a graded exercise protocol to exhaustion. Exercise duration also results in modulation of phosphorylation potential whereby exercise at a constant work rate has been shown to increase the rate of ATP turnover, reducing mechanical efficiency with continued exercise (Bangsbo et al. 2001). 
Any cellular stress that inhibits ATP synthesis or accelerates ATP consumption (e.g. exercise-induced contraction) and subsequently increases the AMP:ATP ratio potentially initiates numerous downstream molecular events in skeletal muscle (Hardie and Sakamoto 2006). As a primary messenger for adaptive signalling, phosphorylation state appears to principally exert its effect via a potent secondary messenger, the 5'adenosine monophosphate activated protein kinase (AMPK; Sakamoto and Goodyear 2002; Hawley and Zierath 2004; Hardie and Sakamoto 2006). Therefore, phosphorylation potential and subsequent AMPK activation may ultimately regulate multiple cell signalling cascades for such diverse processes as glucose uptake, fatty acid oxidation, hypertrophy and gene expression (Aschenbach et al. 2004). This ubiquitous kinase is discussed at greater length in section 1.2.2.

\section{Summary}

All mechanotransduction events are important in coordinating and transmitting the mechanochemical signals produced during exercise. Collectively, and in a coordinated manner, these signals provide the mechanistic framework by which exercise initiates and regulates intra-cellular signal transduction to the transcriptional machinery in the nucleus, modulating gene expression and ultimately protein turnover, generating an exercise specific signal.

\subsubsection{Secondary Messengers}

Following initiation of the primary signal additional kinases / phosphatases are activated to mediate the exercise-induced signal. Numerous signalling cascades exist in mammalian cells and these pathways are highly regulated at multiple levels with substantial cross-talk between pathways producing a highly sensitive, complex transduction network. This review will consider major pathways of contraction and growth factor signalling in skeletal muscle. 


\section{5'Adenosine Monophosphate Activated Protein Kinase (AMPK) Mediated Signalling}

AMPK is a heterotrimeric protein consisting of three subunits $(\alpha, \beta, \gamma)$, and functions as a key regulator of energy homeostasis in skeletal muscle (Winder 2001; Aschenbach et al. 2004; Hardie and Sakamoto 2006; Witters et al. 2006). Each subunit of AMPK exists in multiple isoforms and its presence in skeletal muscle may be unique, in that it appears to be the only tissue containing all catalytic $\alpha$-subunit $(\alpha 1, \alpha 2)$ and regulatory $\beta$ - $(\beta 1, \beta 2)$ and $\gamma$-subunit $(\gamma 1$, $\gamma 2, \gamma 3$ ) isoforms (Hardie and Sakamoto 2006). AMPK is directly activated by 5 'AMP and consequently is sensitive to changes in cellular AMP:ATP ratios (Carling et al. 1987; Hardie et al. 1999). In addition, upstream kinases LKB1 and calcium calmodulin-dependent kinase kinase (CAMKK) also converge at threonine ${ }^{172}$ on the $\alpha$-subunit to phosphorylate and activate AMPK in response to altered cellular AMP and $\mathrm{Ca}^{2+}$, respectively (Hawley et al. 2005; Hurley et al. 2005; Sakamoto et al. 2005; Woods et al. 2005; Witters et al. 2006).

Acute activation of AMPK in response to cellular energy depletion (e.g. skeletal muscle contraction) initiates actions to both conserve and generate ATP (Witters et al. 2006). AMPK is implicated in enhancing ATP production by stimulating insulin-independent glucose uptake (Hayashi et al. 1998; Musi et al. 2001; Nakano et al. 2006) and increasing fat oxidation, primarily via inhibition of the $\beta$ isoform of acetyl-CoA carboxylase (ACC- $\beta$ ) thereby reducing the synthesis and inhibitory effects of malonyl-CoA and subsequently increasing fatty acyl-CoA translocation into the mitochondria (Merrill et al. 1997; Kaushik et al. 2001; Aschenbach et al. 2004; Lee et al. 2006). Additional effects of contraction-mediated AMPK activation are less clear but may result in multiple adaptive events including altered protein synthesis and gene expression (Figure 1.5). AMPK has been linked to control of gene expression by activating transcription factors associated with mitochondrial fatty acid oxidation and the inhibition of protein synthesis by down-regulating components of the insulin / insulin-like growth factor signalling pathway (Bolster et al. 2002; Zong et al. 2002; 
Kimura et al. 2003; Terada and Tabata 2003; Jorgensen et al. 2005; Terada et al. 2005; Lee et al. 2006).

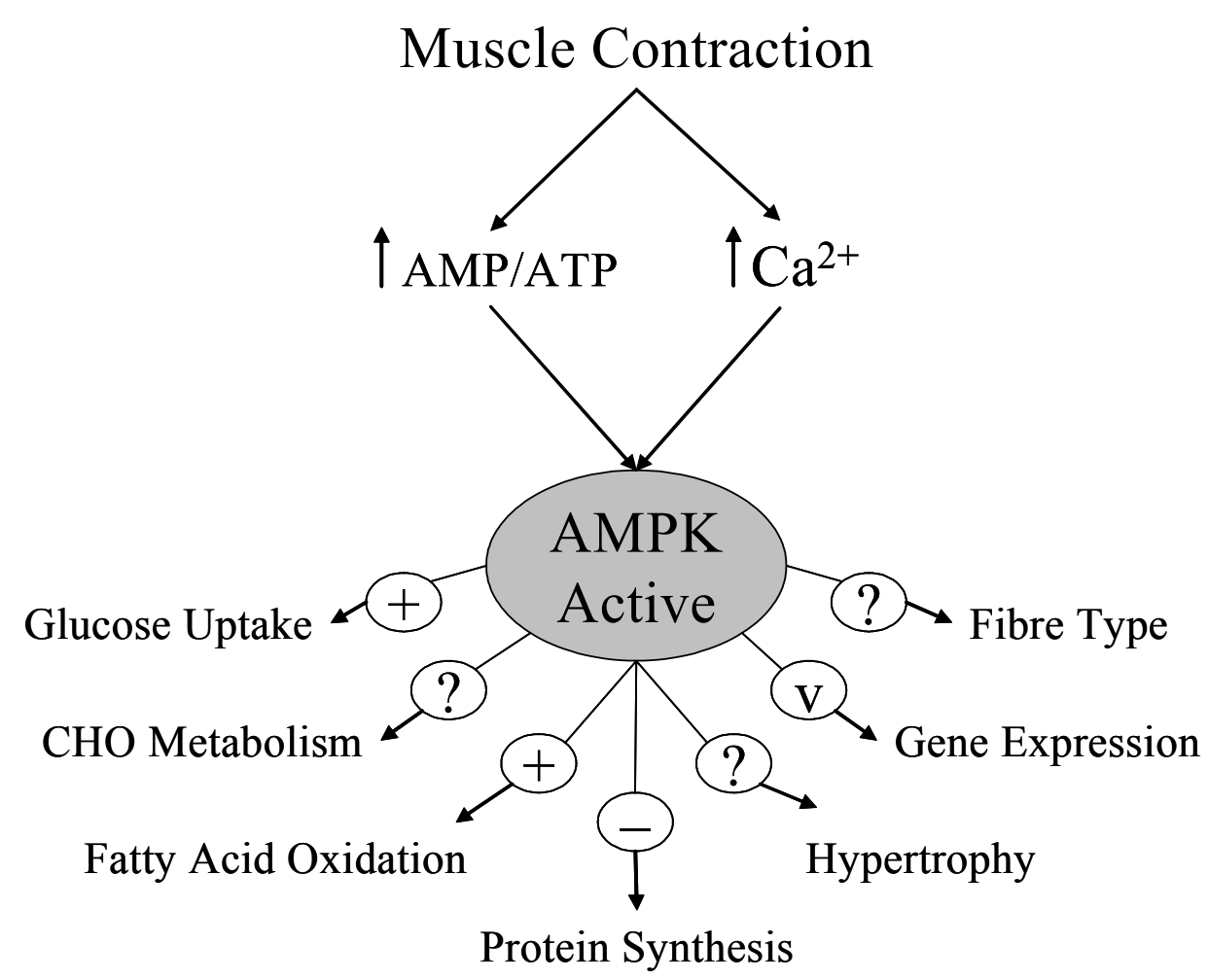

Figure 1.5 Putative contraction-mediated adaptive events associated with 5'Adenosine Monophosphate Activated Protein Kinase (AMPK) activation in skeletal muscle. + positive effect, - negative effect, V variable positive/negative effect, ? unknown effect; $\mathrm{Ca}^{2+}$, calcium; CHO, carbohydrate (adapted from Aschenbach et al. 2004 and Witters et al. 2006).

Activation of AMPK in skeletal muscle has been shown in a variety of systems including in vitro / in vivo contraction, pharmacological activators and transgenic animals (Nielsen et al. 2003b; Sakamoto et al. 2004b; Atherton et al. 2005; Jorgensen et al. 2005; Toyoda et al. 2006). Current evidence suggests that isoform specific AMPK activation occurs in a time, intensity, fibre-type and mode-specific manner and exercise-induced contraction of skeletal muscle in vivo has been clearly shown to up-regulate AMPK activity (Durante et al. 2002; Nielsen et al. 2003b; Yu et al. 2003; Frøsig et al. 2004; Atherton et al. 2005; Hurst et al. 
2005; McConell et al. 2005; Sriwijitkamol et al. 2005; Taylor et al. 2005; Wojtaszewski et al. 2005; Toyoda et al. 2006).

In vitro activation of AMPK has revealed that while increased AMPK activity occurs in both red and white muscle, $\alpha 1$ and $\alpha 2$ isoform activity is greatest in red fast-twitch muscle (Ai et al. 2002). Findings in vivo following treadmill running support the contention that AMPK activation is greater in red compared to white muscle (Rasmussen and Winder 1997; Durante et al. 2002). Importantly, interpretation of the available literature with regard to fibretype specific AMPK activity is limited because most studies have incorporated exercise protocols unlikely to significantly recruit white muscle (i.e. endurance running or swimming). However, Taylor and colleagues (2005) recently compared the effects of moderate-intensity endurance training and high-intensity interval training on AMPK signalling. They found that high-intensity interval training induced a significant additive effect to endurance training on LKB1 activity in white but not red quadriceps suggesting that white muscle may generate substantial AMPK responses when sufficiently stimulated. Nevertheless, our current understanding of fibre-type specific AMPK responses is incompletely resolved.

Commensurate with its proposed role as an 'energy sensing' kinase, AMPK activation occurs in an intensity-dependent manner and has been shown to increase with increasing contraction force, treadmill speed and cycling power (Rasmussen and Winder 1997; Ihlemann et al. 1999; Musi et al. 2001; Chen et al. 2003; Wadley et al. 2006). While acute low intensity in vitro contraction has been shown to activate AMPK $\alpha 1$ but not $\alpha 2$ isoforms (Toyoda et al. 2006), sub-maximal aerobic exercise in vivo appears to primarily increase AMPK $\alpha 2$ activity (Fujii et al. 2000; Wojtaszewski et al. 2000; Durante et al. 2002; Stephens et al. 2002; Yu et al. 2003). Indeed, endurance exercise-induced activation of AMPK is predictable given its ability to increase ATP regeneration via fat oxidation to meet the demands of prolonged lowintensity contraction. Interestingly, this exercise response seems to be dependent on the adaptive state of the muscle as evidence exists to demonstrate that short-term and chronic 
aerobic training reduces the acute AMPK response to prolonged and intermittent sub-maximal exercise (Durante et al. 2002; Yu et al. 2003; Hurst et al. 2005; McConell et al. 2005). However, the AMPK response in endurance trained athletes may be conserved with adequate overload as high-intensity interval training $\left(\sim 90 \% \mathrm{VO}_{2 \mathrm{max}}\right)$ has been shown to increase AMPK $\alpha 1$ and $\alpha 2$ in highly trained cyclists (Clark et al. 2004). Furthermore, AMPK $\alpha 1 / \alpha 2$ isoform activity and ACC phosphorylation is also significantly up-regulated following short duration $(\sim 30$ s) supra-maximal sprint cycling, likely due to the extensive and rapid reduction in cellular ATP (Chen et al. 2000).

Few studies investigating AMPK signalling have incorporated resistance training as a stimulus. Hence our knowledge of AMPK signalling following this mode of contractile activity is limited. Work by Wojtaszewski and colleagues (2005) utilised six wk of progressive overload resistance training at $50-80 \%$ of one repetition maximum (1-RM) in healthy and diabetic subjects. Their data reveal a significant increase in the protein expression of AMPK $\alpha 1, \beta 2$ and $\gamma 1$, but not $\alpha 2$ isoforms independent of the subject's health status. Interestingly, Dreyer et al. (2006) report an increase in AMPK activity after an acute bout of resistance training in humans, yet they observed increased $\alpha 2$ but not $\alpha 1$ activity. Koopman and co-workers (2006) also observed increased AMPK phosphorylation following an acute bout of resistance training, although it should be noted that the training load in that study (16 sets x 10 reps, 2 min recovery) would likely induce a high metabolic stress typically associated with endurance training. Resistance exercise-induced changes in AMPK phosphorylation may reflect an increase in insulin-independent cellular glucose uptake and transport. However, the physiological significance of increased AMPK following resistance training has yet to be determined.

Information on differences in AMPK responses between endurance and resistance training has not been extensively investigated. However, intricate work by Atherton and coworkers (2005) indicates mode-specific AMPK activation with acute bouts of "endurance- 
like" and "resistance-like" contraction. Using electrical stimulation to mimic endurance or resistance loading, these workers show increased AMPK phosphorylation immediately and 3 h post-exercise following "endurance-like" but not "resistance-like" contraction in slowtwitch soleus and fast-twitch extensor digitorum longus muscle. Therefore, given the dissimilar functional adaptive outcomes with chronic endurance versus resistance training the possibility exists that AMPK signalling is a key intermediate for the divergent adaptation with these different exercise modes.

\section{$\mathrm{Ca}^{2+}$ calmodulin-dependent kinase (CaMK) / Calcineurin Signalling}

Contraction-stimulated alterations of intra-cellular $\left[\mathrm{Ca}^{2+}\right]$ induce both direct and hierarchical signalling responses in skeletal muscle (Chin 2005; Koulmann and Bigard 2006). Specifically, calmodulin $(\mathrm{CaM})$ is an intermediate binding protein sensitive to increases in $\left[\mathrm{Ca}^{2+}\right]$ and acts as a calcium sensing unit that transduces $\mathrm{Ca}^{2+}$ flux $(>30 \mathrm{~Hz})$ via conformational changes in its protein motifs before subsequently activating downstream kinases and phosphatases (Chin 2005). Calcineurin is a $\mathrm{Ca}^{2+} /$ calmodulin-dependent phosphatase that acts as a molecular translator of $\mathrm{Ca}^{2+}$ flux activated by a sustained, low amplitude signal ( $\sim 10 \mathrm{~Hz})$ (Olson and Williams 2000; Michel et al. 2004).

The $\mathrm{Ca}^{2+}$-calmodulin-dependent serine / threonine kinases $(\mathrm{CaMK})$ detect and respond to physiologically relevant $\left[\mathrm{Ca}^{2+}\right]$ and incorporates a group of single and multi-functional kinases (Chin 2005). The specific kinases from the CaMK family found in skeletal muscle are not well defined but existing evidence indicates that CaMKII and IV are expressed in skeletal muscle (Wu et al. 2002; Rose and Hargreaves 2003). CaMKII and IV have been linked with activation of gene expression of contractile and mitochondrial proteins, respectively (Fluck et al. 2000; Wu et al. 2002). CaMKII activity has been shown to increase with stretch overload and wheel running animal models (Fluck et al. 2000) and with cycling exercise in humans (Rose and Hargreaves 2003). Indeed, it appears CaMKII is the predominant multifunctional 
$\mathrm{CaMK}$ in response to endurance exercise and is rapidly up-regulated after commencing exercise in an intensity-dependent manner (Rose et al. 2006). While transgenic animals selectively expressing CaMKIV appear to enhance mitochondrial biogenesis following electrical stimulation (Wu et al. 2002), these findings are contentious as other investigators have failed to detect CaMKIV protein in skeletal muscle (Rose and Hargreaves 2003; Akimoto et al. 2004).

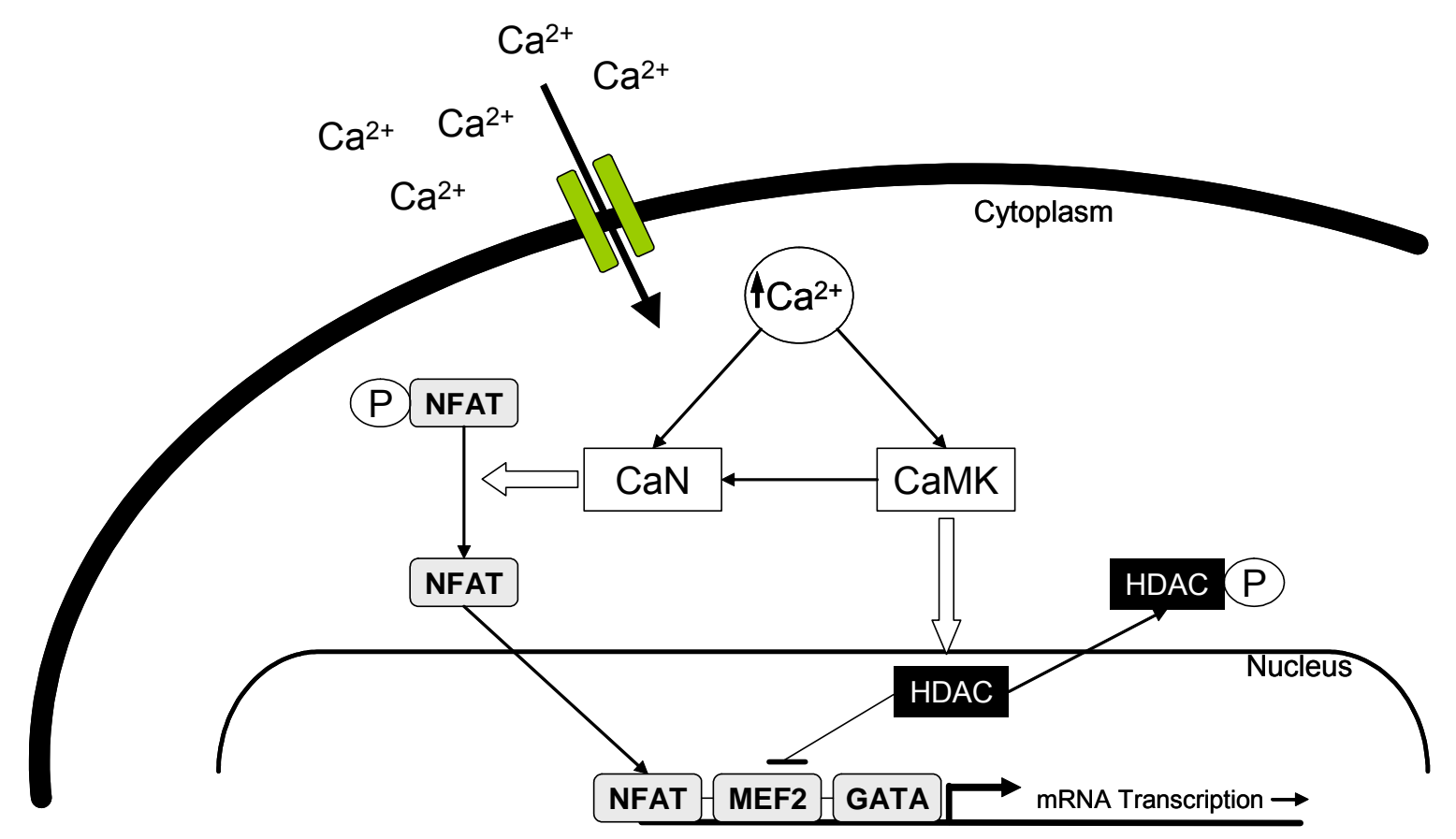

Figure 1.6 Proposed mechanisms for calcium-dependent increases in gene expression with skeletal muscle contractile activity. Bars denote inhibition and arrows denote activation. $\mathrm{Ca}^{2+}$, calcium; CaN, calcineurin; CaMK, calmodulin kinase; HDAC, histone deacetylase; MEF2, myocyte enhancer factor 2; NFAT, nuclear factor of activated T-cells (adapted from Liu et al. 2005).

Conclusive mechanistic data linking the effect of CaMK activation to cellular adaptation is currently lacking but downstream effects may be mediated, at least in part, through histone deacetylase nuclear extrusion via calcium signalling (Figure 1.6; Chin 2005; 
Liu et al. 2005). Prolonged, low-amplitude intra-cellular calcium transients increase calcineurin activity that dephosphorylates and activates transcriptional promoters to upregulate gene expression (Michel et al. 2004; Koulmann and Bigard 2006). The principle substrate targets of calcineurin include the nuclear factor of activated T cells (NFAT) and myocyte enhancer factor 2 (MEF2) families of transcription factors (Liu et al. 2005). Calcineurin has been implicated in several adaptive responses inducing muscle fibre growth / regeneration and slow fibre-type gene expression (Dunn et al. 1999; Musaro et al. 1999; Naya et al. 2000; Sakuma et al. 2003). Calcineurin appears to act as a co-regulator of muscle hypertrophy with insulin-like growth factor and may also contribute to myogenic proliferation and differentiation of satellite cells during skeletal muscle regeneration (Dunn et al. 1999; Musaro et al. 1999; Dunn et al. 2000; Sakuma et al. 2003; Scicchitano et al. 2005). The use of calcium / calmodulin inhibitors has been shown to suppress growth of overloaded muscle fibres while calcineurin over expression reduces disuse atrophy (Dunn et al. 1999; Dunn et al. 2000). In addition, research suggests calcineurin is also involved in fibre-type plasticity and fast-to-slow phenotype transformation (Naya et al. 2000; Michel et al. 2004; Parsons et al. 2004; Talmadge et al. 2004). Indeed, it has been proposed that calcineurin-induced gene expression of slow fibre and oxidative genes may result in the acquisition of a more metabolically efficient phenotype (Chin et al. 1998; Wu et al. 2000a; Michel et al. 2004). Such diverse calcineurin regulated adaptive pathways appear paradoxical (i.e. hypertrophy versus oxidative phenotype) but may represent alternating adaptation responses that are specific to the intensity and duration of contractile activity.

While research investigating $\mathrm{Ca}^{2+}$-calmodulin-calcineurin-dependent signalling has provided mechanistic insight into the role of this pathway in skeletal muscle, it should be noted our current understanding appears to be limited to cell culture and transgenic animal models. Importantly, few works have investigated the role of $\mathrm{Ca}^{2+}$-dependent signalling in 
humans, and the effect of exercise on calmodulin-calcineurin pathways remains to be established.

\section{Insulin / Insulin-like Growth Factor (IGF) Signalling Pathway}

The insulin / IGF signalling pathway incorporates many of the molecular components critical to our current understanding of muscle proteolysis and regulation of hypertrophy and atrophy processes (Glass 2003; Heszele and Price 2004; Sartorelli and Fulco 2004; Glass 2005; Taniguchi et al. 2006). Moreover, many of these components have additional roles for the regulation of glucose uptake, glycogen synthesis, and cell growth and differentiation (Taniguchi et al. 2006). The expression and importance of IGF-1 in skeletal muscle has been demonstrated in a variety of models from cell culture to human in vivo (DeVol et al. 1990; Vandenburgh et al. 1991; Adams and McCue 1998; Musaro et al. 1999; Chakravarthy et al. 2000b; Rommel et al. 2001; Dehoux et al. 2004; Stitt et al. 2004; Latres et al. 2005).

Contractile activity in skeletal muscle stimulates the secretion of IGF-1 which acts as an autocrine / paracrine growth factor, binding to its membrane receptor and initiating a cascade of molecular events (Figure 1.7; Glass 2003). IGF-1 binding to the receptor tyrosine kinase phosphorylates the insulin receptor substrate-1 (IRS-1) which subsequently activates phosphatidylinositol-3-OH kinase (PI3K; Backer et al. 1992). PI3K catalyses phosphate transfer (phosphatidylinositol-4, 5 biphosphate $\left[\mathrm{PIP}_{2}\right] /-3,4,5$ triphosphate $\left[\mathrm{PIP}_{3}\right]$ ) which ultimately recruits additional kinases, protein kinase $\mathrm{B} / \mathrm{AKT}$ and PDK1 (3' phosphoinositide-dependent protein kinase 1) to the cell membrane (Stephens et al. 1998; Wick et al. 2000). AKT may be negatively regulated via PIP3 dephosphorylation by lipidbinding phosphatase and tensin homologue (PTEN) and SH2-containing inositol 5'phosphatase-2 (SHIP2; Sleeman et al. 2005; Tang et al. 2005). Nevertheless, when activated AKT phosphorylates multiple substrates mediating important aspects of carbohydrate metabolism as well as protein synthesis and degradation. 


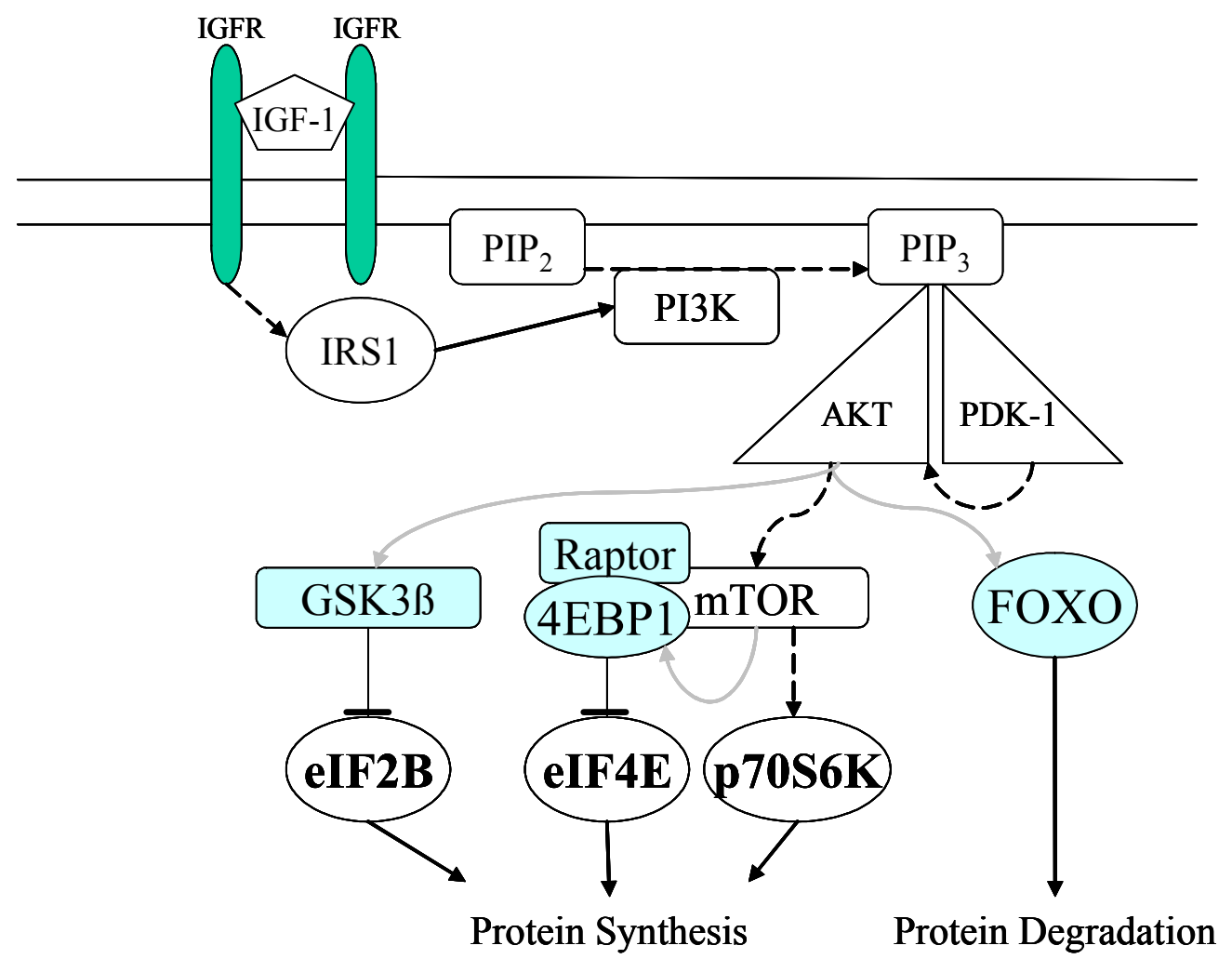

Figure 1.7 Simplified IGF-1 signalling pathway from receptor binding to protein synthesis.

Dashed arrows designate that phosphorylation activates the substrate and bars denote inhibition, whereas grey arrows denote phosphorylation events that result in inactivation of the substrate. IGFR, insulin-like growth factor receptor; IGF-1, insulin-like growth factor-1; IRS1, insulin receptor substrate 1; $\mathrm{PIP}_{2 / 3}$, phosphatidylinositol-4, 5 biphosphate / $-3,4,5$ triphosphate; PI3K, phosphatidylinositol-3-OH kinase; PDK1, 3' phosphoinositide-dependent protein kinase 1; FoxO, forkhead box O; mTOR; mammalian target of rapamycin; GSK3 $\beta$, glycogen synthase kinase $3 \beta$; 4EBP1, eukaryotic initiation factor 4E-binding protein; eIF2B/4E eukaryotic initiation factor 2B/4E; p70S6K, p70 ribosomal S6 kinase (adapted from Sartorelli and Fulco 2004).

\section{AKT / Protein Kinase B}

There are three isoforms of the serine / threonine AKT family, two of which (AKT1 and 2) are primarily expressed in skeletal muscle (Glass 2003; Nader 2005). Furthermore, the 
different AKT isoforms appear to have distinct functions: AKT1 has been associated with muscle hypertrophy whereas AKT2 has been implicated in signalling to glucose transport (Taniguchi et al. 2006). Following translocation to the cell membrane AKT is phosphorylated by PDK1 at threonine ${ }^{308}$ but requires secondary phosphorylation at serine ${ }^{473}$ for full activation, a role for which the mammalian target of rapamycin-rictor (mTOR-rictor) complex has recently been implicated (Sarbassov et al. 2005b; Taniguchi et al. 2006). Of late, a PH domain leucine-rich repeat protein phosphatase (PHLPP) has been shown to dephosphorylate $\mathrm{AKT}^{\mathrm{ser} 473}$ and suppress growth in cancer cells but this down-regulation of AKT has yet to be confirmed in skeletal muscle (Gao et al. 2005).

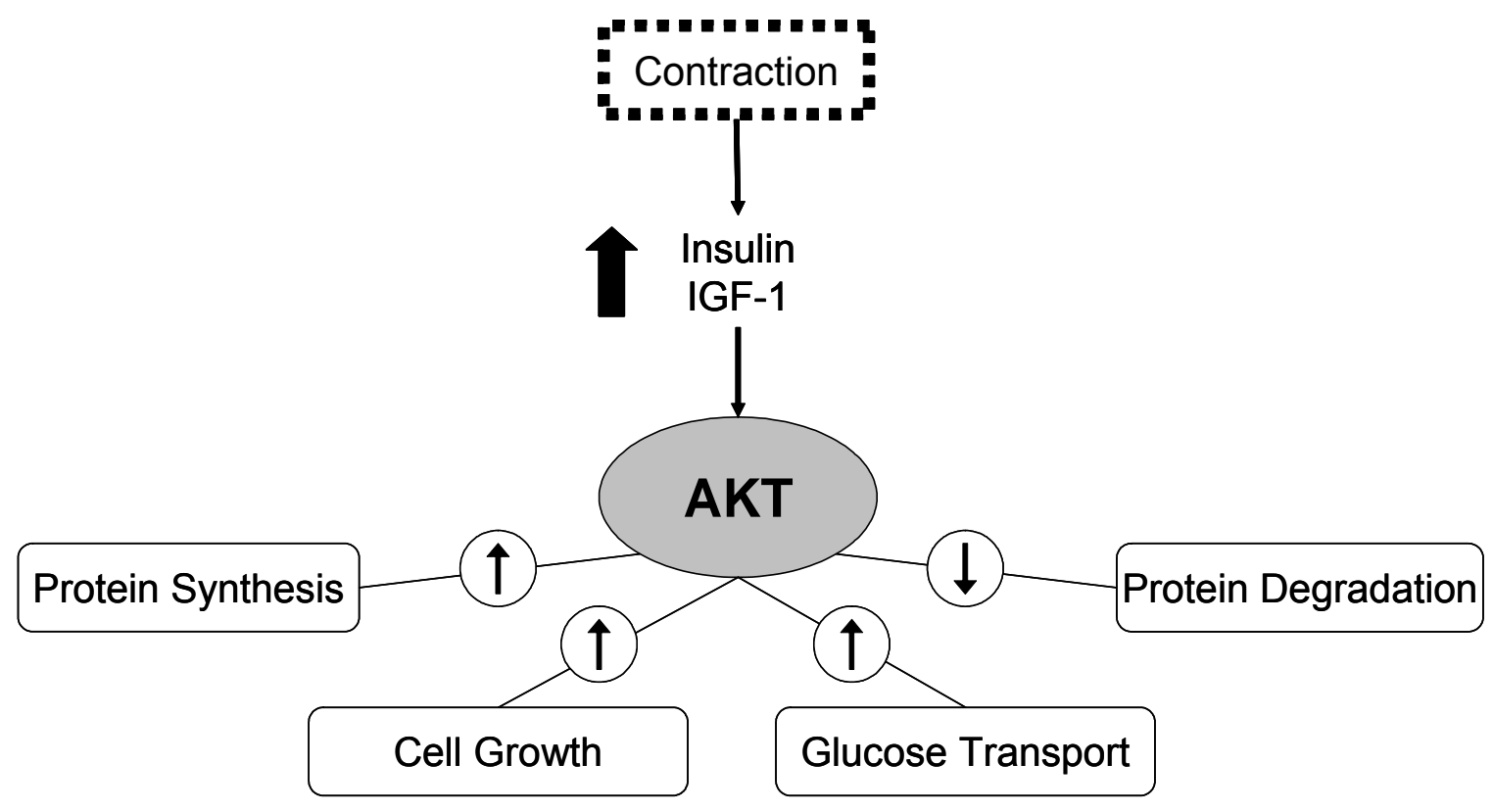

Figure 1.8 Proposed adaptive events associated with activation of AKT in skeletal muscle.

IGF-1, insulin-like growth factor-1.

AKT has numerous molecular targets commensurate with its varied physiological functions including those involved in protein synthesis (mTOR, tuberous sclerosis complex 2 [TSC2], glycogen synthase kinase 3 [GSK3]), atrophy (forkhead box O1 [FoxO1]), and glucose uptake (AKT substrate of $160 \mathrm{kDa}$ [AS160]) (Figure 1.8; Bodine et al. 2001b; Rommel et al. 2001; Inoki et al. 2002; Stitt et al. 2004; Bruss et al. 2005; Cai et al. 2006). 
While mTOR is considered a down-stream target of AKT via phosphorylation at serine $\mathrm{e}^{2448}$ (Nave et al. 1999; Bodine et al. 2001b), evidence is emerging that mTOR also phosphorylates AKT when bound to its associated protein rictor suggesting mutual activation under certain conditions (Hresko and Mueckler 2005; Sarbassov et al. 2005b; Sarbassov et al. 2006). Regardless, there is strong evidence the PI3K-AKT-mTOR pathway mediates hypertrophy in skeletal muscle via activation of translation initiation and increased ribosomal protein content (Bodine et al. 2001b; Rommel et al. 2001; Lai et al. 2004; Nader et al. 2005). In addition, AKT regulation of AMPK activity and direct phosphorylation of TSC2 and GSK3 $\beta$ may suppress their role as inhibitors of protein synthesis (Rommel et al. 2001; Inoki et al. 2002; Hahn-Windgassen et al. 2005; Cai et al. 2006). AMPK phosphorylates TSC2 which subsequently inhibits the hypertrophic actions of mTOR, while GSK3 $\beta$ prevents activation of eukaryotic initiation factor (eIF) 2B (Tee et al. 2002; Vyas et al. 2002; Garami et al. 2003; Hahn-Windgassen et al. 2005). Thus, in addition to $\mathrm{mTOR}^{\mathrm{ser} 2448}$ phosphorylation, AKT may also indirectly enhance translation initiation and protein synthesis through inhibition of AMPK-TSC2 and GSK $\beta$. Similarly, phosphorylation of the nuclear transcription factor FoxO by PI3K-AKT has been shown to sequester FoxO from the nucleus to the cytosol preventing transcription initiation of atrophy genes responsible for degradation of contractile protein, thereby mediating a protective effect on skeletal muscle by down-regulating pathways of protein degradation (Rena et al. 2002; Stitt et al. 2004; Latres et al. 2005).

There is conflicting data regarding AKT responses to contractile activity in skeletal muscle (Table 1.1). A variety of electrical stimulation and surgical ablation protocols have been shown to increase AKT phosphorylation in both red and white rodent skeletal muscle (Turinsky and Damrau-Abney 1999; Nader and Esser 2001; Sakamoto et al. 2002; Atherton et al. 2005; Bruss et al. 2005; Thomson and Gordon 2006). 
Table 1.1 Summary of research investigating AKT responses to contractile overload in skeletal muscle.

\begin{tabular}{|c|c|c|c|}
\hline Author & Species & Stimulus & Results \\
\hline Thomson \& Gordon (2006) & Rat & One wk surgical ablation & $\mathbf{P}+\mathbf{T}->100 \%$ increase \\
\hline Atherton et al. (2005) & Rat & 1) HFES - 10 x6@3 s contractions 2) LFES 3 h & $\mathbf{P}-1) \mathrm{HFES}=8$-fold increase 2) LFES = no change \\
\hline Parkington et al. (2003) & Rat & HFES - $10 \times 10,3 \mathrm{~s}$ contractions & $\mathbf{P}+\mathbf{T}-$ no change \\
\hline Sakamoto et al. (2002) & Rat & In situ contractions $(1,2.5,5,15,30 \mathrm{~min})$ & $\mathbf{P}$ - increased in all muscle types (range 1.6-13-fold) \\
\hline & & 3) Treadmill-30min @83\% $\mathrm{VO}_{2 \max }$ & 3) Run = no change \\
\hline Turinsky/Damrau-Abney (1999) & Rat & ES of the calf muscle ( 1 contraction $/ \mathrm{s} 5,15,25 \mathrm{~min}$ ) & AKT 1-A - 3-fold increase, AKT 2+3-A - no effect \\
\hline Sherwood et al (1999) & Rat & ES of the sciatic nerve $(2,5,10,15,30,60 \mathrm{~min})$ & A - no change \\
\hline Lund et al. (1998) & Rat & In vitro contractions of soleus ( $5 \mathrm{~min})$ & A - no change \\
\hline
\end{tabular}

$\mathbf{P}$, phosphorylation; A, activity; T, total protein; ES, electrical stimulation; HF, high-frequency; LF, low-frequency. 
Table 1.2 Summary of research investigating AKT responses to exercise in skeletal muscle.

\begin{tabular}{|c|c|c|c|}
\hline Author & Species & Stimulus & Results \\
\hline Dreyer et al. (2006) & Human & Resistance $10 \times 10$ leg extensions @ 70\% 1RM & $\mathbf{P}-100 \%$ increase \\
\hline Eliasson (2006) & Human & Resistance 1) 4 × 6 ECC 2) 4 x 6 CON & $\mathbf{P}$ - no change \\
\hline Wilson et al. (2006) & Human & Cycle 60 min @ $70 \% \mathrm{VO}_{2 \max }$ & $\mathbf{P}-50 \%$ increase \\
\hline Creer et al. (2005) & Human & Resistance-3 x $10 @ 70 \% 1 \mathrm{RM}$ & $\mathbf{P}-1.5$-fold increase \\
\hline Sakamoto et al. (2004) & Human & $\begin{array}{l}\text { Cycle 1)30min@75\% } \mathrm{VO}_{2 \max } \text { 2)6x60 s@125\% } \\
\mathrm{VO}_{2 \max }\end{array}$ & $\begin{array}{l}\mathrm{A}-\text { increase 1) } 75 \% \mathrm{VO}_{2 \max }=40 \% \text { 2) } 125 \% \mathrm{VO}_{2 \max } \\
=110 \%\end{array}$ \\
\hline Thorell et al. (1999) & Human & Cycle-60 min@70\% $\mathrm{VO}_{2 \max }$ & $\mathbf{P}-180 \%$ increase \\
\hline Widegren et al. (1998) & Human & Cycle-60 min one-leg@70\% $\mathrm{VO}_{2 \max }$ & A - no change \\
\hline Williamson et al. (2006) & Rat & Treadmill $-10 \%$ grade $26 / \mathrm{m} / \mathrm{min}$ for $10,20,30 \mathrm{~min}$ & $\mathbf{P}$ - no change \\
\hline Reynolds et al. (2004) & Rat & Voluntary wheel running (3 months) & $\mathbf{P}+\mathbf{T}-45-50 \%$ increase \\
\hline Krisan et al. (2004) & Rat & Resistance-3x10@75\% 1RM 3/wk (12wk) & $\mathbf{A}+\mathbf{T}-$ no change \\
\hline Bolster et al. (2003) & Rat & Resistance $-1 \times 50$ each day for $4 \mathrm{~d}$ & $\mathbf{P}-\sim 200 \%$ increase \\
\hline Sakamoto et al. (2003) & Rat & $\begin{array}{l}\text { Treadmill 1) } 60 \mathrm{~min} @ 20 \mathrm{~m} / \mathrm{min}+12 \% \text { grade } 2) \\
16 \mathrm{~m} / \mathrm{min} \text {, grade increased } 1 \% \text { every } 2 \mathrm{~min} \text { to fatigue }\end{array}$ & $\begin{array}{r}\mathbf{A}-\text { increase 1) } 60 \mathrm{~min}=80 \%-150 \% \\
\text { 2) Fatigue }=150 \%-150 \%\end{array}$ \\
\hline Markuns et al. (1999) & Rat & Treadmill-5, 10,30,60 min @ 25m/min (10\% gde) & $\mathbf{A}+\mathbf{P}=$ no change \\
\hline Wojtaszewski et al (1999) & $\begin{array}{c}\text { Mice } \\
(\mathrm{MIRKO})\end{array}$ & Treadmill-60min@22m/min (10\%) & $\mathbf{P}-$ no effect \\
\hline
\end{tabular}

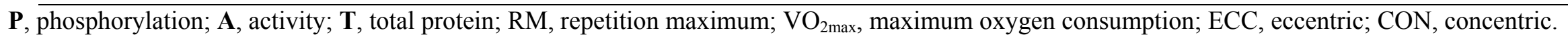


Equally, a number of studies have also observed no change in phosphorylation of AKT following electrical stimulation (Brozinick Jr. and Birnbaum 1998; Lund et al. 1998; Sherwood et al. 1999; Parkington et al. 2003). Moreover, in vivo contraction after running and resistance training in rodents has also produced conflicting findings where AKT has been reported to increase (Bolster et al. 2003b; Sakamoto et al. 2003; Reynolds et al. 2004) or remain unchanged (Markuns et al. 1999; Nader and Esser 2001; Krisan et al. 2004; Eliasson et al. 2006; Williamson et al. 2006b) in response to exercise (Table 1.2). Notably, Bolster et al. (2003b) examined the time-course of AKT response to resistance exercise and observed peak AKT phosphorylation 5-15 min following a bout of resistance training. Conversely, Dreyer and colleagues (2006) observed an increase in AKT phosphorylation 60 min after an acute bout of resistance training in humans. Differences in the contractile stimulus, timing of muscle tissue collection and isoform specific response may have contributed to the diverse and conflicting findings.

Work by Wilson et al. (2006), Sakamoto and colleagues (2004) and Thorell and coworkers (1999) has shown that moderate duration (30- $60 \mathrm{~min})$ low- $\left(\sim 70 \% \mathrm{VO}_{2 \max }\right)$ and highintensity $\left(\sim 125 \% \mathrm{VO}_{2 \max }\right)$ cycling exercise is sufficient to increase AKT phosphorylation in humans. Similarly, Creer et al. (2005) observed a significant increase in AKT phosphorylation $10 \mathrm{~min}$ after a bout of moderate-intensity resistance training. In contrast, Widegren and co-workers (1998) found no change in AKT activity 15 min post-exercise after 60 min cycling at $70 \% \mathrm{VO}_{2 \max }$. These conflicting results bring into question the specific role and functions of AKT in contraction-mediated training responses. However, differences in species, experimental design, fibre-type, substrate availability, and mode and intensity / duration of contractile activity may partly explain some of these discordant findings.

While strong evidence exists for AKT as a critical regulator of adaptation in skeletal muscle, defining and validating its role with in vivo exercise models remains elusive. Heavy resistance training increases muscle tension and hypertrophy while endurance training 
increases substrate turnover and utilisation. Therefore, the increase in AKT observed with diverse exercise modes described previously may be expected considering AKT's putative regulation of protein synthesis and glucose transport, respectively. Indeed, AKT appears to be a primary signalling mediator of training-specific adaptation to both endurance and resistance training in skeletal muscle.

\section{Mammalian target of rapamycin (mTOR)}

Protein complexes involving mTOR (also known as FKBP12 rapamycin-associated protein, FRAP) are capable of sensing diverse signals and produce a multitude of responses including mRNA translation, ribosomal biogenesis and nutrient metabolism (Sarbassov et al. 2005a). Two mTOR protein complexes exist where mTOR binds with a G-beta-L protein $(\mathrm{G} \beta \mathrm{L})$ and either a rapamycin-sensitive raptor or a rictor protein (Figure 1.9; Kim et al. 2003; Sarbassov et al. 2005b; Sarbassov et al. 2006). Work utilising rapamycin to manipulate mTOR activity indicates the mTOR-G $\beta$ L-raptor complex is a positive regulator of cell growth while mTORG $\beta$ L-rictor appears to have a key role in AKT activation and actin cytoskeleton regulation (Takano et al. 2001; Sarbassov et al. 2004; Park et al. 2005; Wang et al. 2005; Sarbassov et al. 2006).

Due to mTOR-rictor mediated activation of AKT it appears mTOR bound with rictor acts upstream and therefore is involved in regulating mTOR-raptor. In addition to AKT, upstream regulators of mTOR-raptor include the Ras homologue enriched in brain (Rheb) G protein (Manning and Cantley 2003). Rheb is in turn regulated upstream by the TSC1/2 complex and has been shown to regulate mTOR signalling and its subsequent downstream targets by an unknown mechanism (Tee et al. 2002; Garami et al. 2003). Primary downstream targets of mTOR-raptor include p70 ribosomal S6 kinase (p70 S6K), eukaryotic initiation factor 4E-binding protein (4E-BP1) and eukaryotic initiation factor 4B (eIF4B) which links mTOR with mRNA translation and increased protein synthesis and cell size (Bodine et al. 2001b; Raught et al. 2004; Ali and Sabatini 2005; Ohanna et al. 2005; Ruvinsky and Meyuhas 
2006). However, the mechanisms of mTOR-raptor activity in adaptation processes are not known and roles for additional kinases in this process cannot be ruled out (Sarbassov et al. 2005a). Nevertheless, altered mTOR activity has been observed in response to nutrients, mechanical stimuli and contraction in skeletal muscle (Hornberger et al. 2004; Parkington et al. 2004; Vary and Lynch 2006).

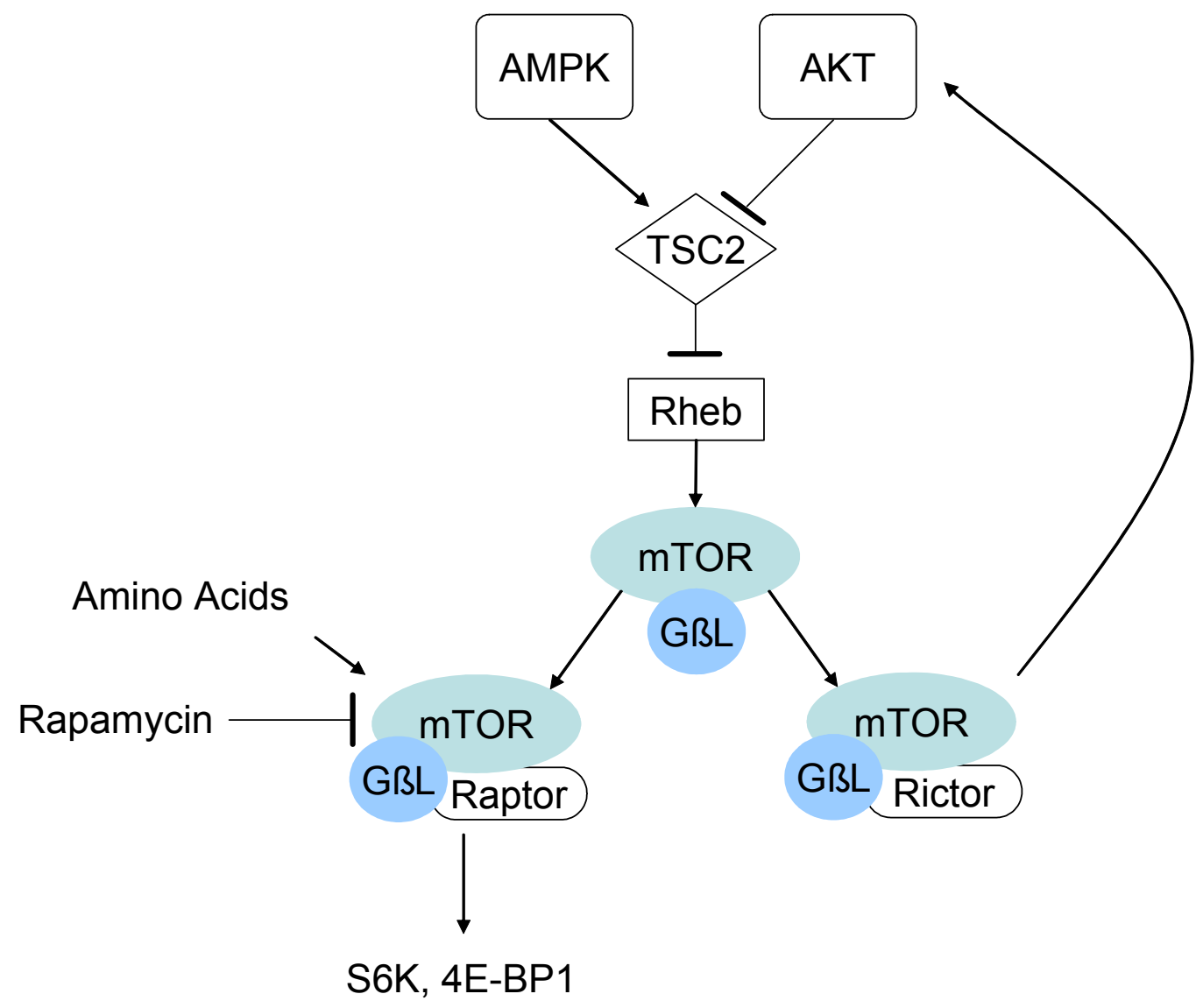

Figure 1.9 Model of the regulation and functions of the mammalian target of rapamycin (mTOR) complex. Bars denote inhibition and arrows denote activation. AMPK, adenosine monophosphate kinase; TSC2, tuberous sclerosis complex 2; Rheb, Ras homologue enriched in brain; GßL, G beta L protein; 4E-BP1, eukaryotic initiation factor 4E-binding protein; S6K, ribosomal S6 kinase; (adapted from Sabassov 2005).

Overloaded plantaris following surgical ablation has been shown to increase mTOR phosphorylation and total protein in both young and aged rodent skeletal muscle (Reynolds et 
al. 2002; Thomson and Gordon 2006). Work by Parkington and colleagues (Parkington et al. 2003; Parkington et al. 2004) and Atherton and co-workers (2005) has used intermittent highfrequency electrical stimulation and induced significant increases in mTOR phosphorylation in a number of different muscle groups. Conversely, no change in mTOR phosphorylation was seen following sustained low-frequency electrical stimulation, suggesting a tensionspecific contractile response (Atherton et al. 2005). However, these contractile stimuli may not represent physiological loading as voluntary wheel running and resistance training in rodents have been shown to increase mTOR phosphorylation (Bolster et al. 2003b; Reynolds et al. 2004).

It is difficult to reconcile an increase in mTOR activity following an endurance exercise stimulus such as voluntary wheel running, although emerging evidence suggests that mTOR may be involved in regulating mitochondrial function (Schieke et al. 2006). However, the reported changes in aged rodents following chronic (3 months) wheel running were increased total mTOR protein content but not mTOR activity (Reynolds et al. 2004). Accordingly, the elevated total mTOR protein in aged rodent muscle relative to sedentary control may be the result of exercise-induced maintenance of muscle mass with aging rather than stimulating translation initiation and protein synthesis. In contrast, increased mTOR activation following resistance training seems plausible given its proposed role in protein synthesis in response to a hypertrophy stimulus (Bolster et al. 2003b). Few studies have investigated the activation of mTOR in skeletal muscle of humans in vivo. Recently, Dreyer and co-workers (2006) have shown an increase in mTOR phosphorylation up to $2 \mathrm{~h}$ after resistance exercise in humans, providing strong evidence for the role of mTOR in anabolic processes following resistance training. Indeed, mTOR appears to be a primary regulator of protein synthesis in response to resistance training that may also be modulated by physical activity with aging. 
p70 S6K, 4E-BPI\& eIF2B

The most well-defined effectors of the PI3K-AKT-mTOR signalling pathway are the proteins implicated in translational control: ribosomal protein S6 kinase (S6K), eukaryotic initiation factor 4E-binding protein (4E-BP1) and eukaryotic initiation factor 2B (eIF2B; Bolster et al. 2003a; Ruvinsky and Meyuhas 2006). Mammalian cells express two S6K isoforms (S6K1 and 2) and S6K1 subsequently has duel cytosolic (p70 S6K) and nuclear (p85 S6K) isoforms (Ruvinsky \& Meyuhas 2006). S6K appears to function downstream of mTOR-raptor and rodent knockout models have revealed that of the two isoforms, S6K1 predominantly regulates cell size in skeletal muscle (Shima et al. 1998; Ohanna et al. 2005). mTOR-raptor also regulates and suppresses 4E-BP1 (also known as phosphorylated heat and acid soluble protein stimulated by insulin, PHAS-1) via hyper-phosphorylation to derepress 4E-BP1 inhibition of translation initiation cap binding protein eIF4E (Richter and Sonenberg 2005; Sarbassov et al. 2005a). Alternately, GSK3 regulates eIF2B and inhibits its actions which initiate ribosomal translation (Pavitt 2005). GSK3 is a phosphorylation target of AKT, which inhibits its negative effect on eIF2B thereby increasing translation and protein synthesis, and is therefore controlled to a large extent via insulin / IGF signalling (Welsh et al. 1997; Jefferson et al. 1999).

S6K exerts its effect through multiple substrate targets and has been implicated in orchestrating the regulation of numerous cellular functions (Figure 1.10; Ruvinsky and Meyuhas 2006). Numerous studies provide compelling support for the fundamental role of p70 S6K in skeletal muscle hypertrophy (Baar and Esser 1999; Bodine et al. 2001b; Nader and Esser 2001; Karlsson et al. 2004; Lai et al. 2004; Atherton et al. 2005). Early work by Baar and Esser (1999) established a strong association between increased p70 S6K activity and skeletal muscle hypertrophy following $6 \mathrm{wk}$ of high-frequency electrical stimulation. Subsequently, work from Bodine and co-workers (2001b) used surgical ablation and pharmacological intervention to highlight the important role for p70 S6K in skeletal muscle 
hypertrophy processes. Further studies reveal that the exercise-induced p70 S6K response occurs with a resistance training-like stimulus and that endurance exercise does not upregulate p70 S6K activity (Nader and Esser 2001; Atherton et al. 2005; Kubica et al. 2005). Indeed, recent work showing increased p70 S6K phosphorylation with stretch activated mechanotransduction in skeletal muscle suggests that eccentric loading may be critical for S6K1 activation and therefore hypertrophy (Hornberger et al. 2005a; Hornberger et al. 2005b; Spangenburg and McBride 2006).

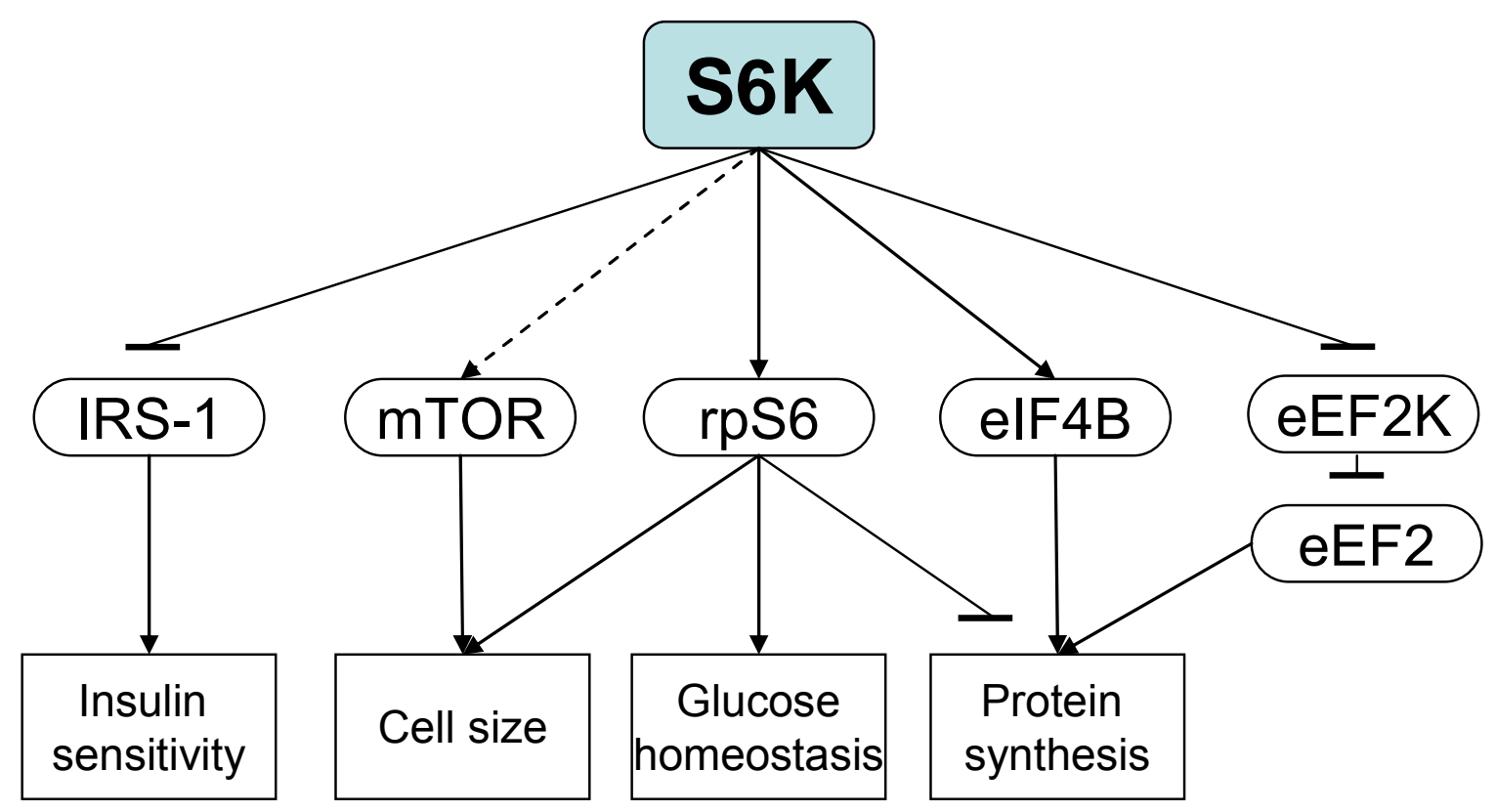

Figure 1.10 Putative downstream targets and functions of ribosomal protein S6K. Bars denote inhibition, arrows denote activation, and dashed lines indicate a putative effect. IRS-1, insulin receptor substrate-1; mTOR; mammalian target of rapamycin; eIF4B, eukaryotic initiation factor 4B; S6K, ribosomal protein S6 kinase; rpS6, ribosomal protein S6; eEF2, eukaryotic elongation factor 2; eEF2K, eukaryotic elongation factor 2 kinase. (adapted from Ruvinsky and Meyuhas 2006). 
Studies performed on humans support the results of investigations undertaken in rodents in which up-regulation of S6K1 and p70 S6K has been observed following an acute bout of resistance training (Karlsson et al. 2004; Eliasson et al. 2006; Koopman et al. 2006). The chronic regulation of hypertrophy and other cell processes by S6K1 is less clear considering its possible reciprocal effects on protein synthesis and negative feedback to PI3KAKT-mTOR signalling through IRS-1 phosphorylation (Ruvinsky and Meyuhas 2006).

Given the well-documented co-regulation by mTOR-raptor, it is not surprising that 4E-BP1 phosphorylation in skeletal muscle emulates that of S6K1 to a large extent. Studies utilising surgical ablation and high-frequency electrical stimulation consistently show an increase in 4E-BP1 phosphorylation in skeletal muscle in rodents (Bodine et al. 2001b; Atherton et al. 2005; Funai et al. 2006; Thomson and Gordon 2006). Furthermore, as translation initiation cap binding is prevented when dephosphorylated 4E-BP1 is bound to eukaryotic initiation factor (eIF) 4E, skeletal muscle overload has also been shown to produce a decrease in 4E-BP1-eIF4E binding and subsequent increases in eIF4E-eIF4G association (Bodine et al. 2001b; Kubica et al. 2005). Significant increases in phosphorylation of 4E-BP1 have also been observed following several resistance exercise models (Bolster et al. 2003a; Kubica et al. 2005). Conversely, an endurance-like stimulus has been shown to decrease 4EBP1 phosphorylation indicating a negative effect of endurance training on translational machinery and protein synthesis (Atherton et al. 2005). Interestingly, Koopman and colleagues (2006) recently observed reduced 4E-BP1 phosphorylation immediately and $2 \mathrm{~h}$ after an acute bout of resistance training. However, these authors incorporated an atypical resistance training protocol including a high number of contractions with short recovery periods generating elevated metabolic stress and AMPK activity. This training stimulus could reduce activation of pathways involved in protein synthesis in a manner similar to endurance training. 
Contraction-induced eIF2B activity is predominantly controlled by AKT-GSK3 $\beta$ and represents a rate limiting step for translation initiation (Welsh et al. 1997; Jefferson et al. 1999; Pavitt 2005). Moreover, evidence exists that eIF2B may also be regulated in an mTOR dependent manner (Kubica et al. 2005). Nevertheless, of the multiple eIF2B subunits the epsilon subunit may represent a principal site for activation with contraction (Kubica et al. 2005). Contractile activity has been shown to reduce GSK3 $\beta$ phosphorylation of eIF2B thereby relieving inhibition and increasing translation (Farrell et al. 2000; Kostyak et al. 2001; Vary et al. 2002). In response to resistance exercise significant dephosphorylation of eIF2B enhancing its availability for translation initiation and increases in eIF2B total protein and mRNA levels have been shown (Farrell et al. 2000; Kostyak et al. 2001; Kubica et al. 2004; Kubica et al. 2005). The effect of endurance training on eIF2B is unclear although in view of its regulation of glycogen metabolism, GSK3 activity would be expected to increase with endurance exercise but may be repressed during recovery with carbohydrate feeding. Indeed, Atherton and colleagues (Atherton et al. 2005) employed endurance-like contractile activity in rodent skeletal muscle and observed a significant increase in GSK3 $\beta$ and eIF2B (deactivation) phosphorylation immediately and following $3 \mathrm{~h}$ recovery, respectively. However, eIF2B responses to resistance or endurance exercise have not been investigated in human skeletal muscle.

Taken together, these findings provide strong evidence for the resistance traininginduced increase in protein synthesis via IGF signalling, but may also include other currently unknown kinases acting directly on mTOR-raptor or its downstream kinases. In addition, endurance training appears to have a significant negative effect on the translational machinery. 


\section{Cytokine Signalling}

Cytokines function as inter-cellular signalling molecules and act as facilitators of receptor subunit assembly to induce an intra-cellular signal (Cannon and St. Pierre 1998). Cytokines are small polypeptides released at the site of inflammation in response to numerous factors including cachexia, sepsis, and exercise-induced muscle damage (Cannon and St. Pierre 1998; Jackman and Kandarian 2004; Glass 2005; Petersen and Pedersen 2005). Several cytokines have been implicated in initiating protein degradation and suppression of protein synthesis following injury in skeletal muscle, most notably tumor necrosis factor alpha (GarciaMartinez et al. 1993; Glass 2005; Williamson et al. 2005).

\section{Tumor Necrosis Factor Alpha (TNFa)}

Elevated TNF $\alpha$ concentration generates an increase in the activity of ubiquitin-conjugated protein degradation and inhibition of insulin / IGF mediated protein synthesis (GarciaMartinez et al. 1993; Garcia-Martinez et al. 1994; Fernandez-Celemin et al. 2002; Plomgaard et al. 2005; Williamson et al. 2005; Lang et al. 2006). Furthermore, TNF $\alpha$ has also been shown to negatively affect anabolic processes by destabilising myogenic differentiation and altering transcriptional activity (Langen et al. 2004; Vashisht Gopal et al. 2006). Suppression of the insulin / IGF signalling pathway by TNF $\alpha$ has been shown to induce insulin resistance via impaired phosphorylation of IRS-1 and the AKT substrate AS160 kDa (del Aguila et al. 1999; de Alvaro et al. 2004; Plomgaard et al. 2005), and suppress protein synthesis through a decrease in IGF-1 and IGF-binding protein gene expression, subsequently inhibiting elongation initiation and mRNA translational efficiency in skeletal muscle (Lang et al. 2002; Williamson et al. 2005; Lang et al. 2006). Early studies using intravenous administration of TNF $\alpha$ revealed a significant time-dependent increase in free ubiquitin and ubiquitin gene expression highlighting the role of TNF $\alpha$ with regard to elevated muscle proteolysis (GarciaMartinez et al. 1993; Garcia-Martinez et al. 1994). This effect has subsequently been linked to downstream intermediates stimulating ubiquitin ligase gene expression and therefore muscle 
atrophy (Fernandez-Celemin et al. 2002; Li et al. 2003; Cai et al. 2004; Li et al. 2005; Lang et al. 2006).

Work by Sriwijitkamol and colleagues (2006) revealed a $40 \%$ decrease in TNF $\alpha$ protein content in subjects following eight wk of cycling, indicating a positive effect of lowintensity aerobic exercise on low-grade inflammation and metabolic status in skeletal muscle. Conversely, strenuous exercise, particularly heavy eccentric contractions associated with resistance training, would be expected to generate an acute increase in local inflammation and rise in $\mathrm{TNF} \alpha$ concentration. Indeed, there is a significant increase in circulating systemic $\mathrm{TNF} \alpha$ after muscle damaging eccentric resistance training and marathon running (Ostrowski et al. 1999; Del Aguila et al. 2000). Similarly, Hamada and colleagues (2004) observed an increase in TNF $\alpha$ mRNA abundance $3 \mathrm{~d}$ after a 45 min bout of downhill running in muscle samples of healthy subjects, indicative of an acute inflammatory response associated with exercise-induced muscle damage. Thus, it appears that exercise is capable of generating proand anti-inflammatory effects on skeletal muscle in an intensity- and mode-dependent manner, mediated at least in part via TNF $\alpha$ activation. However, the chronic effects of repeated bouts of high-intensity exercise and the specific adaptive response with altered intensity, duration and frequency of contraction and mode of exercise on TNF $\alpha$ activity remain to be established.

Previous studies have predominantly utilised cell culture, pathological states or intravenous administration to identify the effect of TNF $\alpha$. Hence, our knowledge of contraction-induced changes in TNF $\alpha$ and subsequent alterations within skeletal muscle is limited. Moreover, most investigations have focused on regulators and effectors of TNF $\alpha$ signalling, namely inhibitor of NFkB kinase (IKK), nuclear factor kappa enhancer binding protein $(\mathrm{NF \kappa B})$ and p38 mitogen activated protein kinase. 
Inhibitor Kappa B Kinase (IKK) \& Nuclear Factor Kappa B (NFאB)

The binding of $\mathrm{TNF} \alpha$ to its receptor at the cell membrane initiates a cascade of intra-cellular events. An important component of this cytokine mediated pathway is the activation of a kinase that inhibits I $\mathrm{KB}$ (Chen 2005; Chen et al. 2006). When I $\mathrm{B}$ B is bound to the nuclear transcription factor $\mathrm{NF \kappa B}$, I $\mathrm{B}$ B suppresses its role as a promoter of gene expression (Chen 2005; Natoli et al. 2005; Tergaonkar et al. 2005; Bosisio et al. 2006). TNFa signals through the TNF-receptor-associated factor (TRAF) and transforming growth factor beta activated kinase 1 (TAK1) proteins to phosphorylate and activate IKK (Chen et al. 2006).

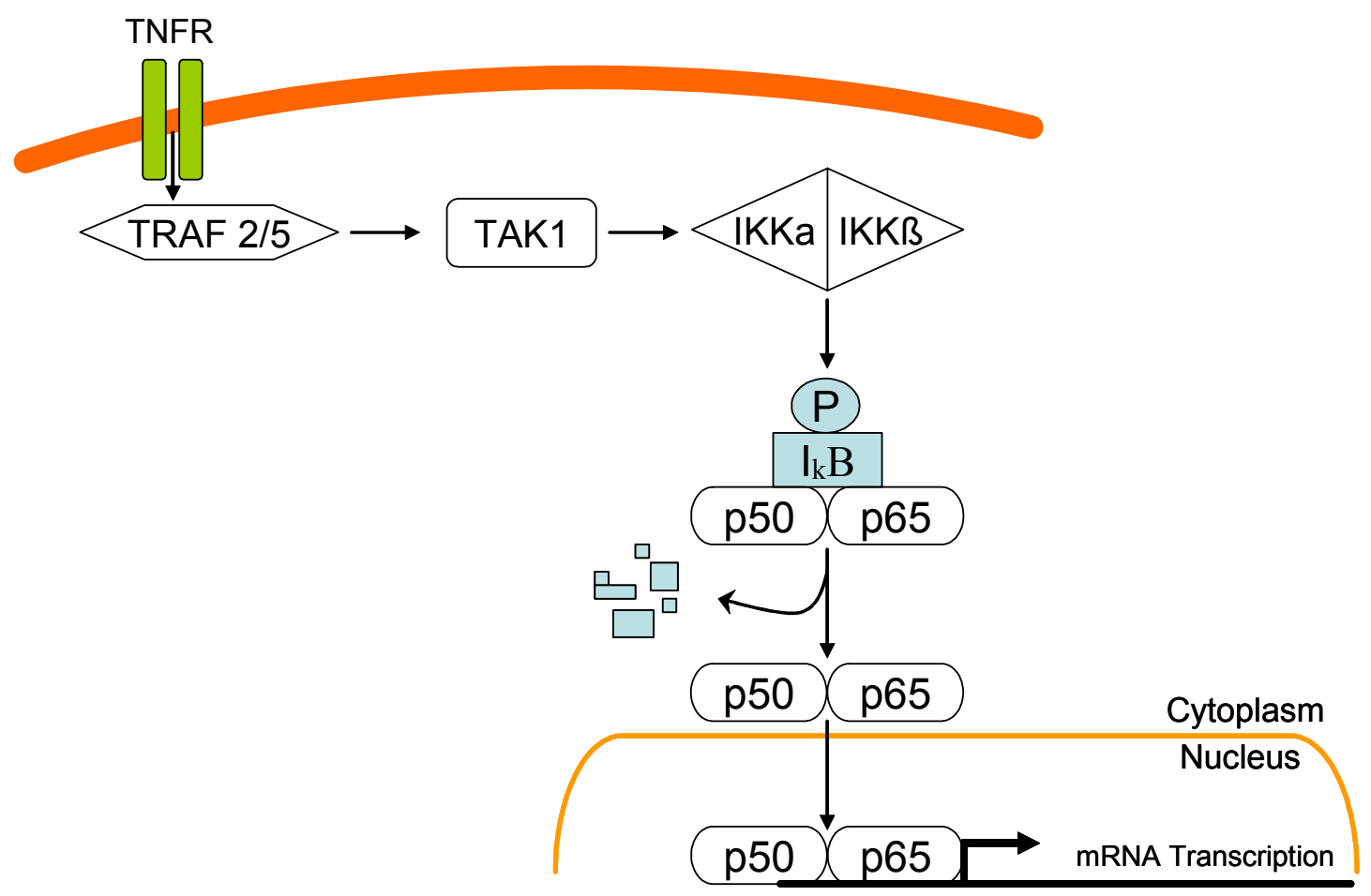

Figure 1.11 Simplified TNF $\alpha$ pathway initiating nuclear transcription. TNFR, tumor necrosis factor receptor; TRAF2/5, TNF-receptor-associated factor 2/5; TAK1, transforming growth factor beta activated kinase $1 ; \mathrm{I} \kappa \mathrm{B}$, inhibitor of nuclear factor kappa enhancer binding protein; IKK $\alpha / \beta$, inhibitor kappa $\mathrm{B}$ kinase; $\mathrm{P}$, phosphorylation; $\mathrm{p} 50 / 65$, nuclear factor kappa enhancer binding protein complex (adapted from Chen $2005 \&$ Chen et al. 2006). 
Phosphorylation of I $\mathrm{I} B$ by IKK targets it for ubiquitination and subsequent degradation, releasing the $\mathrm{NF \kappa B}$ dimeric complex (p50/p65) to initiate the expression of genes involved in multiple cell processes including ubiquitin-mediated protein degradation (Figure 1.11; Alkalay et al. 1995; Chen et al. 1995; Scherer et al. 1995; Tergaonkar et al. 2005). Moreover, while this orthodox regulation of NFkB activity is well-accepted there are numerous additional putative mechanisms of activation and transcriptional and posttranslational control (Bae et al. 2003; Duran et al. 2003; Schwabe and Sakurai 2005; Steinbrecher et al. 2005; Kearns et al. 2006). Indeed, NFאB exists as multiple complexes resulting in altered function and is induced by a diverse array of stimuli with immense diversity in the effects and consequence of its activation that can both promote and suppress cell growth and metabolic status (Perkins and Gilmore 2006).

Recently, work by Cai and colleagues (2004) established a mechanistic link between IKK $\beta$ / NF $\mathrm{B}$ and expression of atrophy genes in skeletal muscle confirming the findings of previous investigations that identified a relationship between NFKB activation and muscle atrophy in models of disuse and muscular dystrophy (Hunter et al. 2002; Kumar and Boriek 2003). Cai and co-workers (2004) utilised muscle-specific transgenic expression of IKK $\beta$ or an $\mathrm{I} \kappa \mathrm{B}$ repressor to selectively activate or repress $\mathrm{NF \kappa B}$ and skeletal muscle wasting, and the subsequent gene expression of the atrophy promoting ubiquitin ligase muscle ring finger protein (MuRF). Additional work in cell culture provides support for the proposed initiation of ubiquitin-proteasome transcriptional machinery in a NFkB-dependent manner (Wyke and Tisdale 2005). Collectively, these results indicate that up-regulation of the IKK / NFkB / MuRF pathway exacerbates atrophy in skeletal muscle.

Given the capacity of contractile activity to induce local and systemic stress / inflammation and disturb cell homeostasis, exercise represents a stimulus capable of increasing NFkB activity in skeletal muscle. Yet, contractile activity is also of critical importance for maintaining cell functions including enhanced protein synthesis and 
preserving muscle mass. Ex vivo mechanical stretch of diaphragm muscle activates IKK /

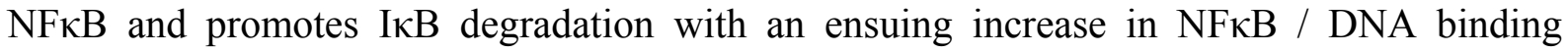
(Kumar and Boriek 2003). Likewise, an acute bout of endurance exercise (60 min, 75\% $\mathrm{VO}_{2 \max }$ ) has been shown to decrease $\mathrm{I} \kappa \mathrm{B}$ content, and increase IKK and $\mathrm{NF} \kappa \mathrm{B}$ phosphorylation in rodent skeletal muscle (Ji et al. 2004; Ho et al. 2005). In contrast, Durham and colleagues (2004) observed a decrease in $\mathrm{NF \kappa B}$ activity following a severe bout of resistance training in humans and with electrical stimulation in rodents. While the low sample numbers (four human, five rodent) necessitate that these results are interpreted cautiously, an explanation for these discordant findings is unclear. Indeed, a vigorous resistance training session with repeated eccentric contractions would typically be expected to result in muscle damage, inflammation and muscle remodelling. To date, only one training study has investigated the effects of exercise on TNF $\alpha$ signalling. In that investigation, 14 subjects performed 8 wk of sub-maximal cycling ( $4 \mathrm{x}$ wk, $45 \mathrm{~min}, 70 \% \mathrm{VO}_{2 \text { peak }}$ ) and had increased $\mathrm{I} \kappa \mathrm{B}$ and reduced $\mathrm{TNF} \alpha$ protein content in skeletal muscle, indicating a decrease in IKK / NFKB signalling (Sriwijitkamol et al. 2006). These findings may be interpreted as a protective effect of sub-maximal intensity aerobic activity on the inflammatory response, decreasing local inflammation and cellular disturbance.

Altered transcriptional responses through signal transduction to NFkB with exerciseinduced contraction may be an important process in skeletal muscle adaptation. Acute training bouts may increase NFKB activity for transient adaptive events such as immune or antiapoptotic responses, while chronic training may reduce total $\mathrm{NF} \kappa \mathrm{B}$ activity and subsequent protein degradation enabling net protein synthesis. Regardless, due to the innumerable functional outcomes of $\mathrm{NF \kappa B}$ transcriptional control much work remains to fully elucidate the cause and effect relationship between exercise and NFkB activity. 


\section{Mitogen Activated Protein Kinase (MAPK) Signalling Pathway}

Of all the secondary messenger systems, the mitogen activated protein kinase pathway may be the most complex, induced by a variety of stimuli and regulating numerous diverse functions in mammalian cells. The MAPK family is conserved in eukaryotic cells and co-ordinately regulates activities including gene expression, mitosis, metabolism, survival and apoptosis (Figure 1.12; Roux and Blenis 2004). Of the five distinct MAPK groups, two have been most

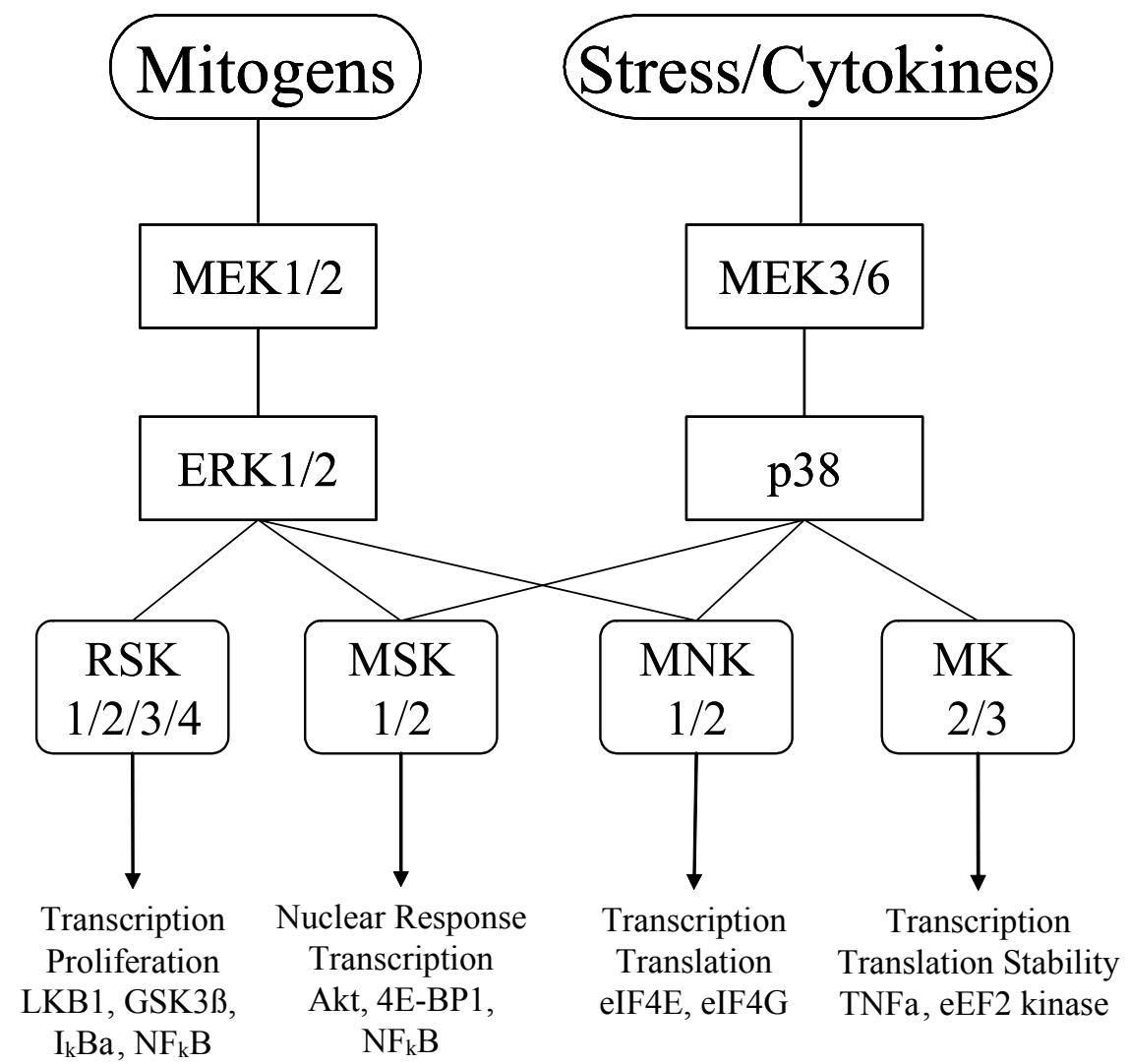

Figure 1.12 Proposed pathways of activation and molecular targets of MAPK signalling. MAPK, mitogen activated protein kinase; MEK, mitogen activated kinase; ERK, extracellular regulated kinase; RSK, ribosomal S6 kinase; MSK, mitogen and stress activated kinase; MNK, MAPK-interacting kinase; MK, mitogen protein kinase; GSK, glycogen synthase kinase; IкB, inhibitor kappa $\mathrm{B}$; $\mathrm{NF} \kappa \mathrm{B}$, nuclear factor kappa $\mathrm{B}$; $\mathrm{TNF} \alpha$, tumor necrosis factor alpha; eEF, eukaryotic elongation factor; eIF, eukaryotic initiation factor; 4E-BP1, eukaryotic initiation factor 4E-binding protein (adapted from Roux and Blenis 2004). 
characterised with regard to exercise responses, the extracellular signal-regulated kinases 1 and $2($ ERK1/2) and p38 isoforms $\alpha, \beta, \gamma$, and $\delta$ (Sakamoto \& Goodyear 2002). ERK1/2 appear to be preferentially activated in response to growth factors while p38 is more responsive to an assortment of stress stimuli (Roux and Blenis 2004).

Upon activation ERK1/2 and p38 can modulate gene expression and protein synthesis via phosphorylation of multiple targets including cytosolic proteins and transcription factors, or translocate directly to the nucleus where they can also regulate transcription factor activity (Figure 1.12; Sakamoto \& Goodyear 2002). Moreover, the capacity of exercise-induced contractile activity to induce MAPK activation is undeniable yet determining the specificity of response and the resultant physiological outcome remains elusive (Nielsen et al. 2003a; Thong et al. 2003; Williamson et al. 2003).

\section{ERK1/2}

A relatively large number of studies have investigated the effect of exercise on MAPK activity in vivo human skeletal muscle (Table 1.3) and increased ERK1/2 activity has been consistently observed following exercise regardless of intensity, duration, mode of exercise or subject training status. Interestingly, this response may be attenuated with aging (Williamson et al. 2003). It has been postulated that ERK1/2 is involved in a "general" response to exercise that could include up-regulation of non-specific immediate early response genes or signalling pathways important for increasing global rates of transcription and / or translation (Assoian 2002; Galetic et al. 2003; Atherton et al. 2005; Shahbazian et al. 2006; Williamson et al. 2006b). Despite the common response of ERK1/2 with diverse exercise stimuli it is reasonable to suggest that ERK1/2 mediates divergent adaptation processes. Moreover, the role of ERK1/2 in regulating immediate early gene (IEG) responses and the cell cycle programme has been shown to be dependent on the strength and duration of the signal (Wu et al. 2000b; Assoian 2002; Murphy et al. 2002; Murphy et al. 2004). Signal duration of ERK1/2 
Table 1.3 Summary of research investigating MAPK responses to exercise in vivo human skeletal muscle.

\begin{tabular}{|c|c|c|}
\hline Author & Stimulus & Results \\
\hline Creer et al. (2005) & Resistance 3 x $10 @ 70 \%$ 1-RM 1) H-CHO 2) L-CHO & ERK-P 1) 120\% 2) 120\% p90 RSK-P 1) 290\% 2) 550\% \\
\hline \multirow[t]{2}{*}{ Richter et al. (2004) } & Cycle 1)10 min@35,60,85\% $\mathrm{VO}_{2 \max }$ & ERK-A 1) 100\%@35\% no further increase @ 60/85\% 2) 100\% \\
\hline & 2) $30 \min @ 35 \% \mathrm{VO}_{2 \max }$ & ERK-P 1) $35 \% \mathrm{VO} 2=\sim 100 \% ; 60 \% \mathrm{VO} 2=\sim 220 \% ; 85 \% \mathrm{VO} 2=\sim 500 \%$ 2) $\sim 100 \%$ \\
\hline McGee \& Hargreaves (2004) & Cycle60min@70\% VO 2 peak & Total p38-P 380\% Nuclear p38-P 80\% \\
\hline Chan et al. (2004) & Cycle 60 min @70\% VO 2peak 1) Normal 2) L-CHO & Nuclear p38-P 1) 80\% 2) 110\% \\
\hline Karlsson et al. (2004) & Resistance 4 x $10 @ 80 \%$ 1-RM 1) BCAA 2)water & ERK-P - 700\% p38-P - 400\% (no effect with BCAA) \\
\hline Nielsen et al. (2003) & Cycle20min@80\% $\mathrm{VO}_{2 \text { peak }}$ 1) Sed 2) Trained & ERK-A 1) $40 \%$ 2) $38 \%$ p38-P 1) $1000 \%$ 2) $950 \%$ \\
\hline Thompson et al. (2003) & 1) 50 eccentric contractions 2) $30 \mathrm{~min}-10 \%$ downhill treadmill & ERK-P 1) $200 \%$ 2) -53\% ERK-T 1) $424 \%$ 2) no change \\
\hline Thong et al. (2003) & 60 min 1-leg kicks (alt 5 min @ 75 \& 100\% PWC) & p38-P up $40 \%$ compared with rest leg \\
\hline Williamson et al. (2003) & Resistance 3 x $10(\mathrm{KE}) @ 70 \%$ 1-RM 1) old 2) young & ERK-P 1) -48\% 2) 75\% p90-P 1) -60\% 2) 100\% p38-P 1)-17\% 2) 18\% \\
\hline Yu et al. (2003) & Cycle 8 x 5 min @85\% VO 2peak 1) Untrained 2) Trained & ERK-P 1) $171 \%$ 2) 42\% p38-P 1) 120\% 2) $50 \%$ \\
\hline Yu et al. (2001) & Marathon & ERK-P 400\% p38 400\% \\
\hline Boppart et al. (2000) & Marathon & p38 $\gamma-\mathbf{P} 300 \%$ p38 $\gamma-\mathbf{A} 50 \%$ \\
\hline Krook et al. (2000) & Cycle1-leg@70\% VO $\mathrm{Vmax}_{\max }$ & MSK $1 / 2-A ~ 400 \%$ \& $200 \%$ p90 RSK-A 2500\% MapKap2-A 330\% vs. rest \\
\hline Widegren et al. (1998) & Cycle 1-leg@70\% $\mathrm{VO}_{2 \max }$ & ERK-P 31 fold @30 min (75\%30 min response @ 60 min) p38-P 2.2 fold \\
\hline Aronson et al. (1997) & Cycle60min@70\% $\mathrm{VO}_{2 \max }$ & ERK-P 4-24 fold \\
\hline
\end{tabular}

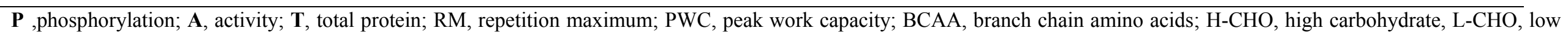
carbohydrate; KE, knee extension. 
alters the functional activity of gene product and ultimately controls the biological outcome (Murphy et al. 2002). Indeed, in regulating progression of the myogenic programme ERK1/2 appears to initially interfere with differentiation but later enhances myotube formation (Wu et al. 2000b). In addition, a high degree of crosstalk may also contribute to regulation of ERK1/2 activity. Notably, AKT has been shown to regulate components of ERK1/2 signalling, finetuning differentiation and proliferation in the cell cycle (Rommel et al. 1999; Moelling et al. 2002; Galetic et al. 2003).

Distinguishing between sustained and transient signal duration and alterations in signal strength may significantly alter the role of ERK1/2 in subsequent adaptation processes, particularly in satellite cell activation. Collectively, these factors may represent a mechanism for the specificity of response with diverse modes of exercise considering the extended duration of contraction with endurance training versus the greater contraction intensity during heavy resistance training. Evidence exists for a specific role of ERK1/2 in response to resistance training, in which ERK1/2 has been implicated as a regulator of hypertrophy in skeletal muscle. Phosphorylation of TSC2 by ERK1/2 suppresses TSC2 inhibition of mTOR and downstream mediators of translation initiation (Ma et al. 2005). ERK1/2 also directly regulates eIF4B phosphorylation via its downstream effector p90 ribosomal protein S6 kinase (RSK) increasing translation efficiency (Shahbazian et al. 2006). Regardless, due to the common response of ERK1/2 following endurance and resistance exercise the precise functions of ERK1/2 have yet to be determined and more work is needed to clearly define the role of ERK1/2 in specific exercise-induced adaptation.

p38

Activation of p38 MAPK has been observed following both endurance and resistance exercise in humans (Yu et al. 2001; Karlsson et al. 2004; McGee and Hargreaves 2004). It seems that p38 is responsible for the regulation of multiple cell processes including myogenic and mitochondrial gene expression and ubiquitin ligase activity making it difficult to establish a 
clear role for p38 in the adaptation response to exercise (Akimoto et al. 2005; Li et al. 2005; Lluis et al. 2006). Previous findings have established an unequivocal role for p38 MAPK in controlling muscle gene expression in the myogenic process (Wu et al. 2000b; Lluis et al. 2006). This multi-step process that is primarily orchestrated by myogenic regulatory factors is in large part regulated by p38 which may also act as a molecular switch for the activation of satellite cells (Suelves et al. 2004; Jones et al. 2005; Lluis et al. 2005). In addition, a study by Li and colleagues (2005) revealed that p38 was also involved in the regulation of muscle atrophy, mediating the $\mathrm{TNF} \alpha$-induced gene expression of muscle atrophy F-box protein. Therefore, p38 may be involved in the regulation of both hypertrophy and atrophy processes suggesting an important role for p38 in adaptation responses to resistance training. The complexity of MAPK-mediated signalling in skeletal muscle is highlighted by the notion that p38 has recently been implicated in mitochondrial biogenesis (Akimoto et al. 2005). Akimoto and co-workers (2005) initially observed a correlation between the peroxisome proliferator activated receptor $\gamma$ co-activator $1 \alpha(\mathrm{PGC}-1 \alpha)$, a transcription factor that promotes mitochondrial biogenesis, and p38 MAPK activation. In addition, work in cells and transgenic mice by these workers revealed PGC- $1 \alpha$ and cytochrome oxidase IV (COX IV) protein expression occurred in response to p38 activation. Furthermore, p38 activity has also been shown to regulate myocyte enhancer factor 2 (MEF2) dependent genes, including PGC-1 $\alpha$ (Zhao et al. 1999; McGee and Hargreaves 2004). Thus, p38 may promote mitochondrial biogenesis and therefore may also have a significant role in adaptation following endurance training. The conundrum of MAPK responses to exercise limits our current understanding of the potential functions and physiological outcomes that contribute to the specificity of training adaptation. Indeed, much of our knowledge regarding MAPK responses with exercise rests on correlation data with little information currently available regarding the mechanisms of regulation. 


\section{Summary}

It is apparent that multiple secondary messengers and signalling pathways are activated or repressed in response to an exercise stimulus and that these pathways induce a multi-faceted adaptation process. While our current understanding of intra-cellular signalling is incompletely resolved, it is clear that exercise-induced contractile activity generates activation of common and distinct signalling intermediaries in response to the intensity, duration and mode of contraction. Ultimately, this complex transduction network produces an increase in the transcription of particular genes and subsequent gene product to promote phenotypic adaptation in an exercise specific manner.

\subsection{Genetic and Molecular Responses to Exercise}

Adaptive events distal to primary and secondary signalling culminate in the synthesis of new protein containing genetically determined information to carry out specific functions (Bouchard et al. 1997; Flück and Hoppeler 2003). This molecular process is highly regulated at numerous sites from DNA copying to the assembly of gene product (Figure 1.13). While there is a paucity of evidence showing detailed continuity between specific signalling pathways, gene expression and subsequent protein synthesis, signalling proteins have been shown to activate numerous immediate early genes, transcription factors and molecular machinery responsible for promoting the rate of transcription of target gene messenger RNA (Zhao et al. 1999; Cai et al. 2004; Murphy et al. 2004; Sandri et al. 2004; Simone et al. 2004). Generally, the directional change of mRNA transcription is the same as the directional change of the protein generating a new steady state adaptation in the muscle milieu (Booth and Baldwin 1996). Increased mRNA quantity should translate into greater functional protein abundance although multiple control mechanisms may alter the end state adaptation. 


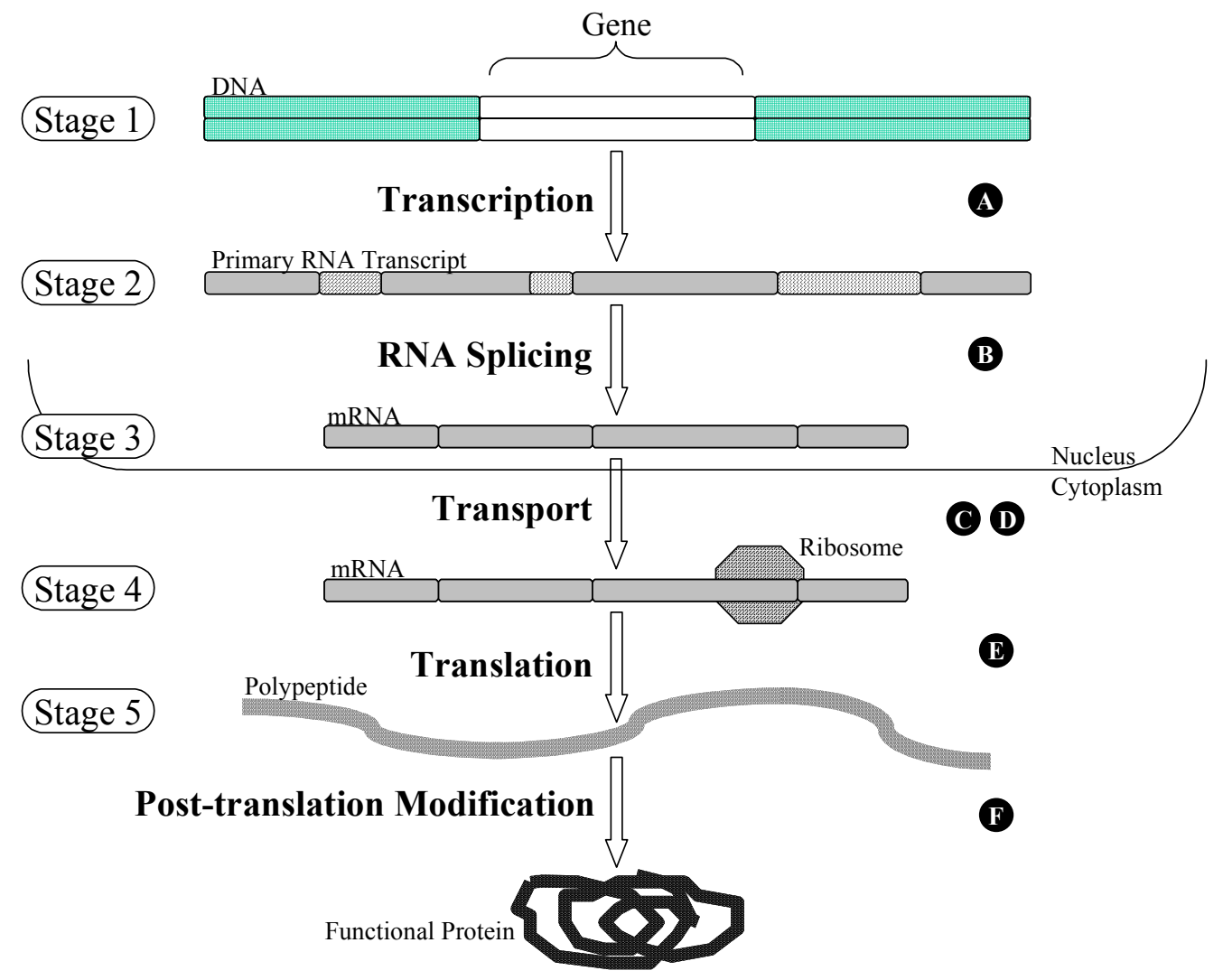

Figure 1.13 Five stages of gene expression and sites of regulation (A-F; adapted from Bouchard, Melina and Perusse 1997).

Moreover, the balance of mRNA synthesis versus degradation, the baseline abundance of each specific protein, and additional translational control mechanisms all contribute to net protein synthesis (Flück and Hoppeler 2003). The following sections will highlight important genetic and molecular adaptation responses with endurance and resistance exercise, and examine the effect of concurrent endurance and resistance training on the specificity of adaptation in skeletal muscle.

\subsubsection{Endurance Exercise}

Endurance training elicits both central and peripheral adaptations, alters neural recruitment patterns, and causes profound changes in muscle bioenergetics and enhanced morphological, metabolic substrate and acid-base status in skeletal muscle (Hawley 2002). Briefly, endurance 
adaptation results in increased muscle glycogen stores and glycogen sparing at submaximal workloads via increased fat oxidation, enhanced lactate kinetics and morphological alterations including greater type I fibre proportions per muscle area, and increased capillary and mitochondrial density. Moreover, repeated bouts of endurance exercise results in altered expression of a multiplicity of gene products, resulting in an altered muscle phenotype with improved resistance to fatigue (Adhihetty et al. 2003). Mitochondria are the main subcellular structures that determine the oxidative capacity and fatigue resistance to prolonged contractile activity in skeletal muscle (Hoppeler and Flück 2003). Endurance training can increase steady state mitochondrial protein content $50-100 \%$ within $\sim 6 \mathrm{wk}$, but a protein turnover half-life of $\sim 1$ wk means a continuous training stimulus is required to maintain elevated mitochondrial content (Hood 2001). While enhanced oxygen kinetics, substrate transport, and buffering capacity all contribute to enhanced endurance capability in skeletal muscle, improved endurance is most associated with the increase in mitochondrial density and enzyme activity, termed mitochondrial biogenesis (Adhihetty et al. 2003; Irrcher et al. 2003).

\section{Mitochondrial Biogenesis}

The expansion of the mitochondrial reticulum in skeletal muscle is a highly regulated and complex process that appears to require the co-ordinated expression of a large number of genes (Goffart and Wiesner 2003; Irrcher et al. 2003). Mitochondrial biogenesis requires the co-expression of both the nuclear and mitochondrial genomes for assembly and expansion of the reticulum and $95 \%$ of the genes necessary for biogenesis are encoded in the nucleus (Hood 2001; Goffart and Wiesner 2003). Thus, an important aspect of mitochondrial biogenesis is the import machinery regulating the transport of nuclear encoded precursor proteins into the organelle (Irrcher et al. 2003). However, expression of genes promoting mitochondrial biogenesis is predominantly controlled by the global principles of gene regulation, that is, transcription initiation and interaction at the gene promoter (Goffart and 
Wiesner 2003). Therefore, transcription factors and transcriptional co-activators represent critical regulators of mitochondrial biogenesis.

Numerous transcription factors have been implicated in regulating expression of genes involved in mitochondrial biogenesis (Adhihetty et al. 2003). While no single transcription factor has been found to be responsible for the co-ordination of mitochondrial gene expression, several candidates appear to be important for mitochondrial biogenesis. These include the immediate early genes (c-fos, c-jun), early growth response gene-1 (Egr-1) and nuclear respiratory factors-1 and -2 (NRF-1/2; Hood et al. 2006). Egr-1 is associated with promoting transcription of the electron transport chain protein cytochrome $\mathrm{C}$ oxidase (COX; (Freyssenet et al. 2004) while NRF-1 and NRF-2 are implicated in the transcriptional control of multiple mitochondrial genes including mitochondrial transcription factor A (Tfam) and recently identified mitochondrial transcription specificity factors TFB1M and TFB2M (Scarpulla 2002; Gleyzer et al. 2005). The Egr and NRF transcription factors increase in response to serum stimulation or contractile activity (Connor et al. 2001; Irrcher and Hood 2004; Gleyzer et al. 2005) and NRF-1 and -2 mRNA have been shown to increase in response to both acute and chronic endurance exercise (Baar et al. 2002; Short et al. 2003).

The peroxisome proliferator receptor gamma co-activator-1 alpha (PGC-1 $\alpha$ ) has been established as an important regulator of mitochondria content in skeletal muscle due to its apparent co-activation of multiple mitochondrial transcription factors (Finck and Kelly 2006; Hood et al. 2006). Indeed, PGC-1 $\alpha$ is the founding member of a family of transcriptional coactivators that has been proposed as a potential "master regulator" of mitochondrial biogenesis (Adhihetty et al. 2003; Scarpulla 2006). In support of this contention, Lin and coworkers (2002) over expressed PGC-1 $\alpha$ in mice skeletal muscle and observed increased proportions of type I fibres and increased resistance to fatigue. In addition, the biogenesis and maintenance of mitochondrial architecture is controlled by altered rates of mitochondrial protein fusion and fission (Santel and Fuller 2001), a role for which mitofusin (Mfn) 1/2 
proteins have been strongly implicated (Bach et al. 2003; Santel et al. 2003). Recent evidence indicates that PGC-1 $\alpha$ mediates a regulatory pathway involving estrogen-related receptor alpha $(\mathrm{ERR} \alpha)$ and $\mathrm{Mfn} 1 / 2$, and this pathway has been shown to be up-regulated following a 10-km cycling time trial (Cartoni et al. 2005; Soriano et al. 2006). This suggests that a PGC$1 \alpha$ activated pathway promotes an increase in mitochondrial content in response to endurance exercise through enhanced mitochondrial protein fusion.

Similarly, PGC-1 $\alpha$ also mediates Tfam activation, a key component in mitochondrial DNA replication and transcription (Kanki et al. 2004; Maniura-Weber et al. 2004). The NRF1 transcription factor has been shown to activate Tfam which enhances the capacity for assembly of protein complexes within the mitochondria (Scarpulla 2006). Therefore, as a coactivator of NRF-1 transcription PGC-1 $\alpha$ is involved in regulating Tfam function (Wu et al. 1999). Importantly, Tfam activity appears to increase in response to contractile activity and exercise suggesting enhanced mitochondrial protein assembly with endurance training (Bengtsson et al. 2001; Gordon et al. 2001).

Most notably, PGC-1 $\alpha$ is the co-activator of the peroxisome proliferator activated receptor (PPAR) family (Vega et al. 2000; Oberkofler et al. 2002). The three PPAR sub-types $\alpha, \gamma$ and $\delta$ have distinct functions but all appear to regulate lipid homeostasis via expression of genes involved in mitochondrial fatty acid oxidation (Lee et al. 2003; Finck and Kelly 2006). The physiological significance of increased PGC-1 $\alpha$-PPAR activated gene expression with endurance training is an enhanced capacity for fat utilisation during prolonged exercise, and may also be related to fast-to-slow fibre type conversion (Luquet et al. 2003; Wang et al. 2004). Indeed, this was highlighted by Wang and colleagues (2004) who generated transgenic mice over expressing PPAR $\delta$ that resulted in a 2.3-fold increase in mitochondrial DNA content, significant type I fibre transformation and a $90 \%$ increase in running performance (Figure 1.14). The small numbers of studies investigating PPAR activation following exercise support these findings where both acute (Jorgensen et al. 2005; Russell et al. 2005) and 
chronic (Luquet et al. 2003; Mahoney et al. 2005; Fritz et al. 2006) endurance exercise induces PPAR transcription.

A
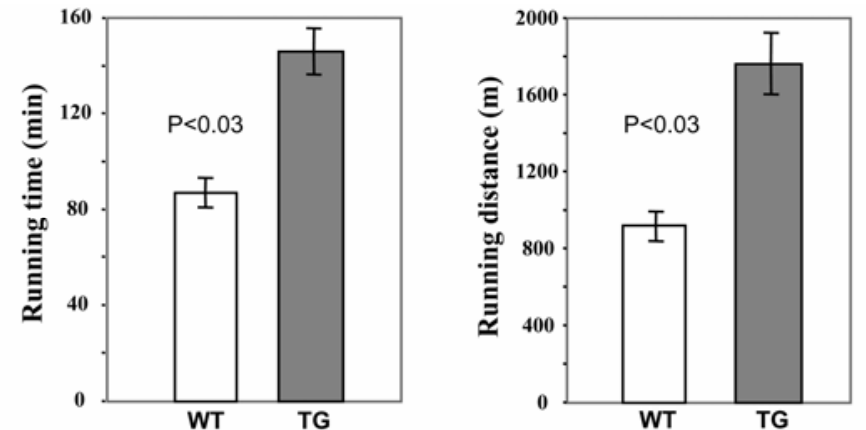

B
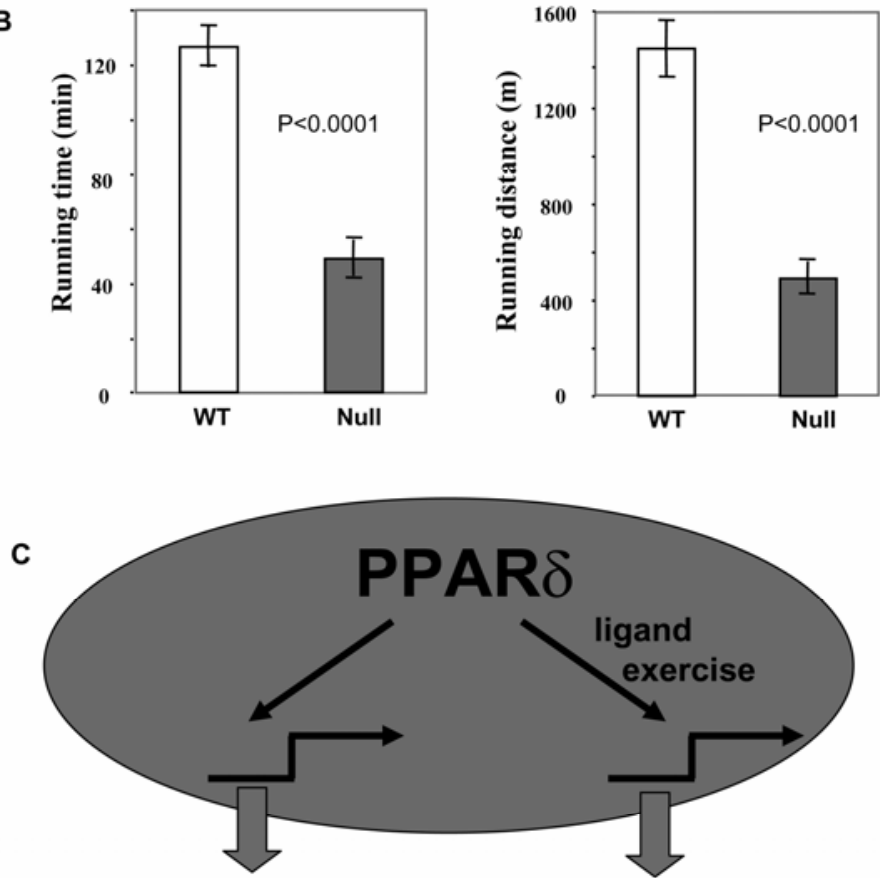

Oxidative metabolism

Mitochondrial biogenesis

Type I fiber formation

Exercise endurance

Figure 1.14 Peroxisome proliferator receptor delta (PPAR $\delta$ ) regulates exercise endurance. A) Enhanced exercise performance in the transgenic PPAR $\delta$-over expression mice (TG) versus wild type (WT) mice. B) Compromised exercise performance in transgenic PPAR $\delta$-null mice (Null) compared with wild type (WT) mice. C) Putative functions of PPAR $\delta$ in skeletal muscle (reproduced from Wang et al. 2004).

The available evidence suggests that increased PGC-1 $\alpha$-PPAR mediated transcription is an important endurance adaptation and imperative for establishing an endurance phenotype. 
Taken collectively, the results from the studies reviewed here not only implicate PGC-1 $\alpha$ in the regulation of aerobic metabolism but also mitochondrial architecture and fast-to-slow fibre type transformation. The upstream signalling events that activate PGC-1 $\alpha$ gene expression are not well defined and various pathways have been implicated including AMPK, calcineurin, p38 MAPK and FoxO1 (Zong et al. 2002; Akimoto et al. 2005; Southgate et al. 2005; Finck and Kelly 2006). Intriguingly, PGC-1 $\alpha$ activation may be complicated by the fact that some transcription factors that are co-activated by PGC-1 $\alpha$ may also be responsible for its upregulation (Hood et al. 2006).

Endurance exercise has been routinely shown as a potent stimulator of PGC-1 $\alpha$ gene and protein expression (Pilegaard et al. 2000; Baar et al. 2002; Norrbom et al. 2003; Psilander et al. 2003; Terada and Tabata 2003; Cluberton et al. 2005; Jorgensen et al. 2005; Terada et al. 2005). In contrast, due to its specific role in mitochondrial biogenesis, no studies have investigated the role of PGC-1 $\alpha$ following resistance training. However, as might be expected, a significant decrease in PGC-1 $\alpha$ protein was observed after high-frequency electrical stimulation to mimic the effects of resistance training (Atherton et al. 2005). Accordingly, exercise-induced up-regulation of PGC-1 $\alpha$ represents an essential molecular response in our current understanding of mitochondrial biogenesis and exercise specific adaptation to endurance but not resistance training in skeletal muscle.

\section{Metabolic Gene Expression}

In addition to mitochondrial biogenesis, increased gene expression of metabolic proteins following endurance exercise contributes to promoting an enhanced endurance phenotype (Mahoney et al. 2005). These include genes encoding enzymes and transporters involved in carbohydrate and fat metabolism such as hexokinase, lipoprotein lipase and carnitine palmitoyltransferase (Pilegaard et al. 2000; Tunstall et al. 2002). Endurance exercise has been shown to increase the mRNA abundance and transcription of a variety of metabolic genes in the post-exercise recovery period (Pilegaard et al. 2000; Tunstall et al. 2002; Hildebrandt et 
al. 2003; Pilegaard et al. 2005; Yang et al. 2005). This up-regulation of metabolic genes following exercise appears to peak in the initial hours of recovery and generally returns to resting levels within $24 \mathrm{~h}$ (Pilegaard et al. 2005; Yang et al. 2005). It has been postulated that the cumulative effect of this transient up-regulation with repeated bouts of exercise may be an underlying mechanism for exercise-induced adaptation with endurance training (Pilegaard et al. 2000). However, in contrast to PGC-1 $\alpha$ activity resistance training may also alter the expression of a number of metabolic genes (e.g. PDK4), but this moderate effect may be restricted to those proteins involved in carbohydrate metabolism (Yang et al. 2005). In addition, evidence suggests that nutrient availability has a significant effect on the exercise response of a variety of metabolic genes. Therefore, nutrient-gene interactions represent a variable that may promote or suppress metabolic exercise-induced adaptation in skeletal muscle (Vissing et al. 2004; Pilegaard et al. 2005; Hawley et al. 2006).

\subsubsection{Resistance Exercise}

Increased muscle cross-sectional area and altered neural recruitment patterns represent the principal adaptations to repeated bouts of heavy resistance exercise (Häkkinen 1989). Increased cross-sectional area (i.e. hypertrophy) following resistance training is a result of an increase in protein mass per cross-sectional area of tissue which occurs when the rate of protein synthesis is greater than protein degradation (Chesley et al. 1992; Phillips et al. 1997). Fundamentally, the hypertrophy response to overload is qualitatively and quantitatively controlled via the production of cellular proteins and new muscle cells. Adaptation to resistance training includes increased protein synthesis via regulatory changes in transcriptional and translational mechanisms, and in the production of muscle cells that are added to existing myofibres or combine and form new contractile filaments, each providing additional contractile machinery with which to generate force (Bolster et al. 2003a; Rennie et al. 2004; Sartorelli and Fulco 2004). In addition, while a degree of protein degradation is 
required for muscle remodelling resistance training may also decrease chronic activation of atrophy pathways resulting in supplementary net protein synthesis (Jones et al. 2004).

\section{Hypertrophy}

Compensatory hypertrophy in skeletal muscle following resistance training involves an increase in ribosomal protein synthesis (Bolster et al. 2003a). Regulation of ribosomal protein synthesis is controlled by phosphorylation events altering mRNA translation initiation, elongation and termination, and the cellular ribosome content which determines the synthesis of protein per mRNA (Farrell et al. 2000; Wang et al. 2001; Hannan et al. 2003; Richter and Sonenberg 2005). Modulation of translation initiation is a particularly important regulatory site for global protein synthesis in response to a resistance exercise stimulus and is the rate limiting step and most frequent target for translational control (Baar et al. 1999; Richter and Sonenberg 2005). The initiation step in translation is regulated by phosphorylation of eukaryotic initiation factors (eIFs) that mediate $40 \mathrm{~S}$ ribosomal subunit binding to mRNA (Bolster et al. 2003a). The binding of 40S ribosome-mRNA is mediated by the interaction of multiple eIFs and ribosomal S6 protein that are the most distal components of the insulin / IGF-1 and MAPK signalling cascades (Glass 2005; Kubica et al. 2005; Shahbazian et al. 2006). Given its ability to ultimately enhance protein synthesis through translation initiation IGF-1 and IGF-binding protein gene expression following an exercise stimulus has been the focus of extensive investigation.

Over expression and localised infusion of IGF-1 have both been shown to induce significant skeletal muscle hypertrophy (Adams and McCue 1998; Musaro et al. 2001; Song et al. 2005). While a number of IGF isoforms and splice variants exist there is growing evidence elucidating the potential mechanisms that directly promote IGF-1 induced hypertrophy. Musaro and co-workers (1999) have demonstrated that IGF-1 mediated protein synthesis and hypertrophy involves IGF-1 induced calcineurin activation of GATA-2 and nuclear factor of activated T-cell (NFAT) transcription factors generating an increase in gene 
expression. Moreover, there is compelling mechanistic data showing IGF-1 regulates increases in PI3K-AKT-mTOR signalling (Shen et al. 2005; Vary 2006). Importantly, these studies show p70 S6K and 4E-BP1 phosphorylation and eIF4E-eIF4G association, indicating IGF-1 activation of kinases proximal to the translational machinery. IGF-1 has also been shown to enhance satellite cell recruitment, proliferative potential and life span (Chakravarthy et al. 2000a; Chakravarthy et al. 2000b; Jacquemin et al. 2004). Thus, IGF-1 appears capable of inducing hypertrophy via an enhanced programme of gene expression, increased ribosomal-mediated translation, and satellite cell activation. Collectively, this strongly implicates IGF-1 as a potent multi-factorial regulator of hypertrophy. The co-ordination of the alternate adaptive machinery responsible for IGF-1 mediated hypertrophy responses is unclear but is likely to be specific to the intensity and duration of the overload stimulus.

Skeletal muscle IGF-1 and IGF-binding protein mRNA and protein content increases in response to contractile activity in a variety of overload models (Adams et al. 1999; Spangenburg et al. 2002; Hameed et al. 2003; Adams et al. 2004; Bickel et al. 2005). However, the effect of resistance exercise on IGF-1 in vivo in humans is equivocal, as IGF-1 mRNA has been reported to increase (Bamman et al. 2001; Kim et al. 2005; Petrella et al. 2006), decrease (Psilander et al. 2003; Bickel et al. 2005) and remain unchanged (Bickel et al. 2003; Hameed et al. 2003). Differences in exercise stimuli, individual variability and the unknown time course of expression for the IGF-1 response may provide some explanation for these discordant findings. Nonetheless, the IGF-1 genotype appears to enhance the strength response to resistance exercise in humans as the IGF-1 promoter polymorphism has been associated with greater strength gains following $10 \mathrm{wk}$ of resistance training (Kostek et al. 2005). Therefore, despite only partial clarity of in vivo human data the available evidence implicates IGF-1 gene expression in exercise-induced muscle hypertrophy in response to resistance training. 
Activation and differentiation of non-specialised satellite cells into new muscle cells is an additional mechanism that contributes to compensatory hypertrophy (Sartorelli and Fulco 2004). Eccentric contraction during resistance exercise is capable of inducing substantial damage to contractile and structural components of skeletal muscle (McCully and Faulkner 1985; MacPherson et al. 1997). Satellite cells located at the basal lamina that surrounds a myofibre are activated for the repair and maintenance of the muscle milieu and addition of myonuclei, both important components for muscle regeneration and hypertrophy (Zammit et al. 2004; Petrella et al. 2006). During the course of regeneration satellite cells first exit their quiescent state and proliferate until secondary molecular signals arrest the mitotic activity and initiate the differentiation of non-specialised cells to muscle precursor cells that migrate to the site of regeneration (Figure 1.15; Charge and Rudnicki 2004).

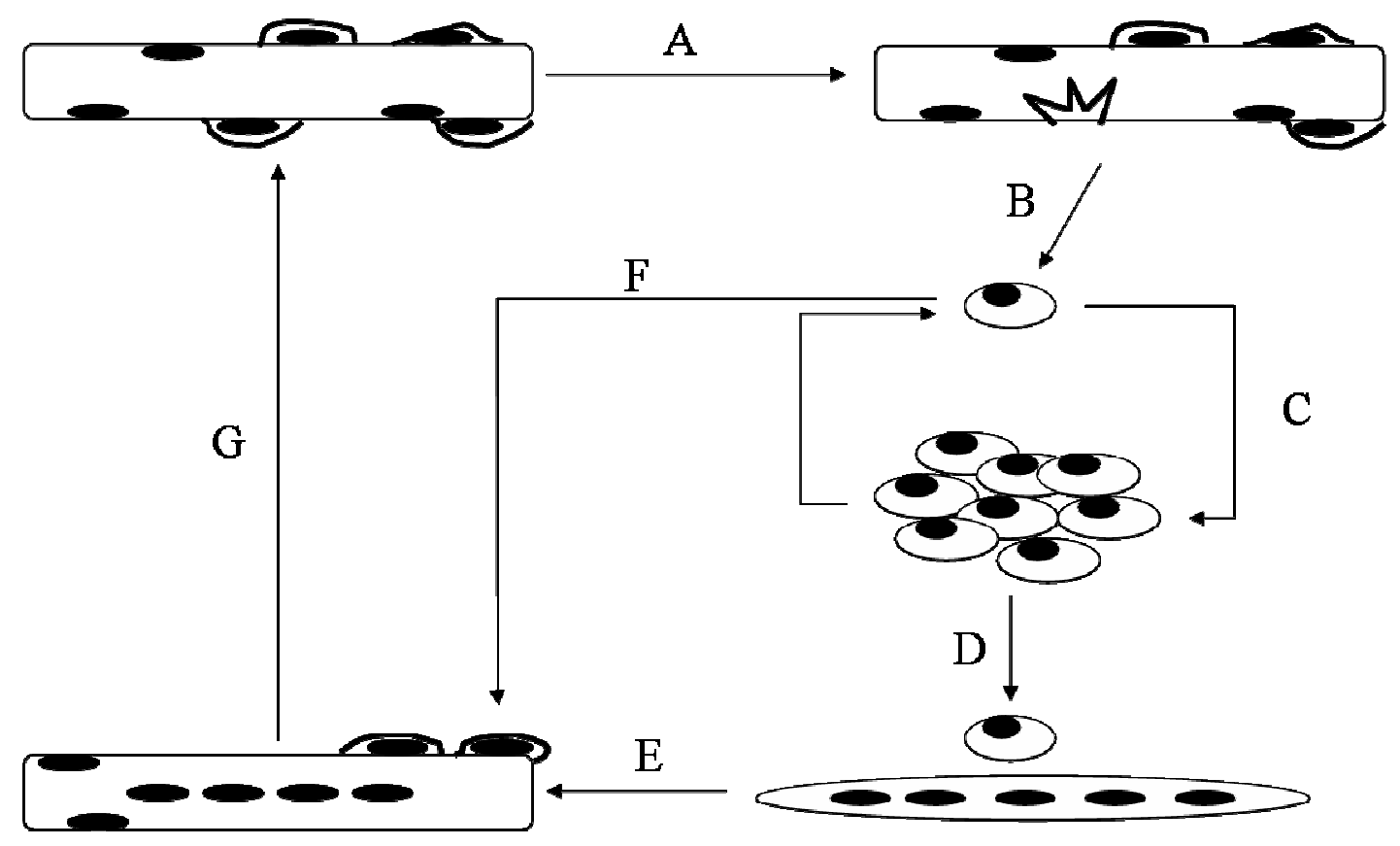

Figure 1.15 Schematic representations of events regulating satellite cell activation. Following muscle damage (A) quiescent satellite cells are activated to enter the cell cycle and proliferate and (B) is followed by terminal differentiation. (C-E) Cells then combine with damaged myofibres for repair or each other for new myofibre formation. (F) A subset of cells return to quiescent state to replenish the satellite cell pool. (G) Repaired or new fibres resemble original myofibres (adapted from Charge and Rudnicki 2004). 
Primary regulators of satellite cell activation include the myogenic regulatory factor (MRF) family of transcription factors and cell cycle kinases, which provide molecular landmarks for the transition from satellite cell quiescence to activation, proliferation and differentiation (Zammit et al. 2004). The best characterised of the MRF's are the myogenic differentiation (MyoD) and myogenin (MyoG) transcription factors. Activation of satellite cells in skeletal muscle is associated with rapid up-regulation of MyoD prior to cell proliferation and continues through differentiation, while MyoG up-regulation occurs in cells initiating the terminal differentiation programme (Cornelison and Wold 1997; Cornelison et al. 2000).

Expression patterns from functionally overloaded skeletal muscle reveal that satellite cells express both MyoD and MyoG during the hypertrophy process and activated satellite cells can significantly contribute to activity-dependent muscle growth (Ishido et al. 2004b; Li et al. 2006; Petrella et al. 2006). A single bout of contractile activity is sufficient to increase cell cycle kinases and MyoD and MyoG mRNA abundance in skeletal muscle of both rodents and humans (Adams et al. 1999; Haddad and Adams 2002; Willoughby and Nelson 2002; Psilander et al. 2003; Bickel et al. 2005; Vissing et al. 2005; Yang et al. 2005). Moreover, this response does not appear to be attenuated with chronic resistance training ( $3 \mathrm{~d} / \mathrm{wk}$ for $16 \mathrm{wk}$ ) which induced MyoD and MyoG mRNA responses equal to a single resistance training bout (Kosek et al. 2006). This implicates the MRF's in contributing to the myogenic programme and resultant compensatory hypertrophy response with resistance training. However, increased MyoD and MyoG expression has also been observed following low-frequency electrical stimulation and endurance exercise (Kadi et al. 2004; Siu et al. 2004; Vissing et al. 2005; Yang et al. 2005). Endurance cycling / swimming is unlikely to induce muscle damage and subsequent satellite cell activation; hence the MyoD / MyoG response to an endurance exercise stimulus has been postulated to regulate a pathway for exercise-induced changes in mitochondrial enzymes and / or muscle fibre type transformation (Kadi et al. 2004; Siu et al. 
2004; Vissing et al. 2005). While there is an established role for MRF's in satellite cell activation and subsequent increases in myonuclei number and myofibre size, it appears these transcription factors have additional and as yet undefined roles in skeletal muscle adaptation to exercise.

\section{Atrophy}

Skeletal muscle atrophy is characterised by a decrease in structural and contractile protein content and fibre diameter (Jackman and Kandarian 2004; Bassel-Duby and Olson 2006). Moreover, while hypertrophy pathways may suppress the activity of some mediators of protein breakdown, atrophy is not simply the reversal of hypertrophy but comprises unique mechanisms in a series of pathways regulating proteolysis (Sacheck et al. 2004; Glass 2005). Atrophy occurs when protein degradation exceeds protein resynthesis and is often prevalent in conditions such as inactivity, aging and disease (Jackman and Kandarian 2004; Reid 2005). The variety of conditions that generate a common outcome suggests that both reciprocal and distinct molecular signalling pathways contribute to the skeletal muscle atrophy programme (Jackman and Kandarian 2004).

Current understanding of skeletal muscle atrophy implicates at least three systems in the regulation of proteolysis. The calcium-dependent protease calpain family and proteolytic caspase class of proteins have been proposed to mediate skeletal muscle myofibrillar disassembly and cleavage of actomyosin protein, respectively (Tischler et al. 1990; Du et al. 2004). Similarly, cathepsin, a proteolytic enzyme involved in lysosomal proteolysis has been implicated in the degradation of membrane proteins such as receptors, channels and transporters (Jackman and Kandarian 2004). While the initial fragmentation of structural and contractile protein via calpain, caspase or lysosomal pathways is required to enable degradation, the destruction of the protein fragments appears to be co-ordinated by a common system (Bodine et al. 2001a; Lecker et al. 2004). 
The destruction of myofibrillar protein is primarily carried out by the ATP-dependent ubiquitin proteasome pathway (Kandarian and Jackman 2006). This pathway labels proteins by conjugation with ubiquitin and this composite protein is subsequently recognised and degraded via zinc-finger domain protein ZNF216 association with the $26 \mathrm{~S}$ proteasome enzyme complex (Reid 2005; Hishiya et al. 2006). In addition, ubiquitin ligases may also degrade MyoD and inhibit subsequent satellite cell activation (Abu Hatoum et al. 1998; Mitchell and Pavlath 2004; Tintignac et al. 2005). Work by Bodine and colleagues (2001) and Gomes and co-workers (2001) has identified important ubiquitin ligase proteins that are upregulated during skeletal muscle atrophy; the muscle-specific atrophy $\mathrm{F}$ box (MAFBx, also known as atrogin-1) and muscle ring finger (MuRF) proteins. The evidence supporting their proposed role in muscle atrophy is compelling as the increase in gene expression of these ubiquitin proteins has been systematically induced with fasting, cachexia, diabetes, uremia, denervation, glucocorticoid release and disuse atrophy models (Bodine et al. 2001a; Jones et al. 2004; Lecker et al. 2004; Sacheck et al. 2004). The results from these studies suggest that the MAFBx and MuRF proteins are valid and reliable markers of skeletal muscle atrophy. However, the molecular proteins that initiate MAFBx and MuRF gene expression are still largely unknown (Figure 1.16).

Principle candidates implicated in MAFBx and MuRF activation include the forkhead (FoxO) transcription factors and TNF $\alpha$ via p38 MAPK and NFkB (Glass 2005). FoxO has numerous regulatory sites mediated by AKT phosphorylation which stimulates the nuclear exclusion and inactivation of this transcription factor (Rena et al. 2002). Constitutively active FoxO acts on the MAFBx promoter to initiate MAFBx transcription and results in dramatic atrophy of myotubes and myofibres (Sandri et al. 2004). Moreover, in the absence of IGF-1 signalling MAFBx and MuRF gene expression and muscle atrophy is increased, implicating AKT-FoxO interactions in mediating regulation of ubiquitin ligases (Sacheck et al. 2004; Stitt et al. 2004). Similarly, activation of NFאB through transgenic expression of IKK $\beta$, and 


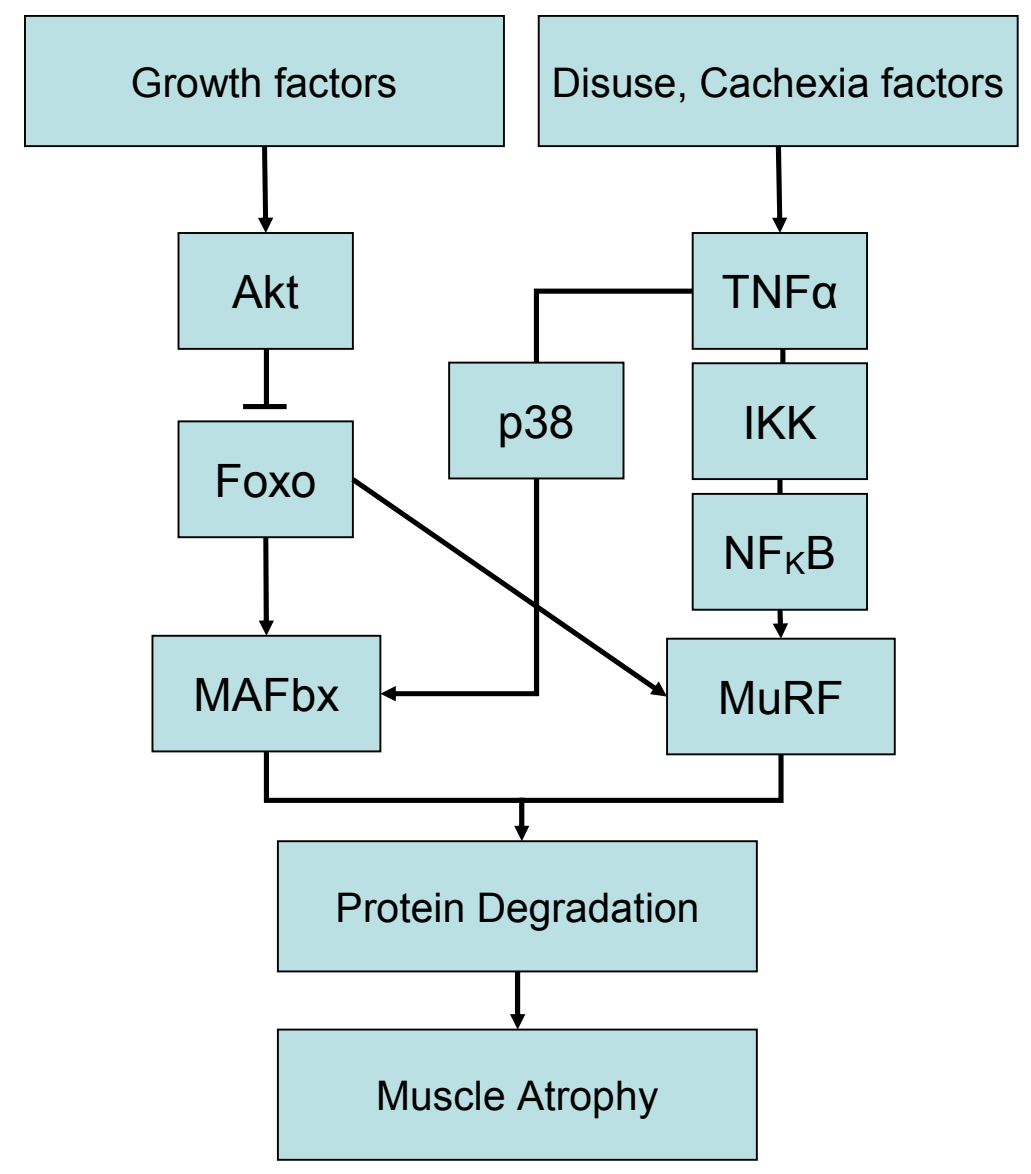

Figure 1.16 Putative pathways regulating signalling to MAFBx / MuRF ubiquitin ligases. MAFBx, muscle atrophy $\mathrm{F}$ box protein; MuRF, muscle ring finger protein; IKK, inhibitor kappa B kinase; $\mathrm{NF \kappa B}$, nuclear factor kappa $\mathrm{B}$; TNFa, tumor necrosis factor alpha; FoxO, forkhead box O; p38, p38 mitogen activated protein kinase (adapted from Cai et al. 2004).

reactive oxygen species stimulated p38 MAPK activity, have been shown to increase MuRF and MAFBx gene expression, respectively (Cai et al. 2004; Li et al. 2005). Pharmalogical inhibition of IKK $\beta$ / NFkB and p38 results in inhibition of MuRF and MAFBx up-regulation and reverses muscle atrophy, providing further evidence for $\mathrm{NF} \kappa \mathrm{B}$ and $\mathrm{p} 38$ regulation of MuRF and MAFBx activation in the atrophy programme, respectively (Cai et al. 2004; Li et al. 2005). 
Myostatin (or growth and differentiation factor 8) is a transforming growth factor- $\beta$ family member that may not be classified as an atrophy-inducing factor per se but functions as an inhibitor of muscle hypertrophy (McNally 2004; Wagner et al. 2005). The role of myostatin as a negative regulator of hypertrophy is highlighted by the extraordinary increase in muscle mass of myostatin-deficient animals and humans (McPherron and Lee 1997; Lee and McPherron 2001; Schuelke et al. 2004). Furthermore, transgenic over expression of myostatin has been shown to reduce muscle mass, fibre size and myonuclei number, suggesting that increased myostatin expression may have the capacity to exacerbate atrophy (Reisz-Porszasz et al. 2003). Myostatin appears to exert its regulatory effect on muscle mass via inhibition of satellite cell proliferation, differentiation and self-renewal (Thomas et al. 2000; Langley et al. 2002; McCroskery et al. 2003). Therefore, down-regulation of myostatin gene expression can result in hypertrophy and hyperplasia following skeletal muscle regeneration (Lee and McPherron 2001).

The capacity of muscle contraction to repress, reverse or exacerbate various atrophy signalling pathways and catabolic states is still unclear. Early work by Sandri et al. (1997) showed that ubiquitin protein and associated apoptosis in mice increased following one night of voluntary wheel running. Their findings were later corroborated by other studies incorporating repeated bouts of resistance training that also induced a post-exercise increase in skeletal muscle ubiquitin protein content (Stupka et al. 2001; Willoughby et al. 2003). Indeed, Yang and colleagues (2006) observed an increase in MAFBx and MuRF mRNA abundance following a single bout of resistance exercise. Conversely, resistance training has also been shown to reduce MuRF and MAFBx gene expression and restore muscle mass and strength following 2-6 wk disuse atrophy, decreasing ubiquitin ligase mRNA abundance by a minimum $\sim 40 \%$ (Jones et al. 2004; Dupont-Versteegden et al. 2006). Myostatin gene expression also appears to be reduced following resistance exercise in humans (Roth et al. 2003; Kim et al. 2005; Raue et al. 2006), although a single study has reported an increase in 
myostatin mRNA abundance after 12 wk resistance training (Willoughby 2004). Moreover, Matsakas and co-workers (2005) observed a decrease in myostatin mRNA in rodents following one and three days $(2 \times 60 \mathrm{~min} / \mathrm{d})$ of swimming training. The authors attributed this somewhat unexpected finding to the need for skeletal muscle remodelling with repeated concentric contractile activity (Matsakas et al. 2005).

Interestingly, Haddad and colleagues (2006) provide evidence that an isometric training programme previously shown to induce hypertrophy (Adams et al. 2004) failed to maintain myofibril protein content during hind limb suspension, despite completely abating the increase in MAFBx, MuRF and myostatin mRNA. These workers suggest that to prevent muscle atrophy, maintaining optimal activity of hypertrophy pathways is required in addition to inhibiting the catabolic stimulus. Furthermore, training history is likely to alter the response of atrophic gene expression where acute bouts of resistance exercise in untrained muscle may induce an atrophy programme due to muscle damage, but may also be required for regeneration remodelling. Indeed, chronic resistance training may ultimately produce a decrease in atrophy gene expression thereby enhancing the capacity for compensatory hypertrophy.

Collectively, these findings suggest that exercise-induced skeletal muscle contraction is capable of altering regulators of skeletal muscle atrophy including pathways regulating ubiquitin ligase and satellite cell activation. However, the interaction of mode of contraction, duration and intensity of exercise likely modifies the specific response of atrophy and / or hypertrophy pathways. In addition, exercise interventions in models of disease or disuse may induce molecular responses that do not accurately reflect changes in atrophy / hypertrophy pathways that occur with resistance training in healthy or athletic populations. Regardless, the proteolytic status in skeletal muscle is the result of the interactions between integrated atrophy and hypertrophy pathways generating a specific time-course of events determining net protein balance that may promote or inhibit protein synthesis (Figure 1.17). 


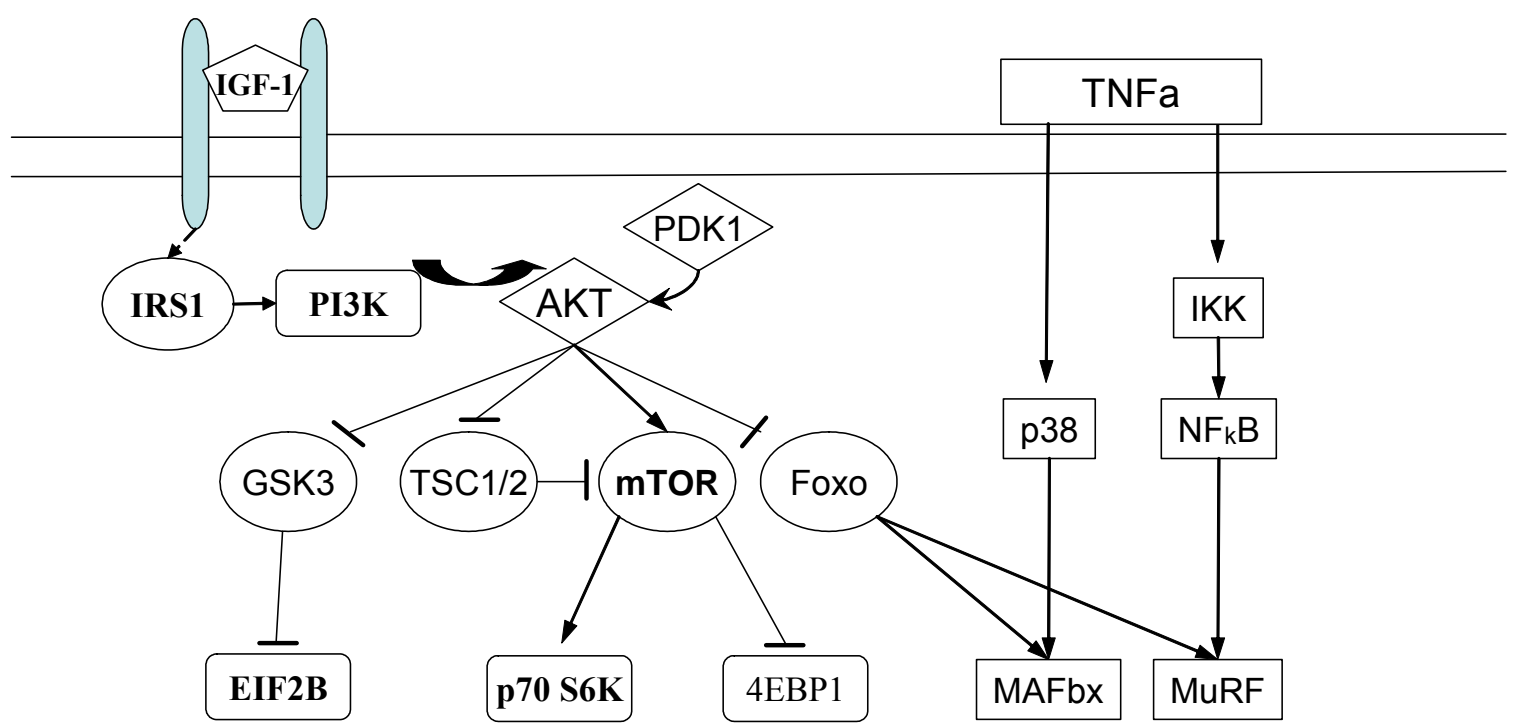

Figure 1.17 Schematic providing an example of the integration of hypertrophy (IGF-1) and atrophy $(\mathrm{TNF} \alpha)$ pathways in skeletal muscle. Bars denote inhibition and arrows denote activation. IRS1, insulin receptor substrate 1; PI3K, phosphatidylinositol-3-OH kinase; PDK1, 3' phosphoinositide-dependent protein kinase 1; FoxO, forkhead box O; mTOR; mammalian target of rapamycin; GSK3, glycogen synthase kinase 3; TSC1/2, tuberous sclerosis complex 1/2; 4EBP1, eukaryotic initiation factor 4E-binding protein; eIF2B eukaryotic initiation factor 2B; p70 S6K, p70 ribosomal S6 kinase; MAFBx, muscle atrophy F box protein; MuRF, muscle ring finger protein; IKK, inhibitor kappa B kinase; NFאB, nuclear factor kappa $\mathrm{B}$; TNFa, tumor necrosis factor alpha; p38, p38 mitogen activated protein kinase (adapted from Glass 2005).

\subsubsection{Concurrent Training}

The concomitant integration of endurance and resistance training in a periodised training plan is termed concurrent training. To date, few animal studies have attempted to elucidate mechanisms of adaptation in skeletal muscle with concomitant aerobic and hypertrophic stimuli. Furthermore, the results of studies investigating adaptation and performance changes in humans undertaking concurrent strength and endurance training have been equivocal (Millet et al. 2002; Balabinis et al. 2003; Häkkinen et al. 2003; Glowacki et al. 2004). There are several possible reasons for this 
discrepancy, including differences in the design of the concurrent training interventions, the training status of the subjects, the influence of dependent variable selection, small sample sizes and inadequate statistical power (Leveritt et al. 2003). Most concurrent training studies have involved cycling, with only a few studies using running or swimming protocols, and have typically employed untrained or moderately trained male volunteers, who undergo short-term (8 to $16 \mathrm{wk}$ ) training interventions in which the training volume and intensity is low. Nevertheless, since the classic investigative work of Hickson (1980) numerous studies have reported a variety of adaptive consequences when combining resistance and endurance training (Table 1.4; Leveritt et al. 1999).

The conundrum of variable results arising from concurrent training research is not surprising given the complexity of signalling and gene expression with endurance and resistance training described previously. Indeed, it is reasonable to suggest that the specific adaptations to the divergent exercise modes appear to be incompatible, at least at the cellular / molecular level. Heavy resistance training does not lead to mitochondrial biogenesis, and the larger myofibril cross sectional area increases diffusion distances for oxygen and substrates (Hood 2001). Therefore, with respect to alterations in the muscle milieu, this does not induce a favourable adaptation for endurance capacity. Likewise, chronic endurance training does not have a marked effect on myofibril size and the muscles altered $\left[\mathrm{AMP} / \mathrm{Ca}^{2+}\right]$ and subsequent residual fatigue may have a negative effect on muscle protein synthesis and the ability to generate force. Accordingly, this does not produce an adaptation conducive for increased muscular size and strength. 
Table 1.4 Summary of research investigating the effect of concurrent strength and endurance training on skeletal muscle adaptation.

\begin{tabular}{|c|c|c|c|}
\hline Author & WK & Training & Concurrent Training Interference? \\
\hline Izquierdo et al. (2005) & 16 & (S) $2 \mathrm{~d} /$ wk (E) $2 \mathrm{~d} / \mathrm{wk}(\mathrm{C}) 1 \times \mathrm{S}+1 \times \mathrm{E}$ once weekly & Yes - lower leg strength development \\
\hline Glowacki et al. (2004) & 12 & (S) 2-3 x wk (E) 2-3 x wk (C) $5 \times$ wk & Yes - reduced increase of maximum oxygen consumption in $\mathrm{C}$ vs. $\mathrm{E}$ \\
\hline Häkkinen et al. (2003) & 21 & (S) $2 \mathrm{~d} / \mathrm{wk}(\mathrm{C}) 2 \mathrm{x} \mathrm{S}+2 \times \mathrm{E}$ & Yes - impaired explosive strength development \\
\hline Balabinis et al. (2003) & 7 & (S) $4 \mathrm{~d} / \mathrm{wk}(\mathrm{E}) 4 \mathrm{~d} / \mathrm{wk}(\mathrm{C}) \mathrm{S}+\mathrm{E}$ regimens / same day & No \\
\hline Millet et al. (2002) & 14 & (E) triathlon precondition phase $(\mathrm{C}) \mathrm{E}+\mathrm{S}-2 \mathrm{x}$ wk & No \\
\hline McCarthy et al. (2002) & 10 & (S) $3 \mathrm{~d} / \mathrm{wk}(\mathrm{E}) 3 \mathrm{~d} / \mathrm{wk}(\mathrm{C})$ combined $\mathrm{S}+\mathrm{E}$ in same session & No \\
\hline Bell et al. (2000) & 12 & (S) $3 \mathrm{~d} / \mathrm{wk}(\mathrm{E}) 3 \mathrm{~d} / \mathrm{wk}(\mathrm{C}) \mathrm{S}+\mathrm{E}$ regimens / alternate day & Yes - impaired strength gains in C compared with $\mathrm{S}$ \\
\hline Horne et al. (1997) & 12 & (S) $3 \mathrm{~d} / \mathrm{wk}(\mathrm{E}) 3 \mathrm{~d} / \mathrm{wk}$ (C) $\mathrm{S}+\mathrm{E}$ regimens / alternate day & Yes - impaired strength gains in $\mathrm{C}$ compared with $\mathrm{S}$ \\
\hline Kraemer et al. (1995) & 12 & (S) $4 \mathrm{~d} / \mathrm{wk}(\mathrm{E}) 4 \mathrm{~d} / \mathrm{wk}$ (C) S + E regimens / same day & Yes - impaired strength gains in $\mathrm{C}$ compared with $\mathrm{S}$ \\
\hline McCarthy et al. (1995) & 10 & (S) $3 \mathrm{~d} / \mathrm{wk}(\mathrm{E}) 3 \mathrm{~d} / \mathrm{wk}(\mathrm{C})$ combined $\mathrm{S}+\mathrm{E}$ in same session & No \\
\hline Hennessy \& Watson (1994) & 8 & (S) $3 \mathrm{~d} / \mathrm{wk}(\mathrm{E}) 4 \mathrm{~d} / \mathrm{wk}(\mathrm{C})$ random $\mathrm{S}+\mathrm{E}$ combined \& separate $5 \mathrm{~d} / \mathrm{wk}$ & Yes - impaired strength gains in $\mathrm{C}$ compared with $\mathrm{S}$ \\
\hline Nelson et al. (1990) & 20 & (S) $4 \mathrm{~d} / \mathrm{wk}$ (E) $4 \mathrm{~d} / \mathrm{wk}$ (C) S + E regimens / same day & Yes - reduced increase of maximum oxygen consumption in $\mathrm{C}$ vs. $\mathrm{E}$ \\
\hline Hunter et al. (1987) & 12 & (S) $4 \mathrm{~d} / \mathrm{wk}(\mathrm{E}) 4 \mathrm{~d} / \mathrm{wk}(\mathrm{C}) \mathrm{S}+\mathrm{E}$ regimens / same day & Yes - reduced vertical jump in C compared with $S$ \\
\hline Dudley \& Djamil (1985) & 7 & (S) $3 \times$ wk (E) $3 \times w k(C) S+E$ regimens / alternate day & Yes - reduced maximum torque at high velocities in $\mathrm{C}$ vs. $\mathrm{S}$ \\
\hline Hickson (1980) & 10 & (S) $5 \mathrm{~d} / \mathrm{wk}(\mathrm{E}) 6 \mathrm{~d} / \mathrm{wk}(\mathrm{C}) \mathrm{S}+\mathrm{E}$ regimens / same day & Yes - impaired strength gains in $\mathrm{C}$ compared with $\mathrm{S}$ group \\
\hline
\end{tabular}

S, strength training; E endurance training; C, concurrent strength and endurance training 
The majority of research aimed at elucidating the adaptive responses to concurrent training has been confined to "end state" measures such as maximum strength / power or maximal aerobic capacity. Consequently, it is not possible to deduce the timing and identity of the regulatory events that orchestrated the observed "end-point" adaptations. As a result there has been little or no elucidation of the mechanisms underlying the specificity of training adaptation or interference to these pathways during concurrent training. Early work by Riedy and colleagues (1985) examined whether enlarged rodent skeletal muscle following $30 \mathrm{~d}$ surgical ablation responded to endurance training similar to control muscle. They observed comparable increases in succinate dehydrogenase activity in enlarged versus normal muscle and concluded that the response to endurance training was unaltered in hypertrophied muscle. Conversely, Stone and co-workers (1996) assessed the effect of 6 wk concurrent surgical overload and endurance training on citrate synthase and observed compromised oxidative enzyme content with concomitant hypertrophy. Finally, Putman and colleagues (2004; Putman et al. 2004) employed 12 wk concurrent training to determine changes in myosin heavy chain isoform content. These workers reported reduced strength development with concurrent compared to strength training alone and greater fast-to-slow fibre-type transition and attenuated hypertrophy of type I fibres following concurrent training. Accordingly, these inconclusive findings provide little mechanistic insight regarding the possible interference and / or incompatibility of adaptation with concurrent strength and endurance training. However, emerging evidence provides a number of possible mechanisms that may, in part, offer a rationale for adaptation specificity and an "interference" phenomenon with concurrent training.

The elongation phase of translation is mediated by eukaryotic elongation factors which represent a rate limiting step in protein synthesis (Bouchard et al. 1997). A key component of this translational machinery is eukaryotic elongation factor 2 (eEF2) which mediates translocation of the ribosome along the mRNA. eEF2 is phosphorylated and inactivated by 
eEF2K in response to stimuli that increase energy demand or reduce energy supply (Browne and Proud 2002). Moreover, the activation of eEF2K appears to be regulated upstream via calmodulin and AMPK mediated signalling, kinases activated in response to endurance exercise (Nairn and Palfrey 1987; Ryazanov 1987; Horman et al. 2002). Indeed, Rose and colleagues (2005) provide strong evidence for the phosphorylation and inactivation of eEF2 in a calcium-calmodulin dependent manner in response to 90 min submaximal cycling $(\sim 67 \%$ $\mathrm{VO}_{2 \text { peak }}$ ). Atherton and co-workers (2005) likewise observed an increase in eEF2 phosphorylation immediately after, and $3 \mathrm{~h}$ following an endurance-like stimulus. This suggests that inhibition of eEF2 activity by endurance exercise results in a decrease in translation elongation and protein synthesis (Figure 1.18). Conversely, IGF-1 signalling has been implicated in the phosphorylation and inhibition of eEF2K and subsequent activation of eEF2 (Browne and Proud 2002). Data exists showing phosphorylation at serine ${ }^{366}$ by p70 S6K inactivates eEF2K, while mTOR has also been shown to phosphorylate serine ${ }^{78}$ on eEF2K decreasing kinase activity (Wang et al. 2001; Browne and Proud 2004). Therefore, in addition to its established role in translation initiation these findings also implicate mTOR-p70 S6K in the regulation of eEF2 and translation elongation. Increased mTOR and p70 S6K activity following resistance training would be expected to promote hypertrophy in part via increased eEF2 activity (Figure 1.18). In support of this contention a resistance-like stimulus has been demonstrated to decrease eEF2 phosphorylation likely enhancing elongation and protein synthesis (Atherton et al. 2005), but this effect has yet to be investigated in humans. Nonetheless, contrasting regulation of eEF2 mediated elongation by endurance and resistance training may represent a point of divergence for control of protein synthesis. 


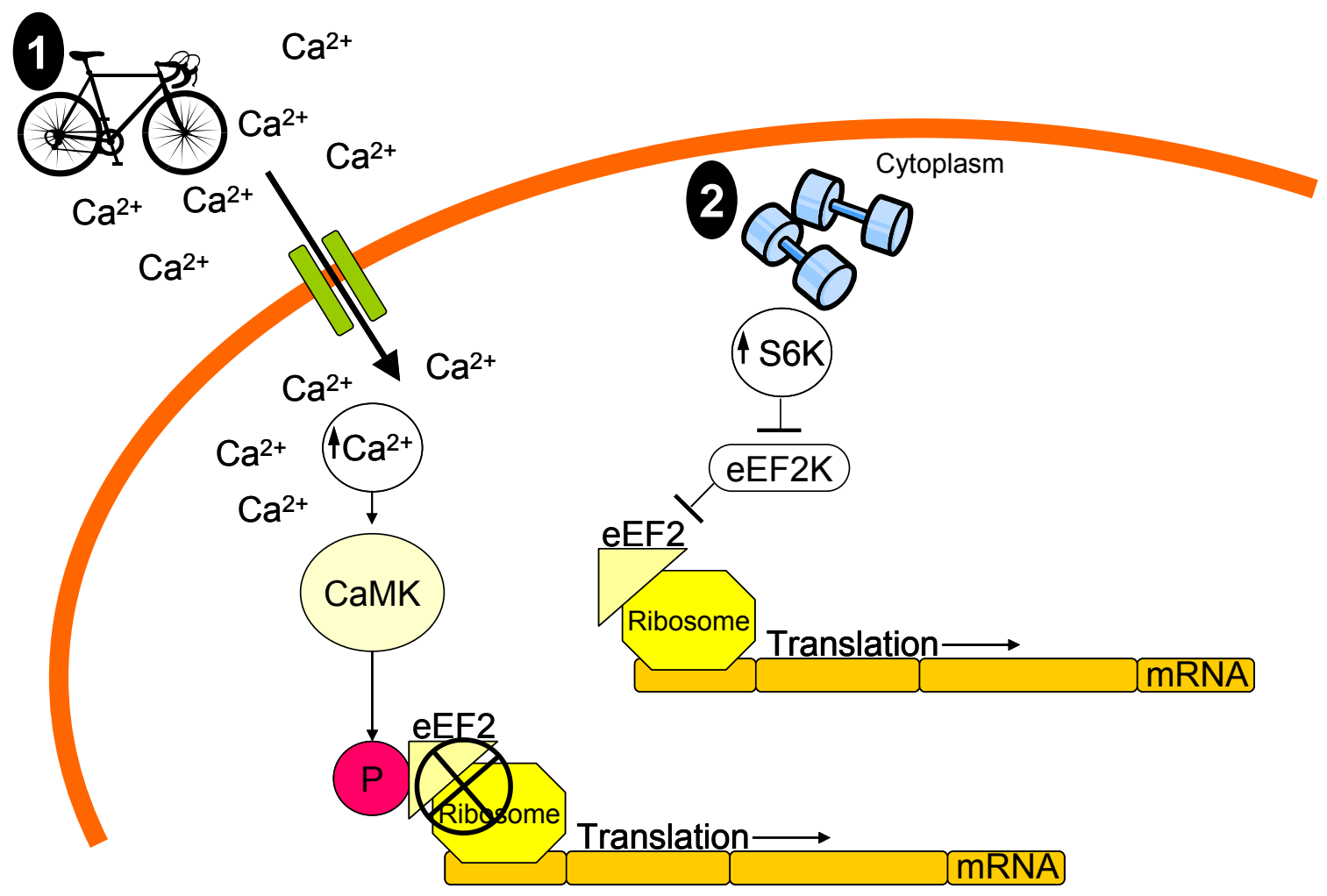

Figure 1.18 Putative divergent regulation of eukaryotic elongation factor 2 (eEF2) by (1) endurance and (2) resistance exercise. Bars denote inhibition and arrows denote activation. CaMK, calmodulin-dependent kinase; S6K, ribosomal protein S6 kinase; eEF2K, eukaryotic elongation factor 2 kinase (based on findings of Atherton et al. 2005; Rose et al. 2005; and Wang et al. 2001).

The FoxO transcription factor has been implicated in promoting mRNA abundance of genes involved in processes as varied as mitochondrial biogenesis and myofibrillar protein degradation (Glass 2005; Hood et al. 2006). FoxO functions on the promoter regions and initiates transcription of a number of genes including PGC-1 $\alpha$ and MAFBx, and the activity and nuclear abundance of FoxO is regulated by AKT (Daitoku et al. 2003; Sandri et al. 2004; Nader 2005). When AKT phosphorylates FoxO it translocates from the nucleus to the cytosol and is prevented from promoting transcription and may also be subject to cytosolic proteasomal degradation (Matsuzaki et al. 2003). AKT activation following resistance exercise would likely result in phosphorylation of FoxO and subsequent inhibition of 
ubiquitin ligase gene expression (Figure 1.19). While these events would be expected to promote hypertrophy, work by Southgate and co-workers (2005) suggests that this results in the concomitant down-regulation of PGC-1 $\alpha$ gene expression (Figure 1.19). Equally, endurance exercise is associated with increased PGC- $1 \alpha$ gene expression and mitochondrial biogenesis promoting an oxidative phenotype (Irrcher et al. 2003). However, the nuclear location of FoxO with endurance exercise may suppress net protein synthesis due to increased activity of ubiquitin gene expression and subsequent protein degradation. Therefore, altered regulation of FoxO activity with contrasting modes of exercise may generate contradictory gene expression profiles ultimately reducing the specificity of adaptation.

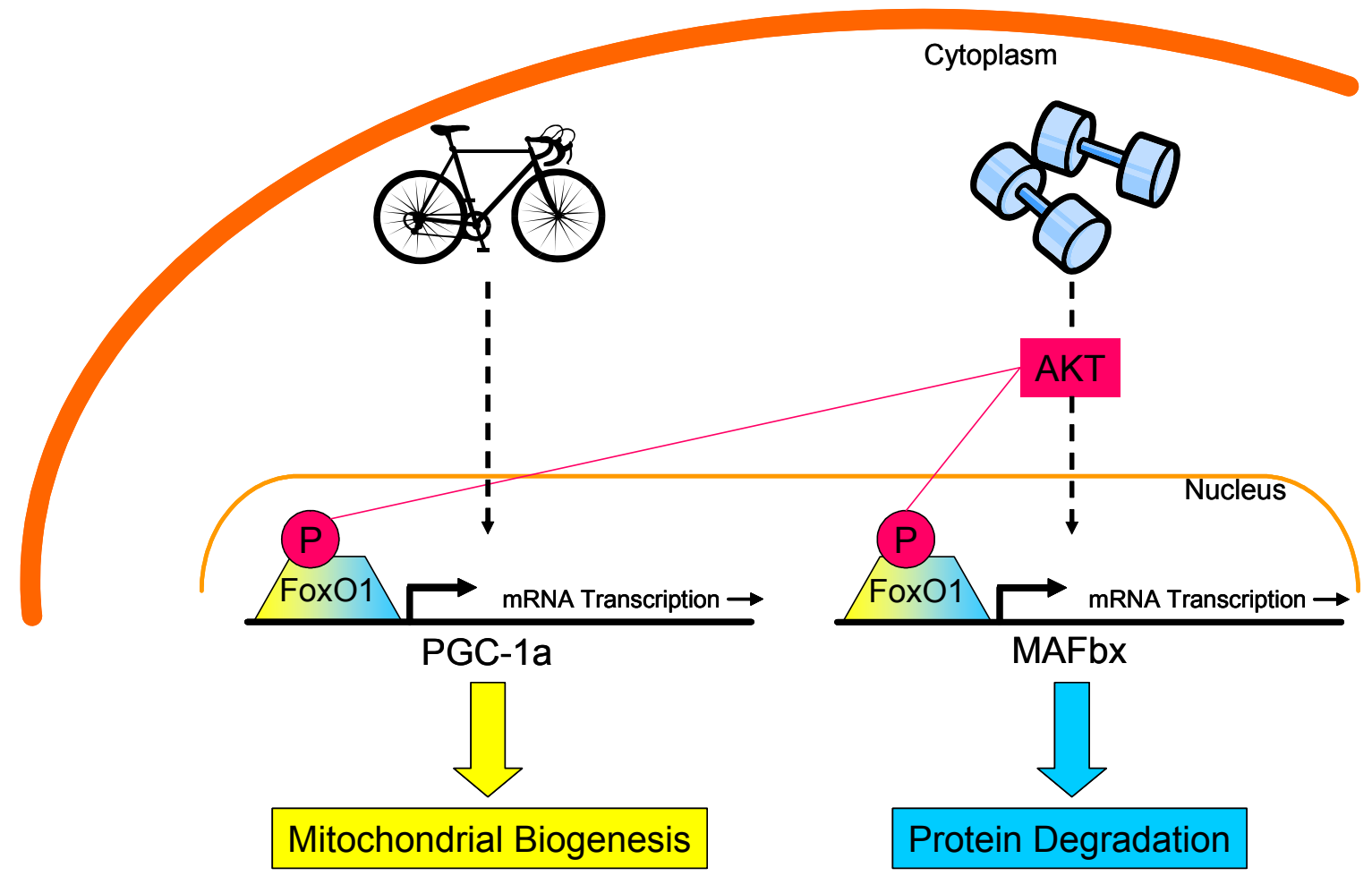

Figure 1.19 Proposed effects of acute endurance and resistance training on forkhead box O1 (FoxO1) mediated transcription. PGC-1 $\alpha$, peroxisome proliferator activated receptor gamma co-activator-1 alpha; MAFBx, muscle atrophy $\mathrm{F}$ box protein (based on findings of Hoffman \& Nader 2004 and Southgate et al. 2005). 


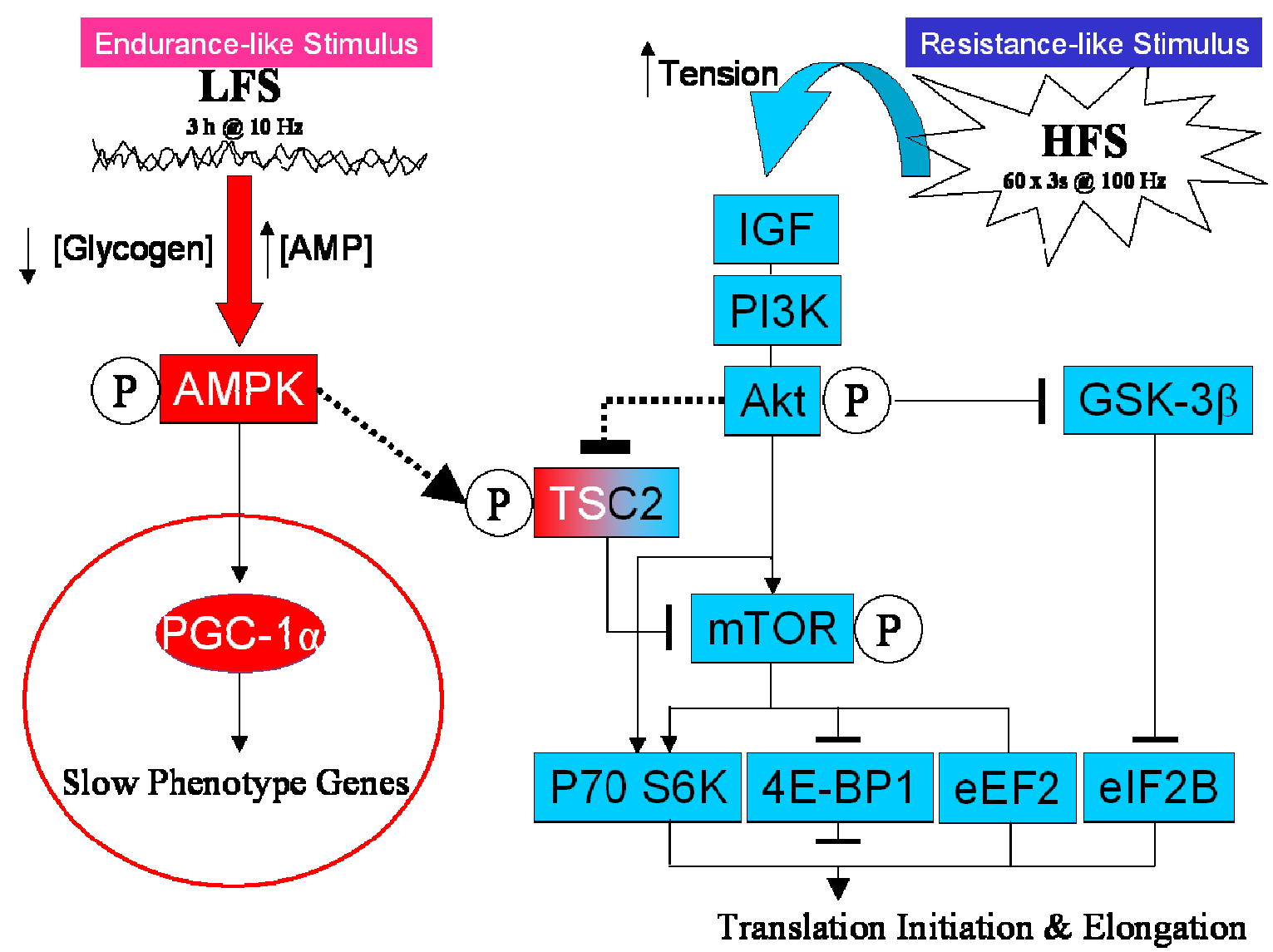

Figure 1.20 Putative adaptive pathways in response to endurance- and resistance-like stimuli. Bars denote inhibition and arrows denote activation. AMPK, adenosine monophosphate kinase; PGC-1 $\alpha$, peroxisome proliferator activated receptor gamma co-activator-1 alpha; IGF, insulin-like growth factor; PI3K, phosphatidylinositol-3-OH kinase; mTOR, mammalian target of rapamycin; GSK3, glycogen synthase kinase 3; TSC1/2, tuberous sclerosis complex 1/2; 4E-BP1, eukaryotic initiation factor 4E-binding protein; eIF2B eukaryotic initiation factor 2B; p70S6K, p70 ribosomal S6 kinase; eEF2, eukaryotic elongation factor (adapted from Atherton et al. 2005).

The most compelling mechanism proposed to mediate the specificity of training and subsequent interference effect with concurrent training may be the AMPK-AKT "masterswitch" hypothesis of Atherton and colleagues (Figure 1.20). In a rodent model in which muscle fibres were electrically stimulated for prolonged periods at low frequency (to mimic endurance training) or for short periods with high-frequency (to mimic resistance training) 
they observed a reciprocal relationship in the activation of AMPK and AKT pathways in response to these extreme divergent stimuli. Specifically, after low-frequency stimulation they observed increased AMPK-TSC2 activity, PGC-1 $\alpha$ gene expression and an inhibition of mTOR mediated translation initiation. Conversely, after high-frequency stimulation there was increased AKT-mediated hypertrophy signalling concomitant with a decrease in AMPK and suppression of TSC2 activity. Based on these findings in a single muscle electrical stimulation model to mimic exercise, these workers proposed that the AMPK and AKT signalling may represent divergent pathways that when activated direct skeletal muscle adaptation to either an aerobic or hypertrophic phenotype (Figure 1.20).

Collectively, the current literature provides a number of possible mechanisms to explain the specificity of training adaptation in response to strength and endurance exercise. However, as it appears that divergent adaptive phenotypes are induced via the complex manipulation of numerous common signalling and gene expression pathways this highlights the intricacy of adaptation to exercise stimuli. Regardless, alternating exercise modes during concurrent training may reduce the capacity for the simultaneous acquisition of hypertrophy and / or mitochondrial training-induced adaptation responses compared with single mode training. 


\subsection{Summary $\&$ aims of this thesis}

The specificity of training adaptation is highlighted by comparing the distinct adaptive responses in skeletal muscle to endurance versus heavy resistance training. Heavy resistance training increases muscle size and strength while regular endurance training results in enhanced oxygen kinetics and improved resistance to fatigue. It would appear that skeletal muscle adaptive responses are reduced when incorporating concurrent endurance and resistance exercise stimuli, producing moderate alterations in muscle phenotype when compared to single mode training. However, the molecular events that promote or inhibit the specificity of training adaptation are incompletely resolved and determining the interaction of adaptive pathways when undertaking concurrent strength and endurance training remains elusive. Moreover, the current understanding of intracellular signalling and gene expression with various modes of training is limited due to the specific transient and time-dependent molecular responses to exercise. Accordingly, there is a need to better define the acute and chronic responses to exercise by manipulating the mode, volume, intensity and frequency of training thereby elucidating the specific molecular and genetic mechanisms that ultimately determine the specificity of training adaptation.

The aims of this thesis were to 1) systematically evaluate the time-course of a spectrum of cellular and molecular markers specific to the regulation of muscle contractile protein and subsequent hypertrophy, after the imposition of different resistance training protocols and 2) to quantify the early response to divergent training stimuli in competitive athletes who are highly specialised / adapted to a single discipline, and to establish the degree of "signalling specificity" in these athletes. 


\section{Chapter Two}

High-frequency training stimulus attenuates AKT and exacerbates TNF $\alpha$ signalling responses in skeletal muscle

Adapted from: Coffey, V.G., Reeder, D.W., Lancaster, G.I., Yeo, W.K., Febbraio, M.A.,

Yaspelkis III, B.B., Hawley, J.A. American Journal of Physiology Endocrinology and Metabolism (In Review). 


\subsection{Introduction}

Skeletal muscle is the most abundant tissue in the human body and its normal physiology plays a fundamental role in health and disease. During many disease states, a dramatic loss of skeletal muscle mass (atrophy) is observed. In contrast, chronic resistance training results in increased skeletal muscle size (hypertrophy) and changes in muscle contractile properties that ultimately lead to gains in strength and power (Fry et al. 2003). While such adaptations involve multiple factors including nutrient availability, genetic predisposition and environmental factors etc, they are likely to be the result of the cumulative effect of repeated training bouts, with the cellular responses leading to these long-term adaptations occurring during and after each training session (Widegren et al. 1998; Atherton et al. 2005; Yang et al. 2005). Indeed, many contraction-induced signalling events in muscle appear to be maximally activated in the initial (0-4 h) period post-exercise, returning to basal levels within $24 \mathrm{~h}$ (Widegren et al. 1998; Nader and Esser 2001). This short-term, transient increase in signalling activation following contraction represents a stimulus capable of initiating downstream events co-ordinating an increase in gene expression and subsequent protein synthesis (Atherton et al. 2005; Cuthbertson et al. 2005).

The signalling pathways that initiate adaptive processes in skeletal muscle constitute a complex network of kinases and phosphatases that are often subject to a high degree of feedback regulation, cross-talk, and transient activation (Glass 2005). While our current understanding of the signal transduction pathways controlling skeletal muscle hypertrophy and atrophy are incompletely resolved, a growing body of evidence identifies several key signalling cascades that are pivotal to these adaptation processes (Glass 2005). For example, activation of insulin-like growth factor-1 (IGF-1) and AKT / mammalian target of rapamycin / p70 S6 kinase is sufficient to induce skeletal muscle hypertrophy (Adams and McCue 1998; Bodine et al. 2001b), while this signalling cascade can also block the transcriptional upregulation of key mediators of skeletal muscle atrophy (Latres et al. 2005), the ubiquitin- 
ligases MuRF1 and MAFBx (also called Atrogin-1). Activation of the NFkB transcription pathway, by cachectic factors such as TNF $\alpha$ and inhibitor NFkB kinase, is sufficient to induce skeletal muscle atrophy, in part via NFkB-mediated up-regulation of MuRF1 (Cai et al. 2004).

Much of the work that has attempted to elucidate the signalling pathways regulating hypertrophy / atrophy in skeletal muscle has utilised transgenic manipulation, chronic unloading or electrical stimulation. Such models may not be typical of the loading patterns encountered with daily physical activity. To date, few studies have incorporated in vivo exercise-induced contractile activity as a stimulus to examine changes in growth factor and inflammatory signalling cascades. The present study compared a conventional-frequency resistance training regimen with a novel high-frequency "stacking" protocol designed to generate a summation of transient exercise-induced signalling responses in order to identify the discrete cellular and molecular responses that take place following repetitive overload. The hypothesis was that when high-frequency resistance training sessions were performed with short (i.e. $3 \mathrm{~h}$ ) recovery periods, the transient response of key signalling pathways initiating hypertrophy may be extended and additive compared to when the same work was undertaken with longer (i.e. $48 \mathrm{~h}$ ) recovery.

\subsection{Methods}

\section{Experimental Design}

Seventy-two male Sprague-Dawley rats $\sim 6$ wk of age (mass $351 \pm 17 \mathrm{~g}$ ) were obtained from Harlan (San Diego, CA) and housed four per cage in an environment maintained at $\sim 21{ }^{\circ} \mathrm{C}$ with an artificial 12:12 h light-dark cycle. All animals received a standard chow diet $(63 \%$ carbohydrate, $17 \%$ fat and $20 \%$ protein, no. 112386 , Dyets, Bethlehem, PA) for the duration of the study and had ad libitum access to food and water. Animals were assigned to one of three treatment groups: control (Con, $\mathrm{n}=6)$; high-frequency "stacked" training protocol (HF; $\mathrm{n}=36)$ or conventional-frequency training protocol $(\mathrm{CF} ; \mathrm{n}=30)$. Exercise-trained groups (HF 
and CF) were further subdivided into "stacked" training that completed either one, two, three or four exercise sessions on the same day $(1 \times, 2 \times, 3 \times$ or $4 \times, n=6$ per group) or conventional training, that completed up to three exercise sessions over $5 \mathrm{~d}$ (DAY 1, DAY 3 or DAY $5 \mathrm{n}=$ 6 per group). Following the completion of both HF and CF, two groups of animals were sacrificed $24 \mathrm{~h}$ and $48 \mathrm{~h}$ after their last training bout ( $\mathrm{n}=6$ per group). CF and HF training protocols were matched for total work. HF "stacked" training comprised four bouts of 3 sets $\times$ 10 repetitions separated by $3 \mathrm{~h}$ recovery while CF training consisted of three bouts of 4 sets $\times$ 10 repetitions with $48 \mathrm{~h}$ recovery between resistance training bouts (Figure 2.1). All experimental and training procedures were approved by the Institutional Animal Care and Use Committee at California State University, Northridge and conformed to the guidelines for the use of laboratory animals published by the US Department of Health and Human Resources.
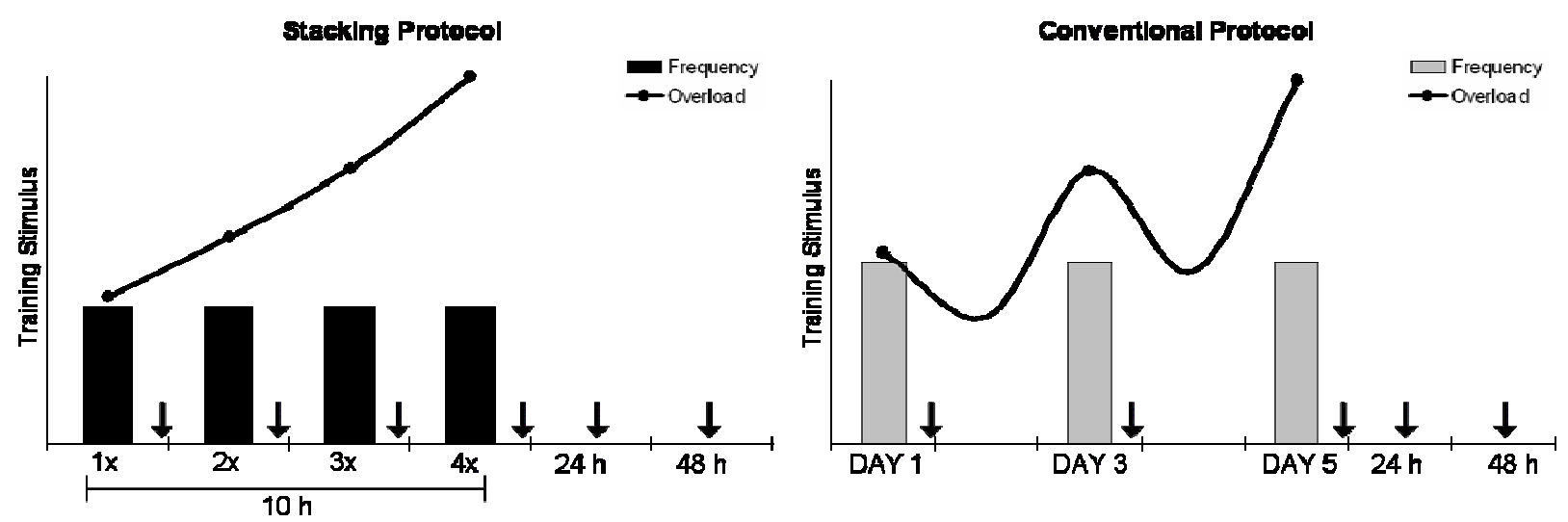

Figure 2.1 Representative schematic of high-frequency "stacking" and conventionalfrequency resistance training protocols. The stacked protocol was comprised of four bouts of $3 \times 10$ repetitions separated by $3 \mathrm{~h}$ recovery and completed within $10 \mathrm{~h}$ on a single day. Conventional training consisted of three bouts of $4 \times 10$ repetitions with $48 \mathrm{~h}$ between resistance training bouts completed over $5 \mathrm{~d}$. Protocols were matched for total work and repetitions were performed at $75 \%$ one-repetition maximum with 3 min recovery between sets. Animal groups $(n=6)$ were sacrificed and white quadriceps muscle extracted $3 \mathrm{~h}$ after every training bout (arrows), and 24 and $48 \mathrm{~h}$ following the final exercise session of each protocol. 
Resistance Training Protocols and Animal Sacrifice

One repetition maximum (1-RM) was determined for all animals a minimum of $5 \mathrm{~d}$ prior to commencement of a training period. The 1-RM established the physical load that could be lifted once but not a second time with the same squat-training equipment that was utilised during all training sessions. Mean 1-RM for all animals was $998 \pm 215 \mathrm{~g}$. All subsequent resistance-training protocols were performed at $75 \%$ of $1-\mathrm{RM}$. The core components of the training protocol and apparatus utilised in the present study have been described in detail previously (Krisan et al. 2004). Briefly, the apparatus consisted of two $45 \mathrm{~cm}$ vertical metal rods $0.5 \mathrm{~cm}$ in diameter set $31 \mathrm{~cm}$ apart on a $15 \mathrm{~cm} \times 20 \mathrm{~cm}$ stainless steel metal base securely inserted into a $2.54 \mathrm{~cm}$ thick wood platform. A $33 \mathrm{~cm} \times 2.54 \mathrm{~cm}$ wood crossbeam was outfitted with two brass sleeves and placed on the two vertical rods, allowing for uninhibited vertical movement. An aluminium holder, moulded to accommodate the rats, was attached to the centre of the crossbeam at a $90^{\circ}$ angle relative to the base. Animals were strapped into a nylon vest and attached to the aluminium holder with Velcro straps before being placed in a squat position using safety stoppers on each rod to support the load. Two $5 \mathrm{~cm}$ metal pegs attached vertically on opposite ends of the crossbeam were used to mount calibrated miniature weight plates. A brief low-voltage electrical stimulus ( $10 \mathrm{~V}, 0.3$-s duration) was delivered by manually depressing a switch that allowed current to flow through an electrode attached to the tail of the animals to initiate each repetition. After each repetition, the animals were repositioned on the apparatus such that their legs were beneath the torso. A repetition was initiated as soon as practicable following each prior repetition (i.e. cessation of movement and re-positioning - mean interval approximately 2 s) and repeated until 10 repetitions were completed. The animals were allowed to rest for $3 \mathrm{~min}$ between each set and were removed from the apparatus and returned to their cage during the recovery period. Group sacrifice ( $\mathrm{n}=$ 6) was conducted $3 \mathrm{~h}$ after every resistance training session for both CF and HF training 
protocols, and 24 and $48 \mathrm{~h}$ following the final exercise session of each protocol, respectively. Animals were sacrificed via cervical dislocation and prepared for hind limb muscle collection. Portions of the white quadriceps (WQ) were surgically removed from the left and right hind limbs in sequence and freeze clamped in liquid $\mathrm{N}_{2}$. Muscle samples were stored at $-80{ }^{\circ} \mathrm{C}$ until subsequent analysis.

Analytical Procedures

Muscle Glycogen Concentration

Muscle samples ( 40-50 mg) were freeze-dried with visible connective tissue removed during powdering. Aliquots $(\sim 3 \mathrm{mg})$ of freeze-dried muscle were extracted with $250 \mu \mathrm{L}$ of $2 \mathrm{M}$ hydrochloric acid, incubated at $100{ }^{\circ} \mathrm{C}$ for $2 \mathrm{~h}$ and then neutralised with $750 \mu \mathrm{L}$ of $0.67 \mathrm{M}$ sodium hydroxide for subsequent determination of glycogen concentration via enzymatic analyses $(50 \mathrm{mM}$ Tris, $25 \mathrm{mM} \mathrm{HCL}, 1 \mathrm{mM} \mathrm{MgCl} 2,0.5 \mathrm{mM}$ DTT, $0.3 \mathrm{mM}$ ATP, $0.05 \mathrm{mM}$ $\mathrm{NADP}$ and $1 \mathrm{U} / \mathrm{ml} \mathrm{HK}, 0.1 \mathrm{U} / \mathrm{ml}$ G-6-PDH) with NADH and glucose standards by fluorometric detection. Glycogen concentration was expressed as millimoles of glycogen $\cdot \mathrm{kg}^{-1}$ d.w.

Western Blotting

Materials

Monoclonal anti-phospho-AKT ${ }^{\text {Ser473 }}$ (\#4058), polyclonal anti-phospho-mTOR ${ }^{\text {Ser2448 }}$ (\#2971), -

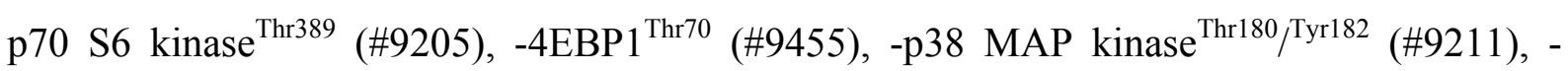
FKHR $^{\text {Ser256 }}(\# 9461)-I K K \alpha / \beta^{\text {ser176/180 }}(\# 2681)$ and polyclonal anti-AKT (\#9272), -p70 S6 kinase (\#9202), -p38 MAP kinase (\#9212), and -TNFa (\#3707) were from Cell Signalling Technology, Inc. (Beverly, MA, U.S.A.). Polyclonal anti-mTOR (\#07-231) was from Upstate Signalling Solutions (Charlottesville, Va, U.S.A.). Anti-phospho-AMPK ${ }^{\text {thr172 }}$ was raised against AMPK alpha peptide (KDGEFLRpTSCGAPNY) as described previously (Clark et al. 
2004). Anti-rabbit secondary antibody and enhanced chemiluminescence reagents were from Amersham Biosciences (Buckinghamshire, U.K.) and Pierce Biotechnology (Rockford, IL, U.S.A.).

\section{Procedure}

Samples were homogenised in ice cold homogenisation buffer containing $50 \mathrm{mM}$ Tris-HCL, $\mathrm{pH}$ 7.5, $1 \mathrm{mM}$ EDTA, $1 \mathrm{mM}$ EGTA, 10\% glycerol, 1\% triton-X, $50 \mathrm{mM} \mathrm{NaF}, 5 \mathrm{mM} \mathrm{Na}$ pyrophosphate, $1 \mathrm{mM}$ DTT, $10 \mu \mathrm{g} / \mathrm{mL}$ trypsin inhibitor, $2 \mu \mathrm{g} / \mathrm{mL}$ aprotinin, $1 \mathrm{mM}$ benzamidine and $1 \mathrm{mM}$ phenylmethylsulfonyl fluoride. The lysate was kept on ice at all times then centrifuged at $12000 \mathrm{~g}$ for $20 \mathrm{~min}$ at $4{ }^{\circ} \mathrm{C}$. The supernatant was transferred to a sterile tube and subsequently aliquoted to determine protein concentration (Pierce, Rockford, IL, U.S.A.). Aliquots were stored at $-80{ }^{\circ} \mathrm{C}$ until analysis. Aliquots of lysate (60-80 $\mu \mathrm{g}$ of protein) were re-suspended in Laemmli sample buffer. Proteins were then separated by SDS/PAGE, transferred to polyvinylidenedifluoride membranes (Bio-Rad, Hercules, CA, U.S.A.), blocked with 5\% non-fat milk for $90 \mathrm{~min}$, washed with TBST $(10 \mathrm{mM}$ Tris $\mathrm{HCl}, 100 \mathrm{mM} \mathrm{NaCl}$, $0.02 \%$ Tween) and incubated with appropriate primary antibodies overnight at $4{ }^{\circ} \mathrm{C}$. Membranes were washed with TBST and incubated with an appropriate secondary antibody for 60 min. Proteins were visualised by chemiluminescence and quantified by densitometry. Samples from each training group were run on the same gel and the amount of protein from the densitometric quantification is expressed as arbitrary units.

\section{Statistical Analysis}

Immunoblot phosphorylation and total protein data are expressed in arbitrary units relative to non-exercise controls. Differences between means were determined by a one-way analysis of variance (ANOVA) with Newman-Keuls post-hoc test (SigmaStat for windows version 3.11). 
All values are expressed as means and standard error (SE) with the critical level of significance established at $\mathrm{P}<0.05$.

\subsection{Results}

Muscle Glycogen

Muscle glycogen content in HF was decreased $\sim 30-35 \% 3 \mathrm{~h}$ after the initial training session and remained below non-exercise control values for each subsequent bout $(\mathrm{P}<0.05$, Figure 2.2A). Muscle glycogen levels were restored to control values after 24 and $48 \mathrm{~h}$ of recovery. $\mathrm{CF}$ resulted in a decline in muscle glycogen $3 \mathrm{~h}$ after training on DAY 1 (NS, Figure 2.2B). After DAY 3 there was an increase in glycogen content compared to control and all time points $(\mathrm{P}<0.05)$, but thereafter muscle glycogen levels were not different to non-exercise control values.

\section{Signalling Responses}

\section{5'Adenosine Monophosphate Activated Protein Kinase (AMPK)}

As might be expected with the decline in muscle glycogen after the initial training bout, phosphorylation of $\mathrm{AMPK}^{\text {thr172 }}$ increased gradually after the HF training bouts $(\sim 25-40 \%)$ reaching significance $3 \mathrm{~h}$ after bout 4 compared to $48 \mathrm{~h}$ post-exercise $(\sim 55 \%, \mathrm{P}<0.05$, Figure 2.2C). In contrast, there were no differences in phosphorylated AMPK ${ }^{\text {thr172 }}$ over time with the CF training protocol (Figure 2.2D).

\section{AKT / Mammalian Target of Rapamycin (mTOR) / Forkhead Box O1 (FoxO1)}

Phosphorylation of $\mathrm{AKT}^{\mathrm{ser} 473}$ was significantly decreased following the second bout of HF training $(\mathrm{P}<0.05)$ and remained suppressed for the remainder of this regimen. This suppression of AKT persisted for up to $48 \mathrm{~h}$ after completion of the HF training protocol (Figure 2.3A). In contrast, CF training had little effect on AKT phosphorylation (Figure 2.3B). mTOR was not significantly altered following either training protocol (Figure $2.3 \mathrm{C}$ and 
2.3D). FoxO $1^{\operatorname{ser} 256}$ phosphorylation was decreased by $\sim 24 \%$ ( $2 \times$ and $\left.3 \times, \mathrm{NS}\right)$ and $\sim 26 \%$ ( $24 \mathrm{~h}$,

NS) with HF and CF resistance training, respectively, but these changes were not significant

(Figure 2.3E and 2.3F).
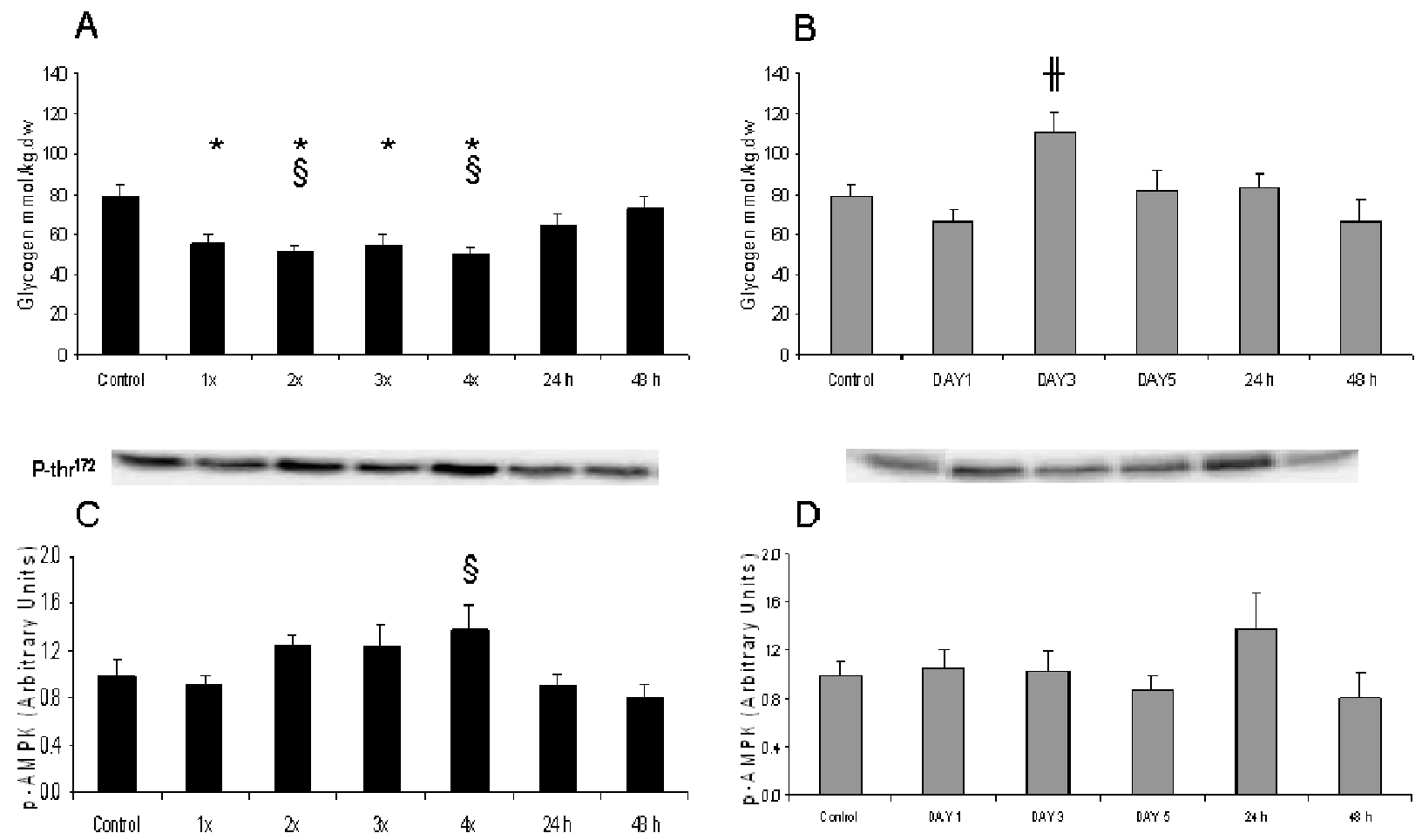

Figure 2.2 Muscle glycogen content (A, B) and 5' adenosine monophosphate activated protein $_{\text {kinase }} \mathrm{e}^{\text {thr172 }}$ (AMPK) phosphorylation (C, D) following either high-frequency "stacked" resistance training comprising four bouts of $3 \times 10$ repetitions separated by $3 \mathrm{~h}$ recovery ( $A, C ; n=6$ per group) or conventional-frequency training consisting of three bouts of $4 \times 10$ repetitions with $48 \mathrm{~h}$ between resistance training bouts (B, $D ; n=6$ per group). Protocols were matched for total work and repetitions were performed at $75 \%$ one-repetition maximum with 3 min recovery between sets. White quadriceps muscle was extracted $3 \mathrm{~h}$ after every training bout, and 24 and $48 \mathrm{~h}$ following the final exercise session of each protocol, respectively. Results are group means $( \pm \mathrm{SE})$ and p-AMPK arbitrary values are expressed relative to control. Significant difference (One-way ANOVA, $\mathrm{P}<0.05$ ) vs. *control; $\S 48 \mathrm{~h}$; \# all. 

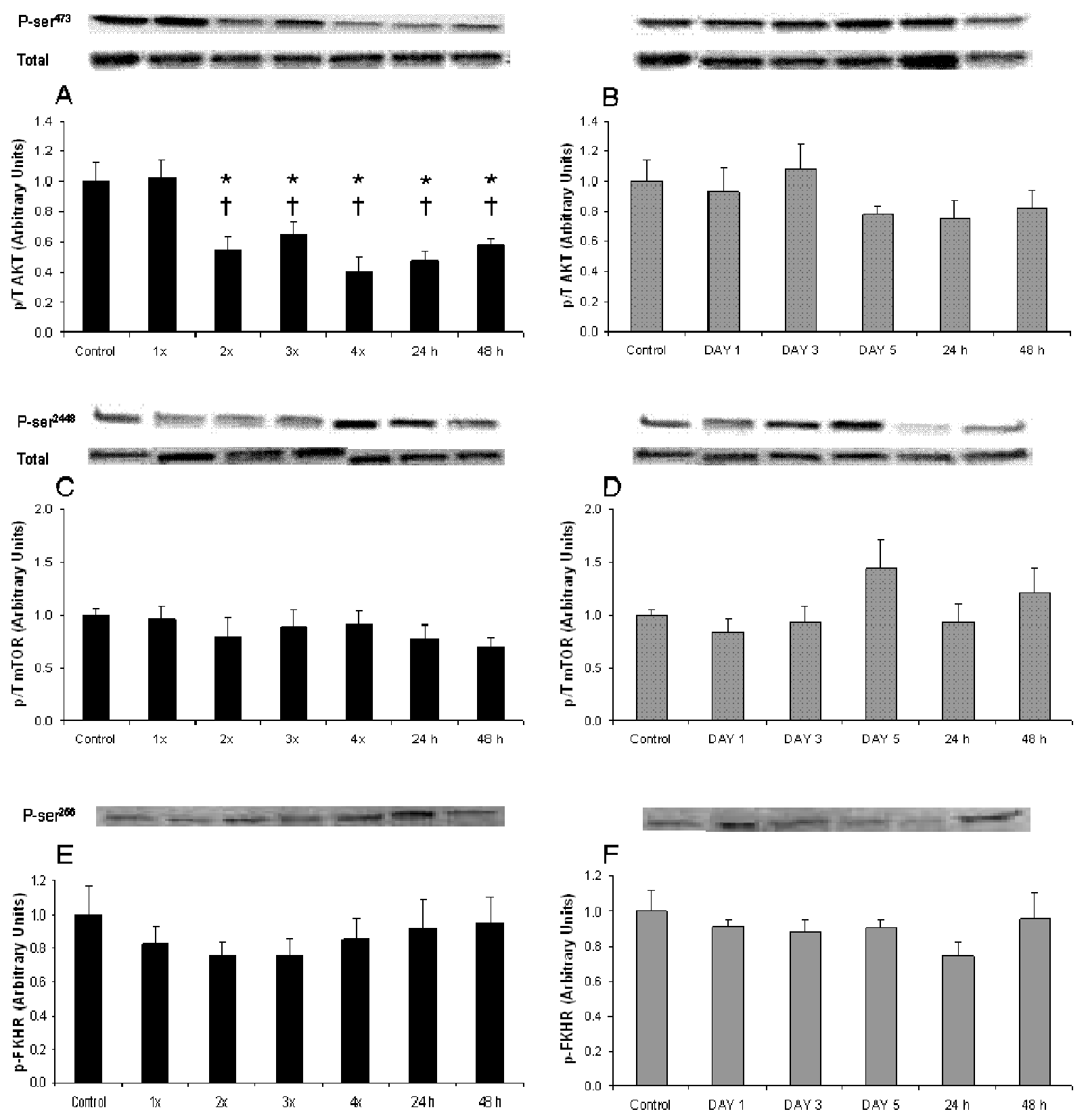

Figure 2.3 Phosphorylated $\mathrm{AKT}^{\mathrm{ser} 473}(\mathrm{~A}, \mathrm{~B})$ and mammalian target of rapamycin ${ }^{\text {ser2448 }}$ (mTOR; C, D) relative to total, and $\mathrm{FKHR}^{\mathrm{ser} 256}$ (FoxO1) phosphorylation (E, F) following either high-frequency "stacked" resistance training comprising four bouts of $3 \times 10$ repetitions separated by $3 \mathrm{~h}$ recovery (A, C, E; $\mathrm{n}=6$ per group) or conventional-frequency training consisting of three bouts of $4 \times 10$ repetitions with $48 \mathrm{~h}$ between resistance training bouts $(\mathrm{B}$, $\mathrm{D}, \mathrm{F} ; \mathrm{n}=6$ per group). Protocols were matched for total work and repetitions were performed at $75 \%$ one-repetition maximum with 3 min recovery between sets. White quadriceps muscle 
was extracted $3 \mathrm{~h}$ after every training bout, and 24 and $48 \mathrm{~h}$ following the final exercise session of each protocol, respectively. Results are group means ( \pm SE) and data are arbitrary values expressed relative to control. Significant difference (One-way ANOVA, P<0.05) vs. $*$ control; $\dagger 1 \times$.

p70 S6 Kinase (p70 S6K) / Eukaryotic Initiation Factor 4E-binding Protein (4E-BP1)

No difference in 4E-BP $1^{\text {thr70 }}$ phosphorylation was observed following HF and CF resistance training $(\sim 15-25 \%$, NS, Figure $2.4 \mathrm{~A}$ and $2.4 \mathrm{~B})$. In contrast, p70 S6K phosphorylation was increased significantly after both $\mathrm{HF}$ and CF resistance training. Phosphorylation of p70 S6K ${ }^{\text {thr389 }}$ was increased $3 \mathrm{~h}$ post-exercise with HF compared with control values, reaching significance after $3 \times$ and $4 \times$ bouts of training $(\sim 500 \%, \mathrm{P}<0.05$, Figure $2.4 \mathrm{C})$. Likewise, $\mathrm{p} 70$ S6K increased $3 \mathrm{~h}$ after CF training, an increase that was significantly different from control on DAY 1 and DAY 5 ( 500-700\%, P<0.05, Figure 2.4D). Phosphorylation of p70 S6K had returned to control levels 24 and $48 \mathrm{~h}$ after the final training bout for both training protocols.

\section{Cytokines and Mitogen Activated Protein Kinase (MAPK)}

HF resistance training resulted in increased expression of tumor necrosis factor alpha (TNF $\alpha)$ at all post-exercise time points $(\sim 13-54 \%)$ but was only significantly different from control 3 $\mathrm{h}$ after the fourth training bout $(\mathrm{P}<0.05$, Figure $2.5 \mathrm{~A})$. TNF $\alpha$ content significantly increased after DAY 1 of $\mathrm{CF}(\sim 38 \%, \mathrm{P}<0.05)$ but had returned to control values by DAY 5 (Figure 2.5B). Significant increases in $\mathrm{p} 38^{\text {thr180 }}$ MAPK phosphorylation were consistently observed after each bout with HF training and $24 \mathrm{~h}$ after the last exercise bout $(\sim 67-100 \%, \mathrm{P}<0.05$, Figure 2.5C), while CF training increased p38 compared to control on DAY 1 and DAY 3 $(\sim 65-118 \%, \mathrm{P}<0.05$, Figure $2.5 \mathrm{D})$. Inhibitor kappa B kinase alpha/beta $\left(\mathrm{IKK} \alpha / \beta^{\mathrm{ser} 180 / 181}\right)$ phosphorylation was significantly higher than control following $3 \mathrm{~h}$ recovery after all $\mathrm{HF}$ 
training bouts $(\sim 65-75 \%, \mathrm{P}<0.05$, Figure $2.5 \mathrm{E})$ but was only increased following exercise on DAY 1 with CF resistance training $(\sim 63 \%, \mathrm{P}<0.05$, Figure $2.5 \mathrm{~F})$.
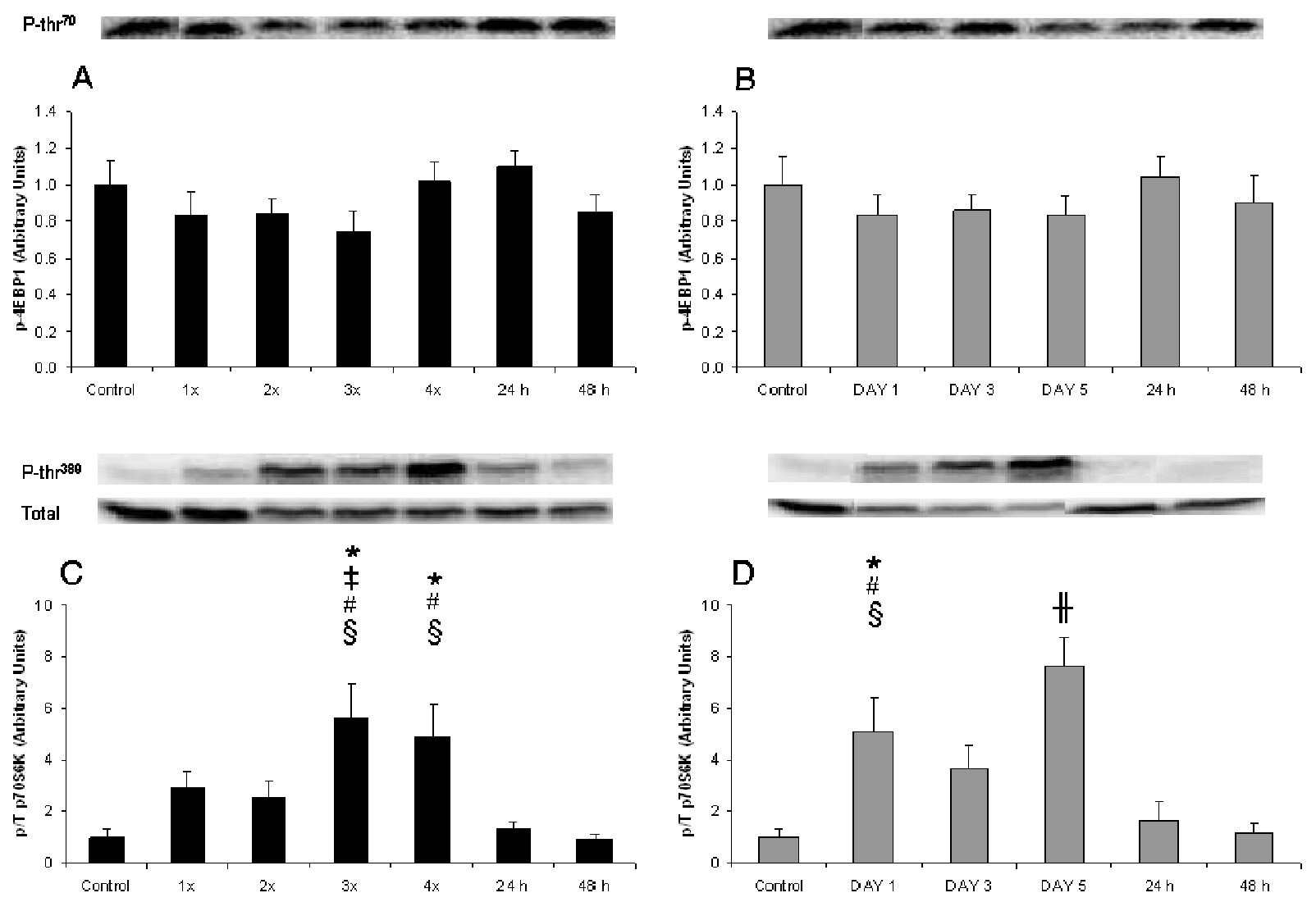

Figure 2.4 Eukaryotic initiation factor 4E-binding $\operatorname{protein}^{\text {thr70 }}$ (4E-BP1; A, B) phosphorylation and ribosomal p70 S6 kinase ${ }^{\text {thr389 }}$ (p70 S6K) phosphorylation relative to total (C, D) following either high-frequency "stacked" resistance training comprising four bouts of $3 \times 10$ repetitions separated by $3 \mathrm{~h}$ recovery $(\mathrm{A}, \mathrm{C} ; \mathrm{n}=6$ per group) or conventionalfrequency training consisting of three bouts of $4 \times 10$ repetitions with $48 \mathrm{~h}$ between resistance training bouts $(B, D ; n=6$ per group). Protocols were matched for total work and repetitions were performed at $75 \%$ one-repetition maximum with 3 min recovery between sets. White quadriceps muscle was extracted $3 \mathrm{~h}$ after every training bout, and 24 and $48 \mathrm{~h}$ following the final exercise session of each protocol, respectively. Results are group means $( \pm \mathrm{SE})$ and data are arbitrary values expressed relative to control. Significant difference (One-way ANOVA, $\mathrm{P}<0.05)$ vs. *control, $\$ 2 \times, \# 24 \mathrm{~h}, \S 48 \mathrm{~h}, \#$ all. 

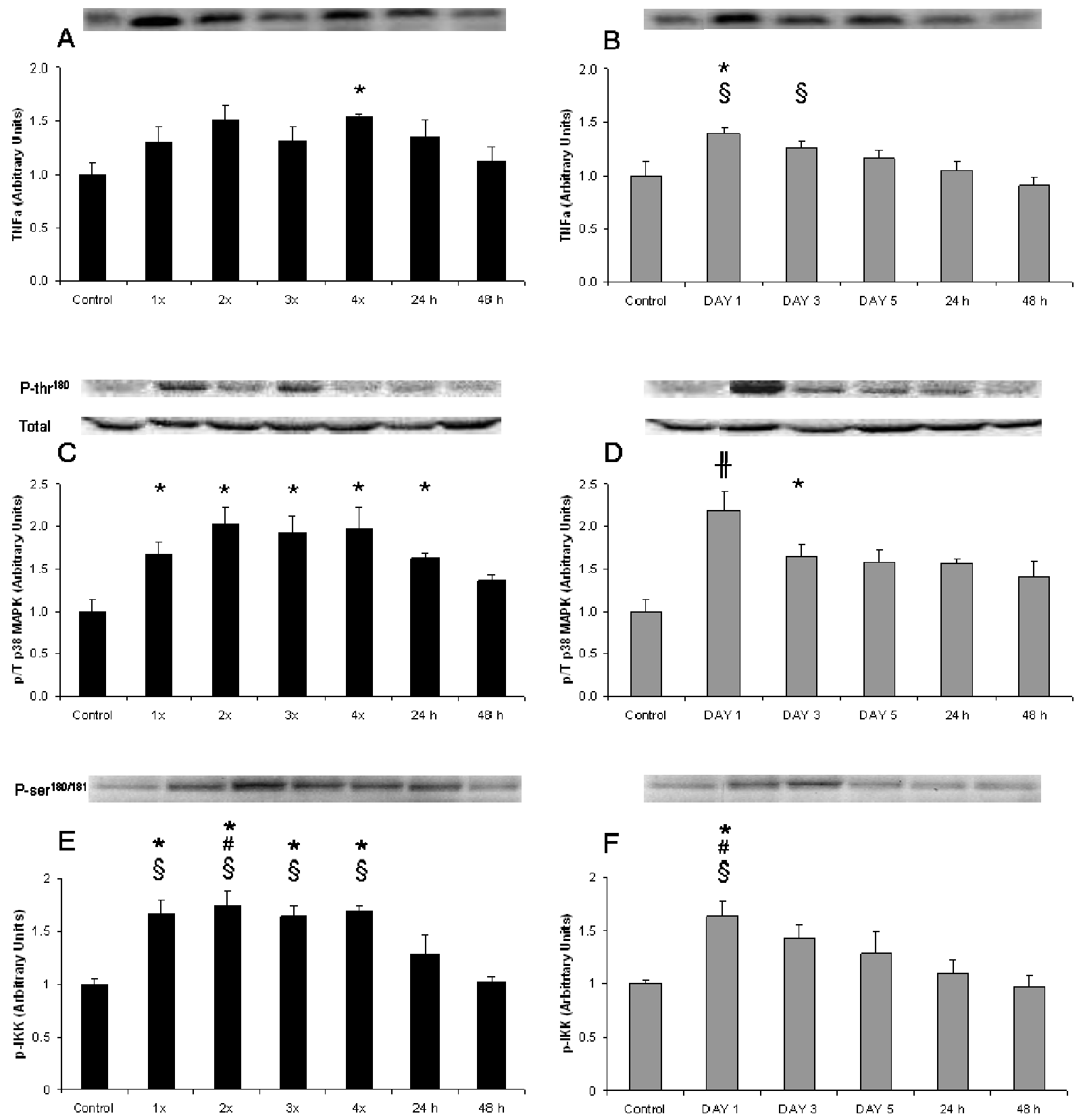

Figure 2.5 Total tumor necrosis factor alpha $(\mathrm{TNF} \alpha)$ content $(\mathrm{A}, \mathrm{B})$, phosphorylated $\mathrm{p} 38^{\text {thr180 }}$ mitogen activated protein kinase (MAPK) relative to total $(\mathrm{C}, \mathrm{D})$, and inhibitor kappa $\mathrm{B}$ kinase $^{\text {ser180/181 }}$ (IKK) phosphorylation (E, F) following either high-frequency "stacked" resistance training comprising four bouts of $3 \times 10$ repetitions separated by $3 \mathrm{~h}$ recovery (A, $\mathrm{C}, \mathrm{E} ; \mathrm{n}=6$ per group) or conventional-frequency training consisting of three bouts of $4 \times 10$ repetitions with $48 \mathrm{~h}$ between resistance training bouts $(\mathrm{B}, \mathrm{D}, \mathrm{F} ; \mathrm{n}=6$ per group). Protocols 
were matched for total work and repetitions were performed at $75 \%$ one-repetition maximum with 3 min recovery between sets. White quadriceps muscle was extracted $3 \mathrm{~h}$ after every training bout, and 24 and $48 \mathrm{~h}$ following the final exercise session of each protocol, respectively. Results are group means $( \pm \mathrm{SE})$ and data are arbitrary values expressed relative to control. Significant difference (One-way ANOVA, $\mathrm{P}<0.05$ ) vs. *control, \# $24 \mathrm{~h}, \S 48 \mathrm{~h}, \#$ all.

\subsection{Discussion}

The key components of any training programme are the volume, intensity and frequency of exercise sessions (Hawley 2002). The sum of these inputs can be termed the training stimulus that either enhances or impairs adaptation. Initially, training adaptations are directly related to the volume of work performed. However, the control mechanisms signalling adaptive responses are eventually titrated by exercise duration (Booth and Watson 1985) indicating that a maximal duration exists beyond which additional stimulus will not induce significant adaptation. If continually increasing exercise volume is insufficient for driving the adaptive response this suggests that the intensity and frequency of the overload stimulus are important for disruption to homeostasis and subsequent adaptation in skeletal muscle. The present study compared a conventional resistance training regimen with a novel "stacking" protocol in an attempt to identify the effects of training frequency on discrete cellular and molecular events following repetitive overload. It was hypothesised that compared to a conventional resistance training protocol, high-frequency training sessions would extend the transient response of key signalling pathways leading to an additive or "stacked" effect and a concomitant upregulation of signalling pathways involved in hypertrophy. In contrast to the original hypothesis, this study provides novel evidence to demonstrate that high-frequency overload suppressed AKT phosphorylation and exacerbated expression of TNF $\alpha$, IKK activity and p38 MAPK phosphorylation compared to a conventional training regimen. Nevertheless, both 
training protocols resulted in increases in $\mathrm{p} 70 \mathrm{~S} 6 \mathrm{~K}$ phosphorylation independent of the activation states of AKT and mTOR.

High-frequency training bouts resulted in a persistent suppression of muscle glycogen content, whereas the longer recovery time (and greater nutrient intake) between conventional training sessions was sufficient to replenish glycogen levels (Figure 2.2). Indeed, with the conventional training regimen there was moderate 'supercompensation' on DAY 3 whereby glycogen levels were increased above resting basal values. It has been proposed that AMPK acts as a "fuel gauge" in muscle cells, switching off ATP-consuming pathways when cellular energy is low, while concomitantly turning on alternative pathways for ATP regeneration. Accordingly, the suppressed glycogen content in the working muscles throughout the highfrequency training protocol was associated with a gradual increase in AMPK phosphorylation with each successive bout (Figure 2.2). In contrast, with longer recovery between training bouts and fuel status restored, $\mathrm{AMPK}^{\text {thr172 }}$ responses were blunted throughout the conventional-frequency training protocol.

AKT is a critical mediator of insulin / IGF signalling that when activated phosphorylates multiple substrates mediating important aspects of carbohydrate metabolism, and protein synthesis and degradation (Taniguchi et al. 2006). This study shows a systematic down-regulation in the phosphorylation state of AKT following repeated bouts of exercise, an effect that persisted for up to $48 \mathrm{~h}$ following multiple bouts undertaken in a single day (Figure 2.3). Given the proposed role for AKT in compensatory hypertrophy (Bodine et al. 2001b; Lai et al. 2004) the original hypothesis was that a cumulative increase in the phosphorylation of $\mathrm{AKT}^{\text {ser473 }}$ would occur following high-frequency resistance training. Nevertheless, AKT activity has previously been shown to both increase (Nader and Esser 2001; Bolster et al. 2003b; Sakamoto et al. 2004a; Creer et al. 2005) or remain unchanged (Widegren et al. 1998; Krisan et al. 2004) in response to a variety of contractile stimuli. There are a number of possible explanations for the dephosphorylation of AKT in response to stacked resistance 
training bouts. The most likely relates to the suppression and / or activation of kinases and phosphatases involved in the direct regulation of AKT activity. In this regard, Gao and colleagues (Gao et al. 2005) have shown that the $\mathrm{pH}$ domain leucine-rich repeat protein phosphatase (PHLPP) specifically controls the phosphorylation state of serine $e^{473}$ and catalyses its dephosphorylation in vivo significantly reducing AKT activity. Importantly, this dephosphorylation can lead to an increase in apoptosis and suppression of cell growth, effects that have yet to be confirmed in skeletal muscle (Gao et al. 2005). Nevertheless, the possibility exists that the demanding stimulus provided by stacked resistance training bouts resulted in PHLPP mediated down-regulation of AKT and increased apoptosis.

In addition, phosphorylation of the activation loops on threonine ${ }^{308}$ and the hydrophobic motif on serine ${ }^{473}$ is required for full activation of AKT (Alessi et al. 1997; Sarbassov et al. 2005b). Insulin receptor substrate-1 (IRS-1) phosphorylation and insulin signalling has been shown to be impaired by increases in TNF $\alpha$ (del Aguila et al. 1999; Plomgaard et al. 2005). In the current study a sustained and consistent increase in TNF $\alpha$ following high-frequency overload was observed. TNF $\alpha$ appears to exert its effect on IRS-1 by activation of IKK in a p38 MAPK-dependent manner (Del Aguila et al. 2000; Gao et al. 2002; de Alvaro et al. 2004). In support of this contention, the present findings show corresponding IKK and p38 phosphorylation concomitant with AKT dephosphorylation, indicating a possible TNF $\alpha$-mediated effect on AKT activity. Del Aguila and colleagues (Del Aguila et al. 2000) have previously reported that IRS-1 signalling and AKT kinase activation is impaired by muscle damage-induced TNF $\alpha$ production. Taken together, these findings indicate the muscle damage following high-frequency resistance training and subsequent increases in TNF $\alpha$ signalling may have exacerbated the persistent decrease in phosphorylation of AKT.

AKT is involved in the regulation of numerous cellular processes including protein synthesis and degradation (Nader 2005). AKT has been shown to mediate protein synthesis 
and degradation via the AKT-mTOR and AKT-FoxO1 pathways respectively (Nave et al. 1999; Sandri et al. 2004; Stitt et al. 2004; Latres et al. 2005), yet $\mathrm{mTOR}^{\mathrm{ser} 2448}$ and FoxO1 ${ }^{\mathrm{ser} 256}$ phosphorylation was largely unaffected by either resistance training regimen or changes in AKT phosphorylation (Figure 2.3). Two mTOR protein complexes exist where mTOR binds with a G-beta-L protein $(\mathrm{G} \beta \mathrm{L})$ and either a rapamycin-sensitive raptor or a rictor protein (Sarbassov et al. 2005b; Sarbassov et al. 2006). The mTOR-raptor complex appears to regulate cell growth through p70 S6K and 4E-BP1 while mTOR-rictor phosphorylates and activates $\mathrm{AKT}^{\mathrm{ser} 473}$ (Sarbassov et al. 2005a). While the mechanism(s) responsible for the regulation of the mTOR-rictor complex is currently unknown, a decrease in mTOR-rictor with high-frequency training may have contributed to the down-regulation of AKT phosphorylation. Furthermore, mTOR-raptor has been shown to be negatively regulated by the tuberous sclerosis complex 1/2 (TSC1/2)-Ras homologue enriched in brain (Rheb) pathway and AMPK appears to promote TSC2-Rheb mediated inhibition of mTOR-raptor activity (Garami et al. 2003; Williamson et al. 2006a). The increase in AMPK phosphorylation with the high-frequency training stimulus may have contributed to the lack of response in mTOR activity following this protocol. Indeed, Williamson and colleagues (Williamson et al. 2006a) have shown AMPK activation suppresses protein synthesis through repression of mTOR signalling. In the present study a modest decrease in phosphorylation of the FoxO1 transcription factor following resistance training was also observed (Figure 2.3). This suggests that increased transcriptional activity of atrophy proteins may occur after strenuous exercise-induced contraction. However, further work is required to establish the effect of in vivo contractile activity on FoxO1 activation as previous investigations have shown AKT regulation of FoxO1 and subsequent ubiquitin ligase activity in cell culture and transgenic animals (Sandri et al. 2004; Stitt et al. 2004).

Several p70 S6K substrates exist but essentially phosphorylated p70 S6K activates processes increasing translation efficiency and protein synthesis while 4E-BP1 is deactivated 
with phosphorylation inhibiting its role as a repressor of the translational machinery (Bodine et al. 2001b; Ruvinsky and Meyuhas 2006). A novel finding of the present study was the pronounced summation in p70 S6K phosphorylation with both "stacked" and conventional resistance training protocols that occurred independent of AKT or mTOR phosphorylation (Figure 2.4). Phosphorylation of p70 S6K and 4E-BP1 has been shown to be regulated by AKT (Bodine et al. 2001b; Lai et al. 2004) and mTOR (Burnett et al. 1998; Ali and Sabatini 2005) and evidence suggests these kinases may be fundamental for the control of cell size (Ohanna et al. 2005). It is difficult to reconcile the increased p70 S6K phosphorylation in the absence of the activation of its upstream kinases. However, these findings support those of others showing the activation of $\mathrm{p} 70 \mathrm{~S} 6 \mathrm{~K}$ with a resistance training-like stimulus in rodents and humans (Bodine et al. 2001b; Atherton et al. 2005; Cuthbertson et al. 2005). The decrease in 4E-BP1 phosphorylation in the present study suggests a lack of mTOR mediated downstream effects that would abolish its role in p70 S6K activation supporting the contention that p70 S6K phosphorylation may occur independent of AKT / mTOR under certain conditions (Hornberger et al. 2004; Song et al. 2005). PDK1 has been implicated as having a role in regulating p70 S6K activation, but it does not appear to phosphorylate p70 S6K ${ }^{\text {thr389 }}$ (Alessi et al. 1998; Pullen et al. 1998). Although evidence suggests that p70 S6K and 4E-BP1 are direct substrates of mTOR-raptor it cannot be ruled out that other unique kinases regulate p70 S6K / 4E-BP1 phosphorylation.

$\mathrm{TNF} \alpha$ is an inflammatory cytokine shown to negatively affect anabolic processes by increasing activity of protein degradation pathways, destabilising myogenic differentiation and altering transcriptional activity (Garcia-Martinez et al. 1994; Lang et al. 2002; Langen et al. 2004). Primary targets of $\mathrm{TNF} \alpha$ signalling include the inhibitor kappa $\mathrm{B}$ kinase (IKK) and p38 MAPK (Li et al. 2005; Chen et al. 2006). A fundamental role of IKK is phosphorylation of the nuclear factor kappa $\mathrm{B}(\mathrm{NF} \kappa \mathrm{B})$ inhibitor $(\mathrm{I} \kappa \mathrm{B})$ which designates $\mathrm{I} \kappa \mathrm{B}$ for ubiquitination and subsequent degradation, releasing the transcription factor NFאB to translocate to the 
nucleus and initiate the expression of a number of genes, including those involved in regulation of skeletal muscle protein degradation such as muscle ring finger 1 protein (MuRF1; Cai et al. 2004). Similarly, TNFa stimulated p38 phosphorylation also appears to induce gene expression of the E3 ubiquitin ligase muscle atrophy $\mathrm{F}$ box (MAFBx) protein promoting protein degradation in skeletal muscle (Li et al. 2005). The in vivo data from this study show that changes in IKK and p38 phosphorylation were highly coordinated with the responses of TNF $\alpha$ (Figure 2.5). Specifically, the repeated stimulus during the stacked training protocol induced a significant increase in IKK and p38 phosphorylation following each discrete exercise bout. In contrast, a single exercise bout increased phosphorylation of IKK and p38 after $3 \mathrm{~h}$ recovery on DAY 1 of conventional training, but this response was diminished with subsequent training bouts. The present findings are in agreement with recent work by Sriwijitkamol and colleagues (Sriwijitkamol et al. 2006) that showed reduced TNF $\alpha$ and increased $\mathrm{I} \kappa \mathrm{B}$ content in human skeletal muscle following an $8 \mathrm{wk}$ exercise programme. Taken together, these results suggest that chronic exercise is capable of promoting antiinflammatory effects that may be protective against stress-induced damage in skeletal muscle.

The initial and sustained increase in IKK / TNF $\alpha$ pathway signalling with highfrequency overload suggests that resistance exercise generates significant alterations in cellular homeostasis and a substantial pro-inflammatory response in skeletal muscle. Moreover, it is likely that the increases in IKK and TNF $\alpha$ in the present study were the result of muscle damage per se, as a subgroup of animals subjected to an acute bout of prolonged swimming showed no alterations in IKK phosphorylation or $\mathrm{I} \kappa \mathrm{B} \alpha$ degradation (data not shown).

In conclusion, this study compared a high-frequency resistance training protocol (matched for total work) with a conventional-frequency training regimen in order to elucidate the discrete signalling events following divergent overload stimuli. The data show that the frequency of overload is an important factor determining subsequent signalling events that 
underlie the adaptation processes. Specifically, this work provides novel evidence to demonstrate that high-frequency overload suppressed AKT phosphorylation and exacerbated the expression of $\mathrm{TNF} \alpha, \mathrm{IKK} \alpha / \beta$ activity and p38 MAPK phosphorylation. In contrast, conventional-frequency resistance training attenuated these pro-inflammatory signalling events. Both training protocols resulted in increases in p70 S6K phosphorylation independent of the activation states of AKT and mTOR. These data provide the first information on the time-course of events regarding the effects of exercise frequency on signal transduction pathways governing hypertrophy / atrophy regulatory responses in skeletal muscle in vivo. 


\section{Chapter Three}

Early signalling responses to divergent exercise stimuli in skeletal muscle from well-trained humans

Adapted from: Coffey V.G., Zhong Z., Shield A., Canny B.J., Chibalin A.V., Zierath J.R, Hawley J.A. The FASEB Journal 20(1): 190-2, 2006. 


\subsection{Introduction}

Conventional endurance and strength training exercise induce diverse adaptive responses. Endurance training elicits a variety of metabolic and morphological responses that function to minimise cellular disturbances during subsequent training sessions (Hawley 2002), including mitochondrial biogenesis (Holloszy 1967), a fast-to-slow twitch fibre phenotype transformation (Zierath and Hawley 2004), a decreased reliance on carbohydrate-based fuels during sub-maximal exercise (Holloszy et al. 1977) and improved whole-body aerobic capacity $\left(\mathrm{VO}_{2 \max } ;\right.$ Saltin 1969). In contrast, strength / resistance training has minimal effect on the muscle fibre phenotype (Williamson et al. 2001), $\mathrm{VO}_{2 \max }$ (Allen et al. 1976), or patterns of substrate utilisation during sub-maximal exercise. Strength / resistance training and some forms of high-intensity intermittent stimulation are associated with an increase in protein synthesis (Nader and Esser 2001; Rennie et al. 2004) and substantial muscle hypertrophy (Rennie and Tipton 2000) that promotes gains in maximal force output, whereas endurance training does not promote muscle hypertrophy or increase the force-output ability of muscle (Hickson 1980).

Although the molecular signalling mechanisms that transduce the effects of contractile activity to modify the skeletal muscle phenotype and function are incompletely understood, a number of pathways have been implicated. For example, activation of the 5' AMP-activated protein kinase (AMPK)- peroxisome proliferator activated receptor gamma co-activator-1 $\alpha$ (PGC-1 $\alpha$ ) signalling pathway may partly explain some of the adaptations to endurance training, while selective up-regulation of the AKT-tuberous sclerosis complex 2 (TSC2)mammalian target of rapamycin (mTOR) cascade may underlie the increase in skeletal muscle protein synthesis and growth observed after resistance training (Atherton et al. 2005). While the chronic, training-induced adaptations in skeletal muscle are the result of the cumulative effect of repeated bouts of exercise, the initial signalling responses that promote long-term adaptations are likely to occur after each training session (Widegren et al. 2001). Accordingly, 
this study investigated the early signalling events in skeletal muscle that are elicited in response to different types of contractile stimuli (e.g. endurance and strength training). Utilising a unique design in which athletes from different training backgrounds (i.e. chronically endurance- or strength-trained) undertook exercise in their habitual training mode, and "crossed over" to perform an acute bout in a non-familiar exercise discipline, the results are expected to characterise the acute signalling events underlying the specific adaptations to diverse modes of contractile activity associated with endurance and strength training.

\subsection{Methods}

\section{Materials}

Monoclonal anti-phospho-extracellular regulating kinase $1 / 2\left(\mathrm{ERK} 1 / 2^{\mathrm{Thr} 202 / \mathrm{Tyr} 204}\right)$, polyclonal anti-AKT $^{\text {Ser473/Thr308, }}$, were from New England BioLabs (Beverly, MA, U.S.A.). Polyclonal anti-p-Tuberin/TSC2 ${ }^{\text {Thr1462 }}$, Phospho-AMPK ${ }^{\text {Thr172 }}$, polyclonal anti-ribosomal protein S6 kinase $70 \mathrm{kDa}\left(\mathrm{p} 70 \mathrm{~S} 6 \mathrm{~K}^{\mathrm{Thr} 389}\right)$ kinase, polyclonal anti-phospho- eukaryotic translation initiation factor 2 beta $\left(\mathrm{eIF} 2 \mathrm{~B}^{\mathrm{Ser} 540}\right.$ ) and polyclonal anti-phospho-p38 ${ }^{\text {Tyr188/182 }}$ were from Cell Signaling Technology, Inc. (Beverly, MA, U.S.A.). Enhanced chemiluminescence reagents were from Amersham Biosciences (Buckinghamshire, U.K.). Other reagents are from Sigma Chemicals (St. Louis, MO, U.S.A.).

\section{Subjects}

Thirteen trained male volunteers participated in this investigation. Six subjects were cyclists who had been participating in regular endurance training (ET) for 8 yr. These subjects did not undertake any form of resistance training. The other 7 subjects were power-lifters who had been participating in regular strength / resistance training (ST) for $9 \mathrm{yr}$ and did not participate in any kind of endurance training. Characteristics of subjects from the two diverse training backgrounds are displayed in Table 3.1. The experimental procedures and possible risks 
associated with the study were explained to each subject, who gave written informed consent prior to participation. The study was approved by the Human Research Ethics Committee of RMIT University.

Table 3.1 Subject characteristics.

\begin{tabular}{lcc}
\cline { 2 - 3 } & $\begin{array}{c}\text { Endurance Trained } \\
(\mathrm{n}=6)\end{array}$ & $\begin{array}{c}\text { Strength Trained } \\
(\mathrm{n}=7)\end{array}$ \\
\hline Mass $(\mathrm{kg})$ & $74.7 \pm 7.6$ & $96.9 \pm 15.5^{*}$ \\
Age $(\mathrm{yr})$ & $28.7 \pm 6.1$ & $30.7 \pm 8.4$ \\
Training History $(\mathrm{yr})$ & $8.5 \pm 2.7$ & $9.0 \pm 7.3$ \\
$V O_{2 p e a k}\left(\mathrm{ml} \cdot \mathrm{kg}^{-1} \cdot \mathrm{min}^{-1}\right)$ & $65.2 \pm 6.4$ & $36.9 \pm 7.4^{*}$ \\
Maximum Strength $(\mathrm{N} \cdot \mathrm{m})$ & & $309 \pm 45^{*}$ \\
Concentric & $214 \pm 40$ & $389 \pm 60^{*}$ \\
Eccentric & $243 \pm 53$ & \\
\hline
\end{tabular}

All values are means \pm standard deviation. * Significant difference between endurance-trained and strength-trained groups (Students $t$-test, $\mathrm{P}<0.05$ ).

Overview of Study Design

The study consisted of a crossover design. All subjects performed two different exercisetesting protocols in randomised order: one experimental trial was undertaken in the subject's habitual training discipline, while the other trial was performed in a non-familiar exercise mode. 
Preliminary Tests

Peak Oxygen Uptake ( $\left.\mathrm{VO}_{2 p e a k}\right)$

Peak oxygen uptake $\left(\mathrm{VO}_{2 p e a k}\right)$ was determined during an incremental maximal cycling test to volitional fatigue on an isokinetic Lode bicycle ergometer (Groningen, The Netherlands). Briefly, the test protocol commenced at an intensity equivalent to $3.33 \mathrm{~W} \cdot \mathrm{kg}^{-1}$ or $2.5 \mathrm{~W} \cdot \mathrm{kg}^{-1}$ of body mass (BM) for endurance- and strength-trained subjects, respectively. This workload was maintained for $150 \mathrm{~s}$ then increased by $50 \mathrm{~W}$ for the second workload and $25 \mathrm{~W}$ every $150 \mathrm{~s}$ thereafter until subjects reached volitional fatigue, defined as the inability to maintain a cadence $>70 \mathrm{rev} \cdot \mathrm{min}^{-1}$. Throughout the maximal test breath-by-breath expired gas was passed

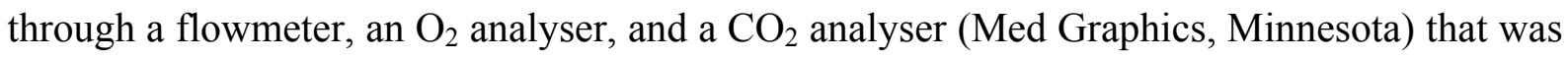
calibrated before testing using a 3-L Hans-Rudolph syringe and gases of known concentration $\left(4.00 \% \mathrm{CO}_{2}\right.$ and $\left.16.00 \% \mathrm{O}_{2}\right)$. The flowmeter and gas analysers were connected to a computer that calculated minute ventilation, oxygen uptake $\left(\mathrm{VO}_{2}\right), \mathrm{CO}_{2}$ production $\left(\mathrm{VCO}_{2}\right)$, and respiratory exchange ratio (RER) from conventional equations.

\section{Maximal Strength}

Maximal concentric and eccentric strength was determined using seated leg extensions, performed on a Kin-Com isokinetic dynamometer (Chattanooga, Tennessee, U.S.A.). The subject's right leg was strapped to the actuator arm immediately superior to the lateral malleolus of the lower leg with the lateral condyle of the femur visually aligned to the fulcrum of the actuator arm. Contractions were performed at $30^{\circ} \cdot \mathrm{s}^{-1}$ and subjects were instructed to extend their leg and resist the actuator arm with maximal effort during repetitions. Exercise range of motion was $85^{\circ}$, with leg extension endpoint set at $-5^{\circ}$ from full extension. Real time visual feedback was provided for all repetitions. Quadriceps strength was determined during a series of 3-repetition maximal leg extension sets with each set separated 
by $120 \mathrm{~s}$. Each individual $1-\mathrm{RM}$ was defined as the peak torque $(\mathrm{N} \cdot \mathrm{m})$ recorded during the concentric and eccentric contraction phases of the test protocol.

\section{Diet/Exercise Control}

Twenty-four hours before each exercise testing session (described subsequently), subjects refrained from vigorous physical activity and were provided with standardised pre-packed meals that consisted of $3 \mathrm{~g} \mathrm{CHO} \cdot \mathrm{kg}^{-1}$ body mass (BM), $0.5 \mathrm{~g}$ protein $\cdot \mathrm{kg}^{-1} \mathrm{BM}$, and $0.3 \mathrm{~g}$ fat $\cdot \mathrm{kg}^{-1} \mathrm{BM}$, to be consumed as the final intake the evening prior to reporting for an exercise testing session.

\section{Exercise Testing Session}

On the morning of a testing session subjects reported to the laboratory in a 10-12 h overnight fasted state. After resting quietly in a supine position for $10 \mathrm{~min}$ local anaesthesia (2-3 $\mathrm{ml}$ of $1 \%$ Xylocaine (lignocaine)) was administered to the skin, subcutaneous tissue and fascia of the vastus lateralis in preparation for muscle sampling. A resting biopsy was taken by using a $6 \mathrm{~mm}$ Bergstrom needle with suction applied (Evans et al. 1982). Approximately $100 \mathrm{mg}$ of skeletal muscle was removed and immediately frozen in liquid $\mathrm{N}_{2}$. At this time, separate sites on the same leg $(\sim 5 \mathrm{~cm}$ distal $)$ were prepared for subsequent sampling. Biopsies were taken from the left leg when subjects performed the cycling exercise session and the right leg for the resistance training session. Immediately upon completion of each exercise testing session, a second biopsy was taken and then subjects rested in a supine position for $3 \mathrm{~h}$. At the end of the $3 \mathrm{~h}$ recovery period, during which time the subjects were only allowed access to water, a third biopsy was taken. Every attempt was made to extract tissue from approximately the same depth in the muscle and to freeze the sample in liquid $\mathrm{N}_{2}$ within $10 \mathrm{~s}$. Samples were stored at $-80{ }^{\circ} \mathrm{C}$ until subsequent analysis. 
Cycling Exercise Session

Subjects performed 60 min of continuous cycling at a power output that elicited $\sim 70 \%$ of individual $\mathrm{VO}_{2 \text { peak }}$.

Resistance Exercise Session

Maximal voluntary isokinetic single leg extensions were performed on a Kin-Com dynamometer (as described previously). Subjects performed 8 sets of 5 repetitions at maximal intensity, separated by a 3 min recovery period. Peak and mean torque were recorded for each leg extension set.

Analytical Procedures

Total RNA Isolation and Reverse Transcription

Total RNA from $\sim 20 \mathrm{mg}$ of wet muscle was isolated using the ToTALLY RNA Kit (Ambion Inc., Austin, TX, U.S.A.) protocol and reagents. Total RNA concentration was determined spectrophotometrically at 260 and $280 \mathrm{~nm}$. RNA was reverse transcribed to synthesise firststrand cDNA using AMV reverse transcriptase (Promega, Madison, WI, U.S.A.) as described previously (Wadley et al. 2001). The cDNA was stored at $-20{ }^{\circ} \mathrm{C}$ for subsequent analysis.

\section{Primer Design and mRNA Quantification}

Peroxisome proliferator activated receptor gamma co-activator-1 $\alpha$ (PGC-1 $\alpha)$ primers were designed using Primer Express software version 2.0 (Applied Biosystems, Foster City, CA.) from GenBank sequence accession no. NM_013261. Primer specificity was confirmed using BLAST (Altschul et al. 1990). Primers were purchased from GeneWorks (Adelaide, SA, Australia). Primer efficiency (E) of each target amplification for a series of dilutions was calculated using $\mathrm{E}=\left(10^{-1 / \text { slope }}\right)-1$. Primers demonstrated efficient amplification. 
Real-time PCR was performed using the GeneAmp ${ }^{\circledR} 5700$ Sequence Detection System (Applied Biosystems). Reaction volumes $(20 \mu \mathrm{l})$ contained $2 \times$ SYBR Green PCR Master Mix (Applied Biosystems), forward and reverse primers and cDNA template (diluted 1:40). Samples were run in duplicate for 1 cycle $\left(50{ }^{\circ} \mathrm{C} 2 \mathrm{~min}, 95^{\circ} \mathrm{C} 10 \mathrm{~min}\right)$ followed by 40 cycles $\left(95{ }^{\circ} \mathrm{C} 15 \mathrm{~s}, 60{ }^{\circ} \mathrm{C} 60 \mathrm{~s}\right)$ and fluorescence emissions were measured after each cycle. A melting point dissociation curve was generated to confirm single product amplification. Expression of the gene of interest was calculated by subtracting the $\mathrm{C}_{\mathrm{T}}$ of the target gene for a given sample from the $\mathrm{C}_{\mathrm{T}}$ of the appropriate control sample. Expression of the gene of interest relative to control was calculated using the expression $2^{-\Delta \mathrm{CT}}$.

\section{Western Blot Analysis}

Skeletal muscle biopsies were freeze-dried overnight and dissected under a microscope to remove visible blood, fat and connective tissue. Skeletal muscles were homogenised in icecold homogenisation buffer containing $50 \mathrm{mM}$ HEPES, pH 7.5, $150 \mathrm{mM} \mathrm{NaCl}, 5 \mathrm{mM}$ EDTA, $10 \mathrm{mM}$ sodium pyrophosphate, $2 \mathrm{mM}$ sodium vanadate, and a protease inhibitor cocktail (1 $\mathrm{mM}$ phenylmethylsulfonyl fluoride, and $10 \mu \mathrm{g} / \mathrm{mL}$ each of aprotinin, leupeptin, and pepstatin). The lysate was kept on ice for 30 min and subjected to centrifugation at $12,000 \mathrm{~g}$ for $10 \mathrm{~min}$ at $4{ }^{\circ} \mathrm{C}$. Protein concentration was determined with a BCA Protein Assay (Pierce, Rockford, IL, U.S.A.). Aliquots of lysate (20 $\mu \mathrm{g}$ of protein) were re-suspended in Laemmli sample buffer. Proteins were then separated by SDS/PAGE, transferred to polyvinylidenedifluoride membranes (Millipore, MA), blocked with 7.5\% non-fat milk, washed with TBST (10 mM Tris $\mathrm{HCl}, 100 \mathrm{mM} \mathrm{NaCl}, 0.02 \%$ Tween 20) and incubated with appropriate primary antibodies overnight at $4{ }^{\circ} \mathrm{C}$. Membranes were washed with TBST and incubated with an appropriate secondary antibody. Both training groups were run on the same gel. Proteins were visualised by chemiluminescence and quantified by densitometry. The 
amount of phosphorylated proteins of the densitometric quantification is expressed as arbitrary units.

Statistical Analysis

Differences in subject characteristics and exercise performance were determined using twotailed $t$-test assuming unequal variances. Real-time PCR mRNA, and immunoblot phosphorylation and protein data are expressed in arbitrary units. Differences between means were determined by a one-way analysis of variance (ANOVA) with Newman-Keuls post-hoc test (GraphPad Prism version 3.0). All values are expressed as means and standard error (SE) with the critical level of significance established at $\mathrm{P}<0.05$.

\subsection{Results}

\section{Exercise Performance}

The average power output corresponding to $\sim 70 \%$ of $V O_{2 p e a k}$ for the 60 min cycling exercise session was $242 \pm 11$ vs. $168 \pm 10 \mathrm{~W}$ for endurance- and strength-trained subjects, respectively $(\mathrm{P}<0.001)$. Four strength-trained subjects were unable to complete the prescribed workload during the cycling bout. In these cases, power output was reduced to $60 \%$ of $\mathrm{VO}_{2 p e a k}$ after $30 \mathrm{~min}$ for the remainder of the prescribed exercise time. Peak force during the isokinetic resistance exercise session was $263 \pm 10$ vs. $190 \pm 4 \mathrm{~N}$ for strength- and endurancetrained subjects, respectively $(\mathrm{P}<0.001)$.

\section{Signalling Responses}

\section{$A M P K$}

Phosphorylation of AMPK increased immediately following cycling exercise in strengthtrained (54\%; P <0.05, Figure 3.1A), but not endurance-trained subjects. Conversely, AMPK phosphorylation was increased immediately post-exercise in endurance- $(114 \%$; $\mathrm{P}<0.05$, 
Figure 3.1B), but not strength-trained subjects after resistance exercise. Effects on AMPK phosphorylation were lost within $3 \mathrm{~h}$ recovery following both exercise modes.
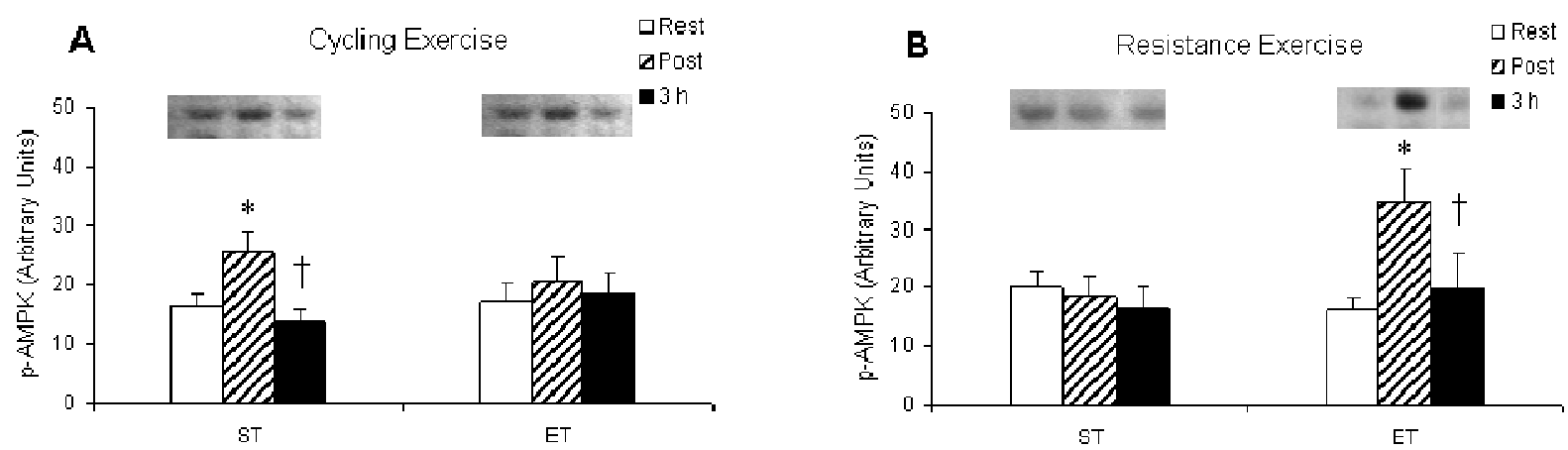

Figure 3.1 '5 adenosine monophosphate kinase (AMPK) phosphorylation in vastus lateralis muscle of strength-trained (ST; $n=7)$ and endurance-trained $(\mathrm{ET} ; \mathrm{n}=6)$ subjects in response to 60 min cycling at $\sim 70 \% V O_{2 p e a k}(\mathrm{~A})$ and $8 \times 5$ maximal isokinetic leg extensions (B). Values are group means $( \pm \mathrm{SE})$ at rest, immediately post-exercise (Post) and $3 \mathrm{~h}$ post-exercise (3 h). *Significant difference (One-way ANOVA) rest vs. post $(* \mathrm{P}<0.05)$; $\uparrow$ Significant difference post vs. $3 \mathrm{~h}(\uparrow \mathrm{P}<0.05)$.

\section{AKT-TSC2}

Following cycling exercise, $\mathrm{AKT}^{\mathrm{Ser} 473}$ phosphorylation was increased in endurance- $(50 \%$; $\mathrm{P}$ $<0.05$ ), but not strength-trained subjects (Figure 3.2A). The level of $\mathrm{AKT}^{\mathrm{Thr} 308}$ phosphorylation was unchanged (data not shown). After $3 \mathrm{~h}$ of recovery, AKT phosphorylation decreased below resting values in endurance-trained subjects $(67 \%$; $\mathrm{P}<0.01)$. AKT phosphorylation was similar between strength- and endurance-trained subjects following resistance exercise (Figure 3.2B). TSC2 phosphorylation was unaltered after cycle exercise in either strength- or endurance-trained subjects (Figure 3.2C). However, following resistance exercise, TSC2 phosphorylation was decreased in endurance-trained subjects immediately post-exercise $(47 \% ; \mathrm{P}<0.05)$, an effect that persisted for $3 \mathrm{~h}$ recovery $(40 \%$; $\mathrm{P}<0.05$, Figure 3.2D). 

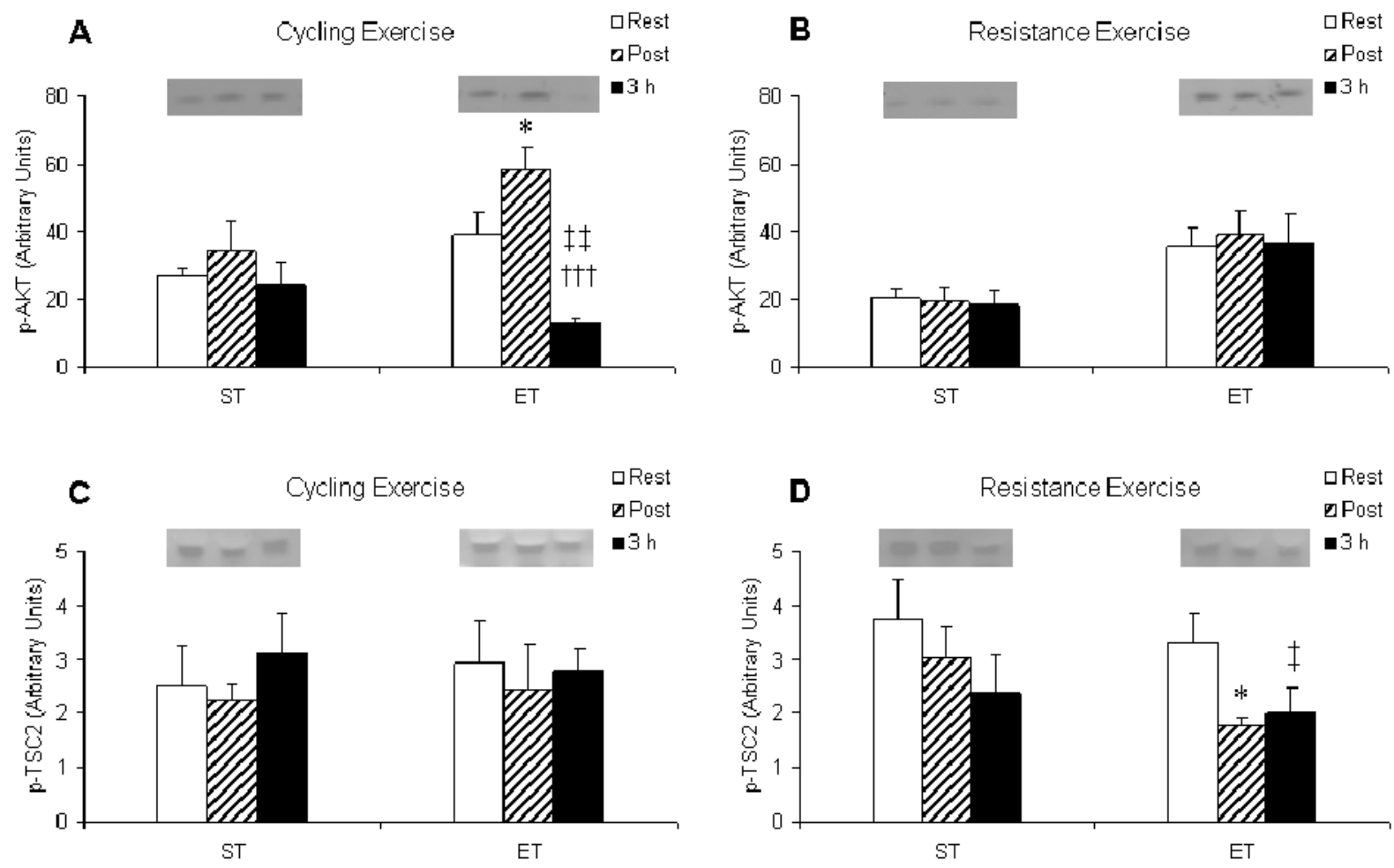

Figure 3.2 $\operatorname{AKT}(\mathrm{A}, \mathrm{B})$ and tuberous sclerosis complex-2 (TSC2; C, D) phosphorylation in vastus lateralis muscle of strength-trained $(\mathrm{ST} ; \mathrm{n}=7)$ and endurance-trained $(\mathrm{ET} ; \mathrm{n}=6)$ subjects in response to 60 min cycling at $\sim 70 \% V O_{2 p e a k}(\mathrm{~A}, \mathrm{C})$ and $8 \times 5$ maximal isokinetic leg extensions $(B, D)$. Values are group means $( \pm \mathrm{SE})$ at rest, immediately post-exercise (Post) and $3 \mathrm{~h}$ post-exercise $(3 \mathrm{~h})$. *Significant difference (One-way ANOVA) rest vs. post $(* \mathrm{P}<0.05)$; $₫$ Significant difference rest vs. $3 \mathrm{~h}(\ddagger \mathrm{P}<0.05, \pitchfork \uparrow \mathrm{P}<0.01) ; \uparrow$ Significant difference post vs. $3 \mathrm{~h}(\dagger \uparrow \uparrow \mathrm{P}<0.001)$. 

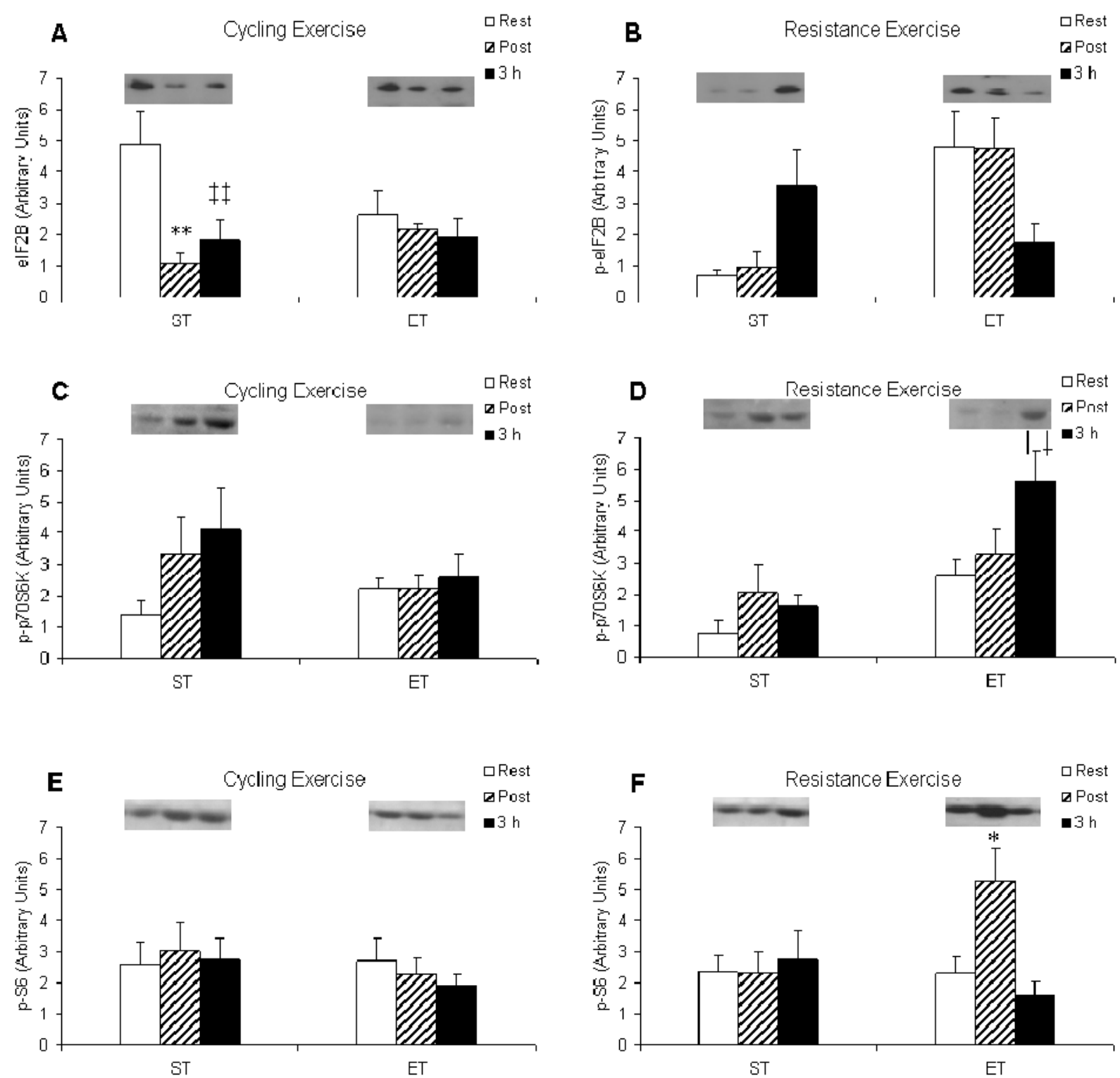

Figure 3.3 Eukaryotic initiation factor 2B (eIF2B; A, B) and ribosomal p70 S6 kinase (p70 $\mathrm{S} 6 \mathrm{~K} ; \mathrm{C}, \mathrm{D})$ phosphorylation, and ribosomal S6 protein abundance (S6; E, F) in vastus lateralis muscle of strength-trained (ST; $n=7)$ and endurance-trained (ET; $n=6)$ subjects in response to 60 min cycling at $\sim 70 \% V O_{2 p e a k}(\mathrm{~A}, \mathrm{C}, \mathrm{E})$ and $8 \times 5$ maximal isokinetic leg extensions $(\mathrm{B}$, $\mathrm{D}, \mathrm{F})$. Values are group means $( \pm \mathrm{SE})$ at rest, immediately post-exercise (Post) and $3 \mathrm{~h}$ postexercise $(3 \mathrm{~h}) .{ }^{*}$ Significant difference (One-way ANOVA) rest vs. post $(* \mathrm{P}<0.05, * * \mathrm{P}$ $<0.01)$; $\$$ Significant difference rest vs. $3 \mathrm{~h}(\$ \mathrm{P}<0.05, \$ \uparrow \mathrm{P}<0.01)$; $\dagger$ Significant difference post vs. $3 \mathrm{~h}(\dagger \mathrm{P}<0.05)$. 
eIF $2 B-p 70 S 6 K$

Cycling exercise induced a significant decrease in eIF2B phosphorylation in strength-trained ( $\mathrm{P}<0.01$, Figure $3.3 \mathrm{~A}$ ), but not endurance-trained subjects. eIF2B phosphorylation in strength-trained subjects was decreased immediately post-exercise $(78 \% \mathrm{P}<0.01)$ and this effect was maintained after 3 h recovery (63\%; P $<0.01$, Figure 3.3A). eIF2B phosphorylation was similar after resistance exercise in strength- and endurance-trained subjects following $3 \mathrm{~h}$ recovery (Figure 3.3B). Likewise, p70 S6K phosphorylation was similar between strengthand endurance-trained subjects after 60 min cycling exercise (Figure 3.3C). However, resistance exercise elicited a significant increase in $\mathrm{p} 70 \mathrm{~S} 6 \mathrm{~K}$ phosphorylation in endurancetrained subjects after $3 \mathrm{~h}$ of recovery $(118 \%$; $<<0.05$, Figure 3.3D). Phosphorylation of S6 protein, a substrate for $\mathrm{p} 70 \mathrm{~S} 6 \mathrm{~K}$, was increased immediately following resistance exercise in endurance-trained (129\%; $\mathrm{P}<0.05$; Figure 3.3F), but not strength-trained subjects.

\section{ERK $1 / 2-p 38$}

ERK phosphorylation after cycling or resistance exercise was similar between strength- and endurance-trained subjects (Figure 3.4A and 3.4B). p38 phosphorylation was increased in strength- $(74 \% ; \mathrm{P}<0.001)$, but not endurance-trained subjects immediately after cycling exercise (Figure 3.4C). In contrast, p38 phosphorylation was significantly increased in endurance-, but not strength-trained subjects after resistance exercise $(28 \%$; $\mathrm{P}<0.05$, Figure 3.4D). All exercise-induced phosphorylation effects were lost after $3 \mathrm{~h}$ recovery following both exercise modes. 

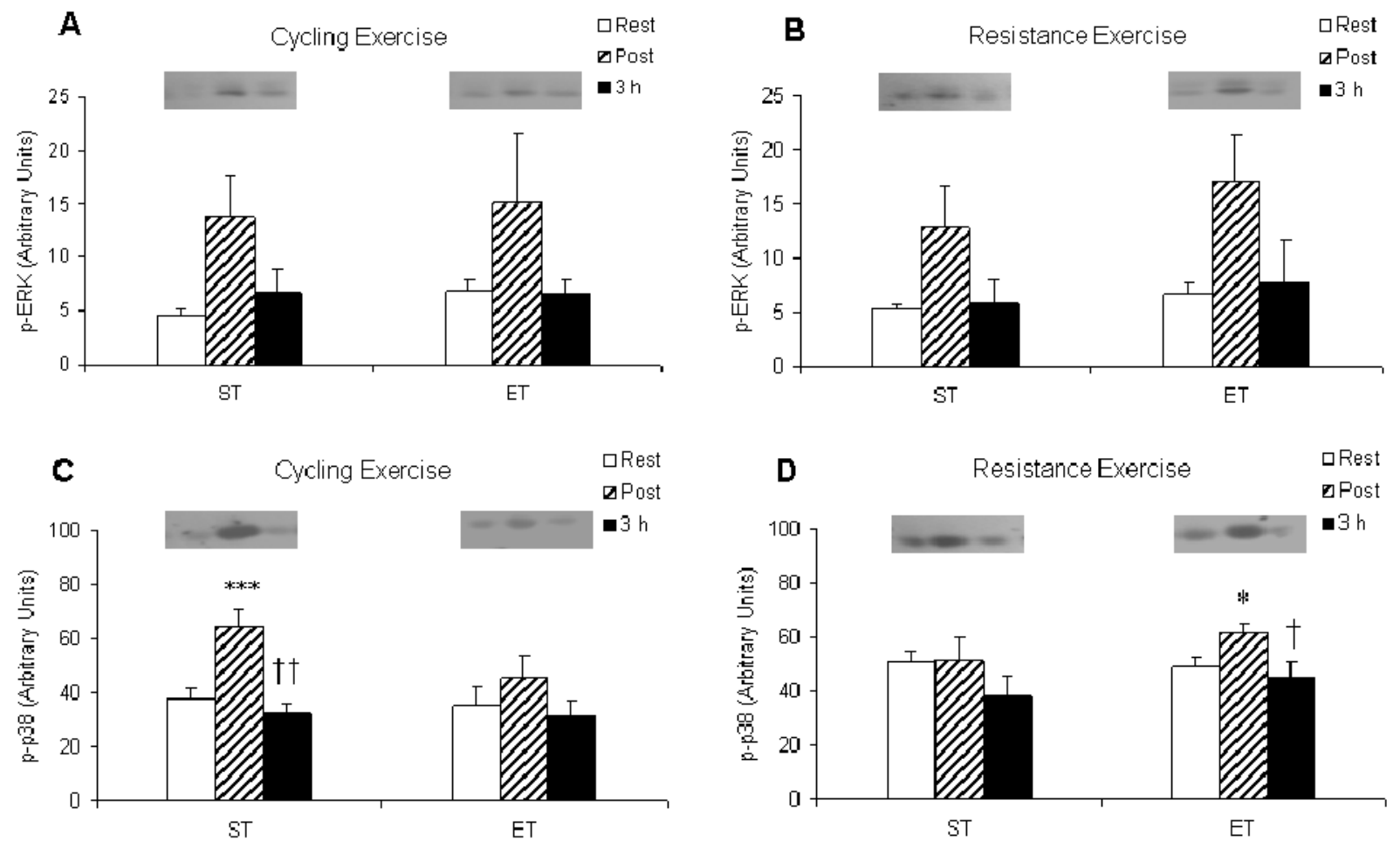

Figure 3.4 Extracellular regulating kinase (ERK; A, B) and p38 (C, D) MAPK phosphorylation in vastus lateralis muscle of strength-trained (ST; $n=7$ ) and endurancetrained $(\mathrm{ET} ; \mathrm{n}=6)$ subjects in response to 60 min cycling at $\sim 70 \% V O_{2 p e a k}(\mathrm{~A}, \mathrm{C})$ and $8 \times 5$ maximal isokinetic leg extensions $(B, D)$. Values are group means $( \pm \mathrm{SE})$ at rest, immediately post-exercise (Post) and $3 \mathrm{~h}$ post-exercise $(3 \mathrm{~h}) .{ }^{*}$ Significant difference (One-way ANOVA) rest vs. post $(* \mathrm{P}<0.05, * * * \mathrm{P}<0.001)$; $\uparrow$ Significant difference post vs. $3 \mathrm{~h}(\dagger \mathrm{P}<0.05, \dagger \dagger \mathrm{P}$ $<0.01)$.

\section{PGC-1 $\alpha$ mRNA and Protein Expression Responses to Exercise}

PGC-1 $\alpha$ mRNA was increased to a similar extent after cycling in both endurance-trained ( 8.5-fold, $\mathrm{P}<0.001)$ and strength-trained subjects $(\sim 10$-fold increase, $\mathrm{P}<0.001)$. However, there was little effect of resistance exercise on the mRNA expression of PGC- $1 \alpha$. PGC-1 $\alpha$ protein content after $3 \mathrm{~h}$ recovery from cycling or resistance exercise was unchanged in strength- and endurance-trained subjects (data not shown). 


\subsection{Discussion}

Contractile activity associated with physical exercise stimulates multiple signalling events and induces transient changes in gene transcription (Widegren et al. 2001; Sakamoto and Goodyear 2002). These acute alterations in cellular homeostasis may be responsible for the chronic adaptations that occur in skeletal muscle in response to repeated bouts of exercise (i.e. training). The disparity between the adaptations observed after chronic resistance or endurance training suggests these two types of contractile stimuli induce differential signalling and gene regulatory responses (Booth and Thomason 1991). To test this hypothesis, the current study utilised a unique design in which habitually strength-trained and endurancetrained athletes undertook an acute bout of exercise in each of these training modes in order to characterise the specific signalling events associated with diverse modes of contraction. The results provide evidence for the highly specialised nature of training adaptation and demonstrate that a degree of "response plasticity" capable of diverse adaptive compliance is conserved at opposite ends of the endurance-hypertrophy adaptation continuum.

AMPK is involved in the regulation of numerous adaptive responses including mitochondrial biogenesis, muscle hypertrophy and protein synthesis (Aschenbach et al. 2004). In response to exercise, isoform-specific and intensity-dependent changes in AMPK activity have been observed in human skeletal muscle (Yu et al. 2003). AMPK has been implicated as part of a metabolic 'switch' that, in concert with PGC-1 $\alpha$ (Terada et al. 2002; Jorgensen et al. 2005), promotes mitochondrial biogenesis and an 'endurance-training like' profile (Atherton et al. 2005). AMPK phosphorylation was increased in skeletal muscle from strength-trained subjects when non-habitual cycling exercise was performed and from endurance-trained subjects who undertook non-habitual resistance exercise (Figure 3.1). These changes in AMPK phosphorylation in human skeletal muscle in vivo contrast with the recent results in rodent skeletal muscle (Atherton et al. 2005), whereby signalling responses were determined in response to in vitro electrical stimulation. Compared to rest, low-frequency electrical 
stimulation to mimic endurance training increased $\mathrm{AMPK}^{\mathrm{Thr} 172}$ phosphorylation (relative to total AMPK), while high-frequency electrical stimulation (to mimic resistance training) decreased AMPK phosphorylation (Atherton et al. 2005).

There are a number of possible explanations for the discordant findings between these studies. The most obvious relates to the use of different loads and stimulation patterns to induce muscle contraction. In vitro electrical stimulation represents an extreme stimulus, likely to exceed any stress that would be encountered by human skeletal muscle in vivo. However, an alternative explanation might be that [AMP] only increased sufficiently to activate AMPK when subjects performed exercise in their unfamiliar discipline (i.e. when endurance-trained subjects undertook resistance exercise, or when strength-trained individuals undertook sub-maximal cycling). In support of this contention, all strength-trained subjects reported experiencing extreme difficulty completing $60 \mathrm{~min}$ of continuous cycling at the prescribed power output, whereas all endurance-trained individuals coped with the demands of this exercise task effortlessly. If, in the case of the strength-trained subjects, part of the cycling exercise bout was undertaken at an intensity above the individual lactate threshold, then aerobic and glycolytic ATP resynthesis rates would be insufficient to maintain steadystate [phosphocreatine] and the concentrations of AMP would rise. Indeed, $\mathrm{Yu}$ and coworkers (2003) and Neilsen and colleagues (2003a) have previously shown that during cycling exercise performed at the same relative intensity, activation of several signalling intermediates (including AMPK) was greater in skeletal muscle from untrained subjects than from highly-trained cyclists with a prolonged history of endurance training, probably due to a better maintenance of the energy charge and a less pronounced exercise-induced acidification in exercise-trained muscle.

With regard to the increase in AMPK phosphorylation in endurance-trained subjects following resistance exercise, the recovery between maximal work-bouts was possibly too short to allow for complete resynthesis of [phosphocreatine]. This would lead to an increase in 
[AMP] to a level that is sufficient for AMPK activation. Finally, as AMPK has a glycogen binding domain (Hudson et al. 2003) and activity of AMPK is greater when skeletal muscle [glycogen] is low (Wojtaszewski et al. 2003), the possibility exists that greater net glycogen depletion occurred when subjects performed the unfamiliar exercise bout, compared to the habitual discipline. Unfortunately, adequate muscle sample was unavailable for analyses of glycogen and metabolite concentrations.

Along with AMPK, activation of PGC-1 $\alpha$ has recently been proposed to partially mediate specific adaptations to endurance training (Atherton et al. 2005). Indeed, evidence for increased expression of PGC-1 $\alpha$ in rodent skeletal muscle 12-18 h after a single bout of prolonged swimming exercise (Baar et al. 2002) and within $3 \mathrm{~h}$ of low frequency electrical stimulation in vitro (Atherton et al. 2005) has been provided, suggesting that increases in PGC-1 $\alpha$ protein expression represents a key regulatory component of the stimulation of mitochondrial biogenesis by exercise. In the present study, cycling resulted in marked increases in PGC-1 $\alpha$ mRNA in endurance- and strength-trained subjects, whereas resistance exercise had little effect on the mRNA expression. While endurance exercise undoubtedly enhances PGC-1 $\alpha$ mRNA expression, possibly through an AMPK-related mechanism (Terada et al. 2002), further work is required to directly link AMPK-PGC-1 $\alpha$ signalling with chronic adaptations to exercise.

AKT is a critical signalling mediator of cellular growth and metabolism in skeletal muscle (Glass 2003). AKT directly phosphorylates TSC2, activating mTOR, in part by deactivating TSC2 (Inoki et al. 2002). AKT can also directly phosphorylate (Nave et al. 1999) and activate mTOR (Scott et al. 1998) and phosphorylation of both AKT and mTOR are increased during skeletal muscle hypertrophy (Reynolds et al. 2002). Inhibition of mTOR activity blocks compensatory skeletal muscle hypertrophy in vivo (Bodine et al. 2001b), while ablation or inhibition of p70 S6K results in decreases in cell size (Montagne et al. 1999). Eukaryotic initiation factor eIF2 and its "exchange factor" eIF2B also play key roles in the 
regulation of protein synthesis and cell growth: phosphorylation of eIF2 inhibits eIF2B and thus translation initiation. While phosphorylation of eIF2 serves to impair protein synthesis, it causes up-regulation of the translation of specific mRNAs that encode transcription factors, therefore exerting effects on gene expression at multiple levels.

In agreement with others (Thorell et al. 1999; Sakamoto et al. 2004a), an increase in $\mathrm{AKT}^{\text {Ser473 }}$ phosphorylation was observed following cycling exercise in endurance-trained subjects. However, $\mathrm{AKT}^{\mathrm{Thr} 308}$ phosphorylation was unchanged. At present, there is little data on the response of AKT after an acute bout of resistance exercise undertaken by strengthtrained subjects, although a recent report provides evidence for increased AKT phosphorylation in endurance-trained cyclists after moderate-intensity resistance exercise (Creer et al. 2005). Interestingly, the increase in AKT phosphorylation in that study was observed only when subjects commenced exercise with high $(\sim 600 \mathrm{mmol} / \mathrm{kg}$ dry mass $)$ skeletal muscle glycogen content and not when pre-exercise glycogen levels were low ( 200 mmol/kg dry mass). Phosphorylation of AKT deactivates GSK3, which under basal conditions is thought to promote activation of glycogen synthase (Cross et al. 1995) and inhibit eIF2B activity (Vyas et al. 2002). The current results show a moderate increase in eIF2B phosphorylation in skeletal muscle $3 \mathrm{~h}$ after strength-trained subjects performed resistance exercise, and a concomitant decrease in phosphorylation after these subjects undertook endurance exercise. These findings are perplexing and suggest that strength trained subjects activated a prolonged translation initiation following endurance exercise, although the mechanism underlying this effect remains unknown.

The involvement of p70 S6K in skeletal muscle hypertrophy has been implicated from a variety of work / force overload models(Baar et al. 1999; Bodine et al. 2001b; Nader and Esser 2001; Rommel et al. 2001). Phosphorylation of p70 S6K and subsequent activation of ribosomal protein S6 enhances translation of mRNAs encoding elongation factors and ribosomal proteins, thereby increasing translational capacity (Kubica et al. 2005). Resistance 
exercise in endurance- but not strength-trained subjects elicited a significant increase in p70 S6K phosphorylation after $3 \mathrm{~h}$ recovery (Figure 3.3D) and in ribosomal $\mathrm{S} 6$ protein phosphorylation immediately post-exercise (Figure 3.3F). This finding is in agreement with previous work in humans (Karlsson et al. 2004), which showed that p70 S6K phosphorylation was increased following resistance training in healthy untrained men $2 \mathrm{~h}$ post-exercise when branch chain amino acids were administered. p70 S6K phosphorylation is also increased with high-frequency electrical stimulation and resistance training to induce cell growth and hypertrophy in rodents (Baar et al. 1999; Nader and Esser 2001; Kubica et al. 2005). An explanation for the lack of change in p70 S6K and S6 protein in the strength-trained subjects following resistance exercise is that these proteins are already sufficiently up-regulated to accommodate any further strength gains that may occur after subsequent bouts of resistance exercise (i.e. negative feedback). This theory may also explain why phosphorylation of $\mathrm{p} 70$ $\mathrm{S} 6 \mathrm{~K}$, a translational regulator, was only increased in endurance-trained subjects $3 \mathrm{~h}$ following resistance exercise. The increase in p70 S6K phosphorylation is consistent with the observation that phosphorylation of this protein and subsequent protein synthesis in skeletal muscle are only elevated in the long-term recovery period after an acute bout of resistance exercise undertaken by untrained subjects (Karlsson et al. 2004) and after in vitro electrical stimulation (Baar et al. 1999; Nader and Esser 2001). These models are representative of skeletal muscle with large potential for plasticity to adapt (i.e. the malleability of structure and function has yet to be determined).

The specific responses of mitogen activated protein kinases (MAPK) to different forms of exercise / contraction are incompletely understood. Indeed, previous investigations have established variable MAPK signalling responses with altered metabolic and mechanical stress, contraction modes, and training status following a variety of exercise stimuli (Widegren et al. 1998; Wretman et al. 2001; Nielsen et al. 2003a; Thompson et al. 2003; Yu et al. 2003). In accordance with some (Nader and Esser 2001), but in contrast with other 
studies (Wretman et al. 2001), findings from the current study provide no evidence for exercise-specific or training-specific effects on either ERK1/2 and p38 phosphorylation (Figure 3.4). The ERK1/2 pathway has been proposed to play a role in the formation of slow muscle fibres and to inhibit the formation of fast muscle fibres (Murgia et al. 2000; Higginson et al. 2002). While both forms of contraction were associated with a transient increase in ERK phosphorylation immediately post-exercise and a return to resting values after $3 \mathrm{~h}$ of recovery, these changes failed to reach statistical significance. Both low- and high-frequency electrical stimulation of rodent skeletal muscle leads to increased ERK1/2 phosphorylation (Atherton et al. 2005), suggesting that this pathway is involved in a general response to contractile activity. With regard to the responses of the p38 MAPK pathway to different exercise modalities, when subjects undertook exercise in their non-familiar activity, p38 MAPK phosphorylation was increased. Contraction-induced phosphorylation of p38 MAPK is induced by high mechanical stress, which might partly explain the increase in phosphorylation after endurance-trained subjects performed heavy resistance exercise (i.e. mechanical loading to which the muscle was unaccustomed or poorly adapted) (Wretman et al. 2001). However, in rodent skeletal muscle, p38 MAPK phosphorylation was unaltered in response to either lowor high-frequency electrical stimulation (Atherton et al. 2005). Data from this study supports the contention that $\mathrm{p} 38 \mathrm{MAPK}$ is not always phosphorylated in response to contractile activity (Wretman et al. 2001; Atherton et al. 2005).

In conclusion, linking specific signalling cascades to specialised metabolic and gene regulatory responses that occur in response to different modes of exercise is complicated by the fact that signalling pathways are non-linear and often constitute a complex and incompletely resolved network with a high degree of cross-talk, feedback regulation, transient activation and redundancy (Zierath and Hawley 2004). While information pertaining to the subsets of genes and putative signalling pathways activated in response to different modes of contraction in humans is emerging ( $\mathrm{Yu}$ et al. 2003; Yang et al. 2005), the mechanism for 
adaptive changes in skeletal muscle in response to training is incompletely resolved. Moreover, whether prior contractile activity (i.e. training history) affects the acute responses to divergent exercise stimuli is unknown. The results from the current study provide evidence that chronic endurance or strength training attenuates some of the exercise specific signalling responses involved in single mode adaptations to training. Although there is a possibility that pre-existing (genetic) differences between the two groups may also contribute to the acute exercise responses, this study demonstrates that human skeletal muscle retains the capacity to respond to divergent contractile stimuli and that a degree of "response plasticity" is conserved at opposite ends of the endurance-hypertrophy adaptation continuum. Although selective activation of the AMPK-PGC-1 $\alpha$ or AKT-TSC2-mTOR signalling pathways has been proposed to explain many of the specific adaptive responses to endurance or resistance training during in vitro electrically stimulated muscle contractions (Atherton et al. 2005), the in vivo findings from this study provide little evidence for the putative AMPK-AKT switch. 


\section{Chapter Four}

Interaction of contractile activity and training history on mRNA abundance in skeletal muscle from trained athletes

Adapted from: Coffey V.G., Shield A., Canny B.J., Carey K.A., Cameron-Smith D., Hawley J.A. American Journal of Physiology Endocrinology and Metabolism 290(5): E849-855, 2006. 


\subsection{Introduction}

Skeletal muscle displays an enormous plasticity to respond to contractile activity and loading conditions, with muscle from strength- and endurance-trained athletes representing diverse states of the "adaptation continuum." Endurance training of sufficient volume and intensity results in an increased whole-body maximal oxygen uptake and shifts in substrate utilisation from carbohydrate- to lipid-based fuels, largely as a result of an expanded mitochondrial density and volume (Irrcher et al. 2003). Such changes are brought about by the coordinated co-expression of both the nuclear and mitochondrial genomes that, together, ensure proper assembly and expansion of the mitochondrial reticulum (Adhihetty et al. 2003). Thus, endurance training-induced adaptations culminate in mitochondrial biogenesis and an organelle capable of improved ATP provision (Irrcher et al. 2003) and a concomitant enhancement of endurance capacity (Holloszy and Coyle 1984). In contrast, resistance exercise, comprising high-intensity, low volume loading, results in increased cross-sectional area of the trained musculature, mainly due to an increase in muscle contractile protein (Flück and Hoppeler 2003). Modulation of transcription and translation events contribute to changes in gene expression and subsequent protein synthesis in response to this mode of training (Bolster et al. 2003a). Accordingly, resistance training-induced adaptations that culminate in muscle hypertrophy are the result of integrated gene responses and coordinated molecular events that support the enlargement of pre-existing muscle cells via the incorporation of additional myonuclei (Flück and Hoppeler 2003).

Training adaptation can merely be viewed as the accumulation of specific proteins (Hansen et al. 2005). Hence, the altered gene expression that allows for these changes in protein concentration is of major importance for any subsequent training adaptation. The chronic adaptations to any training regimen are likely to be the result of the cumulative effects of repeated bouts of exercise (Sanders Williams and Neufer 1996; Pilegaard et al. 2000), with the initial cellular responses that lead to these long-term adaptations occurring after each 
(acute) training session (Widegren et al. 2001). Previous works have demonstrated the importance of a wide range of gene expression consistent with their central role in defining the adaptive phenotype with differing modes of training (Teran-Garcia et al. 2005; Vissing et al. 2005). However, for many genes the transcriptional activation and translational responses occur during the first few hours of recovery, returning to basal levels within $24 \mathrm{~h}$ of the exercise stimulus (Neufer and Dohm 1993; Pilegaard et al. 2000). Although much data is available describing the cellular and molecular mechanisms underlying the transcriptional regulation of gene expression after exercise the effects of prior training on the acute response to different types of contraction has not been investigated. Accordingly, the present study was undertaken to determine the early gene responses after an acute bout of endurance and resistance training. Utilising a design in which chronically endurance- or strength-trained athletes undertook exercise in their customary training mode and "crossed over" to perform an acute bout of exercise in their non-familiar discipline, this study should provide novel data on subsets of metabolic and myogenic genes that are likely to be fundamental to the specific exercise-induced adaptation in skeletal muscle.

\subsection{Methods}

\section{Subjects}

Thirteen trained male volunteers participated in this investigation. Six subjects were cyclists who had been participating in regular endurance training (ET) for an average of $8 \pm 3 \mathrm{yr}$. These subjects were not undertaking any form of resistance training, nor had they performed such training during the past 2 yr. The other 7 subjects were power-lifters who had been participating in regular strength / resistance training (ST) for $9 \pm 7 \mathrm{yr}$ and had not participated in any kind of endurance training for the past $2 \mathrm{yr}$. The characteristics of the two training populations are the same as those described in Chapter Two (page 98). The experimental procedures and possible risks associated with the study were explained to each subject who 
gave written informed consent prior to participation. The study was approved by the Human Research Ethics Committees of RMIT University.

\section{Overview of Study Design}

The study consisted of a crossover design. All subjects performed two different exercisetraining protocols to enable within subject comparisons. One experimental trial was undertaken in the subject's habitual training discipline, while the other trial was performed in their non-familiar exercise mode. Exercise testing sessions were separated by a minimum of $10 \mathrm{~d}$.

Preliminary Tests

Peak Oxygen Uptake (VO $\left.{ }_{2 p e a k}\right)$

Peak oxygen uptake $\left(\mathrm{VO}_{2 \text { peak }}\right)$ was determined during an incremental maximal cycling test to volitional fatigue on an isokinetic Lode bicycle ergometer (Groningen, Netherlands) as described in detail previously (Hawley and Noakes 1992).

\section{Maximal Strength}

Maximal concentric and eccentric strength was determined using seated leg extensions performed on a Kin-Com isokinetic dynamometer (Chattanooga, Tennessee). The subject's right leg was strapped to the actuator arm immediately superior to the lateral malleolus of the lower leg with the lateral condyle of the femur visually aligned to the fulcrum of the actuator arm. Contractions were performed at $30^{\circ} \cdot \mathrm{s}^{-1}$ and subjects were instructed to extend their leg and resist the actuator arm with maximal effort during concentric and eccentric leg extension repetitions, respectively. Exercise range of motion was $85^{\circ}$ with leg extension endpoint set at $-5^{\circ}$ from full extension. Quadriceps strength was determined during a series of $3 \times 3-$ repetition leg extensions with each set separated by $120 \mathrm{~s}$. Each individual 1-RM was defined 
as the peak torque $(\mathrm{N} \cdot \mathrm{m})$ recorded during the concentric and eccentric contraction phases of the test protocol.

\section{Diet/Exercise Control}

Before each exercise testing session subjects were required to refrain from vigorous physical activity for a minimum of $24 \mathrm{~h}$ and were provided with standardised pre-packed meals that consisted of $3 \mathrm{~g} \mathrm{CHO} \cdot \mathrm{kg}^{-1}$ body mass (BM), $0.5 \mathrm{~g}$ protein $\cdot \mathrm{kg}^{-1} \mathrm{BM}$, and $0.3 \mathrm{~g}$ fat $\cdot \mathrm{kg}^{-1} \mathrm{BM}$ to be consumed as the final caloric intake the evening prior to reporting to the laboratory.

\section{Exercise Testing Session}

On the morning of a testing session subjects reported to the laboratory in a 10-12 $\mathrm{h}$ overnight fasted state. After resting for $10 \mathrm{~min}$ local anaesthesia (2-3 $\mathrm{ml}$ of $1 \%$ Xylocaine (lignocaine)) was administered to the skin, subcutaneous tissue and fascia of the vastus lateralis in preparation for muscle biopsies. A resting biopsy $(\sim 100 \mathrm{mg})$ was taken by using a $6 \mathrm{~mm}$ Bergstrom needle with suction applied (Evans et al. 1982), removed and immediately frozen in liquid $\mathrm{N}_{2}$. At this time a separate site on the same leg $(\sim 5 \mathrm{~cm}$ distal) was prepared for a second biopsy. Biopsies were taken from the left leg when performing the cycling exercise session and the right leg for the resistance training session. Upon completion of each exercise testing session subjects rested in a supine position for $3 \mathrm{~h}$ and during this time water was consumed ad libitum. At the end of the recovery period a second muscle biopsy was taken. Every attempt was made to extract tissue from approximately the same depth in the muscle and to immediately freeze the sample in liquid $\mathrm{N}_{2}$. Samples were stored at $-80{ }^{\circ} \mathrm{C}$ until subsequent analysis. 


\section{Cycling Exercise Session}

Subjects performed 60 min of continuous cycling at a power output that elicited $\sim 70 \%$ of individual $\mathrm{VO}_{2 \text { peak }}$.

\section{Resistance Exercise Session}

Subjects performed maximal concentric and eccentric leg extensor contractions with their right leg on a Kin-Com dynamometer (as described previously). Subjects performed 8 sets of 5 maximal effort repetitions with 3 min recovery between sets. Peak and mean torque were recorded for each set.

Analytical Procedures

Total RNA Isolation and Reverse Transcription

Total RNA from $\sim 20 \mathrm{mg}$ of wet muscle was isolated using the ToTALLY RNA Kit (Ambion Inc., Austin, TX) protocol and reagents. Total RNA concentration was determined spectrophotometrically at 260 and $280 \mathrm{~nm}$. RNA was reverse transcribed to synthesize firststrand cDNA using AMV reverse transcriptase (Promega, Madison, WI). Extracted RNA (1 $\mu \mathrm{g}$ ) was heated at $65{ }^{\circ} \mathrm{C}$ for 10 min immediately prior to first strand cDNA being generated using AMV reverse transcriptase (kit A3500; Promega, Madison, WI) with Oligo(dT)15 primer, in the presence of $1 \mathrm{mM}$ of each $\mathrm{dNTP}$ and $20 \mathrm{U}$ of recombinant RNasin ribonuclease inhibitor. The reaction was incubated at $42{ }^{\circ} \mathrm{C}$ for $60 \mathrm{~min}$ and then terminated at $99{ }^{\circ} \mathrm{C}$ for 10 min and $4{ }^{\circ} \mathrm{C}$ for $5 \mathrm{~min}$. The cDNA was stored at $-20{ }^{\circ} \mathrm{C}$ for subsequent analysis. Reverse transcription was performed for all samples simultaneously. Previous work from this laboratory has demonstrated minimal variation in efficiency when performed under these conditions (Murphy et al. 2003). 


\section{Primer Design and mRNA Quantification}

PCR primers were designed using Primer Express software version 2.0 (Applied Biosystems, Foster City, CA.) from gene sequences obtained from GenBank (Table 4.2). Primer specificity was confirmed using BLAST (Altschul et al. 1990). Primers were purchased from GeneWorks (Adelaide, SA, Australia). Efficiency of PCR primers was confirmed by examining the dynamic range of responses for a series of dilutions of cDNA. Using the slopes of the lines, the efficiency (E) of each target amplification was calculated using the equation $\mathrm{E}$ $=(10-1 /$ slope $)-1$. All primers used in this study demonstrated efficient amplification.

Real-time PCR was performed using the GeneAmp ${ }^{\circledR} 5700$ Sequence Detection System (Applied Biosystems). For the PCR step, reaction volumes of $20 \mu \mathrm{l}$ contained $2 \times$ SYBR Green PCR Master Mix (Applied Biosystems), forward and reverse primers and cDNA template (diluted 1:40). All samples were run in duplicate. The real-time PCR reaction was run for 1 cycle $\left(50{ }^{\circ} \mathrm{C} 2 \mathrm{~min}, 95{ }^{\circ} \mathrm{C} 10 \mathrm{~min}\right)$ followed by 40 cycles $\left(95{ }^{\circ} \mathrm{C} 15 \mathrm{~s}, 60{ }^{\circ} \mathrm{C} 60 \mathrm{~s}\right)$ and fluorescence emissions was measured after each of the repetitive cycles. Because SYBR green indiscriminately binds to double-stranded DNA, a melting point dissociation curve was generated to confirm that only a single product was amplified. Heat dissociation of oligonucleotides detects differences in melting temperature and produces a single dissociation peak for each nucleotide within a $2{ }^{\circ} \mathrm{C}$ difference in melting temperature (Ririe et al. 1997). The selection of endogenous control to normalise for input RNA (housekeeping gene) was confounded by training history (strength or endurance trained) and / or exercise mode (cycling or resistance training) which generated significant variance in all of the commonly used exercise housekeeping genes ( $\beta$-actin, $\beta$ 2-microglobulin, GAPDH; Jemiolo and Trappe 2004; Mahoney et al. 2004). The expression of the gene of interest in a given sample was calculated by subtracting the $\mathrm{CT}$ of the target gene for a given sample from the $\mathrm{CT}$ of the same gene from the appropriate control sample, for that individual. The relative expression of the gene of interest relative to control was then calculated using the expression $2-^{\Delta \mathrm{CT}}$. 
Table 4.2 Primer sequences and concentrations used for real-time PCR.

\begin{tabular}{lrll}
\hline Gene & GenBank & Forward Primer $\left(5^{\prime} \rightarrow 3^{\prime}\right)$ & Reverse Primer $\left(5^{\prime} \rightarrow 3^{\prime}\right)$ \\
& Number & & \\
\hline MAFBx & NM_058229 & CATCCTTATGTACACTGGTCCAAAGA & TCCGATACACCCACATGTTAATG \\
MyoD & NM_002478 & CCGCCTGAGCAAAGTAAATGA & GCAACCGCTGGTTTGGAT \\
Myogenin & NM_002479 & GGTGCCCAGCGAATGC & TGATGCTGTCCACGATGGA \\
Myostatin & NM_005259 & CCAGGAGAAGATGGGCTGAA & CAAGACCAAAATCCCTTCTGGAT \\
PGC-1 $\alpha$ & NM_013261 & CAAGCCAAACCAACAACTTTATCTCT & CACACTTAAGGTGCGTTCAATAGTC \\
PDK4 & NM_002612 & ATGGATAATTCCCGGAATGCT & ACTTGGCATACAGACGAGAAATTG \\
VEGF & NM_003376.3 & GCGCAAGAAATCCCGGTATA & GCTTTCTCCGCTGAGCAA \\
\hline
\end{tabular}

PGC-1 $\alpha$, peroxisome proliferator activated receptor gamma co-activator $1 \alpha$; PDK4, pyruvate dehydrogenase kinase 4; VEGF, vascular endothelial growth factor; MAFBx, muscle atrophy F-box protein.

Statistical Analysis

Differences in subject characteristics and exercise performance were determined using twotailed t-test assuming unequal variances. Data for mRNA abundance with each exercise session are expressed in relative units after individual samples were normalised relative to mean values at rest. Data were subjected to repeated measures ANOVA and logtransformation was performed when significant deviations from homogeneity occurred (SPSS for Windows Version 12.0.1). Differences between individual means were compared using Fisher's LSD test. All values are expressed as means and standard deviation (SD) with the critical level of significance established at $\mathrm{P}<0.05$. 


\subsection{Results}

\section{Exercise Performance}

The power output corresponding to $\sim 70 \% \mathrm{VO}_{2 \text { peak }}$ for the 60 min cycling bout was $242 \pm 11$ vs. $168 \pm 10 \mathrm{~W}$ for ET and ST subjects, respectively $(\mathrm{P}<0.001)$. The mean peak force during the isokinetic resistance exercise session was $263 \pm 10$ vs. $190 \pm 4 \mathrm{~N}$ for ST and ET subjects, respectively $(\mathrm{P}<0.001)$.

\section{Changes in mRNA Abundance after Cycling Exercise}

Relative changes in mRNA abundance of metabolic genes in muscle sampled $3 \mathrm{~h}$ following 60 min of cycling exercise were observed for both ST and ET subjects (Figure 4.1A, 1B). Peroxisome proliferator activated receptor gamma co-activator $1 \alpha(\mathrm{PGC}-1 \alpha)$ mRNA was increased to a similar extent after cycling in both ET $(\sim 8.5$-fold, P $<0.001)$ and ST subjects $(\sim 10$-fold increase, $\mathrm{P}<0.001)$. There were also large increases in pyruvate dehydrogenase kinase 4 (PDK4) mRNA in both groups of subjects after cycling (ET $\sim 26$-fold, P $<0.05$; ST $\sim 39$-fold, $\mathrm{P}<0.05$ ). Likewise, the increase in vascular endothelial growth factor (VEGF) mRNA was similar in ET ( $\sim 4.5$-fold, $\mathrm{P}<0.01)$ and ST subjects ( $\sim$-fold, $\mathrm{P}<0.01)$ in response to cycling exercise.

Cycling exercise produced diverse responses between ET and ST subjects for the myogenic regulatory factors (MRF's) MyoD and Myogenin (Figure 4.2A, 2B). There was an increase in both MyoD ( 3-fold, $\mathrm{P}<0.05)$ and Myogenin mRNA $(\sim 0.9$-fold, $\mathrm{P}<0.05)$ in ET subjects after cycling. In contrast, cycling decreased MyoD ( 0.4-fold: NS) and Myogenin ( 0.4-fold: NS) in ST subjects. Muscle atrophy F-box protein (MAFBx) mRNA abundance was increased in both subject groups following cycling (ET $\sim 2$-fold, P $<0.01$; ST $\sim 0.4$-fold, P $<0.01)$ while Myostatin mRNA was significantly increased by cycling exercise in ET ( 2fold, $\mathrm{P}<0.05$ ) but not ST subjects. 
There was little effect of resistance exercise on the mRNA abundance of PGC-1 $\alpha$ or VEGF (Figure 4.3A, 3B). In contrast, PDK4 was significantly increased in ET $(\sim 7$-fold, $\mathrm{P}<0.01)$ but not ST subjects after resistance exercise. There were divergent responses of genes associated with myogenic regulation in ET subjects in response to resistance exercise (Figure 4.4A). In ET subjects, MyoD increased ( 0.7-fold: NS) but MAFBx ( $\sim 0.7$-fold: NS) and myostatin ( 0.6-fold: NS) both decreased after resistance exercise. In ST subjects, the mRNA abundance of the myogenic genes under investigation was not significantly altered by a single bout of resistance exercise (Figure 4.4B). 

A

$\begin{array}{ll}\text { A ET Cycling } & \square \text { Rest } \\ & \square 3 \mathrm{~h}\end{array}$

$\begin{array}{ll}\text { ET Cycling } & \square \text { Rest } \\ & \square 3 \mathrm{~h}\end{array}$

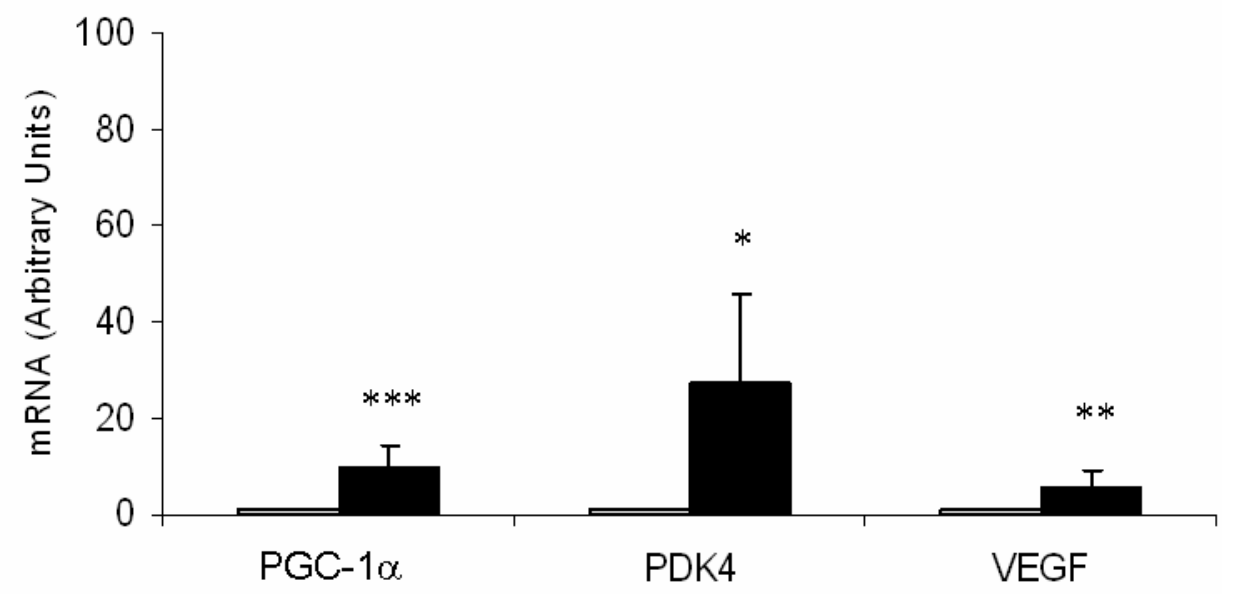

$\mathrm{B}$

ST Cycling

$\square$ Rest

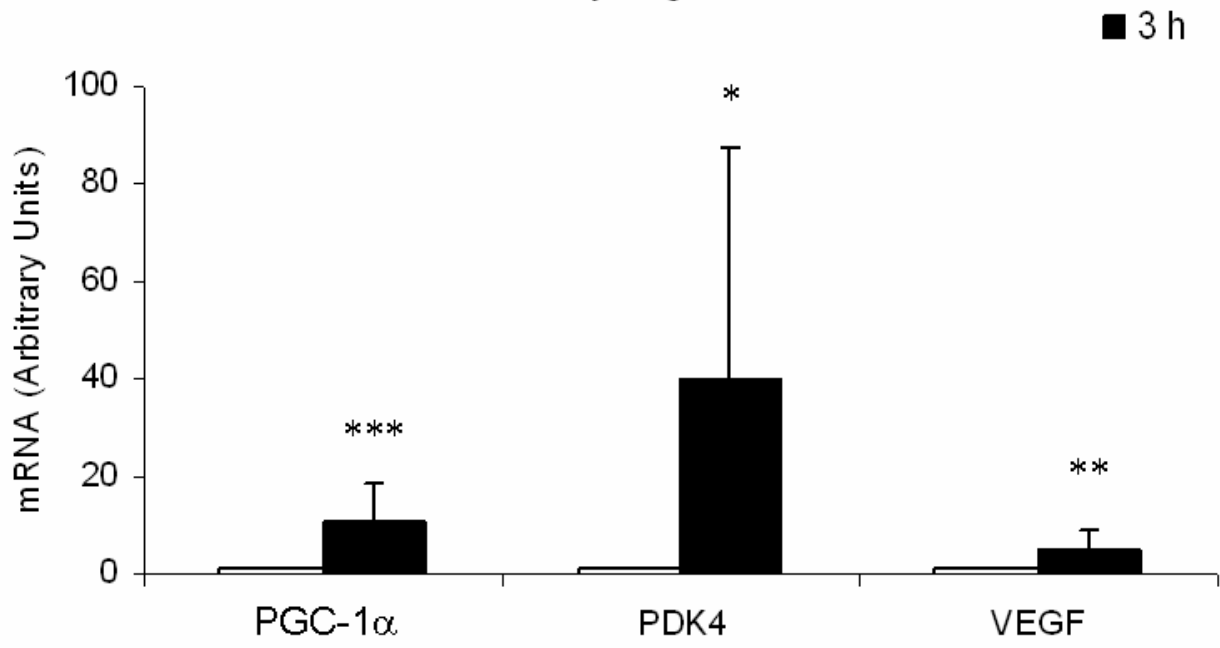

Figure 4.1 Changes in mRNA abundance for genes associated with endurance responses in (A) endurance-trained (ET; $n=6)$ and $(B)$ strength-trained $(\mathrm{ST} ; \mathrm{n}=7)$ subject vastus lateralis muscle sampled $3 \mathrm{~h}$ after 60 min cycling at $\sim 70 \% V O_{2 p e a k}$. All values are mean $\pm \mathrm{SD}$. PGC-1 $\alpha$, peroxisome proliferator activated receptor gamma co-activator $1 \alpha$; PDK4, pyruvate dehydrogenase kinase 4; VEGF, vascular endothelial growth factor. * Significant difference from rest (Repeated measures ANOVA, $* \mathrm{P}<0.05, * * \mathrm{P}<0.01, * * * \mathrm{P}<0.001$ ). 
A ET Cycling $\quad$ Rest

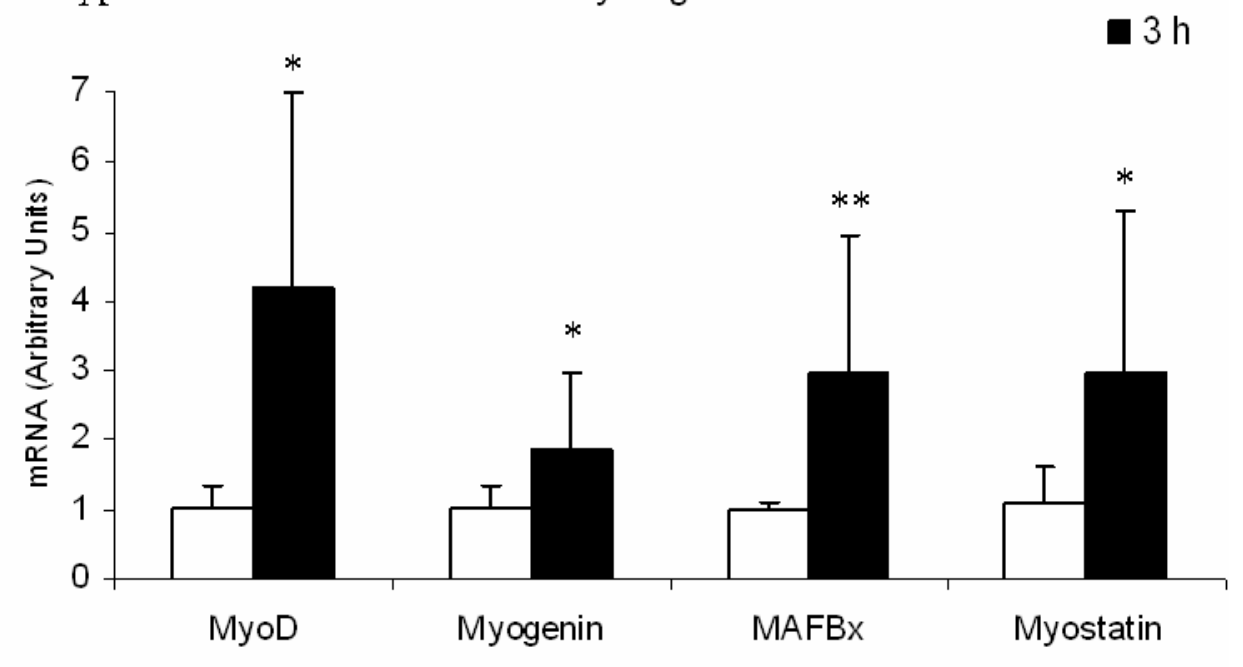

B ST Cycling $\quad \square$ Rest

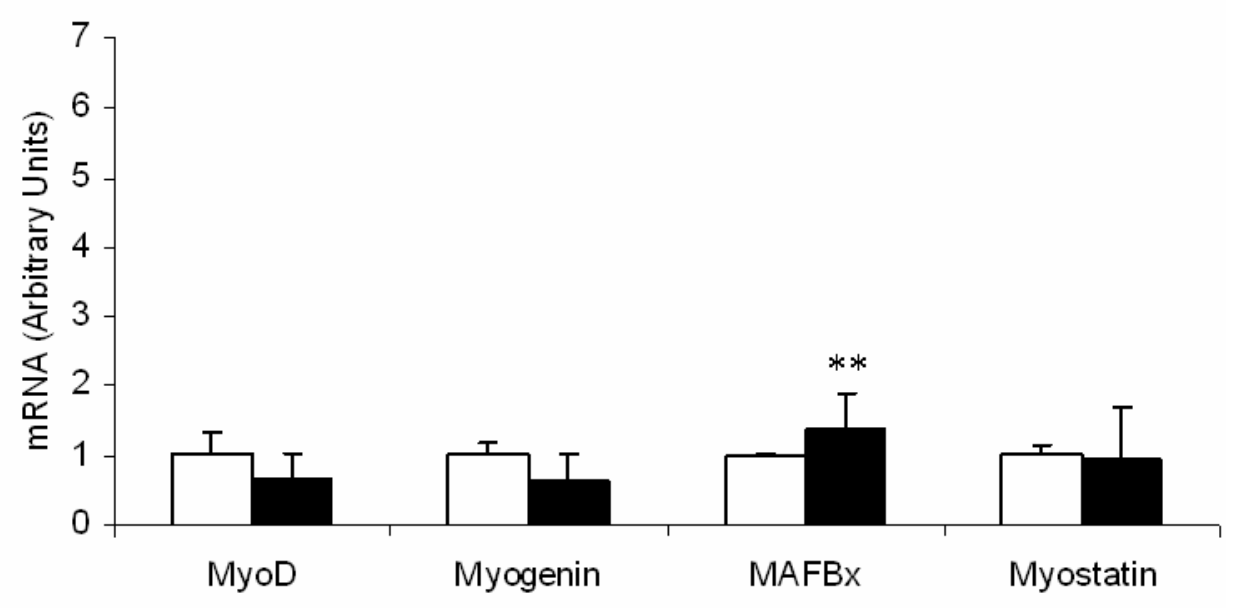

Figure 4.2 Changes in mRNA abundance for genes associated with myogenic responses in (A) endurance-trained (ET; $n=6)$ and (B) strength-trained (ST; $n=7)$ subject vastus lateralis muscle sampled $3 \mathrm{~h}$ after 60 min cycling at $\sim 70 \% V O_{2 p e a k}$. All values are mean $\pm \mathrm{SD}$. MAFBx, muscle atrophy F-box protein. * Significant difference from rest (Repeated measures ANOVA, $* \mathrm{P}<0.05, * * \mathrm{P}<0.01)$. 

A
ET Resistance
$\square$ Rest

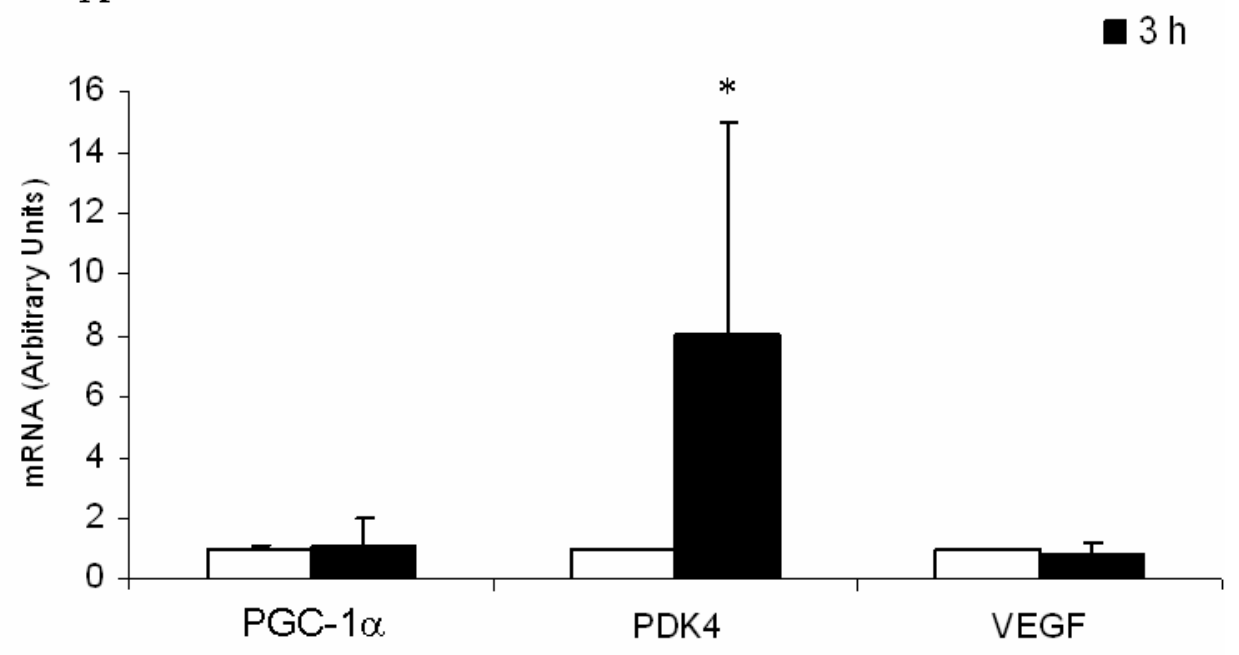

$\mathrm{B}$

ST Resistance

$\square$ Rest

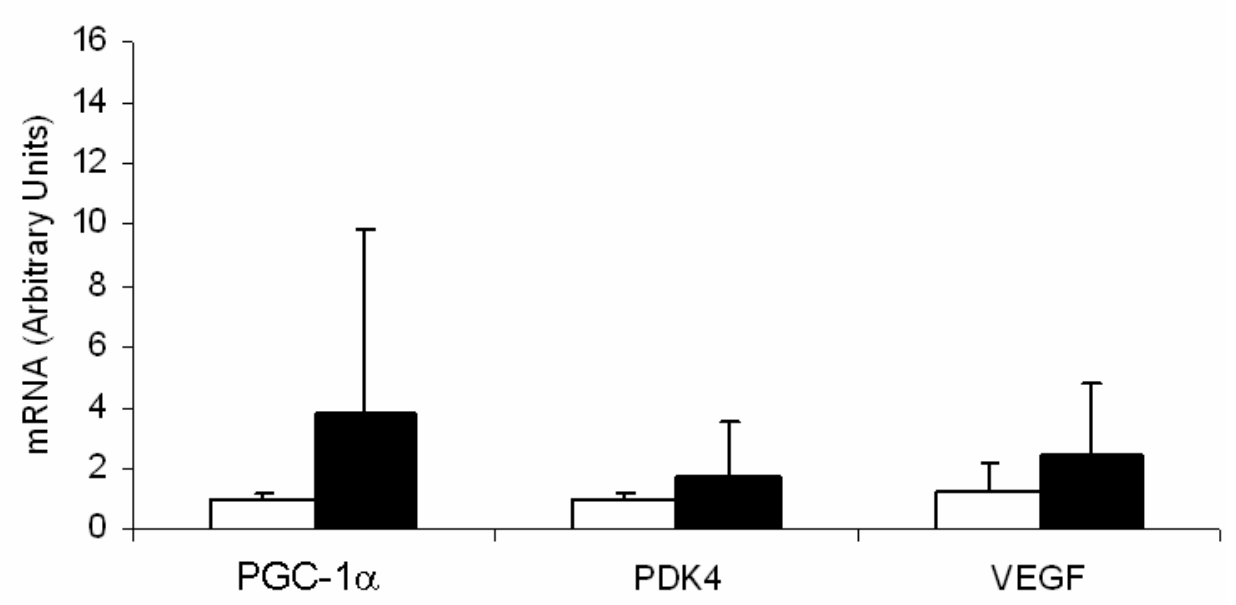

Figure 4.3 Changes in mRNA abundance for genes associated with endurance responses in (A) endurance-trained (ET; $n=6)$ and (B) strength-trained (ST; $n=7)$ subject vastus lateralis muscle sampled $3 \mathrm{~h}$ after $8 \times 5$ maximal effort leg extensions. All values are mean \pm SD. PGC$1 \alpha$, peroxisome proliferator activated receptor gamma co-activator $1 \alpha$; PDK4, pyruvate dehydrogenase kinase 4; VEGF, vascular endothelial growth factor. * Significant difference from rest (Repeated measures ANOVA, $\mathrm{P}<0.05$ ). 

A
ET Resistance
$\square$ Rest

a $\mathrm{h}$

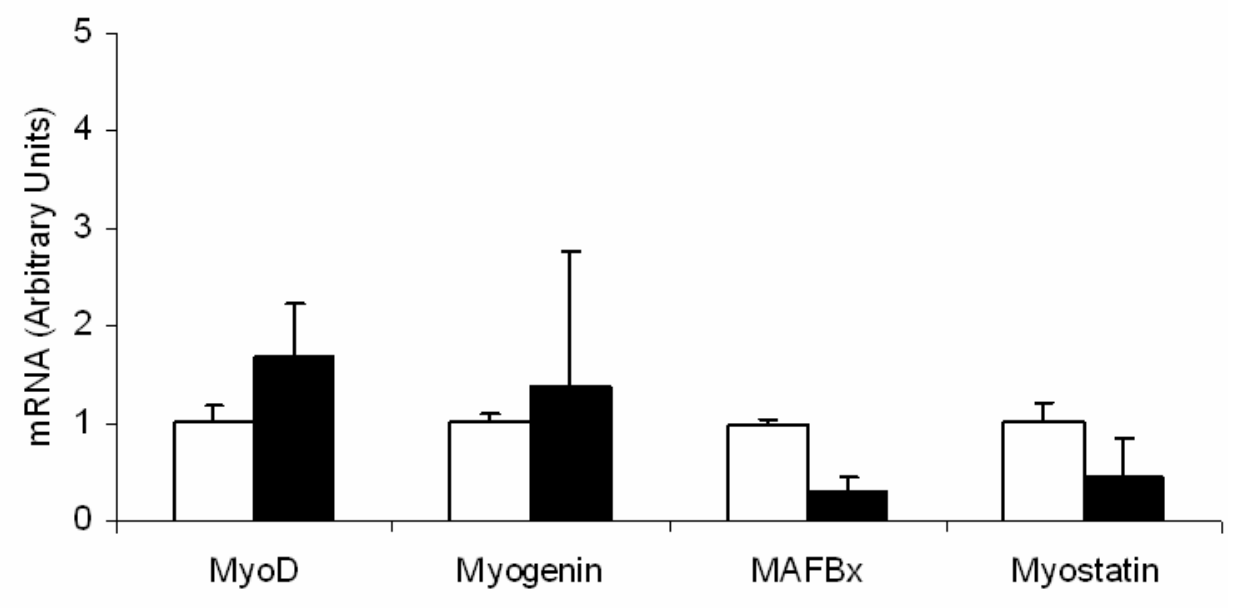

$\mathrm{B}$
ST Resistance
$\square$ Rest
B
STResistance
- $3 \mathrm{~h}$

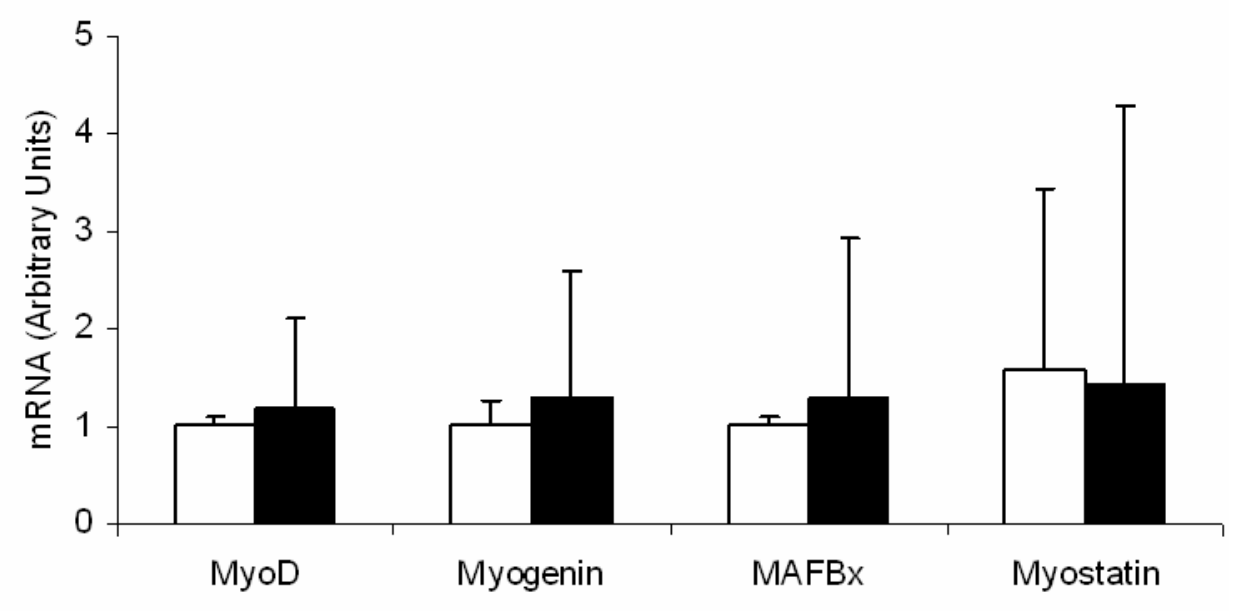

Figure 4.4 Changes in mRNA abundance for genes associated with myogenic responses in (A) endurance-trained (ET; $n=6)$ and (B) strength-trained $(\mathrm{ST} ; \mathrm{n}=7)$ subject vastus lateralis muscle sampled $3 \mathrm{~h}$ after $8 \times 5$ maximal effort leg extensions. All values are mean $\pm \mathrm{SD}$. MAFBx, muscle atrophy F-box protein. 


\subsection{Discussion}

Differences in muscle phenotype and subsequent function occur by activating and / or repressing different subsets of genes. A rate-limiting step for protein synthesis in response to exercise is the level of transcription and the quantity of mRNA abundance (Booth and Baldwin 1996; Sanders Williams and Neufer 1996), with the level of mRNA determined by the rates of mRNA synthesis and decay. New steady state protein synthesis via chronic and load-specific stimuli can optimise muscle tissue for such diverse physiological function as strength or endurance capabilities (Goldspink 2003; Grifone et al. 2004).

Previous studies have shown that diverse exercise stimuli induce specific early gene responses in skeletal muscle (Altschul et al. 1990; Pilegaard et al. 2000; Adams et al. 2004; Mahoney et al. 2005). However, the degree of specificity in gene responses to divergent modes of exercise when undertaken by athletes with an extensive history of single mode training has not been investigated. The methods of the present study employed exercise of the same relative rather than absolute exercise intensity in an attempt to mimic the habitual training practices of the two groups of athletes. For the first time, these data provide evidence demonstrating that divergent forms of exercise undertaken in habitually trained athletes results in the selective up-regulation / repression of specific gene sets that are likely to mediate some of the chronic exercise-induced adaptations to training.

The first novel finding of the present study was that a single bout of endurance exercise undertaken by both strength- and endurance-trained athletes elicited similar changes in the mRNA abundance of a subset of metabolic genes, namely PGC-1 $\alpha$, PDK4 and VEGF (Figure 4.1A, 1B). The results showing an up-regulation of these genes in endurance-trained subjects are in agreement with previous findings (Pilegaard et al. 2000; Gavin et al. 2004; Cartoni et al. 2005; Mahoney et al. 2005) and provide further support for their proposed roles in exercise-induced regulation of mitochondrial biogenesis, carbohydrate metabolism and angiogenesis, respectively (Pilegaard et al. 2000; Baar et al. 2002; Psilander et al. 2003; 
Gavin et al. 2004; LeBlanc et al. 2004). It has been proposed that adaptation to chronic resistance training does not create a favourable cellular environment with respect to oxidative potential (Tseng et al. 1994; Tseng et al. 1995; Hood 2001). The chronic training history of strength-trained athletes results in an increased cross-sectional area and fibre diameter of the trained musculature (MacDougall et al. 1982) and it has been suggested that this adaptation may limit nuclear and mitochondrial transcriptional capacity due to an increase in the cytoplasm to myonucleus ratio of the cell (Tseng et al. 1994). Furthermore, the mitochondrial density of enlarged fibres may be diluted, thus increasing diffusion distances for oxygen and substrates (Hood 2001). Notwithstanding these observations, such conditions did not preclude an endurance exercise-induced increase in "metabolic genes" in this subject population.

With regard to the acute mRNA responses of "myogenic genes" after a bout of cycling exercise, the present results demonstrate that MyoD and Myogenin increased in ET but not ST subjects (Figure 4.2A, 2B). MyoD and Myogenin are expressed in muscle satellite cells and mature myofibres and have been implicated in mediating the processes of cell proliferation and differentiation, and in defining muscle phenotype (Willoughby and Nelson 2002; Charge and Rudnicki 2004; Ishido et al. 2004b). Cycling involves minimal eccentric work for force production suggesting that the divergent responses of MyoD in the present study were not the result of disparity in the activation of satellite cell populations. The expression of $\mathrm{MyoD}$ is highly induced following resistance exercise, surgical ablation, denervation and muscle damage (Willoughby and Nelson 2002; Psilander et al. 2003; Ishido et al. 2004a; Ishido et al. 2004b; Bickel et al. 2005), but an increase in MyoD expression has not previously been reported after cycling exercise in humans. MyoD expression is increased after endurance treadmill running in rodents and humans (Armand et al. 2003; Yang et al. 2005). Yang and co workers (2005) observed increased MyoD mRNA abundance in physically active human subjects following 30 min of treadmill running, and attributed this to the eccentric component with foot strike and suggested that increased MyoD expression may 
indicate transcriptional regulation of fibre-specific alterations in myosin heavy chain expression. Previously, Kadi et al. (2004) have shown Myogenin accumulation following endurance cycling and this may represent an early signal mediating the control of myofibre oxidative metabolic properties. In support of this contention a significant increase in the mRNA abundance of myogenin following cycling exercise in ET subjects was observed. However, it is unclear why a decrease in Myogenin was observed in strength-trained subjects following cycling exercise.

If expression of myogenic regulatory factors occurs in mature myofibres as a result of endurance adaptation the decrease in MyoD and Myogenin mRNA in ST subjects following cycling may suggest a repressed response due to the adaptive phenotype of the muscle. However, although fibre-type specific responses are likely to contribute to the discrepancy in MyoD and Myogenin mRNA abundance following cycling exercise, the precise mechanism for such unique divergent responses in diversely trained athletic populations remains elusive.

A novel finding of the present study was the significant increase in mRNA abundance of several 'negative regulators' of muscle mass in both ET and ST subjects following cycling exercise. Increases in MAFBx and Myostatin after cycling exercise has not previously been reported (Figure 4.2A, 2B). MAFBx (also known as Atrogin-1) is an ubiquitin E3 ligase involved in the regulation of proteolysis (Lecker et al. 1999). The induction of MAFBx expression prior to muscle loss and its high expression during accelerated protein degradation strongly suggest that this plays an important role in initiating and maintaining proteolysis (Lecker et al. 1999; Gomes et al. 2001). Important regulators of MAFBx expression have been identified downstream of insulin and insulin-like growth factor signalling including AKT and the Forkhead box O (FoxO) class of transcription factors, and mammalian target of rapamycin (mTOR; Sandri et al. 2004; Glass 2005). Stimuli that have been shown to generate proteolysis in skeletal muscle include fasting and diabetes (Gomes et al. 2001; Dehoux et al. 2004). In the current study, it seems reasonable to conclude that the metabolic stress produced 
during 60 min endurance exercise performed in a fasted state contributed to the increase in MAFBx mRNA abundance. Moreover, the increase in MAFBx mRNA in both ET and ST subjects following cycling indicates a response that is independent of training history. Myostatin is a transforming growth factor defined as a negative regulator of muscle mass. Deletion of Myostatin is associated with gross muscle hypertrophy while over-expression results in lower muscle mass and decreased fibre size (McPherron and Lee 1997; ReiszPorszasz et al. 2003). In this study an increase in Myostatin mRNA after cycling in ET but not ST subjects was observed. These findings are in contrast to recent work in rodents after swimming exercise (Matsakas et al. 2005). Differences in muscle sampling time course and exercise mode and duration make comparisons difficult to rationalise these discordant findings. Previous work involving resistance exercise in humans has shown both decreased (Kim et al. 2005) and increased (Willoughby 2004) Myostatin mRNA expression. The specific mechanism through which Myostatin gene expression is controlled and how it exerts its effect on skeletal muscle is unclear. Nevertheless, the possibility exists that the skeletal muscle adaptive state in ST subjects as a result of chronic resistance training inhibits any acute up-regulation in Myostatin gene expression regardless of the exercise mode and that its regulation following endurance exercise occurs in a phenotype or genotype specific manner.

The patterns of induction of the two subsets of metabolic and myogenic genes after resistance exercise were variable. Considerable changes from rest were observed for PDK4, MyoD, MAFBx and Myostatin mRNA abundance in ET but not ST subjects following $3 \mathrm{~h}$ recovery after resistance exercise. PDK4 phosphorylates and inactivates the pyruvate dehydrogenase complex and facilitates movement of glycolytically derived pyruvate toward lactate output rather than oxidation (Sugden and Holness 2003). The increase in PDK4 in ET subjects following resistance exercise probably corresponds to a requirement for increased glycolytic metabolism to supply energy to sustain the repeated high-intensity contractile activity, and may also have enhanced muscle glycogen resynthesis during recovery. The 
present data are in agreement with those of Yang et al. (2005) showing increased PDK4 mRNA expression following resistance exercise.

Compatible with its role in muscle hypertrophy and supporting the work of others (Willoughby and Nelson 2002; Psilander et al. 2003; Bickel et al. 2005), MyoD mRNA increased in ET subjects following resistance exercise. The role of MyoD in satellite cell proliferation and differentiation for subsequent muscle regeneration and hypertrophy has been previously noted. Resistance training induces muscle overload resulting in local myotrauma that stimulates a number of processes including satellite cell activation (Hawke and Garry 2001). Therefore, unfamiliar resistance exercise in ET subjects might have been expected to induce an increase in MyoD expression due to the activation of satellite cells for muscle repair / regeneration and as an adaptation response for greater force production and reduced cellular disturbance with subsequent repeated exercise bouts. MAFBx is a mediator of atrophy involved in the breakdown of muscle protein while Myostatin negatively regulates hypertrophy processes (Langley et al. 2002; Hoffman and Nader 2004). Few studies have investigated the effect of exercise on MAFBx and Myostatin expression in humans and the specific response to resistance exercise is still to be determined. Nevertheless, preliminary evidence implicates resistance exercise in the down-regulation of the mRNA content of both MAFBx and Myostatin, almost certainly reducing their negative affects on subsequent hypertrophy processes (Roth et al. 2003; Jones et al. 2004; Kim et al. 2005). The results from this study provide evidence in support of such a contention.

In conclusion, an important component of this work was the elucidation of whether familiar / unfamiliar exercise induced a suppressed or amplified acute response in subsets of 'metabolic' and 'myogenic' genes that are likely to be fundamental to specific exerciseinduced adaptation. A single endurance exercise bout produced comparative transcriptional responses in metabolic genes while training history markedly altered the response of myogenic genes. Few differences were evident following resistance exercise, however the 
magnitude of exercise response was notably larger in endurance-trained subjects. A potential limitation with regard to the interpretation of the current data set is the use of a single postexercise time point to evaluate the responses of the exercise-induced genes under investigation. Muscle samples were collected $3 \mathrm{~h}$ after the completion of an exercise bout because previous studies have reported that for the genes of interest determined in the present investigation, transcriptional activation occurs primarily during the initial few hours of recovery (Pilegaard et al. 2000; Psilander et al. 2003; Gavin et al. 2004; Matsakas et al. 2005; Yang et al. 2005). The results from the present study indicate that independent of exercise mode, the gene responses in skeletal muscle from endurance-trained athletes appears to be more sensitive to alterations in the cellular milieu than strength-trained subjects. This may indicate that a greater degree of muscle plasticity is conserved with regard to the acute mRNA responses compared to their strength-trained counterparts. Alternatively, the chronic adaptive state of muscle from strength-trained athletes may require a greater overload stimulus or repeated bouts of exercise to increase the transcriptional activity of myogenic genes in response to resistance exercise. 


\section{Chapter Five}

Summary and Conclusions 
The principle aims of the studies undertaken for this thesis were to enhance our current understanding of the molecular responses in skeletal muscle following resistance and endurance training overload. Both rodent and human in vivo exercise models were employed to elucidate the effect of overload frequency, exercise mode and muscle phenotype on these responses. The aims of this thesis were to 1) systematically evaluate the time-course of a spectrum of cellular and molecular markers specific to the regulation of muscle contractile protein and subsequent hypertrophy, after the imposition of different resistance training protocols and 2) to quantify the early response to divergent training stimuli in competitive athletes who are highly specialised / adapted to a single discipline, and to establish the degree of "signalling specificity" in these athletes.

The first study (Chapter 2) used a high-frequency model of training and evaluated the subsequent time-course of signalling responses. Specifically, this study compared conventional-frequency resistance training to a novel high-frequency "stacked" resistance training regimen and examined the response of kinases implicated in hypertrophy, inflammation and atrophy signalling pathways. Activation of intra-cellular signal transduction in skeletal muscle following contractile activity is a transient response that promotes or inhibits subsequent gene expression and adaptation. Consequently, the hypothesis of this investigation was that if a subsequent exercise bout was performed while the anabolic signalling response to an initial bout was still elevated, this could potentially extend the duration and / or amplitude of the signal, "stacking" the adaptive response onto that initiated previously. Contrary to the original hypothesis that "stacked" training would amplify and extend the anabolic signalling response, the first novel finding from this study was an extended inflammatory / atrophy signalling response after high-frequency work bouts. Moreover, high-frequency work bouts induced a co-ordinated and sustained elevation of inflammatory cytokine signalling. The results from this study suggest that in previously 
untrained muscle high-frequency resistance training does not enhance the activation of anabolic pathways and exacerbates inflammation and atrophy signal transduction.

Study one also provides new information on the effect of conventional resistance training on inflammatory responses in skeletal muscle. In contrast to high-frequency resistance training, after the initial bout of exercise conventional-frequency resistance training induced a suppression of inflammatory signalling responses following subsequent exercise bouts. Moreover, the signalling responses of inflammatory cytokines following three training bouts were not significantly different to control values and after a further $48 \mathrm{~h}$ recovery decreased below these levels. Thus, the capacity for exercise to induce a pro- or antiinflammatory effect in a frequency-dependent manner highlights the importance of recovery in determining subsequent adaptive events and in promoting or inhibiting the specificity of training adaptation. In addition, these results indicate a rapid reduction in the inflammatory response with repeated bouts of exercise in previously untrained muscle. Such information has important implications for disorders characterised by chronic skeletal muscle inflammation (e.g. type 2 diabetes). Indeed, the reduction in TNF $\alpha$ signalling observed with conventional training provides support for the capacity of habitual exercise to reduce the activity of inflammatory pathways in skeletal muscle.

The second study (Chapter 3 and 4) compared the signalling and mRNA response of highly-trained strength and endurance athletes to habitual and unfamiliar exercise. Specifically, strength and endurance athletes undertook a bout of resistance or cycling exercise, respectively, then "crossed-over" and performed a bout of exercise they do not normally incorporate into their habitual training regimen. The distinct molecular events that characterise adaptation to divergent exercise such as resistance and endurance training appear to be incompatible. Therefore, the hypothesis was that when athletes undertook a training bout in an unfamiliar exercise discipline, the adaptive phenotype of the muscle would abolish the expected molecular response associated with the specificity of training. In contrast to this 
hypothesis, the results from this study indicate "cross-over" to an unfamiliar exercise mode does not prevent a training-specific adaptive response. However, prior training history had a profound effect on signalling and mRNA responses to the habitual training modes.

AMPK has been implicated in an "aerobic" adaptation pathway resulting in increased fat utilisation and mitochondrial biogenesis, while AKT has been identified as an important regulator of hypertrophy and cell growth in skeletal muscle. The results from this study provide new information demonstrating that when endurance athletes performed cycling there was little activation of the AMPK pathway. Likewise, when strength-trained athletes undertook resistance exercise there was little up-regulation of the AKT signalling pathways. Conversely, when athletes "crossed-over" and performed a bout of unfamiliar exercise, strength- and endurance-trained subjects significantly increased AMPK activation. The results from this study also demonstrated that strength-trained athletes did not promote / inhibit mRNA abundance of genes regulating muscle mass after resistance training but that cycling induced a significant increase in the mRNA abundance of genes associated with aerobic metabolism. Thus, it appears the adaptive phenotype resulting from chronic resistance training retains a capacity to increase metabolic gene expression in response to endurance training. Collectively, these findings have a number of important ramifications regarding our current understanding of the specificity of exercise-induced adaptation responses.

Foremost, it appears that a single bout of habitual exercise in a highly adapted phenotype is insufficient to induce significant increases in the molecular responses associated with the desired exercise-specific adaptation. This highlights the importance of overload in initiating mechanotransduction and subsequent primary and secondary messenger activation to promote adaptation in elite athletes. Also, the increase in PGC-1 $\alpha$ mRNA abundance despite the lack of AMPK activation when endurance athletes undertook cycling indicates that AMPK activity may not exclusively promote mitochondrial biogenesis but is a consequence of intense contraction-induced overload. These findings raise the question of the putative role 
of AMPK in the adaptation to endurance training. In addition, this study provides novel data to show that prolonged cycling increases mRNA abundance of genes implicated in the negative regulation of muscle mass. This suggests that endurance training may up-regulate proteolysis and suppress hypertrophy thereby impairing the muscles capacity to increase protein synthesis and muscle mass. Indeed, Akt has been implicated in down-regulating ubiquitin-ligase atrophy processes by inhibiting the FoxO-MAFBx pathway. The lack of Akt phosphorylation after cycling exercise was performed by strength-trained athletes likely promoted the transcriptional activity of atrophy genes. Equally, the AMPK-mediated inhibition of mTOR signalling and subsequent reductions in translational efficiency following aerobic exercise may have exacerbated protein degradation and contributed to increased MAFBx and Myostatin mRNA abundance in the strength athletes. Collectively, these data provide potential mechanisms for adaptation interference, suggesting that the dominant mechanism of interference with concurrent training is the negative effect of aerobic metabolism on pathways regulating protein synthesis and degradation.

In conclusion, the aim of training is to provide an overload stimulus that generates specific molecular responses to enhance the adaptive phenotype. In this regard, key regulators of skeletal muscle adaptation are emerging that are likely to significantly contribute to promoting the specificity of training responses, driving the muscle phenotype to either ends of an adaptation continuum. Thus, endurance training should activate pathways to promote adaptation toward enhanced oxidative capacity and resistance to fatigue during prolonged contractile activity. Conversely, resistance training should up-regulate translational machinery and satellite cell activity increasing protein synthesis and muscle cross-sectional area.

The results from the studies undertaken for this thesis provide novel information regarding the effect of overload frequency, exercise mode and training history on the molecular bases of training adaptation. However, several important questions remain that should be addressed in future work. For example, a high-frequency training stimulus suppresses 
the adaptive response in untrained subjects (Chapter 2), yet a single training bout of habitual exercise undertaken by highly-trained athletes was insufficient to generate further adaptation responses (Chapter 3). It is unclear how periods of high-frequency training might affect the molecular adaptation response in trained athletes. Furthermore, the unique signalling events that occur when trained individuals undertake exercise in an "unfamiliar" activity (i.e. "interference") is not well understood (Chapter 4). If "crossing over" to an alternate exercise mode interferes with adaptive responses initiated during prior training sessions (i.e. concurrent training), this is likely to suppress or limit the specific exercise response and produce a less than optimal adaptation. Moreover, the effect of alternating endurance and resistance training sessions on the strength-endurance adaptation continuum remains elusive.

The adaptation continuum provides a framework with which to assess the molecular bases of training adaptation. However, this simplistic approach characterising the adaptation to endurance and resistance training does not address the multifaceted nature of training specificity. This is undoubtedly complicated by the addition of other training modes, nutritional interventions and recovery modalities. Nonetheless, continued discovery of mechanisms involved in regulating the adaptation response will enhance our understanding of the specificity of training adaptation. Greater knowledge regarding exercise-induced adaptation in skeletal muscle requires the application of innovative training interventions to promote and extend our current understanding of adaptive events that may ultimately translate to novel training practices for athletic endeavour. Understanding the specificity of training adaptation is not only important for sport and exercise scientists but may also provide therapeutic targets for the treatment of acute and chronic skeletal muscle diseases. 


\title{
Chapter Eight
}

\author{
References
}


Abu Hatoum, O., Gross-Mesilaty, S., Breitschopf, K., Hoffman, A., Gonen, H., Ciechanover, A., and Bengal, E. 1998. Degradation of Myogenic Transcription Factor MyoD by the Ubiquitin Pathway In Vivo and In Vitro: Regulation by Specific DNA Binding. Mol. Cell. Biol. 18: 5670-5677.

Adams, G.R., Cheng, D.C., Haddad, F., and Baldwin, K.M. 2004. Skeletal muscle hypertrophy in response to isometric, lengthening, and shortening training bouts of equivalent duration. J Appl Physiol 96: 1613-1618.

Adams, G.R., Haddad, F., and Baldwin, K.M. 1999. Time course of changes in markers of myogenesis in overloaded rat skeletal muscles. J Appl Physiol 87: 1705-1712.

Adams, G.R., and McCue, S.A. 1998. Localized infusion of IGF-I results in skeletal muscle hypertrophy in rats. $J$ Appl Physiol 84: 1716-1722.

Adhihetty, P.J., Irrcher, I., Joseph, A.M., Ljubicic, V., and Hood, D.A. 2003. Plasticity of skeletal muscle mitochondria in response to contractile activity. Exp Physiol 88: 99107.

Ahtiainen, J.P., Pakarinen, A., Alen, M., Kraemer, W.J., and Häkkinen, K. 2003. Muscle hypertrophy, hormonal adaptations and strength development during strength training in strength-trained and untrained men. Eur J Appl Physiol 89: 555-563.

Ai, H., Ihlemann, J., Hellsten, Y., Lauritzen, H.P.M.M., Hardie, D.G., Galbo, H., and Ploug, T. 2002. Effect of fiber type and nutritional state on AICAR- and contractionstimulated glucose transport in rat muscle 10.1152/ajpendo.00167.2001. Am J Physiol Endocrinol Metab 282: E1291-1300.

Akimoto, T., Pohnert, S.C., Li, P., Zhang, M., Gumbs, C., Rosenberg, P.B., Williams, R.S., and Yan, Z. 2005. Exercise Stimulates PGC-1 $\alpha$ Transcription in Skeletal Muscle through Activation of the p38 MAPK Pathway. J. Biol. Chem. 280: 19587-19593.

Akimoto, T., Ribar, T.J., Williams, R.S., and Yan, Z. 2004. Skeletal muscle adaptation in response to voluntary running in $\mathrm{Ca} 2+/$ calmodulin-dependent protein kinase IVdeficient mice 10.1152/ajpcell.00248.2004. Am J Physiol Cell Physiol 287: C13111319.

Alenghat, F.J., and Ingber, D.E. 2002. Mechanotransduction: All Signals Point to Cytoskeleton, Matrix, and Integrins 10.1126/stke.2002.119.pe6. Sci. STKE 2002: pe6-.

Alessi, D.R., James, S.R., Downes, C.P., Holmes, A.B., Gaffney, P.R.J., Reese, C.B., and Cohen, P. 1997. Characterization of a 3-phosphoinositide-dependent protein kinase which phosphorylates and activates protein kinase B $\alpha$. Current Biology 7: 261-269.

Alessi, D.R., Kozlowski, M.T., Weng, Q.-P., Morrice, N., and Avruch, J. 1998. 3Phosphoinositide-dependent protein kinase 1 (PDK1) phosphorylates and activates the p70 S6 kinase in vivo and in vitro. Current Biology 8: 69-81.

Ali, S.M., and Sabatini, D.M. 2005. Structure of S6 Kinase 1 Determines whether RaptormTOR or Rictor-mTOR Phosphorylates Its Hydrophobic Motif Site. J. Biol. Chem. 280: $19445-19448$.

Alkalay, I., Yaron, A., Hatzubai, A., Orian, A., Ciechanover, A., and Ben-Neriah, Y. 1995.

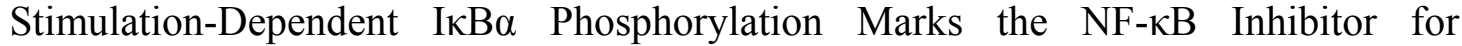
Degradation Via the Ubiquitin-Proteasome Pathway 10.1073/pnas.92.23.10599. PNAS 92: 10599-10603.

Allen, T., Byrd, R., and Smith, D. 1976. Hemodynamic consequences of circuit weight training. Res $Q$ 47: 229-306.

Altschul, S.F., Gish, W., Miller, W., Meyers, E.W., and Lipman, D.J. 1990. Basic Local Alignment Search Tool. J Mol Biol 215: 403-410.

Arbogast, S., and Reid, M.B. 2004. Oxidant activity in skeletal muscle fibers is influenced by temperature, CO2 level, and muscle-derived nitric oxide 10.1152/ajpregu.00072.2004. Am J Physiol Regul Integr Comp Physiol 287: R698-705. 
Armand, A.-S., Launay, T., Gaspera, B.D., Charbonnier, F., Gallien, C.L., and Chanoine, C. 2003. Effects of eccentric treadmill running on mouse soleus: degeneration/regeneration studied with Myf-5 and MyoD probes. Acta Physiol Scand 179: $75-84$.

Aronson, D., Violan, M.A., Dufresne, S.D., Zangen, D., Fielding, R.A., and Goodyear, L.J. 1997. Exercise Stimulates the Mitogen-activated Protein Kinase Pathway in Human Skeletal Muscle. J. Clin. Invest. 99: 1251-1257.

Aschenbach, W.G., Sakamoto, K., and Goodyear, L.J. 2004. 5' Adenosine monophosphateactivated protein kinase, metabolism and exercise. Sports Med 34: 91-103.

Assoian, R.K. 2002. Common sense signalling. Nat Cell Biol 4: E187-E188.

Atherton, P.J., Babraj, J.A., Smith, K., Singh, J., Rennie, M.J., and Wackerhage, H. 2005. Selective activation of AMPK-PGC-1 $\alpha$ or PKB-TSC2-mTOR signaling can explain specific adaptive responses to endurance or resistance training-like electrical muscle stimulation. FASEB J.: 04-2179fje.

Baar, K., Blough, E., Dineen, B., and Esser, K. 1999. Transcriptional regulation in response to exercise. Exerc Sport Sci Rev 27: 333-379.

Baar, K., and Esser, K. 1999. Phosphorylation of $\mathrm{p} 70^{\mathrm{S} 6 \mathrm{k}}$ correlates with increased skeletal muscle mass following resistance exercise. Am J Physiol Cell Physiol 276: C120C127.

Baar, K., Wende, A.R., Jones, T.E., Marison, M., Nolte, L.A., Chen, M., Kelly, D.P., and Holloszy, J.O. 2002. Adaptations of skeletal muscle to exercise: rapid increase in the transcriptional coactivator PGC-1. FASEB J. 16: 1879-1886.

Bach, D., Pich, S., Soriano, F.X., Vega, N., Baumgartner, B., Oriola, J., Daugaard, J.R., Lloberas, J., Camps, M., Zierath, J.R., et al. 2003. Mitofusin-2 determines mitochondrial network architecture and mitochondrial metabolism. J Biol Chem 278: 17190-17197.

Backer, J., Myers, M.J., Shoelson, S., Chin, D., Sun, X., Miralpeix, M., Hu, P., Margolis, B., Skolnik, E., and Schlessinger, J.e.a. 1992. Phosphatidylinositol 3'-kinase is activated by association with IRS-1 during insulin stimulation. EMBO J 11: 3469-3479.

Bae, J.S., Jang, M.K., Hong, S., An, W.G., Choi, Y.H., Kim, H.D., and Cheong, J. 2003. Phosphorylation of NF- $\kappa$ B by calmodulin-dependent kinase IV activates anti-apoptotic gene expression. Biochem Biophys Res Commun 305: 1094-1098.

Balabinis, C.P., Moukas, M., Behrakis, P.K., Psarakis, C.H., and Vassiliou, M.P. 2003. Early phase changes by concurrent endurance and strength training. J Strength Cond Res 17: 393-401.

Bamman, M.M., Shipp, J.R., Jiang, J., Gower, B.A., Hunter, G.R., Goodman, A., McLafferty, C.L., Jr., and Urban, R.J. 2001. Mechanical load increases muscle IGF-I and androgen receptor mRNA concentrations in humans. Am J Physiol Endocrinol Metab 280: E383-390.

Bangsbo, J., Krustrup, P., Gonzalez-Alonso, J., and Saltin, B. 2001. ATP production and efficiency of human skeletal muscle during intense exercise: effect of previous exercise. Am J Physiol Endocrinol Metab 280: E956-964.

Bassel-Duby, R., and Olson, E.N. 2006. Signaling pathways in skeletal muscle remodeling. Annu Rev Biochem 75: 19-37.

Bell, G.J., Syrotuik, D.G., Martin, T.P., Burnham, R., and Quinney, H.A. 2000. Effect of concurrent strength and endurance training on skeletal muscle properties and hormone concentrations in humans. Eur J Appl Physiol 81: 481-427.

Bengtsson, J., Gustafsson, T., Widegren, U., Jansson, E., and Sundberg, C.J. 2001. Mitochondrial transcription factor A and respiratory complex IV increase in response to exercise training in humans. Pflugers Arch 443: 61-66. 
Bickel, C.S., Slade, J., Mahoney, E., Haddad, F., Dudley, G.A., and Adams, G.R. 2005. Time course of molecular responses of human skeletal muscle to acute bouts of resistance exercise. J Appl Physiol 98: 482-488.

Bickel, C.S., Slade, J.M., Haddad, F., Adams, G.R., and Dudley, G.A. 2003. Acute molecular responses of skeletal muscle to resistance exercise in able-bodied and spinal cordinjured subjects. J Appl Physiol 94: 2255-2262.

Bodine, S.C., Latres, E., Baumhueter, S., Lai, V.K.-M., Nunez, L., Clarke, B.A., Poueymirou, W.T., Panaro, F.J., Na, E., Dharmarajan, K., et al. 2001a. Identification of Ubiquitin Ligases Required for Skeletal Muscle Atrophy. Science 294: 1704-1708.

Bodine, S.C., Stitt, T.N., Gonzalez, M., Kline, W.O., Stover, G.L., Bauerlein, R., Zlotchenko, E., Scrimgeour, A., Lawerence, J.C., Glass, D.J., et al. 2001b. Akt/mTOR pathway is a crucial regulator of skeletal muscle hypertrophy and can prevent muscle atrophy in vivo. Nat Cell Biol. 3: 1014-1019.

Bolster, D.R., Crozier, S.J., Kimball, S.R., and Jefferson, L.S. 2002. AMP-activated Protein Kinase Suppresses Protein Synthesis in Rat Skeletal Muscle through Down-regulated Mammalian Target of Rapamycin (mTOR) Signaling 10.1074/jbc.C200171200. J. Biol. Chem. 277: 23977-23980.

Bolster, D.R., Kimball, S.R., and Jefferson, L.S. 2003a. Translational control mechanisms modulate skeletal muscle gene expression during hypertrophy. Exerc Sport Sci Rev 31: 111-116.

Bolster, D.R., Kubica, N., Crozier, S.J., Williamson, D.L., Farrell, P.A., Kimball, S.R., and Jefferson, L.S. 2003b. Immediate response of mammalian target of rapamycin (mTOR)-mediated signalling following acute resistance exercise in rat skeletal muscle. J Physiol (Lond) 553: 213-220.

Booth, F.W., and Baldwin, K.M. 1996. Muscle Plasticity: energy demand and supply processes. In Handbook of Physiology. Section 12: Exercise: Regulation and Intergration of Multiple Systems. (eds. L.B. Rowell, and J.T. Shepherd), pp. 10751123. Oxford University Press, New York.

Booth, F.W., and Thomason, D.B. 1991. Molecular and cellular adaptation of muscle in response to exercise: perspectives of various models. Physiol. Rev. 71: 541-585.

Booth, F.W., and Watson, P.A. 1985. Control of adaptations in protein levels in response to exercise. Fed Proc 44: 2293-2300.

Boppart, M.D., Asp, S., Wojtaszewski, J.F.P., Fielding, R.A., Mohr, T., and Goodyear, L.J. 2000. Marathon running transiently increases c-Jun NH2-terminal kinase and p38 $\gamma$ activities in human skeletal muscle. $J$ Physiol (Lond) 526: 663-669.

Bosisio, D., Marazzi, I., Agresti, A., Shimizu, N., Bianchi, M., and Natoli, G. 2006. A hyperdynamic equilibrium between promoter-bound and nucleoplasmic dimers controls NF$\kappa \mathrm{B}$-dependent gene activity. EMBO J 25: 789-810.

Bouchard, C., Malina, R., and Pérusse, L. 1997. On the Horizon: Molecular Biology - a new vista for exercise physiology. In Genetics of Fitness and Physical Performance, pp. 970-1050. Human Kinetics, Champaign, Il.

Bouchard, C., Rankinen, T., Chagnon, Y.C., Rice, T., Perusse, L., Gagnon, J., Borecki, I., An, P., Leon, A.S., Skinner, J.S., et al. 2000. Genomic scan for maximal oxygen uptake and its response to training in the HERITAGE Family Study. J Appl Physiol 88: 551559.

Browne, G.J., and Proud, C.G. 2002. Regulation of peptide-chain elongation in mammalian cells. Eur J Biochem 269: 5360-5368.

Browne, G.J., and Proud, C.G. 2004. A Novel mTOR-Regulated Phosphorylation Site in Elongation Factor 2 Kinase Modulates the Activity of the Kinase and Its Binding to Calmodulin. Mol. Cell. Biol. 24: 2986-2997.

Brozinick Jr., J.T., and Birnbaum, M.J. 1998. Insulin, but Not Contraction, Activates Akt/PKB in Isolated Rat Skeletal Muscle. J. Biol. Chem. 273: 14679-14682. 
Bruss, M.D., Arias, E.B., Lienhard, G.E., and Cartee, G.D. 2005. Increased Phosphorylation of Akt Substrate of $160 \mathrm{kDa}$ (AS160) in Rat Skeletal Muscle in Response to Insulin or Contractile Activity. Diabetes 54: 41-50.

Burnett, P.E., Barrow, R.K., Cohen, N.A., Snyder, S.H., and Sabatini, D.M. 1998. RAFT1 phosphorylation of the translational regulators p70 S6 kinase and 4E-BP1. PNAS 95: 1432-1437.

Byrd, S.K., McCutcheon, L.J., Hodgson, D.R., and Gollnick, P.D. 1989. Altered sarcoplasmic reticulum function after high-intensity exercise. J Appl Physiol 67: 2072-2077.

Cai, D., Frantz, J.D., Tawa, J., Nicholas E., Melendez, P.A., Oh, B.-C., Lidov, H.G.W., Hasselgren, P.-O., Frontera, W.R., and Lee, J. 2004. IKK $\beta / N F-\kappa B$ Activation Causes Severe Muscle Wasting in Mice. Cell 119: 285-298.

Cai, S.-L., Tee, A.R., Short, J.D., Bergeron, J.M., Kim, J., Shen, J., Guo, R., Johnson, C.L., Kiguchi, K., and Walker, C.L. 2006. Activity of TSC2 is inhibited by AKT-mediated phosphorylation and membrane partitioning 10.1083/jcb.200507119. J. Cell Biol. 173: 279-289.

Cannon, J.G., and St. Pierre, B.A. 1998. Cytokines in exertion-induced skeletal muscle injury. Mol Cell Biochem 179: 159-167.

Carling, D., Zammit, V.A., and Hardie, D.G. 1987. A common bicyclic protein kinase cascade inactivates the regulatory enzymes of fatty acid and cholesterol biosynthesis. FEBS Letters 223: 217-222.

Carrero, P., Okamoto, K., Coumailleau, P., O'Brien, S., Tanaka, H., and Poellinger. 2000. Redox-regulated recruitment of the transcriptional coactivators CREB-binding protein and SRC-1 to hypoxia-inducible factor alpha. Mol Cell Biol 20: 402-415.

Carroll, S.L., Klein, M.G., and Schneider, M.F. 1995. Calcium transients in intact rat skeletal muscle fibers in agarose gel. Am J Physiol Cell Physiol 269: C28-34.

Cartoni, R., Leger, B., Hock, M.B., Praz, M., Crettenand, A., Pich, S., Ziltener, J.-L., Luthi, F., Deriaz, O., Zorzano, A., et al. 2005. Mitofusins 1/2 and ERR $\alpha$ expression are increased in human skeletal muscle after physical exercise. J Physiol 567: 349-358.

Chakravarthy, M.V., Abraha, T.W., Schwartz, R.J., Fiorotto, M.L., and Booth, F.W. 2000a. Insulin-like Growth Factor-I Extends in Vitro Replicative Life Span of Skeletal Muscle Satellite Cells by Enhancing G1/S Cell Cycle Progression via the Activation of Phosphatidylinositol 3'-Kinase/Akt Signaling Pathway. J. Biol. Chem. 275: 3594235952.

Chakravarthy, M.V., Davis, B.S., and Booth, F.W. 2000b. IGF-I restores satellite cell proliferative potential in immobilized old skeletal muscle. J Appl Physiol 89: 13651379.

Charge, S.B.P., and Rudnicki, M.A. 2004. Cellular and Molecular Regulation of Muscle Regeneration. Physiol. Rev. 84: 209-238.

Chen, Z., Hagler, J., Palombella, V., Melandri, F., Scherer, D., Ballard, D., and Maniatis, T. 1995. Signal-induced site-specific phosphorylation targets $\mathrm{I} \kappa \mathrm{B} \alpha$ to the ubiquitinproteasome pathway. Genes Dev 9: 1586-1597.

Chen, Z.J. 2005. Ubiquitin signalling in the NF-[kappa]B pathway. 7: 758-765.

Chen, Z.J., Bhoj, V., and Seth, R.B. 2006. Ubiquitin, TAK1 and IKK: is there a connection? Cell Death Differ 13: 687-692.

Chen, Z.-P., McConell, G.K., Michell, B.J., Snow, R.J., Canny, B.J., and Kemp, B.E. 2000. AMPK signaling in contracting human skeletal muscle: acetyl-CoA carboxylase and NO synthase phosphorylation. Am J Physiol Endocrinol Metab 279: E1202-1206.

Chen, Z.-P., Stephens, T.J., Murthy, S., Canny, B.J., Hargreaves, M., Witters, L.A., Kemp, B.E., and McConell, G.K. 2003. Effect of Exercise Intensity on Skeletal Muscle AMPK Signaling in Humans. Diabetes 52: 2205-2212. 
Chesley, A., MacDougall, J.D., Tarnopolsky, M.A., Atkinson, S.A., and Smith, K. 1992. Changes in human muscle protein synthesis after resistance exercise. $J$ Appl Physiol 73: $1383-1388$.

Chin, E.R. 2005. Role of Ca2+/calmodulin-dependent kinases in skeletal muscle plasticity 10.1152/japplphysiol.00015.2005. J Appl Physiol 99: 414-423.

Chin, E.R., Olson, E.N., Richardson, J.A., Yang, Q., Humphries, C., Shelton, J.M., Wu, H., Zhu, W., Bassel-Duby, R., and Williams, R.S. 1998. A calcineurin-dependent transcriptional pathway controls skeletal muscle fiber type 10.1101/gad.12.16.2499. Genes Dev. 12: 2499-2509.

Clark, S.A., Chen, Z.-P., Murphy, K.T., Aughey, R.J., McKenna, M.J., Kemp, B.E., and Hawley, J.A. 2004. Intensified exercise training does not alter AMPK signaling in human skeletal muscle. Am J Physiol Endocrinol Metab 286: E737-743.

Cluberton, L.J., McGee, S.L., Murphy, R.M., and Hargreaves, M. 2005. Effect of carbohydrate ingestion on exercise-induced alterations in metabolic gene expression. $J$ Appl Physiol 99: 1359-1363.

Connor, M.K., Irrcher, I., and Hood, D.A. 2001. Contractile Activity-induced Transcriptional Activation of Cytochrome c Involves Sp1 and Is Proportional to Mitochondrial ATP Synthesis in C2C12 Muscle Cells. J. Biol. Chem. 276: 15898-15904.

Cornelison, D.D.W., Olwin, B.B., Rudnicki, M.A., and Wold, B.J. 2000. MyoD-/- Satellite Cells in Single-Fiber Culture Are Differentiation Defective and MRF4 Deficient. Dev Biol 224: 122-137.

Cornelison, D.D.W., and Wold, B.J. 1997. Single-Cell Analysis of Regulatory Gene Expression in Quiescent and Activated Mouse Skeletal Muscle Satellite Cells. Dev Biol 191: 270-283.

Creer, A., Gallagher, P., Slivka, D., Jemiolo, B., Fink, W., and Trappe, S. 2005. Influence of muscle glycogen availability on ERK1/2 and Akt signaling after resistance exercise in human skeletal muscle. J Appl Physiol 99: 950-956.

Cross, D.A., Alessi, D.R., Cohen, P., Andjelkovich, M., and Hemmings, B.A. 1995. Inhibition of glycogen synthase kinase-3 by insulin mediated by protein kinase B. Nature. 378: 785-789.

Cuthbertson, D., Smith, K., Babraj, J., Leese, G., Waddell, T., Atherton, P., Wackerhage, H., Taylor, P.M., and Rennie, M.J. 2005. Anabolic signaling deficits underlie amino acid resistance of wasting, aging muscle. FASEB J. 19: 422-424.

Czerwinski, S.M., Martin, J.M., and Bechtel, P.J. 1994. Modulation of IGF mRNA abundance during stretch-induced skeletal muscle hypertrophy and regression. J Appl Physiol 76: 2026-2030.

Daitoku, H., Yamagata, K., Matsuzaki, H., Hatta, M., and Fukamizu, A. 2003. Regulation of PGC-1 Promoter Activity by Protein Kinase B and the Forkhead Transcription Factor FKHR. Diabetes 52: 642-649.

de Alvaro, C., Teruel, T., Hernandez, R., and Lorenzo, M. 2004. Tumor Necrosis Factor $\alpha$ Produces Insulin Resistance in Skeletal Muscle by Activation of Inhibitor $\kappa B$ Kinase in a p38 MAPK-dependent Manner. J. Biol. Chem. 279: 17070-17078.

Dehoux, M., Van Beneden, R., Pasko, N., Lause, P., Verniers, J., Underwood, L., Ketelslegers, J.-M., and Thissen, J.-P. 2004. Role of the Insulin-Like Growth Factor I Decline in the Induction of Atrogin-1/MAFbx during Fasting and Diabetes. Endocrinology 145: 4806-4812.

del Aguila, L.F., Claffey, K.P., and Kirwan, J.P. 1999. TNF- $\alpha$ impairs insulin signaling and insulin stimulation of glucose uptake in $\mathrm{C} 2 \mathrm{C} 12$ muscle cells. Am J Physiol Endocrinol Metab 276: E849-855.

Del Aguila, L.F., Krishnan, R.K., Ulbrecht, J.S., Farrell, P.A., Correll, P.H., Lang, C.H., Zierath, J.R., and Kirwan, J.P. 2000. Muscle damage impairs insulin stimulation of 
IRS-1, PI 3-kinase, and Akt-kinase in human skeletal muscle. Am J Physiol Endocrinol Metab 279: E206-212.

DeVol, D.L., Rotwein, P., Sadow, J.L., Novakofski, J., and Bechtel, P.J. 1990. Activation of insulin-like growth factor gene expression during work-induced skeletal muscle growth. Am J Physiol Endocrinol Metab 259: E89-95.

Dreyer, H.C., Fujita, S., Cadenas, J.G., Chinkes, D.L., Volpi, E., and Rasmussen, B.B. 2006. Resistance exercise increases AMPK activity and reduces 4E-BP1 phosphorylation and protein synthesis in human skeletal muscle. J Physiol (Lond) Epub ahead of print: 2006.113175.

Du, J., Wang, X., Miereles, C., Bailey, J.L., Debigare, R., Zheng, B., Price, S.R., and Mitch, W.E. 2004. Activation of caspase-3 is an initial step triggering accelerated muscle proteolysis in catabolic conditions. J. Clin. Invest. 113: 115-123.

Dudley, G.A., and Djamil, R. 1985. Incompatibility of endurance- and strength-training modes of exercise. J Appl Physiol 59: 1446-1451.

Dunn, S.E., Burns, J.L., and Michel, R.N. 1999. Calcineurin Is Required for Skeletal Muscle Hypertrophy 10.1074/jbc.274.31.21908. J. Biol. Chem. 274: 21908-21912.

Dunn, S.E., Chin, E.R., and Michel, R.N. 2000. Matching of Calcineurin Activity to Upstream Effectors Is Critical for Skeletal Muscle Fiber Growth 10.1083/jcb.151.3.663. J. Cell Biol. 151: 663-672.

Dupont-Versteegden, E.E., Fluckey, J.D., Knox, M., Gaddy, D., and Peterson, C.A. 2006. Effect of flywheel-based resistance exercise on processes contributing to muscle atrophy during unloading in adult rats. $J$ Appl Physiol 101: 202-212.

Duran, A., Diaz-Meco, M., and Moscat, J. 2003. Essential role of RelA Ser311 phosphorylation by zetaPKC in NF-kappaB transcriptional activation. $E M B O J$ 22: 3910-3918.

Durante, P.E., Mustard, K.J., Park, S.-H., Winder, W.W., and Hardie, D.G. 2002. Effects of endurance training on activity and expression of AMP-activated protein kinase isoforms in rat muscles. Am J Physiol Endocrinol Metab 283: E178-186.

Durham, W.J., Li, Y.-P., Gerken, E., Farid, M., Arbogast, S., Wolfe, R.R., and Reid, M.B. 2004. Fatiguing exercise reduces DNA binding activity of NF- $\kappa B$ in skeletal muscle nuclei. J Appl Physiol 97: 1740-1745.

Eliasson, J., Elfegoun, T., Nilsson, J., Kohnke, R., Ekblom, B.T., and Blomstrand, E. 2006. Maximal lengthening contractions increase p70S6 kinase phosphorylation in human skeletal muscle in the absence of nutritional supply. Am J Physiol Endocrinol Metab Epub ahead of print: 00141.02006.

Evans, W.J., Phinney, S.D., and Young, V.R. 1982. Suction applied to a muscle biopsy maximizes sample size. Med Sci Sports Exerc 14: 101-102.

Farrell, P.A., Hernandez, J.M., Fedele, M.J., Vary, T.C., Kimball, S.R., and Jefferson, L.S. 2000. Eukaryotic initiation factors and protein synthesis after resistance exercise in rats. J Appl Physiol 88: 1036-1042.

Ferguson, R.A., Ball, D., Krustrup, P., Aagaard, P., Kjar, M., Sargeant, A.J., Hellsten, Y., and Bangsbo, J. 2001. Muscle oxygen uptake and energy turnover during dynamic exercise at different contraction frequencies in humans. $J$ Physiol (Lond) 536: 261271.

Fernandez-Celemin, L., Pasko, N., Blomart, V., and Thissen, J.-P. 2002. Inhibition of muscle insulin-like growth factor I expression by tumor necrosis factor- $\alpha$. Am J Physiol Endocrinol Metab 283: E1279-1290.

Finck, B.N., and Kelly, D.P. 2006. PGC-1 coactivators: inducible regulators of energy metabolism in health and disease. J. Clin. Invest. 116: 615-622.

Flück, M., and Hoppeler, H. 2003. Molecular basis of skeletal muscle plasticity-from gene to form and function. Rev Physiol Biochem Pharmacol 146: 159-216. 
Fluck, M., Waxham, M.N., Hamilton, M.T., and Booth, F.W. 2000. Skeletal muscle Ca2+independent kinase activity increases during either hypertrophy or running. $J$ Appl Physiol 88: 352-358.

Foulstone, E.J., Huser, C., Crown, A.L., Holly, J.M.P., and Stewart, C.E.H. 2004. Differential signalling mechanisms predisposing primary human skeletal muscle cells to altered proliferation and differentiation: roles of IGF-I and TNFa. Exp Cell Res 294: 223-235.

Freyssenet, D., Irrcher, I., Connor, M.K., Di Carlo, M., and Hood, D.A. 2004. Calciumregulated changes in the mitochondrial phenotype in skeletal muscle cells. $\mathrm{Am} J$ Physiol Cell Physiol 286: C1053-1061.

Fritz, T., Kramer, D.K., Karlsson, H.K., Galuska, D., Engfeldt, P., Zierath, J.R., and Krook, A. 2006. Low-intensity exercise increases skeletal muscle protein expression of PPAR $\delta$ and UCP3 in type 2 diabetic patients. Diabetes Metab Res Rev Epub ahead of print.

Frøsig, C., Jørgensen, S.B., Hardie, D.G., Richter, E.A., and Wojtaszewski, J.F.P. 2004. 5'AMP-activated protein kinase activity and protein expression are regulated by endurance training in human skeletal muscle. Am J Physiol Endocrinol Metab 286: E411-E417.

Fry, A.C., Schilling, B.K., Staron, R.S., Hagerman, F.C., Hikida, R.S., and Thrush, J.T. 2003. Muscle fiber characteristics and performance correlates of male Olympic-style weightlifters. J Strength Cond Res 17: 746-754.

Fujii, N., Hayashi, T., Hirshman, M.F., Smith, J.T., Habinowski, S.A., Kaijser, L., Mu, J., Ljungqvist, O., Birnbaum, M.J., and Witters, L.A. 2000. Exercise Induces IsoformSpecific Increase in 5'AMP-Activated Protein Kinase Activity in Human Skeletal Muscle. Biochemical and Biophysical Research Communications 273: 1150-1155.

Funai, K., Parkington, J.D., Carambula, S., and Fielding, R.A. 2006. Age-associated decrease in contraction-induced activation of downstream targets of Akt/mTor signaling in skeletal muscle 10.1152/ajpregu.00277.2005. Am J Physiol Regul Integr Comp Physiol 290: R1080-1086.

Galetic, I., Maira, S.-M., Andjelkovic, M., and Hemmings, B.A. 2003. Negative regulation of ERK and Elk by protein kinase B modulates c-fos transcription. J Biol Chem 278: 4416-4423.

Gao, T., Furnari, F., and Newton, A.C. 2005. PHLPP: A Phosphatase that Directly Dephosphorylates Akt, Promotes Apoptosis, and Suppresses Tumor Growth. Molecular Cell 18: 13-24.

Gao, Z., Hwang, D., Bataille, F., Lefevre, M., York, D., Quon, M.J., and Ye, J. 2002. Serine Phosphorylation of Insulin Receptor Substrate 1 by Inhibitor $\kappa \mathrm{B}$ Kinase Complex. $J$. Biol. Chem. 277: 48115-48121.

Garami, A., Zwartkruis, F.J.T., Nobukuni, T., Joaquin, M., Roccio, M., Stocker, H., Kozma, S.C., Hafen, E., Bos, J.L., and Thomas, G. 2003. Insulin Activation of Rheb, a Mediator of mTOR/S6K/4E-BP Signaling, Is Inhibited by TSC1 and 2. Molecular Cell 11: 1457-1466.

Garcia-Martinez, C., Agell, N., Llovera, M., Lopez-Soriano, F.J., and Argiles, J.M. 1993. Tumour necrosis factor- $\alpha$ increases the ubiquitinization of rat skeletal muscle proteins. FEBS Letters 323: 211-214.

Garcia-Martinez, C., Llovera M., Agell N., Lopezsoriano F. J., and Argiles J. M. 1994. Ubiquitin Gene Expression in Skeletal Muscle Is Increased by Tumor Necrosis Factora. Biochemical and Biophysical Research Communications 201: 682-686.

Gavin, T.P., Robinson, C.B., Yeager, R.C., England, J.A., Nifong, L.W., and Hickner, R.C. 2004. Angiogenic growth factor response to acute systemic exercise in human skeletal muscle. J Appl Physiol 96: 19-24.

Glass, D.J. 2003. Signalling pathways that mediate skeletal muscle hypertrophy and atrophy. Nat Cell Biol 5: 87-90. 
Glass, D.J. 2005. Skeletal muscle hypertrophy and atrophy signaling pathways. Int J Biochem Cell Biol 37: 1974-1984.

Gleyzer, N., Vercauteren, K., and Scarpulla, R.C. 2005. Control of Mitochondrial Transcription Specificity Factors (TFB1M and TFB2M) by Nuclear Respiratory Factors (NRF-1 and NRF-2) and PGC-1 Family Coactivators. Mol. Cell. Biol. 25: 1354-1366.

Glowacki, S., Martin, S., Maurer, A., Baek, W., Green, J., and Crouse, S. 2004. Effects of Resistance, Endurance, and Concurrent Exercise on Training Outcomes in Men. Med Sci Sports Exerc 36: 2119-2127.

Goffart, S., and Wiesner, R.J. 2003. Regulation and co-ordination of nuclear gene expression during mitochondrial biogenesis. Exp Physiol 88: 33-40.

Goldberg, A.L. 1968. Protein synthesis during work-induced growth of skeletal muscle. J Cell Biol 36: 653-658.

Goldspink, G. 2003. Gene expression in muscle in response to exercise. J Muscle Res Cell Motil 24: 121-126.

Gomes, M.D., Lecker, S.H., Jagoe, R.T., Navon, A., and Goldberg, A.L. 2001. Atrogin-1, a muscle-specific F-box protein highly expressed during muscle atrophy. PNAS 98: 14440-14445.

Gordon, J.W., Rungi, A.A., Inagaki, H., and Hood, D.A. 2001. Plasiticity in Skeletal, Cardiac and Smooth Muscle Selected Contribution: Effects of contractile activity on mitochondrial transcription factor A expression in skeletal muscle. J Appl Physiol 90: 389-396.

Grifone, R., Laclef, C., Spitz, F., Lopez, S., Demignon, J., Guidotti, J.-E., Kawakami, K., Xu, P.-X., Kelly, R., Petrof, B.J., et al. 2004. Six1 and Eya1 expression can reprogram adult muscle from the slow-twitch phenotype into the fast-twitch phenotype. Mol Cell Biol 24: 6253-6267.

Haddad, F., and Adams, G.R. 2002. Exercise effects on muscle insulin signaling and action. Selected contribution: Acute cellular and molecular responses to resistance exercise. $J$ Appl Physiol 93: 394-403.

Haddad, F., Adams, G.R., Bodell, P.W., and Baldwin, K.M. 2006. Isometric resistance exercise fails to counteract skeletal muscle atrophy processes during the initial stages of unloading. J Appl Physiol 100: 433-441.

Hahn-Windgassen, A., Nogueira, V., Chen, C.-C., Skeen, J.E., Sonenberg, N., and Hay, N. 2005. Akt Activates the Mammalian Target of Rapamycin by Regulating Cellular ATP Level and AMPK Activity 10.1074/jbc.M502876200. J. Biol. Chem. 280: 3208132089.

Häkkinen, K. 1989. Neuromuscular and hormonal adaptations during strength and power training: A review. J Sports Med Phys Fitness 29: 9-26.

Häkkinen, K., Alen, M., Kraemer, W.J., Gorostiaga, E., Izquierdo, M., Rusko, H., Mikkola, J., Häkkinen, A., Valkeinen, H., Kaarakainen, E., et al. 2003. Neuromuscular adaptations during concurrent strength and endurance training versus strength training. Eur J Appl Physiol 89: 42-52.

Hameed, M., Orrell, R.W., Cobbold, M., Goldspink, G., and Harridge, S.D.R. 2003. Expression of IGF-I splice variants in young and old human skeletal muscle after high resistance training. $J$ Physiol 547: 247-254.

Hannan, K.M., Brandenburger, Y., Jenkins, A., Sharkey, K., Cavanaugh, A., Rothblum, L., Moss, T., Poortinga, G., McArthur, G.A., Pearson, R.B., et al. 2003. mTORDependent Regulation of Ribosomal Gene Transcription Requires S6K1 and Is Mediated by Phosphorylation of the Carboxy-Terminal Activation Domain of the Nucleolar Transcription Factor UBF. Mol. Cell. Biol. 23: 8862-8877. 
Hansen, A.K., Fischer, C.P., Plomgaard, P., Andersen, J.L., Saltin, B., and Pedersen, B.K. 2005. Skeletal muscle adaptation: training twice every second day vs. training once daily. J Appl Physiol 98: 93-99.

Hardie, D.G., and Sakamoto, K. 2006. AMPK: A Key Sensor of Fuel and Energy Status in Skeletal Muscle 10.1152/physiol.00044.2005. Physiology 21: 48-60.

Hardie, D.G., Salt, I.P., Hawley, S.A., and Davies, S.P. 1999. AMP-activated protein kinase: an ultrasensitive system for monitoring cellular energy charge. Biochem $J$ 338: 717 722.

Harrington, L.S., Findlay, G.M., and Lamb, R.F. 2005. Restraining PI3K: mTOR signalling goes back to the membrane. Trends in Biochemical Sciences 30: 35-42.

Hawke, T.J., and Garry, D.J. 2001. Myogenic satellite cells: physiology to molecular biology. J Appl Physiol 91: 534-551.

Hawley, J., Tipton, K., and Millard-Stafford, M. 2006. Promoting training adaptations through nutritional interventions. J Sports Sci 24: 709-721.

Hawley, J.A. 2002. Adaptations of skeletal muscle to prolonged, intense endurance training. Clin Exp Pharmacol Physiol 29: 218-222.

Hawley, J.A., and Noakes, T.D. 1992. Peak power output predicts maximal oxygen uptake and performance time in trained cyclists. Eur J Appl Physiol Occup Physiol 65: 79-83.

Hawley, J.A., and Zierath, J. 2004. Integration of metabolic and mitogenic signal transduction in skeletal muscle. Exerc Sport Sci Rev 32: 4-8.

Hawley, S.A., Pan, D.A., Mustard, K.J., Ross, L., Bain, J., Edelman, A.M., Frenguelli, B.G., and Hardie, D.G. 2005. Calmodulin-dependent protein kinase kinase- $\beta$ is an alternative upstream kinase for AMP-activated protein kinase. Cell Metabolism 2: 919.

Hayashi, T., Hirshman, M., Kurth, E., Winder, W., and Goodyear, L. 1998. Evidence for 5' AMP-activated protein kinase mediation of the effect of muscle contraction on glucose transport. Diabetes 47: 1369-1373.

Hennessy, L.C., and Watson, A.W.S. 1994. The interference effects of training for strength and endurance simultaneously. J Strength Cond Res 8: 12-19.

Heszele, M.F.C., and Price, S.R. 2004. Insulin-Like Growth Factor I: The Yin and Yang of Muscle Atrophy 10.1210/en.2004-1037. Endocrinology 145: 4803-4805.

Hickson, R.C. 1980. Interference of strength development by simultaneously training for strength and endurance. Eur J Appl Physiol Occup Physiol 45: 255-263.

Higginson, J., Wackerhage, H., Woods, N., Schjerling, P., Ratkevicius, A., Grunnet, N., and Quistorff, B. 2002. Blockades of mitogen-activated protein kinase and calcineurin both change fibre-type markers in skeletal muscle culture. Pflugers Arch 445: 437443.

Hildebrandt, A.L., Pilegaard, H., and Neufer, P.D. 2003. Differential transcriptional activation of select metabolic genes in response to variations in exercise intensity and duration in red and white skeletal muscle. Am J Physiol Endocrinol Metab Epub ahead of print: 00234.02003.

Hintz, C.S., Chi, M.M., Fell, R.D., Ivy, J.L., Kaiser, K.K., Lowry, C.V., and Lowry, O.H. 1982. Metabolite changes in individual rat muscle fibers during stimulation. $A m J$ Physiol Cell Physiol 242: C218-228.

Hishiya, A., Iemura, S., Natsume, T., Takayama, S., Ikeda, K., and Watanabe, K. 2006. A novel ubiquitin-binding protein ZNF216 functioning in muscle atrophy. EMBO J. 25: 554-564.

Ho, R.C., Hirshman, M.F., Li, Y., Cai, D., Farmer, J.R., Aschenbach, W.G., Witczak, C.A.,

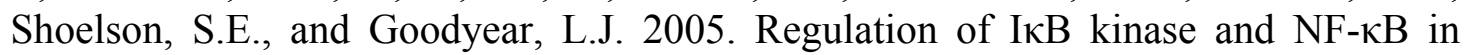
contracting adult rat skeletal muscle. Am J Physiol Cell Physiol 289: C794-801.

Hoffman, E.P., and Nader, G.A. 2004. Balancing muscle hypertrophy and atrophy. Nat Med 10: $584-585$. 
Holloszy, J.O. 1967. Biochemical adaptations in muscle. Effects of exercise on mitochondrial oxygen uptake and respiratory enzyme activity in skeletal muscle. J Biol Chem 242: 2278-2282.

Holloszy, J.O., and Coyle, E.F. 1984. Adaptations of skeletal muscle to endurance exercise and their metabolic consequences. J Appl Physiol 56: 831-838.

Holloszy, J.O., Rennie, M.J., Hickson, R.C., Conlee, R.K., and Hagberg, J.M. 1977. Physiological consequences of the biochemical adaptations to endurance exercise. Ann N Y Acad Sci 301: 440-450.

Holloway, G.P., Green, H.J., Duhamel, T.A., Ferth, S., Moule, J.W., Ouyang, J., and Tupling, A.R. 2005. Muscle sarcoplasmic reticulum $\mathrm{Ca} 2+$ cycling adaptations during $16 \mathrm{~h}$ of heavy intermittent cycle exercise 10.1152/japplphysiol.01407.2004. J Appl Physiol 99: 836-843.

Hood, D.A. 2001. Plasticity in Skeletal, Cardiac, and Smooth Muscle Invited Review: Contractile activity-induced mitochondrial biogenesis in skeletal muscle. $\mathrm{J} \mathrm{Appl}$ Physiol 90: 1137-1157.

Hood, D.A., Irrcher, I., Ljubicic, V., and Joseph, A.-M. 2006. Coordination of metabolic plasticity in skeletal muscle. J Exp Biol 209: 2265-2275.

Hoppeler, H., and Flück, M. 2003. Plasticity of skeletal muscle mitochondria: Structure and function. Med Sci Sports Exerc 35: 95-104.

Horman, S., Browne, G.J., Krause, U., Patel, J.V., Vertommen, D., Bertrand, L., Lavoinne, A., Hue, L., Proud, C.G., and Rider, M.H. 2002. Activation of AMP-Activated Protein Kinase Leads to the Phosphorylation of Elongation Factor 2 and an Inhibition of Protein Synthesis. Current Biology 12: 1419-1423.

Hornberger, T., Stuppard, R., Conley, K., Fedele, M., Fiorotto, M., Chin, E., and Esser, K.A. 2004. Mechanical stimuli regulate rapamycin-sensitive signalling by a phosphoinositide 3-kinase-, protein kinase B- and growth factor-independent mechanism. Biochem J 380(pt3): 795-804.

Hornberger, T.A., Armstrong, D.D., Koh, T.J., Burkholder, T.J., and Esser, K.A. 2005a. Intracellular signaling specificity in response to uniaxial vs. multiaxial stretch: implications for mechanotransduction 10.1152/ajpcell.00207.2004. Am J Physiol Cell Physiol 288: C185-194.

Hornberger, T.A., and Esser, K.A. 2004. Mechanotransduction and the regulation of protein synthesis in skeletal muscle. Proc Nutr Soc 63: 331-335.

Hornberger, T.A., Mateja, R.D., Chin, E.R., Andrews, J.L., and Esser, K.A. 2005b. Aging does not alter the mechanosensitivity of the p38, p70S6k, and JNK2 signaling pathways in skeletal muscle 10.1152/japplphysiol.00870.2004. J Appl Physiol 98: $1562-1566$.

Horne, L., Bell, G., Fisher, B., Warren, S., and Janowska-Wieczorek, A. 1997. Interaction between cortisol and tumour necrosis factor with concurrent resistance and endurance training. Clin J Sport Med 7: 247-251.

Houston, M.E. 2001. Biochemistry primer for exercise science, 2nd ed. Human Kinetics, Campaign, Il.

Houten, S.M., and Auwerx, J. 2004. Turbocharging mitochondria. Cell 119: 5-7.

Hresko, R.C., and Mueckler, M. 2005. mTOR RICTOR Is the Ser473 Kinase for Akt/Protein Kinase B in 3T3-L1 Adipocytes 10.1074/jbc.M508361200. J. Biol. Chem. 280: 40406-40416.

Hudson, E.R., Pan, D.A., James, J., Lucocq, J.M., Hawley, S.A., Green, K.A., Baba, O., Terashima, T., and Hardie, D.G. 2003. A Novel Domain in AMP-Activated Protein Kinase Causes Glycogen Storage Bodies Similar to Those Seen in Hereditary Cardiac Arrhythmias. Curr Biol 13: 861-866. 
Hunter, G., Demment, R., and Miller, D. 1987. Development of strength and maximum oxygen uptake during simultaneous training for strength and endurance. J Sports Med Phys Fitness 27: 269-275.

Hunter, R.B., Stevenson, E., Koncarevic, A., Mitchell-Felton, H., Essig, D.A., and Kandarian, S.C. 2002. Activation of an alternative NF- $\kappa \mathrm{B}$ pathway in skeletal muscle during disuse atrophy. Faseb $J$ 16: 529-538.

Hurley, R.L., Anderson, K.A., Franzone, J.M., Kemp, B.E., Means, A.R., and Witters, L.A. 2005. The $\mathrm{Ca} 2+/$ Calmodulin-dependent Protein Kinase Kinases Are AMP-activated Protein Kinase Kinases 10.1074/jbc.M503824200. J. Biol. Chem. 280: 29060-29066.

Hurst, D., Taylor, E.B., Cline, T.D., Greenwood, L.J., Compton, C.L., Lamb, J.D., and Winder, W.W. 2005. AMP-activated protein kinase kinase activity and phosphorylation of AMP-activated protein kinase in contracting muscle of sedentary and endurance-trained rats. Am J Physiol Endocrinol Metab 289: E710-715.

Ihlemann, J., Ploug, T., Hellsten, Y., and Galbo, H. 1999. Effect of tension on contractioninduced glucose transport in rat skeletal muscle. Am J Physiol Endocrinol Metab 277: E208-214.

Ingalls, C.P. 2004. Nature vs. nurture: can exercise really alter fibre type composition in human skeletal muscle. J Appl Physiol 97: 1591-1592.

Inoki, K., Li, Y., Zhu, T., Wu, J., and Guan, K.L. 2002. TSC2 is phosphorylated and inhibited by Akt and suppresses mTOR signalling. Nat Cell Biol 4: 648-657.

Irrcher, I., Adhihetty, P.J., Joseph, A.M., Ljubicic, V., and Hood, D.A. 2003. Regulation of mitochondrial biogenesis in muscle by endurance exercise. Sports Med 33: 783-793.

Irrcher, I., and Hood, D.A. 2004. Regulation of Egr-1, SRF, and Sp1 mRNA expression in contracting skeletal muscle cells. J Appl Physiol 97: 2207-2213.

Ishido, M., Kami, K., and Masuhara, M. 2004a. In vivo expression patterns of MyoD, p21, and $\mathrm{Rb}$ proteins in myonuclei and satellite cells of denervated rat skeletal muscle. $\mathrm{Am}$ J Physiol Cell Physiol 287: C484-493.

Ishido, M., Kami, K., and Masuhara, M. 2004b. Localization of MyoD, myogenin and cell cycle regulatory factors in hypertrophying skeletal muscle. Acta Physiol Scand 180: 281-289.

Ivy, J.L., Chi, M.M., Hintz, C.S., Sherman, W.M., Hellendall, R.P., and Lowry, O.H. 1987. Progressive metabolite changes in individual human muscle fibers with increasing work rates. Am J Physiol Cell Physiol 252: C630-639.

Izquierdo, M., Hakkinen, K., Ibanez, J., Kraemer, W.J., and Gorostiaga, E.M. 2005. Effects of combined resistance and cardiovascular training on strength, power, muscle crosssectional area, and endurance markers in middle-aged men. Eur J Appl Physiol 94: 7075.

Izquierdo, M., Ibanez, J., Hakkinen, K., Kraemer, W.J., Ruesta, M., and Gorostiaga, E.M. 2004. Maximal strength and power, muscle mass, endurance and serum hormones in weightlifters and road cyclists. J Sports Sci 22: 465-478.

Jackman, R.W., and Kandarian, S.C. 2004. The molecular basis of skeletal muscle atrophy. Am J Physiol Cell Physiol 287: C834-843.

Jacquemin, V., Furling, D., Bigot, A., Butler-Browne, G.S., and Mouly, V. 2004. IGF-1 induces human myotube hypertrophy by increasing cell recruitment. Experimental Cell Research 299: 148-158.

Jefferson, L.S., Fabian, J.R., and Kimball, S.R. 1999. Glycogen synthase kinase-3 is the predominant insulin-regulated eukaryotic initiation factor $2 \mathrm{~B}$ kinase in skeletal muscle. The International Journal of Biochemistry \& Cell Biology 31: 191-200.

Jemiolo, B., and Trappe, S. 2004. Single muscle fiber gene expression in human skeletal muscle: validation of internal control with exercise. Biochem Biophys Res Commun 320: $1043-1050$. 
Ji, L.L., Gomez-Cabrera, M.C., Steinhafel, N., and Vina, J. 2004. Acute exercise activates nuclear factor NF-kB signaling pathway in rat skeletal muscle. Faseb $J$ 18: 14991506.

Jindra, M., Gaziova, I., Uhlirova, M., Okabe, M., Hiromi, Y., and Hirose, S. 2004. Coactivator MBF1 preserves the redox-dependent AP-1 activity during oxidative stress in Drosophila. EMBO J 23: 3538-3547.

Jones, N.C., Tyner, K.J., Nibarger, L., Stanley, H.M., Cornelison, D.D.W., Fedorov, Y.V., and Olwin, B.B. 2005. The p38 $\alpha / \beta$ MAPK functions as a molecular switch to activate the quiescent satellite cell. J. Cell Biol. 169: 105-116.

Jones, S.W., Hill, R.J., Krasney, P.A., O'Conner, B., Peirce, N., and Greenhaff, P.L. 2004. Disuse atrophy and exercise rehabilitation in humans profoundly affects the expression of genes associated with regulation of skeletal muscle mass. FASEB $J$ 18: 1025-1027.

Jorgensen, S.B., Wojtaszewski, J.F.P., Viollet, B., Andreelli, F., Birk, J.B., Hellsten, Y., Schjerling, P., Vaulont, S., Neufer, P.D., Richter, E.A., et al. 2005. Effects of $\alpha$ AMPK knockout on exercise-induced gene activation in mouse skeletal muscle. FASEB J.: 04-3144fje.

Kadi, F., Johansson, F., Johansson, R., Sjöström, M., and Henriksson, J. 2004. Effects of one bout of endurance exercise on the expresion of myogenin in human quadriceps muscle. Histochem Cell Biol 121: 329-334.

Kandarian, S., and Jackman, R. 2006. Intracellular signaling during skeletal muscle atrophy. Muscle Nerve 33: 155-165.

Kanki, T., Ohgaki, K., Gaspari, M., Gustafsson, C.M., Fukuoh, A., Sasaki, N., Hamasaki, N., and Kang, D. 2004. Architectural Role of Mitochondrial Transcription Factor A in Maintenance of Human Mitochondrial DNA. Mol. Cell. Biol. 24: 9823-9834.

Karlsson, H.K.R., Nilsson, P.-A., Nilsson, J., Chibalin, A.V., Zierath, J.R., and Blomstrand, E. 2004. Branched-chain amino acids increase p70S6k phosphorylation in human skeletal muscle after resistance exercise. Am J Physiol Endocrinol Metab 287: E1-7.

Kaushik, V.K., Young, M.E., Dean, D.J., Kurowski, T.G., Saha, A.K., and Ruderman, N.B. 2001. Regulation of fatty acid oxidation and glucose metabolism in rat soleus muscle: effects of AICAR. Am J Physiol Endocrinol Metab 281: E335-340.

Kearns, J.D., Basak, S., Werner, S.L., Huang, C.S., and Hoffmann, A. 2006. IкB $\varepsilon$ provides negative feedback to control NF- $\mathrm{BB}$ oscillations, signaling dynamics, and inflammatory gene expression. J. Cell Biol. 173: 659-664.

Keren, A., Tamir, Y., and Bengal, E. 2006. The p38 MAPK signaling pathway: A major regulator of skeletal muscle development. Molecular and Cellular Endocrinology In Press, Corrected Proof.

Kim, D.-H., Sarbassov, D.D., Ali, S.M., Latek, R.R., Guntur, K.V.P., Erdjument-Bromage, H., Tempst, P., and Sabatini, D.M. 2003. GßL, a Positive Regulator of the RapamycinSensitive Pathway Required for the Nutrient-Sensitive Interaction between Raptor and mTOR. Molecular Cell 11: 895-904.

Kim, J.-S., Cross, J.M., and Bamman, M.M. 2005. Impact of resistance loading on myostatin expression and cell cycle regulation in young and older men and women. Am J Physiol Endocrinol Metab 288: E1110-1119.

Kimura, N., Tokunaga, C., Dalal, S., Richardson, C., Yoshino, K.-I., Hara, K., Kemp, B.E., Witters, L.A., Mimura, O., and Yonezawa, K. 2003. A possible linkage between AMP-activated protein kinase (AMPK) and mammalian target of rapamycin (mTOR) signalling pathway. Genes Cells 8: 65-79.

Koopman, R., Zorenc, A.H.G., Gransier, R.J.J., Cameron-Smith, D., and van Loon, L.J.C. 2006. Increase in S6K1 phosphorylation in human skeletal muscle following resistance exercise occurs mainly in type II muscle fibers 10.1152/ajpendo.00530.2005. Am J Physiol Endocrinol Metab 290: E1245-1252. 
Kosek, D.J., Kim, J.-s., Petrella, J.K., Cross, J.M., and Bamman, M.M. 2006. Efficacy of 3 $\mathrm{D} / \mathrm{WK}$ resistance training on myofiber hypertrophy and myogenic mechanisms in young versus older adults. $J$ Appl Physiol Epub ahead of print: 01474.02005.

Kostek, M.C., Delmonico, M.J., Reichel, J.B., Roth, S.M., Douglass, L., Ferrell, R.E., and Hurley, B.F. 2005. Muscle strength response to strength training is influenced by insulin-like growth factor 1 genotype in older adults. $J$ Appl Physiol 98: 2147-2154.

Kostyak, J.C., Kimball, S.R., Jefferson, L.S., and Farrell, P.A. 2001. Severe diabetes inhibits resistance exercise-induced increase in eukaryotic initiation factor $2 \mathrm{~B}$ activity. $J \mathrm{Appl}$ Physiol 91: 79-84.

Koulmann, N., and Bigard, A. 2006. Interaction between signalling pathways involved in skeletal muscle responses to endurance exercise. Pflugers Arch 452: 125-139.

Krisan, A.D., Collins, D.E., Crain, A.M., Kwong, C.C., Singh, M.K., Bernard, J.R., and Yaspelkis, B.B., III. 2004. Resistance training enhances components of the insulin signaling cascade in normal and high-fat-fed rodent skeletal muscle. J Appl Physiol 96: $1691-1700$.

Krook, A., Widegren, U., Jiang, X.J., Henriksson, J., Wallberg-Henriksson, H., Alessi, D., and Zierath, J.R. 2000. Effects of exercise on mitogen- and stress-activated signal transduction in human skeletal muscle. Am J Physiol Regul Integr Comp Physiol 279: R1716-R1721.

Krustrup, P., Ferguson, R.A., Kjar, M., and Bangsbo, J. 2003. ATP and heat production in human skeletal muscle during dynamic exercise: higher efficiency of anaerobic than aerobic ATP resynthesis 10.1113/jphysiol.2002.035089. J Physiol (Lond) 549: 255269.

Kubica, N., Bolster, D.R., Farrell, P.A., Kimball, S.R., and Jefferson, L.S. 2005. Resistance Exercise Increases Muscle Protein Synthesis and Translation of Eukaryotic Initiation Factor 2B $\varepsilon$ mRNA in a Mammalian Target of Rapamycin-dependent Manner. J Biol Chem 280: 7570-7580.

Kubica, N., Kimball, S.R., Jefferson, L.S., and Farrell, P.A. 2004. Alterations in the expression of mRNAs and proteins that code for species relevant to eIF2B activity after an acute bout of resistance exercise 10.1152/japplphysiol.00962.2003. J Appl Physiol 96: 679-687.

Kumar, A., and Boriek, A.M. 2003. Mechanical stress activates the nuclear factor-kappaB pathway in skeletal muscle fibers: a possible role in Duchenne muscular dystrophy. Faseb J 17: 386-396.

Kumar, A., Chaudhry, I., Reid, M.B., and Boriek, A.M. 2002. Distinct Signaling Pathways Are Activated in Response to Mechanical Stress Applied Axially and Transversely to Skeletal Muscle Fibers 10.1074/jbc.M203654200. J. Biol. Chem. 277: 46493-46503.

Lai, K.-M.V., Gonzalez, M., Poueymirou, W.T., Kline, W.O., Na, E., Zlotchenko, E., Stitt, T.N., Economides, A.N., Yancopoulos, G.D., and Glass, D.J. 2004. Conditional activation of Akt in adult skeletal muscle induces rapid hypertrophy. Mol Cell Biol 24: 9295-9304.

Lang, C.H., Frost, R.A., Nairn, A.C., MacLean, D.A., and Vary, T.C. 2002. TNF- $\alpha$ impairs heart and skeletal muscle protein synthesis by altering translation initiation. Am J Physiol Endocrinol Metab 282: E336-347.

Lang, C.H., Krawiec, B.J., Huber, D., McCoy, J.M., and Frost, R.A. 2006. Sepsis and inflammatory insults downregulate IGFBP-5, but not IGFBP-4, in skeletal muscle via a TNF-dependent mechanism. Am J Physiol Regul Integr Comp Physiol 290: R963972.

Langen, R.C.J., Van Der Velden, J.L.J., Schols, A.M.W.J., Kelders, M.C.J.M., Wouters, E.F.M., and Janssen-Heinnger, Y.M.W. 2004. Tumor necrosis factor- $\alpha$ inhibits myogenic differentiation through MyoD protein destabilization. FASEB J. 18: 227237. 
Langley, B., Thomas, M., Bishop, A., Sharma, M., Gilmour, S., and Kambadur, R. 2002. Myostatin inhibits myoblast differentiation by down-regulating MyoD expression. $J$ Biol Chem 277: 49831-49840.

Latres, E., Amini, A.R., Amini, A.A., Griffiths, J., Martin, F.J., Wei, Y., Lin, H.C., Yancopoulos, G.D., and Glass, D.J. 2005. Insulin-like Growth Factor-1 (IGF-1) Inversely Regulates Atrophy-induced Genes via the Phosphatidylinositol 3Kinase/Akt/Mammalian Target of Rapamycin (PI3K/Akt/mTOR) Pathway. J. Biol. Chem. 280: 2737-2744.

LeBlanc, P.J., Peters, S.J., Tunstall, R.J., Cameron-Smith, D., and Heigenhauser, G.J.F. 2004. Effect of aerobic training on pyruvate dehydrogenase and pyruvate dehydrogenase kinase in human skeletal muscle. $J$ Physiol 557: 559-570.

Lecker, S.H., Jagoe, R.T., Gilbert, A., Gomes, M., Baracos, V., Bailey, J., Price, S.R., Mitch, W.E., and Goldberg, A.L. 2004. Multiple types of skeletal muscle atrophy involve a common program of changes in gene expression. FASEB J. 18: 39-51.

Lecker, S.H., Solomon, V., Mitch, W.E., and Goldberg, A.L. 1999. Muscle Protein Breakdown and the Critical Role of the Ubiquitin-Proteasome Pathway in Normal and Disease States. J. Nutr. 129: 227S-.

Lee, C.-H., Olson, P., and Evans, R.M. 2003. Minireview: Lipid Metabolism, Metabolic Diseases, and Peroxisome Proliferator-Activated Receptors. Endocrinology 144: 2201-2207.

Lee, J.L., Kim, M., Park, H.-S., Kim, H.S., Jeon, M.J., Oh, K.S., Koh, E.H., Won, J.C., Kim, M.-S., Oh, G.T., et al. 2006. AMPK activation increases fatty acid oxidation in skeletal muscle by activating PPAR $\alpha$ and PGC-1. Biochem Biophys Res Commun 340: 291-295.

Lee, S.-J., and McPherron, A.C. 2001. Regulation of myostatin activity and muscle growth. PNAS 98: 9306-9311.

Leveritt, M.D., Abernethy, P.J., Barry, B., and Logan, P.A. 2003. Concurrent strength and endurance training: the influence of dependent variable selection. J Strength Cond Res 17: 503-508.

Leveritt, M.D., Abernethy, P.J., Barry, B.K., and Logan, P.A. 1999. Concurrent strength and endurance training: A review. Sports Med 28: 413-427.

Li, P., Akimoto, T., Zhang, M., Williams, R.S., and Yan, Z. 2006. Resident stem cells are not required for exercise-induced fiber-type switching and angiogenesis but are necessary for activity-dependent muscle growth. Am J Physiol Cell Physiol 290: C1461-1468.

Li, Y.-P., Chen, Y., John, J., Moylan, J., Jin, B., Mann, D.L., and Reid, M.B. 2005. TNF- $\alpha$ acts via p38 MAPK to stimulate expression of the ubiquitin ligase atrogin1/MAFbx in skeletal muscle. FASEB J. 19: 362-370.

Li, Y.-P., LECKER, S.H., CHEN, Y., WADDELL, I.D., GOLDBERG, A.L., and REID, M.B. 2003. TNF- $\alpha$ increases ubiquitin-conjugating activity in skeletal muscle by upregulating $\mathrm{UbcH} 2 / \mathrm{E} 220 \mathrm{k}$. FASEB J. 17: 1048-1057.

Lin, J., Wu, H., Tarr, P.T., Zhang, C.-Y., Wu, Z., Boss, O., Michael, L.F., Puigserver, P., Isotani, E., Olson, E.N., et al. 2002. Transcriptional co-activator PGC-1 $\alpha$ drives the formation of slow-twitch muscle fibres. Nature 418: 797-801.

Lin, S.-J., and Guarente, L. 2003. Nicotinamide adenine dinucleotide, a metabolic regulator of transcription, longevity and disease. Current Opinion in Cell Biology 15: 241-246.

Liu, Y., Shen, T., Randall, W.R., and Schneider, M.F. 2005. Signaling pathways in activitydependent fiber type plasticity in adult skeletal muscle. J Muscle Res Cell Motil 26: 13-21.

Lluis, F., Ballestar, E., Suelves, M., Esteller, M., and Munoz-Canoves, P. 2005. E47 phosphorylation by 38 MAPK promotes MyoD/E47 association and muscle-specific gene transcription. EMBO J 24: 974-984. 
Lluis, F., Perdiguero, E., Nebreda, A.R., and Munoz-Canoves, P. 2006. Regulation of skeletal muscle gene expression by p38 MAP kinases. Trends in Cell Biology 16: 36-44.

Loughna, P.T., Izumo, S., Goldspink, G., and Nadal-Ginard, B. 1990. Disuse and passive stretch cause rapid alterations in expression of developmental and adult contractile protein genes in skeletal muscle. Development 109: 217-223.

Loughna, P.T., and Morgan, M.J. 1999. Passive stretch modulates denervation induced alterations in skeletal muscle myosin heavy chain mRNA levels. Pflugers Achiv 439: 52-55.

Lund, S., Pryor, P.R., Ostergaard, S., Schmitz, O., Pedersen, O., and Holman, G.D. 1998. Evidence against protein kinase $\mathrm{B}$ as a mediator of contraction-induced glucose transport and GLUT4 translocation in rat skeletal muscle. FEBS Letters 425: 472-474.

Luquet, S., Lopez-Soriano, J., Holst, D., Fredenrich, A., Melki, J., Rassoulzadegan, M., and Grimaldi, P.A. 2003. Peroxisome proliferator-activated receptor \&delta; controls muscle development and oxydative capability. FASEB J.: 03-0269fje.

Ma, L., Chen, Z., Erdjument-Bromage, H., Tempst, P., and Pandolfi, P.P. 2005. Phosphorylation and Functional Inactivation of TSC2 by Erk: Implications for Tuberous Sclerosis and Cancer Pathogenesis. Cell 121: 179-193.

MacDougall, J.D., Sale, D.G., Elder, G.C., and Sutton, J.R. 1982. Muscle ultrastructural characteristics of elite powerlifters and bodybuilders. Eur J Appl Physiol Occup Physiol 48: 117-126.

MacPherson, P., Dennis, R., and Faulkner, J. 1997. Sarcomere dynamics and contractioninduced injury to maximally activated single muscle fibres from soleus muscles of rats. $J$ Physiol 500: 523-580.

Mahoney, D.J., Carey, K., Fu, M.-H., Snow, R., Cameron-Smith, D., Parise, G., and Tarnopolsky, M.A. 2004. Real-time RT-PCR analysis of housekeeping genes in human skeletal muscle following acute exercise. Physiol Genomics 18: 226-231.

Mahoney, D.J., Parise, G., Melov, S., Safdar, A., and Tarnopolsky, M.A. 2005. Analysis of global mRNA expression in human skeletal muscle during recovery from endurance exercise. FASEB J. 19: 1498-14500.

Maniura-Weber, K., Goffart, S., Garstka, H.L., Montoya, J., and Wiesner, R.J. 2004. Transient overexpression of mitochondrial transcription factor A (TFAM) is sufficient to stimulate mitochondrial DNA transcription, but not sufficient to increase mtDNA copy number in cultured cells. Nucl. Acids Res. 32: 6015-6027.

Manning, B.D., and Cantley, L.C. 2003. Rheb fills a GAP between TSC and TOR. Trends Biochem Sci 28: 573-576.

Markuns, J.F., Wojtaszewski, J.F.P., and Goodyear, L.J. 1999. Insulin and Exercise Decrease Glycogen Synthase Kinase-3 Activity by Different Mechanisms in Rat Skeletal Muscle. J. Biol. Chem. 274: 24896-24900.

Matsakas, A., Friedel, A., Hertrampf, T., and Diel, P. 2005. Short-term endurance training results in a muscle-specific decrease of myostatin mRNA content in the rat. Acta Physiol Scand 183: 299-307.

Matsunaga, S., Inashima, S., Tsuchimochi, H., Yamada, T., Hazama, T., and Wada, M. 2002. Altered sarcoplasmic reticulum function in rat diaphragm after high-intensity exercise. Acta Physiol Scand 176: 227-232.

Matsuzaki, H., Daitoku, H., Hatta, M., Tanaka, K., and Fukamizu, A. 2003. Insulin-induced phosphorylation of FKHR (Foxo1) targets to proteasomal degradation. PNAS 100: 11285-11290.

Matuszczak, Y., Farid, M., Jones, J., Lansdowne, S., Smith, M.A., Taylor, A.A., and Reid, M.B. 2005. Effects of N-acetylcysteineon glutathione oxidation and fatigue during handgrip exercise. Muscle Nerve 32: 633-638. 
McCarthy, J.P., Agre, J.C., Graf, B.K., Pozniak, M.A., and Vailas, A.C. 1995. Compatibility of adaptive responses with combining strength and endurance training. Med Sci Sports Exerc 27: 429-436.

McCarthy, J.P., Pozniak, M.A., and Agre, J.C. 2002. Neuromuscular adaptations to concurrent strength and endurance training. Med Sci Sports Exerc 34: 511-519.

McConell, G.K., Lee-Young, R.S., Chen, Z.-P., Stepto, N.K., Huynh, N.N., Stephens, T.J., Canny, B.J., and Kemp, B.E. 2005. Short-term exercise training in humans reduces AMPK signalling during prolonged exercise independent of muscle glycogen. $J$ Physiol (Lond) 568: 665-676.

McCroskery, S., Thomas, M., Maxwell, L., Sharma, M., and Kambadur, R. 2003. Myostatin negatively regulates satellite cell activation and self-renewal. J. Cell Biol. 162: 11351147.

McCully, K.K., and Faulkner, J.A. 1985. Injury to skeletal muscle fibers of mice following lengthening contractions. $J$ Appl Physiol 59: 119-126.

McGee, S.L., and Hargreaves, M. 2004. Exercise and Myocyte Enhancer Factor 2 Regulation in Human Skeletal Muscle. Diabetes 53: 1208-1214.

McNally, E.M. 2004. Powerful Genes - Myostatin regulation of human muscle mass. $N$ Engl $J$ Med 350: 2642-2644.

McPherron, A.C., and Lee, S.-J. 1997. Double muscling in cattle due to mutations in the myostatin gene. PNAS 94: 12457-12461.

Medved, I., Brown, M.J., Bjorksten, A.R., Leppik, J.A., Sostaric, S., and McKenna, M.J. 2003. N-acetylcysteine infusion alters blood redox status but not time to fatigue during intense exercise in humans 10.1152/japplphysiol.00884.2002. J Appl Physiol 94: 1572-1582.

Medved, I., Brown, M.J., Bjorksten, A.R., Murphy, K.T., Petersen, A.C., Sostaric, S., Gong, X., and McKenna, M.J. 2004. N-acetylcysteine enhances muscle cysteine and glutathione availability and attenuates fatigue during prolonged exercise in endurancetrained individuals 10.1152/japplphysiol.00371.2004. J Appl Physiol 97: 1477-1485.

Merrill, G.F., Kruth, E.J., Hardie, D.G., and Winder, W.W. 1997. AICA riboside increases AMP-activated protein kinase, fatty acid oxidation, and glucose uptake in rat muscle. Am J Physiol Endocrinol Metab 273: E1107-1112.

Michel, R.N., Dunn, S.E., and Chin, E.R. 2004. Calcineurin and skeletal muscle growth. Proc Nutr Soc 63: 341-349.

Millet, G.P., Jaouen, B., Borrani, F., and Candau, R. 2002. Effects of concurrent endurance and strength training on running economy and $\mathrm{VO}_{2}$ kinetics. Med Sci Sports Exerc 34: 1351-1359.

Mitchell, P.O., and Pavlath, G.K. 2004. Skeletal muscle atrophy leads to loss and dysfunction of muscle precursor cells. Am J Physiol Cell Physiol 287: C1753-1762.

Moelling, K., Schad, K., Bosse, M., Zimmermann, S., and Schweneker, M. 2002. Regulation of Raf-Akt cross-talk. J Biol Chem 277: 31099-31106.

Montagne, J., Stewart, M.J., Stocker, H., Hafen, E., Kozma, S.C., and Thomas, G. 1999. Drosophila S6 Kinase: A Regulator of Cell Size. Science 285: 2126-2129.

Murgia, M., Serrano, A.L., Calabria, E., Pallafacchina, G., Lomo, T., and Schiaffino, S. 2000. Ras is involved in nerve-activity-dependent regulation of muscle genes. Nat Cell Biol 2: 142-147.

Murphy, L.O., MacKeigan, J.P., and Blenis, J. 2004. A network of immediate early gene products propogates subtle differences in mitogen-activated protein kinase signal amplitude and duration. Mol Cell Biol 24: 144-153.

Murphy, L.O., Smith, S., Chen, R.-H., Fingar, D.C., and Blenis, J. 2002. Molecular interpretation of ERK signal duration by immediate early gene products. Nat Cell Biol 4: 556-564. 
Murphy, R.M., Watt, K.K.O., Cameron-Smith, D., Gibbons, C.J., and Snow, R.J. 2003. Effects of creatine supplementation on housekeeping genes in human skeletal muscle using real-time RT-PCR. Physiol. Genomics 12: 163-174.

Musaro, A., McCullagh, K., Paul, A., Houghton, L., Dobrowolny, G., Molinaro, M., Barton, E.R., L Sweeney, H., and Rosenthal, N. 2001. Localized Igf-1 transgene expression sustains hypertrophy and regeneration in senescent skeletal muscle. 27: 195-200.

Musaro, A., McCullagh, K.J.A., Naya, F.J., Olson, E.N., and Rosenthal, N. 1999. IGF-1 induces skeletal myocyte hypertrophy through calcineurin in association with GATA2 and NF-ATc1. Nature 400: 581-585.

Musi, N., Hayashi, T., Fujii, N., Hirshman, M.F., Witters, L.A., and Goodyear, L.J. 2001. AMP-activated protein kinase activity and glucose uptake in rat skeletal muscle. $\mathrm{Am} \mathrm{J}$ Physiol Endocrinol Metab 280: E677-684.

Nader, G.A. 2005. Molecular determinants of skeletal muscle mass: getting the "AKT" together. Int J Biochem Cell Biol 37: 1985-1996.

Nader, G.A., and Esser, K.A. 2001. Intracellular signaling specificity in skeletal muscle in response to different modes of exercise. J Appl Physiol 90: 1936-1942.

Nader, G.A., McLoughlin, T.J., and Esser, K.A. 2005. mTOR function in skeletal muscle hypertrophy: increased ribosomal RNA via cell cycle regulators 10.1152/ajpcell.00165.2005. Am J Physiol Cell Physiol 289: C1457-1465.

Nairn, A., and Palfrey, H. 1987. Identification of the major Mr 100,000 substrate for calmodulin- dependent protein kinase III in mammalian cells as elongation factor-2. $J$. Biol. Chem. 262: 17299-17303.

Nakano, Hamada, Hayashi, Yonemitsu, Miyamoto, Toyoda, Tanaka, Masuzaki, Ebihara, Ogawa, et al. 2006. 2 Isoform-specific activation of 5'adenosine monophosphateactivated protein kinase by 5-aminoimidazole-4-carboxamide-1-d-ribonucleoside at a physiological level activates glucose transport and increases glucose transporter 4 in mouse skeletal muscle. Metabolism 55: 300-308.

Natoli, G., Saccani, S., Bosisio, D., and Marazzi, I. 2005. Interactions of NF- $\kappa$ B with chromatin: the art of being at the right place at the right time. Nat Immunol 6: 439445.

Nave, B.T., Ouwens, M., Withers, D.J., Alessi, D.R., and Shepherd, P.R. 1999. Mammalian target of rapamycin is a direct target for protein kinase B: identification of a convergence point for opposing effects of insulin and amino-acid deficiency on protein translation. Biochem J 344: 427-431.

Naya, F.J., Mercer, B., Shelton, J., Richardson, J.A., Williams, R.S., and Olson, E.N. 2000. Stimulation of Slow Skeletal Muscle Fiber Gene Expression by Calcineurin in Vivo 10.1074/jbc.275.7.4545. J. Biol. Chem. 275: 4545-4548.

Nelson, A.G., Arnall, D.A., Loy, S.F., Silvester, L.J., and Conlee, R.K. 1990. Consequences of combining strength and endurance training regimens. Phys Ther 70: 287-297.

Neufer, P.D., and Dohm, G.L. 1993. Exercise induces a transient increase in transcription of the GLUT-4 gene in skeletal muscle. Am J Physiol Cell Physiol 265: C1597-1603.

Nielsen, J.N., Frøsig, C., Sajan, M.P., Miura, A., Standaert, M.L., Graham, D.A., Wojtaszewski, J.F.P., Farese, R.V., and Richter, E.A. 2003a. Increased atypical PKC activity in endurance-trained human skeletal muscle. Biochem Biophys Res Commun 312: 1147-1153.

Nielsen, J.N., Mustard, K.J.W., Graham, D.A., Yu, H., MacDonald, C.S., Pilegaard, H., Goodyear, L.J., Hardie, D.G., Richter, E.A., and Wojtaszewski, J.F.P. 2003b. 5'-AMPactivated protein kinase activity and subunit expression in exercise-trained human skeletal muscle. J Appl Physiol 94: 631-641.

Norrbom, J., Sundberg, C.J., Ameln, H., Kraus, W.E., Jansson, E., and Gustafsson, T. 2003. PGC-1 $\alpha$ mRNA expression is influenced by metabolic perturbation in exercising human skeletal muscle. J Appl Physiol 96: 189-194. 
Oberkofler, H., Esterbauer, H., Linnemayr, V., Strosberg, A.D., Krempler, F., and Patsch, W. 2002. Peroxisome Proliferator-activated Receptor PPAR $\gamma$ Coactivator-1 Recruitment Regulates PPAR Subtype Specificity. J. Biol. Chem. 277: 16750-16757.

Ohanna, M., Sobering, A.K., Lapointe, T., Lorenzo, L., Praud, C., Petroulakis, E., Sonenberg, N., Kelly, P.A., Sotiropoulos, A., and Pende, M. 2005. Atrophy of S6K1-/- skeletal muscle cells reveals distinct mTOR effectors for cell cycle and size control. Nat Cell Biol. 7: 286-294.

Olson, E.N., and Williams, R.S. 2000. Calcineurin Signaling and Muscle Remodeling. Cell 101: 689-692.

Ostrowski, K., Rohde, T., Asp, S., Schjerling, P., and Pedersen, B.K. 1999. Pro- and antiinflammatory cytokine balance in strenuous exercise in humans. $J$ Physiol 515: 287291.

Park, I.-H., Erbay, E., Nuzzi, P., and Chen, J. 2005. Skeletal myocyte hypertrophy requires mTOR kinase activity and S6K1. Experimental Cell Research 309: 211-219.

Parkington, J.D., LeBrasseur, N.K., Siebert, A.P., and Fielding, R.A. 2004. Contractionmediated mTOR, p70S6k, and ERK1/2 phosphorylation in aged skeletal muscle. $J$ Appl Physiol 97: 243-248.

Parkington, J.D., Siebert, A.P., LeBrasseur, N.K., and Fielding, R.A. 2003. Differential activation of mTOR signaling by contractile activity in skeletal muscle. Am J Physiol Regul Integr Comp Physiol 285: R1086-1090.

Parsons, S.A., Millay, D.P., Wilkins, B.J., Bueno, O.F., Tsika, G.L., Neilson, J.R., Liberatore, C.M., Yutzey, K.E., Crabtree, G.R., Tsika, R.W., et al. 2004. Genetic Loss of Calcineurin Blocks Mechanical Overload-induced Skeletal Muscle Fiber Type Switching but Not Hypertrophy 10.1074/jbc.M313800200. J. Biol. Chem. 279: $26192-$ 26200.

Pavitt, G.D. 2005. eIF2B, a mediator of general and gene-specific translational control. Biochem Soc Trans 33: 1487-1492.

Perkins, N.D., and Gilmore, T.D. 2006. Good cop, bad cop: the different faces of NF-кB. Cell Death Differ 13: 759-772.

Petersen, A.M.W., and Pedersen, B.K. 2005. The anti-inflammatory effect of exercise. J Appl Physiol 98: 1154-1162.

Petrella, J.K., Kim, J.-s., Cross, J.M., Kosek, D.J., and Bamman, M.M. 2006. Efficacy of myonuclear addition may explain differential myofiber growth among resistance trained young and older men and women. Am J Physiol Endocrinol Metab Epub ahead of print: 00190.02006.

Phillips, S.M., Tipton, K.D., Aarsland, A., Wolf, S.E., and Wolfe, R.R. 1997. Mixed muscle protein synthesis and breakdown after resistance exercise in humans. Am J Physiol Endocrinol Metab 36: E99-E107.

Pilegaard, H., Ordway, G.A., Saltin, B., and Neufer, P.D. 2000. Transcriptional regulation of gene expression in human skeletal muscle during recovery from exercise. Am $J$ Physiol Endocrinol Metab 279: E806-E814.

Pilegaard, H., Osada, T., Andersen, L.T., Helge, J.W., Saltin, B., and Neufer, P.D. 2005. Substrate availability and transcriptional regulation of metabolic genes in human skeletal muscle during recovery from exercise. Metabolism 54: 1048-1055.

Plomgaard, P., Bouzakri, K., Krogh-Madsen, R., Mittendorfer, B., Zierath, J.R., and Pedersen, B.K. 2005. Tumor Necrosis Factor- $\alpha$ Induces Skeletal Muscle Insulin Resistance in Healthy Human Subjects via Inhibition of Akt Substrate 160 Phosphorylation. Diabetes 54: 2939-2945.

Psilander, N., Damsgaard, R., and Pilegaard, H. 2003. Resistance exercise alters MRF and IGF-1 mRNA content in human skeletal muscle. J Appl Physiol 95: 1038-1044. 
Pullen, N., Dennis, P.B., Andjelkovic, M., Dufner, A., Kozma, S.C., Hemmings, B.A., and Thomas, G. 1998. Phosphorylation and Activation of p70s6k by PDK1. Science 279: 707-710.

Putman, C., Xu, X., Gillies, E., MacLean, I., and Bell, G. 2004. Effects of strength, endurance and combined training on myosin heavy chain content and fibre-type distribution in humans. Eur J Appl Physiol 92: 376 - 384.

Rasmussen, B.B., and Winder, W.W. 1997. Effect of exercise intensity on skeletal muscle malonyl-CoA and acetyl-CoA carboxylase. J Appl Physiol 83: 1104-1109.

Rauch, C., and Loughna, P.T. 2005. Static stretch promotes MEF2A nuclear translocation and expression of neonatal myosin heavy chain in $\mathrm{C} 2 \mathrm{C} 12$ myocytes in a calcineurin- and p38-dependent manner 10.1152/ajpcell.00346.2004. Am J Physiol Cell Physiol 288: C593-605.

Raue, U., Slivka, D., Jemiolo, B., Hollon, C., and Trappe, S. 2006. Myogenic gene expression at rest and after a bout of resistance exercise in young (18-30 yr) and old (80-89 yr) women. J Appl Physiol 101: 53-59.

Raught, B., Peiretti, F., Gingras, A., Livingstone, M., Shahbazian, D., Mayeur, G., Polakiewicz, R., Sonenberg, N., and Hershey, J. 2004. Phosphorylation of eucaryotic translation initiation factor 4B Ser422 is modulated by S6 kinases. EMBO J 23: 17611769.

Reid, M.B. 2005. Response of the ubiquitin-proteasome pathway to changes in muscle activity. Am J Physiol Regul Integr Comp Physiol 288: R1423-1431.

Reisz-Porszasz, S., Bhasin, S., Artaza, J.N., Shen, R., Sinha-Hikim, I., Hogue, A., Fielder, T.J., and Gonzalez-Cadavid, N.F. 2003. Lower skeletal muscle mass in male transgenic mice with muscle-specific over expression of myostatin. Am J Physiol Endocrinol Metab 285: E876-888.

Rena, G., Woods, Y.L., Prescott, A.R., Peggie, M., Unterman, T.G., Williams, M.R., and Cohen, P. 2002. Two novel phosphorylation sites on FKHR that are critical for its nuclear exclusion. EMBO J. 21: 2263-2271.

Rennie, M.J., and Tipton, K.D. 2000. Protein and amino acid metabolism during and after exercise and the effects of nutrition. Annu Rev Nutr 20: 457-483.

Rennie, M.J., Wackerhage, H., Spangenburg, E.E., and Booth, F.W. 2004. Control of the size of the human muscle mass. Annu Rev Physiol 66: 799-828.

Reynolds, I., Thomas H., Reid, P., Larkin, L.M., and Dengel, D.R. 2004. Effects of aerobic exercise training on the protein kinase $\mathrm{B}(\mathrm{PKB}) /$ mammalian target of rapamycin (mTOR) signaling pathway in aged skeletal muscle. Experimental Gerontology 39: 379-385.

Reynolds, T.H., IV, Bodine, S.C., and Lawrence, J.C., Jr. 2002. Control of Ser2448 Phosphorylation in the Mammalian Target of Rapamycin by Insulin and Skeletal Muscle Load. J. Biol. Chem. 277: 17657-17662.

Richter, J.D., and Sonenberg, N. 2005. Regulation of cap-dependent translation by eIF4E inhibitory proteins. 433: 477-480.

Riedy, M., Moore, R.L., and Gollnick, P.D. 1985. Adaptive response of hypertrophied skeletal muscle to endurance training. J Appl Physiol 59: 127-131.

Ririe, K.M., Rasmussen, R.P., and Wittwer, C.T. 1997. Product Differentiation by Analysis of DNA Melting Curves during the Polymerase Chain Reaction. Anal Biochem 245: 154160.

Rommel, C., Bodine, S.C., Clarke, B.A., Rossman, R., Nunez, L., Stitt, T.N., Yancopoulos, G.D., and Glass, D.J. 2001. Mediation of IGF-1-induced skeletal myotube hypertrophy by PI(3)K/Akt/mTOR and PI(3)K/Akt/GSK3 pathways. Nat Cell Biol 3: 1009-1013. 
Rommel, C., Clarke, B.A., Zimmermann, S., Nuñez, L., Rossman, R., Reid, K., Moelling, K., Yancopoulos, G.D., and Glass, D.J. 1999. Differientiation stage-specific inhibition of the Raf-MEK-ERK pathway by Akt. Science 286: 1738-1741.

Rose, A.J., Broholm, C., Kiillerich, K., Finn, S.G., Proud, C.G., Rider, M.H., Richter, E.A., and Kiens, B. 2005. Exercise rapidly increases eukaryotic elongation factor 2 phosphorylation in skeletal muscle of men 10.1113/jphysiol.2005.097154. J Physiol (Lond) 569: 223-228.

Rose, A.J., and Hargreaves, M. 2003. Exercise increases Ca2+-calmodulin-dependent protein kinase II activity in human skeletal muscle 10.1113/jphysiol.2003.054171. J Physiol (Lond) 553: 303-309.

Rose, A.J., Kiens, B., and Richter, E.A. 2006. Ca2+-calmodulin dependent protein kinase expression and signalling in skeletal muscle during exercise 10.1113/jphysiol.2006.111757. J Physiol (Lond): jphysiol.2006.111757.

Roth, S.M., Martel, G.F., Ferrell, R.E., Metter, E.J., Hurley, B.F., and Rogers, M.A. 2003. Myostatin gene expression is reduced in humans with heavy-resistance strength training. Exp Biol Med 228: 706-709.

Roux, P.P., and Blenis, J. 2004. ERK and p38 MAPK-activated protein kinases: a family of protein kinases with diverse biological functions. Microbiol Mol Biol Rev 68: 320344.

Russell, A.P., Hesselink, M.K.C., Lo, S.K., and Schrauwen, P. 2005. Regulation of metabolic transcriptional co-activators and transcription factors with acute exercise. FASEB J.: 04-3168fje.

Ruvinsky, I., and Meyuhas, O. 2006. Ribosomal protein S6 phosphorylation: from protein synthesis to cell size. Trends Biochem Sci 31: 342-348.

Ryazanov, A.G. 1987. Ca2+/calmodulin-dependent phosphorylation of elongation factor 2 . FEBS Letters 214: 331-334.

Sacheck, J.M., Ohtsuka, A., McLary, S.C., and Goldberg, A.L. 2004. IGF-I stimulates muscle growth by suppressing protein breakdown and expression of atrophy-related ubiquitin ligases, atrogin-1 and MuRF1. Am J Physiol Endocrinol Metab 287: E591-E601.

Sakamoto, K., Arnolds, D.E.W., Ekberg, I., Thorell, A., and Goodyear, L.J. 2004a. Exercise regulates Akt and glycogen synthase kinase-3 activities in human skeletal muscle. Biochem Biophys Res Commun 319: 419-425.

Sakamoto, K., Aschenbach, W.G., Hirshman, M.F., and Goodyear, L.J. 2003. Akt signaling in skeletal muscle: regulation by exercise and passive stretch. Am J Physiol Endocrinol Metab 285: E1081-1088.

Sakamoto, K., and Goodyear, L.J. 2002. Exercise Effects on Muscle Insulin Signaling and Action: Invited Review: Intracellular signaling in contracting skeletal muscle. $J$ Appl Physiol 93: 369-383.

Sakamoto, K., Goransson, O., Hardie, D.G., and Alessi, D.R. 2004b. Activity of LKB1 and AMPK-related kinases in skeletal muscle: effects of contraction, phenformin, and AICAR 10.1152/ajpendo.00074.2004. Am J Physiol Endocrinol Metab 287: E310317.

Sakamoto, K., Hirshman, M.F., Aschenbach, W.G., and Goodyear, L.J. 2002. Contraction Regulation of Akt in Rat Skeletal Muscle. J. Biol. Chem. 277: 11910-11917.

Sakamoto, K., McCarthy, A., Smith, D., Green, K.A., Hardie, D.G., Ashworth, A., and Alessi, A.R. 2005. Deficiency of LKB1 in skeletal muscle prevents AMPK activation and glucose uptake during contraction. EMBO J 24: 1810-1820.

Sakuma, K., Nishikawa, J., Nakao, R., Watanabe, K., Totsuka, T., Nakano, H., Sano, M., and Yasuhara, M. 2003. Calcineurin is a potent regulator for skeletal muscle regeneration by association with NFATc1 and GATA-2. Acta Neuropathol 105: 271-280.

Saltin, B. 1969. Oxygen uptake and cardiac output during maximal treadmill and bicycle exercise. Mal Cardiovasc 10: 393-399. 
Sanders Williams, R., and Neufer, P.D. 1996. Regulation of gene expression in skeletal muscle by contractile activity. In Handbook of Physiology. Section 12: Exercise: Regulation and Intergration of Multiple Systems. (eds. L.B. Rowell, and J.T. Shepherd). Oxford University Press, New York.

Sandri, M., Podhorska-Okolow, M., Geromel, V., Rizzi, C., Arslan, P., Franceschi, C., and Carraro, U. 1997. Exercise induces myonuclear ubiquitination and apoptosis in dystrophin-deficient muscle of mice. J Neuropathol Exp Neurol 56: 45-57.

Sandri, M., Sandri, C., Gilbert, A., Skurk, C., Calabria, E., Picard, A., Walsh, K., Schiaffino, S., Lecker, S.H., and Goldberg, A.L. 2004. Foxo Transcription Factors Induce the Atrophy-Related Ubiquitin Ligase Atrogin-1 and Cause Skeletal Muscle Atrophy. Cell 117: 399-412.

Santel, A., Frank, S., Gaume, B., Herrler, M., Youle, R.J., and Fuller, M.T. 2003. Mitofusin-1 protein is a generally expressed mediator of mitochondrial fusion in mammalian cells. J Cell Sci 116: 2763-2774.

Santel, A., and Fuller, M. 2001. Control of mitochondrial morphology by a human mitofusin. $J$ Cell Sci 114: 867-874.

Sarbassov, D.D., Ali, S.M., Kim, D.-H., Guertin, D.A., Latek, R.R., Erdjument-Bromage, H., Tempst, P., and Sabatini, D.M. 2004. Rictor, a Novel Binding Partner of mTOR, Defines a Rapamycin-Insensitive and Raptor-Independent Pathway that Regulates the Cytoskeleton. Current Biology 14: 1296-1302.

Sarbassov, D.D., Ali, S.M., and Sabatini, D.M. 2005a. Growing roles for the mTOR pathway. Current Opinion in Cell Biology. 17: 596-603.

Sarbassov, D.D., Ali, S.M., Sengupta, S., Sheen, J.-H., Hsu, P.P., Bagley, A.F., Markhard, A.L., and Sabatini, D.M. 2006. Prolonged Rapamycin Treatment Inhibits mTORC2 Assembly and Akt/PKB. Molecular Cell 22: 159-168.

Sarbassov, D.D., Guertin, D.A., Ali, S.M., and Sabatini, D.M. 2005b. Phosphorylation and Regulation of Akt/PKB by the Rictor-mTOR Complex. Science 307: 1098-1101.

Sartorelli, V., and Fulco, M. 2004. Molecular and Cellular Determinants of Skeletal Muscle Atrophy and Hypertrophy. Sci STKE 2004: re11-.

Scarpulla, R.C. 2002. Transcriptional activators and coactivators in the nuclear control of mitochondrial function in mammalian cells. Gene 286: 81-89.

Scarpulla, R.C. 2006. Nuclear control of respiratory gene expression in mammalian cells. $J$ Cell Biochem 97: 673-683.

Scherer, D., Brockman, J., Chen, Z., Maniatis, T., and Ballard, D. 1995. Signal-Induced Degradation of IкB $\alpha$ Requires Site-Specific Ubiquitination. PNAS 92: 11259-11263.

Schertzer, J.D., Green, H.J., Duhamel, T.A., and Tupling, A.R. 2003. Mechanisms underlying increases in SR Ca2+-ATPase activity after exercise in rat skeletal muscle 10.1152/ajpendo.00190.2002. Am J Physiol Endocrinol Metab 284: E597-610.

Schertzer, J.D., Green, H.J., Fowles, J.R., Duhamel, T.A., and Tupling, A.R. 2004. Effects of prolonged exercise and recovery on sarcoplasmic reticulum $\mathrm{Ca} 2+$ cycling properties in rat muscle homogenates. Acta Physiol Scand 180: 195-208.

Schieke, S.M., Phillips, D., McCoy, J.P., Jr, Aponte, A.M., Shen, R.-F., Balaban, R.S., and Finkel, T. 2006. The mTOR pathway regulates mitochondrial oxygen consumption and oxidative capacity. J. Biol. Chem. Epub ahead of print.

Schuelke, M., Wagner, K.R., Stolz, L.E., Hubner, C., Riebel, T., Komen, W., Braun, T., Tobin, J.F., and Lee, S.-J. 2004. Myostatin mutation associated with gross hypertrophy in a child. $N$ Engl J Med 350: 2682-2688.

Schwabe, R.F., and Sakurai, H. 2005. IKK $\beta$ phosphorylates p65 at S468 in transactivaton domain 2. FASEB J.: 05-3736fje.

Scicchitano, B.M., Spath, L., Musaro, A., Molinaro, M., Rosenthal, N., Nervi, C., and Adamo, S. 2005. Vasopressin-dependent Myogenic Cell Differentiation Is Mediated 
by Both $\mathrm{Ca} 2+/$ Calmodulin-dependent Kinase and Calcineurin Pathways 10.1091/mbc.E05-01-0055. Mol. Biol. Cell 16: 3632-3641.

Scott, P.H., Brunn, G.J., Kohn, A.D., Roth, R.A., and Lawrence, J.C., Jr. 1998. Evidence of insulin-stimulated phosphorylation and activation of the mammalian target of rapamycin mediated by a protein kinase B signaling pathway. PNAS 95: 7772-7777.

Shahbazian, D., Roux, P.P., Mieulet, V., Cohen, M.S., Raught, B., Taunton, J., Hershey, J.W., Blenis, J., Pende, M., and Sonenberg, N. 2006. The mTOR/PI3K and MAPK pathways converge on eIF4B to control its phosphorylation and activity. EMBO $J$ 25: 2781-2791.

Shen, W.-H., Boyle, D.W., Wisniowski, P., Bade, A., and Liechty, E.A. 2005. Insulin and IGF-I stimulate the formation of the eukaryotic initiation factor $4 \mathrm{~F}$ complex and protein synthesis in $\mathrm{C} 2 \mathrm{C} 12$ myotubes independent of availability of external amino acids. J Endocrinol 185: 275-289.

Sherwood, D.J., Dufresne, S.D., Markuns, J.F., Cheatham, B., Moller, D.E., Aronson, D., and Goodyear, L.J. 1999. Differential regulation of MAP kinase, p70S6K, and Akt by contraction and insulin in rat skeletal muscle. Am J Physiol Endocrinol Metab 276: E870-878.

Shima, H., Pende, M., Chen, Y., Fumagalli, S., Thomas, G., and Kozma, S. 1998. Disruption of the $\mathrm{p} 70(\mathrm{~s} 6 \mathrm{k}) / \mathrm{p} 85(\mathrm{~s} 6 \mathrm{k})$ gene reveals a small mouse phenotype and a new functional S6 kinase. EMBO J 17: 6649-6659.

Short, K.R., Vittone, J.L., Bigelow, M.L., Proctor, D.N., Rizza, R.A., Coenen-Schimke, J.M., and Nair, K.S. 2003. Impact of aerobic exercise training on age-related changes in insulin sensitivity and muscle oxidative capacity. Diabetes 52: 1888-1896.

Simone, C., Forcales, C.S., Hill, D.A., Imbalzano, A.N., Latella, L., and Puri, P.L. 2004. p38 pathway targets SWI-SNF chromatin-remodeling complex to muscle-specific loci. Nat Genet 36: 738-742.

Siu, P.M., Donley, D.A., Bryner, R.W., and Alway, S.E. 2004. Myogenin and oxidative enzyme gene expression levels are elevated in rat soleus muscles after endurance training. J Appl Physiol Epub ahead of print: 00534.02003.

Sleeman, M., Wortley, K., Lai, K., Gowen, L., Kintner, J., Kline, W., Garcia, K., Stitt, T., Yancopoulos, G., Wiegand, S., et al. 2005. Absence of the lipid phosphatase SHIP2 confers resistance to dietary obesity. Nat Med 11: 199-205.

Smith, M.A., and Reid, M.B. 2006. Redox modulation of contractile function in respiratory and limb skeletal muscle. Respiratory Physiology \& Neurobiology In Press, Corrected Proof.

Song, Y.H., Godard, M., Li, Y., Richmond, S.R., Rosenthal, N., and Delafontaine, P. 2005. Insulin-Like Growth Factor I-Mediated Skeletal Muscle Hypertrophy Is Characterized by Increased mTOR-p70S6K Signaling without Increased Akt Phosphorylation. $J$ Investig Med 53: 135-142.

Soriano, F.X., Liesa, M., Bach, D., Chan, D.C., Palacin, M., and Zorzano, A. 2006. Evidence for a Mitochondrial Regulatory Pathway Defined by Peroxisome ProliferatorActivated Receptor- $\gamma$ Coactivator-1 $\alpha$, Estrogen-Related Receptor- $\alpha$, and Mitofusin 2. Diabetes 55: 1783-1791.

Southgate, R.J., Bruce, C.R., Carey, A.L., Steinberg, G.R., Walder, K., Monks, R., Watt, M.J., Hawley, J.A., Birnbaum, M.J., and Febbraio, M.A. 2005. PGC-1\&alpha; gene expression is down-regulated by Akt-mediated phosphorylation and nuclear exclusion of FoxO1 in insulin-stimulated skeletal muscle. FASEB J. 19: 2072-2074.

Spangenburg, E.E., Abraha, T., Childs, T.E., Pattison, J.S., and Booth, F.W. 2002. Skeletal muscle IGF-binding protein-3 and -5 expressions are age, muscle and load dependent. Am J Physiol Endocrinol Metab 284: E340-E350. 
Spangenburg, E.E., and McBride, T.A. 2006. Inhibition of stretch-activated channels during eccentric muscle contraction attenuates p70S6K activation 10.1152/japplphysiol.00619.2005. J Appl Physiol 100: 129-135.

Spriet, L.L. 2002. Regulation of skeletal muscle fat oxidation during exercise in humans. Med Sci Sports Exerc 34: 1477-1484.

Sriwijitkamol, A., Christ-Roberts, C., Berria, R., Eagan, P., Pratipanawatr, T., DeFronzo, R.A., Mandarino, L.J., and Musi, N. 2006. Reduced Skeletal Muscle Inhibitor of $\kappa B \beta$ Content Is Associated With Insulin Resistance in Subjects With Type 2 Diabetes: Reversal by Exercise Training. Diabetes 55: 760-767.

Sriwijitkamol, A., Ivy, J.L., Christ-Roberts, C., DeFronzo, R.A., Mandarino, L.J., and Musi, N. 2005. LKB1-AMPK Signaling in Muscle from Obese Insulin Resistant Zucker Rats and Effects of Training 10.1152/ajpendo.00429.2005. Am J Physiol Endocrinol Metab: 00429.02005.

Steinbrecher, K.A., Wilson, W., III, Cogswell, P.C., and Baldwin, A.S. 2005. Glycogen Synthase Kinase $3 \beta$ Functions To Specify Gene-Specific, NF- $\kappa B-D e p e n d e n t$ Transcription. Mol. Cell. Biol. 25: 8444-8455.

Stephens, L., Anderson, K., Stokoe, D., Erdjument-Bromage, H., Painter, G.F., Holmes, A.B., Gaffney, P.R.n.J., Reese, C.B., McCormick, F., Tempst, P., et al. 1998. Protein Kinase B Kinases That Mediate Phosphatidylinositol 3,4,5-Trisphosphate-Dependent Activation of Protein Kinase B 10.1126/science.279.5351.710. Science 279: 710-714.

Stephens, T.J., Chen, Z.-P., Canny, B.J., Michell, B.J., Kemp, B.E., and McConell, G.K. 2002. Progressive increase in human skeletal muscle AMPK $\alpha 2$ activity and ACC phosphorylation during exercise 10.1152/ajpendo.00101.2001. Am $J$ Physiol Endocrinol Metab 282: E688-694.

Stitt, T.N., Drujan, D., Clarke, B.A., Panaro, F., Timofeyva, Y., Kline, W.O., Gonzalez, M., Yancopoulos, G.D., and Glass, D.J. 2004. The IGF/PI3K/Akt pathway prevents expression of muscle atrophy-induced ubiquitin ligases by inhibiting FOXO transcription factors. Mol Cell 14: 395-403.

Stofan, D.A., Callahan, L.A., DiMarco, A.F., Nethery, D.E., and Supinski, G.S. 2000. Modulation of Release of Reactive Oxygen Species by the Contracting Diaphragm. Am. J. Respir. Crit. Care Med. 161: 891-898.

Stone, J., Brannon, T., Haddad, F., Qin, A., and Baldwin, K.M. 1996. Adaptive responses of hypertrophying skeletal muscle to endurance training. J Appl Physiol 81: 665-672.

Stupka, N., Tarnopolsky, M.A., Yardley, N.J., and Phillips, S.M. 2001. Cellular adaptation to repeated eccentric exercise-induced muscle damage. J Appl Physiol 91: 1669-1678.

Suelves, M., Lluis, F., Ruiz, V., Nebreda, A.R., and Munoz-Canoves, P. 2004. Phosphorylation of MRF4 transactivation domain by $\mathrm{p} 38$ mediates repression of specific myogenic genes. EMBO J 23: 365-375.

Sugden, M.C., and Holness, M.J. 2003. Recent advances in mechanisms regulating glucose oxidation at the level of the pyruvate dehydrogenase complex by PDKs. Am J Physiol Endocrinol Metab 284: E855-862.

Takano, A., Usui, I., Haruta, T., Kawahara, J., Uno, T., Iwata, M., and Kobayashi, M. 2001. Mammalian Target of Rapamycin Pathway Regulates Insulin Signaling via Subcellular Redistribution of Insulin Receptor Substrate 1 and Integrates Nutritional Signals and Metabolic Signals of Insulin. Mol. Cell. Biol. 21: 5050-5062.

Talmadge, R., Otis, J., Rittler, M., Garcia, N., Spencer, S., Lees, S., and Naya, F. 2004. Calcineurin activation influences muscle phenotype in a muscle-specific fashion. $B M C$ Cell Biol 5:28.

Tang, X., Powelka, A.M., Soriano, N.A., Czech, M.P., and Guilherme, A. 2005. PTEN, but Not SHIP2, Suppresses Insulin Signaling through the Phosphatidylinositol 3Kinase/Akt Pathway in 3T3-L1 Adipocytes 10.1074/jbc.M501949200. J. Biol. Chem. 280: $22523-22529$. 
Taniguchi, C., Emanuelli, B., and Kahn, C. 2006. Critical nodes in signalling pathways: insights into insulin action. Nat Rev Mol Cell Biol 7: 85-96.

Taylor, E.B., Lamb, J.D., Hurst, R.W., Chesser, D.G., Ellingson, W.J., Greenwood, L.J., Porter, B.B., Herway, S.T., and Winder, W.W. 2005. Endurance training increases skeletal muscle LKB1 and PGC-1 $\alpha$ protein abundance: effects of time and intensity 10.1152/ajpendo.00237.2005. Am J Physiol Endocrinol Metab 289: E960-968.

Tee, A.R., Fingar, D.C., Manning, B.D., Kwiatkowski, D.J., Cantley, L.C., and Blenis, J. 2002. Tuberous sclerosis complex-1 and -2 gene products function together to inhibit mammalian target of rapamycin (mTOR)-mediated downstream signaling. PNAS 99: 13571-13576.

Terada, S., Goto, M., Kato, M., Kawanaka, K., Shimokawa, T., and Tabata, I. 2002. Effects of low-intensity prolonged exercise on PGC-1 mRNA expression in rat epitrochlearis muscle. Biochemical and Biophysical Research Communications 296: 350-354.

Terada, S., Kawanaka, K., Goto, M., Shimokawa, T., and Tabata, I. 2005. Effects of highintensity intermittent swimming on PGC-1 $\alpha$ protein expression in rat skeletal muscle. Acta Physiol Scand 184: 59-65.

Terada, S., and Tabata, I. 2003. Effects of acute bouts of running and swimming exercise on PGC-1 $\alpha$ protein expression in rat epitrochlearis and soleus muscle. Am J Physiol Endocrinol Metab 286: E208-216.

Teran-Garcia, M., Rankinen, T., Koza, R.A., Rao, D.C., and Bouchard, C. 2005. Endurance training-induced changes in insulin sensitivity and gene expression. Am J Physiol Endocrinol Metab 288: E1168-1178.

Tergaonkar, V., Correa, R.G., Ikawa, M., and Verma, I.M. 2005. Distinct roles of I[kappa]B proteins in regulating constitutive NF- $\kappa \mathrm{B}$ activity. 7: 921-923.

Thomas, M., Langley, B., Berry, C., Sharma, M., Kirk, S., Bass, J., and Kambadur, R. 2000. Myostatin, a Negative Regulator of Muscle Growth, Functions by Inhibiting Myoblast Proliferation. J. Biol. Chem. 275: 40235-40243.

Thompson, H.S., Maynard, E.B., Morales, E.R., and Scordilis, S.P. 2003. Exercise-induced HSP27, HSP70 and MAPK responses in human skeletal muscle. Acta Physiol Scand 178: $61-72$.

Thomson, D.M., and Gordon, S.E. 2006. Impaired Overload-induced Muscle Growth is associated with Diminished Translational Signaling in Aged Rat Fast-twitch Skeletal Muscle 10.1113/jphysiol.2006.107490. J Physiol (Lond): jphysiol.2006.107490.

Thong, F.S.L., Derave, W., UrsØ, B., Kiens, B., and Richter, E.A. 2003. Prior exercise increases basal and insulin-induced p38 mitogen activated protein kinase phosphorylation in human skeletal muscle. J Appl Physiol 94: 2337-2341.

Thorell, A., Hirshman, M.F., Nygren, J., Jorfeldt, L., Wojtaszewski, J.F.P., Dufresne, S.D., Horton, E.S., Ljungqvist, O., and Goodyear, L.J. 1999. Exercise and insulin cause GLUT-4 translocation in human skeletal muscle. Am J Physiol Endocrinol Metab 277: E733-741.

Tidball, J.G. 2005. Mechanical signal transduction in skeletal muscle growth and adaptation 10.1152/japplphysiol.01178.2004. J Appl Physiol 98: 1900-1908.

Tintignac, L.A., Lagirand, J., Batonnet, S., Sirri, V., Leibovitch, M.P., and Leibovitch, S.A. 2005. Degradation of MyoD Mediated by the SCF (MAFbx) Ubiquitin Ligase. J. Biol. Chem. 280: 2847-2856.

Tischler, M.E., Rosenberg, S., Satarug, S., Henriksen, E., Kirby, C., Tome, M., and Chase, P. 1990. Different mechanisms of increased proteolysis in atrophy induced by denervation or unweighting of rat soleus muscle. Metabolism 39: 756-763.

Toyoda, T., Tanaka, S., Ebihara, K., Masuzaki, H., Hosoda, K., Sato, K., Fushiki, T., Nakao, K., and Hayashi, T. 2006. Low-intensity contraction activates the $\alpha 1$-isoform of 5'AMP-activated protein kinase in rat skeletal muscle 10.1152/ajpendo.00395.2005. Am J Physiol Endocrinol Metab 290: E583-590. 
Tseng, B.S., Kasper, C.E., and Edgerton, V.R. 1994. Cytoplasm-to-nucleus ratios and succinate dehydrogenase activities in adult rat slow and fast muscle fibers. Cell Tissue Res 275: 39-49.

Tseng, B.S., Marsh, D.R., Hamilton, M.T., and Booth, F.W. 1995. Strength and aerobic training attenuate muscle wasting and improve resistance to the development of disability with aging. J Gerontol A Biol Sci Med Sci 50A: 113-119.

Tunstall, R.J., Mehan, K.A., Wadley, G.D., Collier, G.R., Bonen, A., Hargreaves, M., and Cameron-Smith, D. 2002. Exercise training increases lipid metabolism gene expression in human skeletal muscle. Am J Physiol Endocrinol Metab 283: E66-E72.

Turinsky, J., and Damrau-Abney, A. 1999. Akt kinases and 2-deoxyglucose uptake in rat skeletal muscles in vivo: study with insulin and exercise. Am J Physiol Regul Integr Comp Physiol 276: R277-282.

Vandenburgh, H.H., Karlisch, P., Shansky, J., and Feldstein, R. 1991. Insulin and IGF-I induce pronounced hypertrophy of skeletal myofibers in tissue culture. Am J Physiol Cell Physiol 260: C475-484.

Vary, T.C. 2006. IGF-I stimulates protein synthesis in skeletal muscle through multiple signaling pathways during sepsis. Am J Physiol Regul Integr Comp Physiol 290: R313-321.

Vary, T.C., Deiter, G., and Kimball, S.R. 2002. Phosphorylation of eukaryotic initiation factor eIF2B $\varepsilon$ in skeletal muscle during sepsis 10.1152/ajpendo.00171.2002. Am J Physiol Endocrinol Metab 283: E1032-1039.

Vary, T.C., and Lynch, C.J. 2006. Meal feeding enhances formation of eIF4F in skeletal muscle: role of increased eIF4E availability and eIF4G phosphorylation 10.1152/ajpendo.00460.2005. Am J Physiol Endocrinol Metab 290: E631-642.

Vashisht Gopal, Y., Arora, T., and Van Dyke, M. 2006. Tumor necrosis factor-alpha depletes histone deacetylase 1 protein through IKK2. EMBO Rep 7: 291-296.

Vega, R.B., Huss, J.M., and Kelly, D.P. 2000. The Coactivator PGC-1 Cooperates with Peroxisome Proliferator-Activated Receptor $\alpha$ in Transcriptional Control of Nuclear Genes Encoding Mitochondrial Fatty Acid Oxidation Enzymes. Mol. Cell. Biol. 20: $1868-1876$.

Vissing, K., Andersen, J.L., Harridge, S.D.R., Sandri, C., Hartkopp, A., Kjaer, M., and Schjerling, P. 2005. Gene expression of myogenic factors and phenotype-specific markers in electrically stimulated muscle of paraplegics. J Appl Physiol 99: 164-172.

Vissing, K., Andersen, J.L., and Schjerling, P. 2004. Are exercise-induced genes induced by exercise? FASEB J.: 04-2084fje.

Vyas, D.R., Spangenburg, E.E., Abraha, T.W., Childs, T.E., and Booth, F.W. 2002. GSK-3 $\beta$ negatively regulates skeletal myotube hypertrophy. Am J Physiol Cell Physiol 283: C545-551.

Wadley, G.D., Lee-Young, R.S., Canny, B.J., Wasuntarawat, C., Chen, Z.P., Hargreaves, M., Kemp, B.E., and McConell, G.K. 2006. Effect of exercise intensity and hypoxia on skeletal muscle AMPK signaling and substrate metabolism in humans. Am J Physiol Endocrinol Metab 290: E694-702.

Wadley, G.D., Tunstall, R.J., Sanigorski, A., Collier, G.R., Hargreaves, M., and CameronSmith, D. 2001. Differential effects of exercise on insulin-signaling gene expression in human skeletal muscle. J Appl Physiol 90: 436-440.

Wagner, K.R., Liu, X., Chang, X., and Allen, R.E. 2005. Muscle regeneration in the prolonged absence of myostatin. PNAS 102: 2519-2524.

Wang, X., Beugnet, A., Murakami, M., Yamanaka, S., and Proud, C.G. 2005. Distinct Signaling Events Downstream of mTOR Cooperate To Mediate the Effects of Amino Acids and Insulin on Initiation Factor 4E-Binding Proteins. Mol. Cell. Biol. 25: 25582572. 
Wang, X., Li, W., Williams, M., Terada, N., Alessi, D., and Proud, C. 2001. Regulation of elongation factor 2 kinase by p90(RSK1) and p70 S6 kinase. EMBO J 20: 4370-4379.

Wang, Y., Zhang, C., Yu, R.T., Cho, H.K., Nelson, M.C., Bayuga-Ocampo, C.R., Ham, J., Kang, H., and Evans, R.M. 2004. Regulation of muscle fiber type and running endurance by PPAR $\delta$. PLoS Biol 2: e294.

Welsh, G.I., Stokes, C.M., Wang, X., Sakaue, H., Ogawa, W., Kasuga, M., and Proud, C.G. 1997. Activation of translation initiation factor eIF2B by insulin requires phosphatidyl inositol 3-kinase. FEBS Letters 410: 418-422.

Wick, M.J., Dong, L.Q., Riojas, R.A., Ramos, F.J., and Liu, F. 2000. Mechanism of Phosphorylation of Protein Kinase B/Akt by a Constitutively Active 3Phosphoinositide-dependent Protein Kinase-1 10.1074/jbc.M003937200. J. Biol. Chem. 275: 40400-40406.

Widegren, U., Jiang, X.J., Krook, A., Chibalin, A.V., Björnholm, M., Tally, M., Roth, R.A., Henriksson, J., Wallberg-Henriksson, H., and Zierath, J.R. 1998. Divergent effects of exercise on metabolic and mitogenic signaling pathways in human skeletal muscle. FASEB J. 12: 1379-1389.

Widegren, U., Ryder, J.W., and Zierath, J.R. 2001. Mitogen-activated protein kinase signal transduction in skeletal muscle: effects of exercise and muscle contraction. Acta Physiol Scand 172: 227-238.

Williams, R.S., and Neufer, P.D. 1996. Regulation of gene expression in skeletal muscle by contractile activity. In Handbook of Physiology. Section 12: Exercise: Regulation and Intergration of Multiple Systems. (eds. L.B. Rowell, and J.T. Shepherd), pp. 11241150. Oxford University Press, New York.

Williamson, D., Gallagher, P., Harber, M., Hollon, C., and Trappe, S. 2003. Mitogenactivated protein kinase (MAPK) pathway activation: effects of age and acute exercise on human skeletal muscle. $J$ Physiol 547: 977-987.

Williamson, D.L., Bolster, D.R., Kimball, S.R., and Jefferson, L.S. 2006a. Time course changes in signaling pathways and protein synthesis in $\mathrm{C} 2 \mathrm{C} 12$ myotubes following AMPK activation by AICAR. Am J Physiol Endocrinol Metab 291: E80-89.

Williamson, D.L., Gallagher, P.M., Carroll, C.C., Raue, U., and Trappe, S.W. 2001. Reduction in hybrid single muscle fiber proportions with resistance training in humans. J Appl Physiol 91: 1955-1961.

Williamson, D.L., Kimball, S.R., and Jefferson, L.S. 2005. Acute treatment with TNF- $\alpha$ attenuates insulin-stimulated protein synthesis in cultures of $\mathrm{C} 2 \mathrm{C} 12$ myotubes through a MEK1-sensitive mechanism. Am J Physiol Endocrinol Metab 289: E95-104.

Williamson, D.L., Kubica, N., Kimball, S.R., and Jefferson, L.S. 2006b. Exercise-induced alterations in ERK1/2 and mTOR signaling to regulatory mechanisms of mRNA translation in mouse muscle. J Physiol (Lond): jphysiol.2005.103481.

Willoughby, D.S. 2004. Effects of heavy resistance training on myostatin mRNA and protein expression. Med Sci Sports Exerc 36: 574-582.

Willoughby, D.S., and Nelson, M.J. 2002. Myosin heavy-chain mRNA expression after a single session of heavy-resistance exercise. Med Sci Sports Exerc 34: 1262-1269.

Willoughby, D.S., Taylor, M., and Taylor, L. 2003. Glucocorticoid receptor and ubiquitin expression after repeated eccentric exercise. Med Sci Sports Exerc 35: 2023-2031.

Wilson, C., Hargreaves, M., and Howlett, K.F. 2006. Exercise does not alter subcellular localization, but increases phosphorylation of insulin-signaling proteins in human skeletal muscle. Am J Physiol Endocrinol Metab 290: E341-346.

Winder, W.W. 2001. Energy-sensing and signaling by AMP-activated protein kinase in skeletal muscle. J Appl Physiol 91: 1017-1028.

Witters, L.A., Kemp, B.E., and Means, A.R. 2006. Chutes and Ladders: the search for protein kinases that act on AMPK. Trends in Biochemical Sciences CELEBRATING 30 YEARS OF TiBS 31: 13-16. 
Wojtaszewski, J.F.P., Birk, J.B., Frosig, C., Holten, M., Pilegaard, H., and Dela, F. 2005. 5'AMP activated protein kinase expression in human skeletal muscle: effects of strength training and type 2 diabetes. J Physiol (Lond) 564: 563-573.

Wojtaszewski, J.F.P., Higaki, Y., Hirshman, M.F., Michael, M.D., Dufresne, S.D., Kahn, C.R., and Goodyear, L.J. 1999. Exercise modulates postreceptor insulin signaling and glucose transport in muscle-specific insulin receptor knockout mice. J. Clin. Invest. 104: $1257-1264$.

Wojtaszewski, J.F.P., MacDonald, C., Nielsen, J.N., Hellsten, Y., Hardie, D.G., Kemp, B.E., Kiens, B., and Richter, E.A. 2003. Regulation of 5'AMP-activated protein kinase activity and substrate utilization in exercising human skeletal muscle. Am J Physiol Endocrinol Metab 284: E813-822.

Wojtaszewski, J.F.P., Nielsen, P., Hansen, B.F., Richter, E.A., and Kiens, B. 2000. Isoformspecific and exercise intensity-dependent activation of 5'-AMP-activated protein kinase in human skeletal muscle. J Physiol (Lond) 528: 221-226.

Woods, A., Dickerson, K., Heath, R., Hong, S.-P., Momcilovic, M., Johnstone, S.R., Carlson, M., and Carling, D. 2005. Ca2+/calmodulin-dependent protein kinase kinase- $\beta$ acts upstream of AMP-activated protein kinase in mammalian cells. Cell Metabolism 2: 21-33.

Wretman, C., Lionikas, A., Widegren, U., Lännergren, J., Westerblad, H., and Henriksson, J. 2001. Effects of concentric and eccentric contractions on phosphorylation of MAPK $^{\text {erk1/2 }}$ and MAPK ${ }^{\mathrm{p} 38}$ in isolated rat skeletal muscle. $J$ Physiol 535.1: 155-164.

Wu, H., Kanatous, S.B., Thurmond, F.A., Gallardo, T., Isotani, E., Bassel-Duby, R., and Williams, R.S. 2002. Regulation of Mitochondrial Biogenesis in Skeletal Muscle by CaMK 10.1126/science.1071163. Science 296: 349-352.

Wu, H., Naya, F., McKinsey, T., Mercer, B., Shelton, J., Chin, E., Simard, A., Michel, R., Bassel-Duby, R., Olson, E., et al. 2000a. MEF2 responds to multiple calciumregulated signals in the control of skeletal muscle fiber type. EMBO J 19: 1963-1973.

Wu, Z., Puigserver, P., Andersson, U., Zhang, C., Adelmant, G., Mootha, V., Troy, A., Cinti, S., Lowell, B., Scarpulla, R.C., et al. 1999. Mechanisms Controlling Mitochondrial Biogenesis and Respiration through the Thermogenic Coactivator PGC-1. Cell 98: 115-124.

Wu, Z., Woodring, P.J., Bhakta, K.S., Tamura, K., Wen, F., Feramisco, J.R., Karin, M., Wang, J.Y.J., and Puri, P.L. 2000b. p38 and extracellular signal-regulated kinases regulate the myogenic program at multiple steps. Mol Cell Biol 20: 3951-3964.

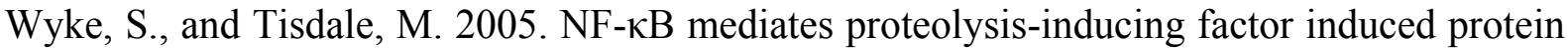
degradation and expression of the ubiquitin-proteasome system in skeletal muscle. $\mathrm{Br}$ J Cancer 92: 711-721.

Xanthoudakis, S., Miao, G., Wang, F., Pan, Y.C., and Curran, T. 1992. Redox activation of Fos-Jun DNA binding activity is mediated by a DNA repair enzyme. $E M B O J$ 11: 3323-3335.

Yang, Y., Creer, A., Jemiolo, B., and Trappe, S. 2005. Time course of myogenic and metabolic gene expression in response to acute exercise in human skeletal muscle. $J$ Appl Physiol 98: 1745-1752.

Yang, Y., Jemiolo, B., and Trappe, S.W. 2006. Proteolytic mRNA Expression in Response to Acute Resistance Exercise in Human Single Skeletal Muscle Fibers. J Appl Physiol Epub ahead of print: 00438.02006 .

Yu, M., Blomstrand, E., Chibalin, A.V., Krook, A., and Zierath, J.R. 2001. Marathon running increases ERK 1/2 and p38 MAP kinase signalling to downstream targets in human skeletal muscle. J Physiol 536: 273-282.

Yu, M., Stepto, N.K., Chibalin, A.V., Fryer, L.G.D., Carling, D., Krook, A., Hawley, J.A., and Zierath, J.R. 2003. Metabolic and mitogenic signal transduction in human skeletal muscle after intense cycling exercise. J Physiol 546: 327-335. 
Zammit, P.S., Golding, J.P., Nagata, Y., Hudon, V., Partridge, T.A., and Beauchamp, J.R. 2004. Muscle satellite cells adopt divergent fates: a mechanism for self-renewal? $J$ Cell Biol 166: 347-357.

Zhao, M., New, L., Kravchenko, V.V., Kato, Y., Gram, H., Di Padova, F., Olson, E.N., Ulevitch, R.J., and Han, J. 1999. Regulation of the MEF2 family of transcription factors by p38. Mol Cell Biol 19: 21-30.

Zierath, J.R., and Hawley, J.A. 2004. Skeletal muscle fiber type: influence on contractile and metabolic properties. PLoS Biol 2: e348.

Zong, H., Ren, J.M., Young, L.H., Pypaert, M., Mu, J., Birnbaum, M.J., and Shulman, G.I. 2002. AMP kinase is required for mitochondrial biogenesis in skeletal muscle in response to chronic energy deprivation. PNAS 99: 15983-15987. 